\title{
GENERALIZED RAY-SINGER CONJECTURE. I. A MANIFOLD WITH A SMOOTH BOUNDARY
}

\author{
S.M. VISHIK
}

For my parents

\begin{abstract}
This paper is devoted to a proof of a generalized Ray-Singer conjecture for a manifold with boundary (the Dirichlet and the Neumann boundary conditions are independently given on each connected component of the boundary and the transmission boundary condition is given on the interior boundary). The Ray-Singer conjecture [RS] claims that for a closed manifold the combinatorial and the analytic torsion norms on the determinant of the cohomology are equal. For a manifold with boundary the ratio between the analytic torsion and the combinatorial torsion is computed. Some new general properties of the Ray-Singer analytic torsion are found. The proof does not use any computation of eigenvalues and its asymptotic expansions or explicit expressions for the analytic torsions of any special classes of manifolds.
\end{abstract}

\section{Contents}

\section{Analytic torsion and the Ray-Singer conjecture}

1.1. Analytic and combinatorial torsions norms

1.2. Gluing formulas

1.3. Properties of analytic and combinatorial torsion norms

1.4. Generalized Ray-Singer conjecture

2. Gluing formula for analytic torsion norms. Proof of Theorem 1.134

2.1. Strategy of the proof

2.2. Continuity of the analytic torsion norms

2.3. Actions of the homomorphisms of identifications on the determinant. Proof of Lemma 2.3

2.4. Analytic torsion norm on the cone of a morphism of complexes. Proof of Lemma 2.4

2.5. Variation formula for norms of morphisms of identifications. Proof of Lemma 2.1

2.6. Variation formula for the scalar analytic torsion. Proof of Lemma 2.2 
2.7. Continuity of the truncated scalar analytic torsion. Proof of Proposition 2.1

2.8. Dependence on the phase of a cut of the spectral plane. The analytic torsions as functions of the phase of a cut. Gluing formula for the analytic torsions

\section{Zeta- and theta-functions for the Laplacians with $\nu$-transmission} interior boundary conditions

3.1. Properties of zeta- and theta-functions for $\nu$-transmission boundary conditions

3.2. Zeta-functions for the Laplacians with $\nu$-transmission interior boundary conditions. Proofs of Theorem 3.1 and of Proposition 3.1

3.3. Theta-functions for the Laplacians with $\nu$-transmission boundary conditions. Proofs of Theorem 3.2 and of Proposition 3.2

3.4. Estimates for zeta-functions and for the corresponding kernels in vertical strips in the complex plane

3.5. Appendix. Trace class operators and their traces

\section{References}

Torsion invariants for manifolds which are not simply connected were introduced by K. Reidemeister in [Re1], [Re2], where he obtained with the help of such invariants a full $P L$-classification of three-dimensional lens spaces. These invariants were generalized by W. Franz to multi-dimensional $P L$-manifolds in $[\mathrm{Fr}]$. As the result of this generalization he obtained a $P L$-classification of lens spaces of any dimension. (These torsions were the first invariants of manifolds which are not homotopy invariants.) J.H.C. Whitehead in [Wh] and G. de Rham in [dR3] introduced torsion invariants for smooth manifolds. G. de Rham in [dR3] proved that a spherical Clifford-Klein manifold (i.e., the quotient of a sphere under the fixed-point free action of a finite group of rotations) is determined up to an isometry by its fundamental group and by its Reidemeister torsions. The Whitehead torsion for a homotopy equivalence between finite cell complexes was introduced in [Wh] as a generalization of the Reidemeister torsion invariants defined in [Re1], [Fr], and [dR3]. (Its values are in the Whitehead group Wh $\left(\pi_{1}\right)$ of the fundamental group $\pi_{1}$.) The Whitehead torsion is connected with Whitehead's theory of simple homotopy types ([Wh], [dRMK], [Mi], Section 7). Some modifications of Reidemeister torsions were considered by J. Milnor in [Mi], Sections 8, 12, and by V. Turaev in [T], Section 3. The scalar Reidemeister torsion is a global invariant of a cell decomposition of a manifold and of an acyclic representation of its fundamental group. It is an invariant of the $P L$-structure of a manifold. The Reidemeister torsion for an arbitrary finite-dimensional unimodular representation of the fundamental group can be defined as a canonical norm on the 
determinant line of the cohomology of a manifold (with the coefficients in the local system defined by this representation). It is some kind of multiplicative analog of the Euler characteristic in the case of odd-dimensional manifolds. (The Euler characteristic of a closed manifold is trivial in the odd-dimensional case.) Formulas for the Reidemeister torsions of a direct product of manifolds $([\mathrm{KwS}])$ are analogous to the multiplicative property of the Euler characteristic.

The Ray-Singer analytic torsion was introduced in [RS] for a closed Riemannian manifold $\left(M, g_{M}\right)$ with an acyclic orthogonal representation of the fundamental group $\pi_{1}(M)$. It is equal to a product of the corresponding powers of the determinants of the Laplacians on differential forms $D R^{\bullet}(M)$. These determinants are regularized with the help of the zeta-functions of the Laplacians. (The scalar Reidemeister torsion also can be written by the analogous formula, where Riemannian Laplacians are replaced by the combinatorial ones.) The Ray-Singer analytic torsion is defined with the help of a Riemannian metric $g_{M}$ but it is independent of $g_{M}$ in the acyclic case. (This assertion was proved in [RS], Theorem 2.1.) So it is an invariant of a smooth structure on $M$. It has the properties analogous to the properties of the Reidemeister torsion ([RS], Sections 2, 7). The Ray-Singer conjecture $([\mathrm{RS}])$ claims that for an acyclic representation $\rho$ of the fundamental group of a closed manifold $M$ the Reidemeister torsion of $(M, \rho)$ (which is defined for any smooth triangulation of $M$ ) is equal to the Ray-Singer analytic torsion of $(M, \rho)$. This conjecture was independently proved by W. Müller in [Mü1] and by J. Cheeger in [Ch] for closed manifolds. The Ray-Singer analytic torsion can also be defined for any finite-dimensional unitary representation $\rho$ of $\pi_{1}(M)$. In this case the Ray-Singer torsion is the norm on the determinant line $\operatorname{det} H^{\bullet}(M, \rho)$. For instance, it is defined for a trivial one-dimensional representation. So the analytic torsion norm provides us with a canonical norm on the determinant line of the de Rham complex of a manifold. (The Ray-Singer formula for an arbitrary finite-dimensional unitary representation $\rho$ of $\pi_{1}(M)$ in the case, when $M$ is a smooth closed manifold, claims that the Ray-Singer norm on $\operatorname{det} H^{\bullet}(M, \rho)$ is equal to the Reidemeister norm on $\operatorname{det} H^{\bullet}(M, \rho)$.)

Let $\left(M, g_{M}\right)$ be a manifold with a smooth boundary $\partial M$ and with the Dirichlet and the Neumann boundary conditions independently given on the connected components of $\partial M$. Let $Z \subset \partial M$ be a union of the components of $\partial M$ where the Dirichlet boundary conditions are given. Let $F_{\rho}$ be a local system with a fiber $\mathbb{C}^{m}$ defined by a unitary representation $\rho: \pi_{1}(M) \rightarrow U(m)$. Then the Ray-Singer torsion norm $T_{0}\left(M, Z ; F_{\rho}\right)$ is defined on $\operatorname{det} H^{\bullet}\left(M, Z ; F_{\rho}\right)$. It is independent of $g_{M}$ (if $g_{M}$ is a direct product metric near $\partial M)$ and it depends on a flat Hermitian metric on the fibers $F_{\rho}$ (for a general $(M, Z)$ ). A flat Hermitian structure on $F_{\rho}$ defines a norm on the line $\operatorname{det}\left(F_{x}, M, Z\right):=\otimes_{k}\left(\operatorname{det} F_{x_{k}}\right)^{\chi\left(M_{k}, Z \cap \partial M_{k}\right)}$, where the product is over the full set of representatives $F_{x_{k}}$ of fibers of $F_{\rho}$ over the connected components $M_{k}$ of $M$ (with one such a fiber $F_{x_{k}}$ for each $M_{k}, x_{k} \in M_{k}$, $\operatorname{det} F_{x}:=\wedge^{\max } F_{x}$ ). The tensor product of this norm and of $T_{0}\left(M, Z ; F_{\rho}\right)$ is a modified Ray-Singer norm on $\operatorname{det} H^{\bullet}\left(M, Z ; F_{\rho}\right) \otimes$ 
$\operatorname{det}\left(F_{x}, M, Z\right)$ and it does not depend on $g_{M}$ and on a flat Hermitian metric on $F_{\rho}$ ([V1]). The Ray-Singer torsion norm for the de Rham complex of $(M, Z)$ with the coefficients in the direct sum of any finite-dimensional local system $F_{\rho}$ and of the dual one $F_{\rho}^{\vee}$ is defined in [V2]. In this case the Reidemeister torsion $\tau_{0}\left(M, Z ; F_{\rho} \oplus F_{\rho}^{\vee}\right)$ (i.e., the one for $(M, Z)$ with the coefficients in $\left.F_{\rho} \oplus F_{\rho}^{\vee}\right)$ is well-defined, because the fibers of the line bundle $\operatorname{det}\left(F_{\rho} \oplus F_{\rho}^{\vee}\right)$ have the canonical norm in accordance with the local system structure. In this case, the Ray-Singer torsion differs from the Reidemeister torsion by an explicit factor (which is computed in [V2]) but this torsion does not depend on $g_{M}$ (if $g_{M}$ is a direct product metric near $\partial M$ ). This definition of the Ray-Singer torsion norm does not use a Hermitian structure in the fibers of $F_{\rho}$. In [Mü2] another Ray-Singer torsion was introduced for the de Rham complex of a closed $\left(M, g_{M}\right)$ with the coefficients in a local system $F_{\rho}$, defined by a unimodular finite-dimensional representation $\rho$ of $\pi_{1}(M)$. This torsion is defined with the help of an arbitrary Hermitian metric $h_{\rho}$ in the fibers of $F_{\rho}$ and it depends in general on this metric. (For a non-unitary representation $\rho$ there are no Hermitian metrics on $F_{\rho}$, which are flat with respect to the canonical flat structure.) It was proved in [Mü2] that in the case of an odd-dimensional $M$ the Ray-Singer torsion, defined with the help of a Hermitian metric $h_{\rho}$, is independent of $\left(h_{\rho}, g_{M}\right)$ and is equal to the Reidemeister torsion. (The Reidemeister torsion is canonically defined for any unimodular finite-dimensional representation of $\pi_{1}$. In the case of an odddimensional closed $M$ it is independent of a flat Hermitian metric on $\operatorname{det} F_{\rho}$, since the Euler characteristic in this case is equal to zero for each connected component of $M$.) The Ray-Singer torsion, defined with the help of $h_{\rho}$, depends on $\left(h_{\rho}, g_{M}\right)$ for a general even-dimensional $M$. The definition of the Ray-Singer torsion for any finite-dimensional representation $\rho$ of $\pi_{1}(M)$ for a closed $\left(M, g_{M}\right)$ equiped with a Hermitian metric $h_{\rho}$ (on the fibers of the corresponding vector bundle) is given in [BZ1], [BZ2]. In [BZ2] the Ray-Singer metric on the determinant line, corresponding to a finite flat exact sequence $\left(F^{\bullet}, d_{F}\right)$ of finite-dimensional flat vector bundles over $M$ is computed (in terms of $g_{M}$ and of Hermitian metrics on $F^{j}$ ).

The Gaussian integral of $\exp (-(S x, x))$, where $S$ is a positive self-adjoint operator in a finite-dimensional Hilbert space $H, \operatorname{dim} H=n$, is equal to $(2 \pi)^{n / 2}(\operatorname{det} S)^{-1 / 2}$. The Ray-Singer torsion appears naturally in the computations of asymptotic expansions for analogous infinite-dimensional integrals of $\exp (-i k I(A))$, where $I(A)$ possesses an infinite-dimensional symmetry group $G$ ([Sc], [Wi1], [Wi2]). For instance, the Chern-Simons action

$$
I(A):=(4 \pi)^{-1} \int_{M} \operatorname{Tr}(A \wedge d A+2 / 3 A \wedge A \wedge A)
$$

on a trivialized principal $G$-bundle $P_{G}$ over a closed orientable three-dimensional manifold (where $G=S U_{N}$ and $\operatorname{Tr}$ is the trace in the $N$-dimensional geometrical representation of $G$, and where $A$ is a connection form) is invariant under the gauge 
transformations $A \rightarrow g A g^{-1}-d g \cdot g^{-1}=: A_{g}$ for a smooth $g: M \rightarrow G$ (where $A_{g}$ is the same connection but with respect to another trivialization of $P_{G}$, i.e., with respect to another smooth section $\left.G \rightarrow P_{G}\right)$. Stationary points of $I(A)$ are the flat connections $A_{\alpha}$ (i.e., such that the curvature $F\left(A_{\alpha}\right)$ is equal to zero). The asymptotic of an integral of $\exp (-i k I(A))$ as $k \rightarrow+\infty, k \in \mathbb{Z}_{+}$, is computed by the stationary phase method. The principal term of the contribution of a point $A_{\alpha}$ into this integral (in the case when the flat connection $A_{\alpha}$ is an isolated one) has as its absolute value the square root of the Ray-Singer torsion of $M$ with coefficients in the local system, defined by a flat connection $A_{\alpha}$, with the Lie algebra $\mathfrak{g}$ of $G$ as its fibers (see [Wi1]; [Wi2], 2.2; [BW], 2).

The Reidemeister torsion was essentially used in [Wi2], 4, for the computation of the volume of a moduli space $\mathcal{M}$ of the fundamental group representations for a closed two-dimensional surface. In this case the Reidemeister torsion is a section of $|\operatorname{det}| T^{*} \mathcal{M}$, i.e., it is a density on $\mathcal{M}$.

This paper is devoted to a proof of a generalized Ray-Singer conjecture for manifolds with a smooth boundary (and also for transmission boundary conditions given on the interior boundaries). We suppose that the local system is trivial. The proof of the Ray-Singer conjecture for non-unitary local systems and for manifolds with corners will be the subject of a subsequent paper.

Let $\left(M, g_{M}\right)$ be a Riemannian manifold with a smooth boundary $\partial M$ and let the Dirichlet and the Neumann boundary conditions be independently given on the connected components of $\partial M$. Let $g_{M}$ be a direct product metric near $\partial M$. Then the Ray-Singer torsion of $\left(M, g_{M}\right)$ is defined as a norm on the determinant line $\operatorname{det} H^{\bullet}(M, Z)$. (Here $Z$ is the union of the connected components of $\partial M$ where the Dirichlet boundary conditions are given.) This norm is independent of $g_{M}$ (for direct product metrics $g_{M}$ near $\left.\partial M\right)$. The Reidemeister torsion of $(M, Z)$ is an invariant of the $P L$-structure of $(M, Z)$ and it is a norm on the same determinant line. The torsion norms are defined in Section 1. The Ray-Singer norm differs from the Reidemeister norm on $\operatorname{det} H^{\bullet}(M, Z)$ for a general $\partial M \neq \emptyset$. Their ratio is computed in Theorem 1.4 below.

Let $\left(M, g_{M}\right)$ be obtained by gluing two Riemannian manifolds $\left(M_{j}, g_{M_{j}}\right)$ along the common component $N$ of their boundaries, $M:=M_{1} \cup_{N} M_{2}$ (where $N$ is a closed smooth manifold of codimension one in $M$ ). Let $g_{M}$ be a direct product metric near $N$. Then, as it is proved in Theorem 1.1, the Ray-Singer torsion norm $T_{0}(M, Z)$ on det $H^{\bullet}(M, Z)$ is equal to the tensor product of the Ray-Singer norms $T_{0}\left(M_{1}, Z_{1} \cup N\right) \otimes T_{0}\left(M_{2}, Z_{2} \cup N\right) \otimes T_{0}(N)\left(Z_{k}:=Z \cap \partial \bar{M}_{k}\right)$, where the line $\operatorname{det} H^{\bullet}(M, Z)$ is identified with the tensor product of the lines $\operatorname{det} H^{\bullet}\left(M_{1}, Z_{1} \cup N\right) \otimes$ $\operatorname{det} H^{\bullet}\left(M_{2}, Z_{2} \cup N\right) \otimes \operatorname{det} H^{\bullet}(N)$ by the short exact sequence of the de Rham complexes

$$
0 \rightarrow D R^{\bullet}\left(M_{1}, Z_{1} \cup N\right) \oplus D R^{\bullet}\left(M_{2}, Z_{2} \cup N\right) \rightarrow D R^{\bullet}(M, Z) \rightarrow D R^{\bullet}(N) \rightarrow 0,
$$


where $D R^{\bullet}(M, Z)$ is the relative de Rham complex of smooth forms with the zero geometrical restrictions to $Z$, the left arrow is the natural inclusion, and the right arrow is $\sqrt{2}$ times a geometrical restriction. For the Reidemeister norm this assertion is also true and the identification of the determinant lines is given by the analogous exact sequence of cochain complexes. However in this case the right arrow is the geometrical restriction of cochains (without additional factor $\sqrt{2}$ ). Let $(M, Z)$ be obtained by gluing two manifolds $\left(M_{1}, Z_{1}\right)$ and $\left(M_{2}, Z_{2}\right)$ along the common component $N$ of their boundaries, $M:=M_{1} \cup_{N} M_{2}$. Then the ratio of the square of the Ray-Singer norm and the square of the Reidemeister norm for $(M, Z)$ is equal to the product of the same ratios for $\left(M_{1}, Z_{1} \cup N\right),\left(M_{2}, Z_{2} \cup N\right)$, and for $N$ with an additional factor $2^{-\chi(N)}$. So the assertion of Theorem 1.1 claims that it is possible to calculate the Ray-Singer norm by cutting of a manifold into pieces which are manifolds with smooth boundaries. The main theorems of this paper are consequences of Theorem 1.1. This theorem provides us with the gluing formula for the Ray-Singer torsion norms. Such a gluing formula is a new one.

In the case of a manifold with a smooth boundary, the Ray-Singer torsion $T_{0}(M, Z)$ is a function not only of $(M, Z)$ but also of the phase $\theta$ of a cut of the spectral plane $\mathbb{C}$ (because the zeta-functions $\zeta_{j}(s)$ for the Laplacians $\Delta_{j}$ on $D R^{j}(M, Z)$ are defined for $\operatorname{Re} s>(\operatorname{dim} M) / 2$ as the sums $\sum \lambda^{-s}$ over the nonzero eigenvalues, and $\lambda^{-s}$ is defined as $\lambda_{(\theta)}^{-s}:=\exp \left(-s \log _{(\theta)} \lambda\right)$, where $\left.\theta-2 \pi<\operatorname{Im} \log _{(\theta)} \lambda<\theta, \theta \notin 2 \pi \mathbb{Z}\right)$. In fact, $T_{0}(M, Z ; \theta)$ (as well as $\zeta_{j}(s)$ ) depends only on $[\theta / 2 \pi]$. The zeta-function regularization of the $\operatorname{det}^{\prime}\left(\Delta_{j}\right)$ (i.e., of the product of all the nonzero eigenvalues of $\Delta_{j}$, including their multiplicities) is defined as $\exp \left(-\left.\partial_{s} \zeta_{j}(s)\right|_{s=0}\right)$. The analytic continuation of $\zeta_{j}(s)$ is regular at zero. The zeta-function $\zeta_{j}(s ; m)$ depends on $m:=[\theta / 2 \pi], \theta \notin 2 \pi \mathbb{Z}$, as follows:

$$
\begin{aligned}
\zeta_{j}(s ; m+1) & =\exp (-2 \pi i s) \zeta(s ; m), \\
\operatorname{det}^{\prime}\left(\Delta_{j} ; m+1\right) & =\exp \left(2 \pi i \zeta_{j}(0)\right) \operatorname{det}^{\prime}\left(\Delta_{j} ; m\right) .
\end{aligned}
$$

The number $\zeta_{j}(0)$ is independent of $m$, and the number $\zeta_{j}(0)+\operatorname{dim} \operatorname{Ker} \Delta_{j}$ can be interpreted as the regularized dimension of the space $D R^{j}(M)$. This regularized dimension depends not only on the space $D R^{j}(M)$ but it also depends on a positive definite self-adjoint elliptic differential operator of a positive order, which acts in $D R^{j}(M)$. This dimension is a real number but it is not an integer in the case of the Laplacians on $D R^{\bullet}(M)$ for a general closed even-dimensional $\left(M, g_{M}\right)$. Hence, $\operatorname{det}\left(\Delta_{j} ; m\right)$ depends on $m$ for such $\left(M, g_{M}\right)$. The number $\zeta_{j}(0)$ is an integer for a generalized Laplacian on a closed odd-dimensional $\left(M, g_{M}\right)$, according to [BGV], Theorem 2.30, or to [Gr], Theorem 1.6.1. It is equal to zero when $M$ is closed, $\operatorname{dim} M$ is odd, and $\operatorname{dim} \operatorname{Ker} \Delta_{j}=0$.

Even in such a simple case as for an interval $(I, \partial I)$ with the Dirichlet boundary conditions, the dependence of $T_{0}(M, Z ; m)$ on $m$ is nontrivial. The ratio of the torsion 
$T_{0}(M, Z ;[\theta / 2 \pi])$ and the Reidemeister torsion norm is computed in Theorem 2.2.

The paper is organized as follows. In Section 1 we deduce a generalization of the Ray-Singer conjecture from the gluing formula for Ray-Singer torsion norms. This formula is proved in Theorem 1.1. The proof uses $\nu$-transmission interior boundary conditions on $N$, where $\nu=(\alpha, \beta) \in \mathbb{R}^{2} \backslash(0,0)$. These interior boundary problems give us a smooth in $\nu$ family of spectral problems on $M$. Such a problem for $\nu=(1,1)$ coincides (in a spectral sense) with the spectral problem for a glued $M$. For $\nu=(0,1)$ or for $\nu=(1,0)$ it is a direct sum of spectral problems on $M_{1}$ and on $M_{2}$, i.e., the two pieces of $M$ are completely disconnected. So this family provides us with a smooth process of cutting (in a spectral sense) of $M$ in two pieces $M_{1}$ and $M_{2}$. Let $M=M_{1} \cup_{N} M_{2}$ be obtained by gluing $M_{1}$ and $M_{2}$ along the common component $N$ of their boundaries. Then the Ray-Singer norm $T_{0}\left(M_{\nu}, Z\right)$ on the determinant line $\operatorname{det} H^{\bullet}\left(M_{\nu}, Z\right)$ for the de Rham complex $D R^{\bullet}\left(M_{\nu}, Z\right)$ with $\nu$ transmission conditions on $N$ is defined. The short exact sequence for $D R^{\bullet}\left(M_{\nu}, Z\right)$, similar to (0.1), has the same the first and third terms as (0.1). The homomorphisms $r_{\nu}: D R^{\bullet}\left(M_{\nu}, Z\right) \rightarrow D R^{\bullet}(N)$ are of the form $r_{\nu}=\left(\alpha i_{1}^{*}+\beta i_{2}^{*}\right) /|\nu|$, where $i_{j}^{*} \omega_{j}$ are the geometrical restrictions to $N$ for the components $\omega_{j}$ of $\omega=\left(\omega_{1}, \omega_{2}\right) \in D R^{\bullet}\left(M_{\nu}, Z\right)$. Note that $r_{(1,1)}=\sqrt{2} i^{*}$. (This is the reason of the appearance of $\sqrt{2} i^{*}$ in the exact sequence (0.1), connected with the gluing formula.) In Lemma 1.2 we prove that the gluing property for analytic torsion norms (Theorem 1.1) is equivalent to the independence of $\nu$ of the norms on $\operatorname{det} H^{\bullet}\left(M_{1}, Z_{1} \cup N\right) \otimes \operatorname{det} H^{\bullet}\left(M_{2}, Z_{2} \cup N\right) \otimes \operatorname{det} H^{\bullet}(N)$ induced by $T_{0}\left(M_{\nu}, Z\right)$. (Here the identification of the determinant lines is defined by the short exact sequence for $D R^{\bullet}\left(M_{\nu}, Z\right)$.) The latter assertion is proved in Section 2. First we prove that the norm induced by the Ray-Singer torsion $T_{0}\left(M_{\nu}, Z\right)$ is locally independent of $\nu$ in the case when $\alpha \beta \neq 0$ (where $\nu=(\alpha, \beta)$ ). We do this in Sections 2.3, 2.5, and 2.6 with the help of explicit variation formulas for the scalar Ray-Singer torsion $T\left(M_{\nu}, Z\right.$ ) (if $\nu$ depends smoothly on a parameter). We define a family (in $\nu$ ) of homomorphisms to identify finite-dimensional subcomplexes $W_{a}^{\bullet}(\nu)$ of $D R^{\bullet}\left(M_{\nu}, Z\right)$. (The complexes $W_{a}^{\bullet}(\nu)$ are spanned by the eigenforms of the Laplacians with eigenvalues less than a fixed number $a>0$. We suppose that $a$ is not an eigenvalue of $\Delta_{j}\left(M_{\nu}, Z\right)$ for $0 \leq j \leq n$.) Then we compute the actions of these homomorphisms on the determinant lines. These identifications are not canonical; we choose some particular (quite natural) identifications for $\nu$ sufficiently close to $\nu_{0}$ such that $\alpha_{0} \beta_{0} \neq 0$.

Then it is enough to prove the continuity in $\nu \in \mathbb{R}^{2} \backslash(0,0)$ of the norm on $\operatorname{det} H^{\bullet}\left(M_{1}, Z_{1} \cup N\right) \otimes \operatorname{det} H^{\bullet}\left(M_{2}, Z_{2} \cup N\right) \operatorname{det} H^{\bullet}(N)$, which is induced by the RaySinger norm $T_{0}\left(M_{\nu}, Z\right)$. We prove in Section 2.7 that the truncated scalar analytic torsion $T\left(M_{\nu}, Z ; a\right)$, corresponding to the eigenvalues $\lambda$ of $\Delta_{j}\left(M_{\nu}, Z\right)$ which are greater than $a$, is locally continuous in $\nu$. Then we prove that the norm, induced by the analytic torsion norm $T_{0}\left(W_{a}^{\bullet}(\nu)\right)$ of a finite-dimensional complex $W_{a}^{\bullet}(\nu)$, is locally 
continuous in $\nu$. The latter assertion is proved in Sections 2.2, 2.4, and 2.7 with the use of the cone of the homomorphism $R_{\nu}^{\bullet}(a): W_{a}^{\bullet}(\nu) \rightarrow C^{\bullet}\left(X_{\nu}, Z \cap X\right)$ (where $R_{\nu}^{\bullet}(a)$ is the integration of differential forms from $W_{a}^{\bullet}$ over the simplexes of a given smooth triangulation $X$ of $M$, and $C^{\bullet}\left(X_{\nu}, Z \cap X\right)$ is the corresponding cochain complex). This homomorphism is a quasi-isomorphism for any $\nu \in \mathbb{R}^{2} \backslash(0,0)$ (Proposition 2.3). We can conclude that the analytic torsion norm on $\operatorname{det} H^{\bullet}\left(\right.$ Cone $\left.R_{\nu}(a)\right)=\mathbb{C}$ (for a fixed $\nu$ ) corresponds to an acyclic finite-dimensional complex and is defined by the derivatives at zero of the zeta-functions for self-adjoint finite-dimensional invertible operators. So these norms are locally continuous in $\nu$. (This is proved in Section 2.7.) Then the local continuity of the norm induced by $T_{0}\left(W_{a}^{\bullet}(\nu)\right)$ on $\operatorname{det} H^{\bullet}\left(M_{1}, Z_{1} \cup N\right) \otimes \operatorname{det} H^{\bullet}\left(M_{2}, Z_{2} \cup N\right) \otimes \operatorname{det} H^{\bullet}(N)$ follows from the continuity of the norm (on the same determinant line) induced by $T_{0}\left(C^{\bullet}\left(X_{\nu}, Z \cap X\right)\right)$ and from the identity:

$$
\left.T_{0}\left(W_{a}^{\bullet}\right)=T_{0}\left(C^{\bullet}\left(X_{\nu}, Z \cap X\right)\right) /\|1\|_{T_{0}(\text { Cone }}^{2} R_{\nu}(a)\right)
$$

This identity is proved in Lemma 2.4.

The use of the cone of $R_{\nu}^{\bullet}(a)$ allows us to avoid difficulties, connected with the fact that some positive eigenvalues of the Laplacians $\Delta^{\bullet}\left(M_{\nu}, Z\right)$ tend to 0 as $\nu=(\alpha, \beta)$ tends to $\nu_{0}=(1,0)$ (or to $\left.\nu_{0}=(0,1)\right)$. The dimensions of $H^{\bullet}\left(M_{\nu}, Z\right)$ essentially change when $\nu, \alpha \beta \neq 0$, is replaced by $\nu_{0}$. (Only the Euler characteristic $\chi\left(H^{\bullet}\left(M_{\nu}, Z\right)\right)$ does not change when $\nu$ is replaced by $\left.\nu_{0}.\right)$ It is impossible to find for a general $N$ the precise asymptotic expressions for the eigenvalues $\lambda$, which tend to zero as $\nu \rightarrow \nu_{0}$, and especially to find the asymptotics of the corresponding eigenforms $\omega_{\lambda}$ of $\Delta^{\bullet}\left(M_{\nu}, Z\right)$. So the continuity of the norm induced by $T_{0}\left(M_{\nu}, Z\right)$ (viewed as a function of $\nu$ ) at the point $\nu_{0}$ cannot be proved for a general $M$ (obtained by gluing two pieces $M_{1}$ and $M_{2}$ along $N$ ) with the help of separate computations of the asymptotic expressions for the scalar torsion $T\left(M_{\nu}, Z\right)$ and for the measure on $\operatorname{det} H^{\bullet}\left(M_{\nu}, Z\right)$ defined by harmonic forms. The proof of the classical Ray-Singer conjecture in [Ch] and the proof in [Mü2] (in the case of unimodular representations of $\pi_{1}(M)$ ) are based on asymptotic computations of such quantities for a manifold with boundary $M_{u}:=M \backslash S_{u}$, where $S_{u}$ is a tubular neighborhood of an embedded sphere $S^{k} \hookrightarrow M^{n}$ as the radius $u$ of the tubular neighborhood (in the normal to $S^{k}$ direction) tends to zero. (It is also supposed in [Ch] that $S_{u}$ is a direct product on $S^{k} \times D^{n-k}$ and that $\left.g_{M}\right|_{S_{u}}$ is a direct product metric on $S^{k} \times D^{n-k}$.)

To give a rigorous proof of the assertions above used in the proof of the gluing formula, it is necessary to prove a lot of analytic propositions. We do it in Sections 2.2, 2.6, 2.7, and in Section 3. The theory of $\zeta$ - and $\theta$-functions in the case of $\nu$-transmission interior boundary conditions is elaborated in Section 3. The precise estimates of the corresponding $\zeta$-functions in vertical strips are obtained in Section 3.4. These estimates allow us using the inverse Mellin transform to derive the information about the densities on $M, N$, and $\partial M$ for the asymptotic expansions as $t \rightarrow+0$ of 
$\theta$-functions from the properties of the densities for appropriate $\zeta$-functions.

\section{Analytic torsion and the Ray-Singer conjecture}

1.1. Analytic and combinatorial torsions norms. The analytic torsion norm appears in the following finite-dimensional algebraic situation. Let $\left(A^{\bullet}, d\right)$ be a finite complex of finite-dimensional Hilbert spaces. The determinant of $\left(A^{\bullet}, d\right)$ is the tensor product

$$
\otimes_{j}\left(\Lambda^{\max } A^{j}\right)^{(-1)^{j+1}}=: \operatorname{det}\left(A^{\bullet}\right)
$$

where $\Lambda^{\max } A^{j}=$ : $\operatorname{det} A^{j}$ is the top exterior power of the linear space $A^{j}$ and where $L^{-1}$ is the dual space $L^{\vee}$ for a one-dimensional vector space $L$ over $\mathbb{C}$. The natural Hilbert norm $\|\cdot\|_{\operatorname{det} A}^{2}$ is defined by the Hilbert norms on $A^{j}$.

The determinant of the cohomology $\operatorname{det} H^{\bullet}(A)$ of $\left(A^{\bullet}, d\right)$ is also defined and there is a natural norm on it (since $H^{j}(A)$ is the subquotient of $A^{j}$ ). The differential $d$ provides us with the identification

$$
f(d): \operatorname{det}\left(A^{\bullet}\right) \simeq \operatorname{det} H^{\bullet}(A) .
$$

However in the general case this identification is not an isometry of the norms $\|\cdot\|_{\operatorname{det} A}^{2}$ and $\|\cdot\|_{\operatorname{det} H \bullet(A)}^{2}$. For $f(d)$ to be an isometry it is necessary to multiply $\|\cdot\|_{\operatorname{det} H \bullet(A)}^{2}$ by the scalar analytic torsion of a complex $\left(A^{\bullet}, d\right)$, which is defined as

$$
T\left(A^{\bullet}, d\right)=\exp \left(\left.\Sigma(-1)^{j} j \partial_{s} \zeta_{j}(s)\right|_{s=0}\right) .
$$

Here $\zeta_{j}(s)=\sum^{\prime} \lambda^{-s}$ is the $\operatorname{sum}^{1}$ over all the nonzero eigenvalues $\lambda \neq 0$ (including their multiplicities) of the nonnegative (i.e., if $\lambda \neq 0$ then $\lambda>0$ ) self-adjoint operator $\left(d^{*} d+d d^{*}\right) \mid A^{j}$. The derivative $\left.\partial_{s} \zeta_{j}(s)\right|_{s=0}$ is equal to $-\log \operatorname{det}^{\prime}\left(\left.\left(d^{*} d+d d^{*}\right)\right|_{A^{j}}\right)$ (i.e., it is equal to the sum of $(-\log \lambda) \in \mathbb{R}$ over all the nonzero eigenvalues $\lambda)$.

It is enough to prove the assertion (1.1) in the case of a two-terms complex $d: F_{0} \vec{\sim}$ $F_{1}$, where $\operatorname{dim} F_{j}=1, e_{j} \in F_{j}, d e_{0}=\mu e_{1}, \mu \neq 0$, and where $\left\|e_{0}\right\|^{2}=1=\left\|e_{1}\right\|^{2}$. In this case the element $e_{1} \otimes e_{0}^{-1} \in \operatorname{det}\left(F^{\bullet}\right)$ is of the unit norm and the square of the norm of the corresponding element $\mu^{-1} \in \mathbb{C}$ from $\mathbb{C}=\operatorname{det} 0=\operatorname{det} H^{\bullet}(F)$ is equal to $\left|\mu^{-2}\right|^{2}$. If the norm $\left|\mu^{-1}\right|^{2}$ is multiplied by the scalar analytic torsion for $F^{\bullet}$, namely by $\exp \left(\log \operatorname{det}\left(d^{*} d\right)\right)=\exp \left(\log \operatorname{det}\left(d d^{*}\right)\right)=|\mu|^{2}$ then the isomorphism between $\operatorname{det}\left(F^{\bullet}\right)$ and $\mathbb{C}=\operatorname{det} 0$ (defined by $d$ ) becomes an isometry.

This finite-dimensional definition make sense also for the infinite-dimensional de Rham complex of a closed smooth manifold. In this case the analytic torsion is the norm on the determinant of the cohomology of this manifold. Let $\left(D R^{\bullet}(M), d\right)$ be the de Rham complex of smooth differential forms (with the values in $\mathbb{C}$ ) on a closed manifold $M$. The scalar analytic torsion for a closed Riemannian manifold $\left(M, g_{M}\right)$ is defined by the same formula $(1.1)$, where $d^{*}=\delta$ (relative to $g_{M}$ ) and

\footnotetext{
${ }^{1}$ The function $\lambda^{-s}$ is defined as $\exp (-s \log \lambda)$ where $\log \lambda \in \mathbb{R}$ for $\lambda \in \mathbb{R}_{+}$.
} 
$\left(d^{*} d+d d^{*}\right) \mid D R^{j}(M)$ is the Laplace-Beltrami operator $\Delta_{j}$. In this case the series, which defines $\zeta_{j}(s)$, converges for $\operatorname{Re} s>(\operatorname{dim} M) / 2$. The analytic function $\zeta_{j}(s)$ can be analytically (meromorphically) continued to the whole complex plane. It is known that $\zeta_{j}(s)$ has simple poles and that it is regular at zero ([Se2]).

The cohomology $H^{\bullet}(D R(M))$ are canonically identified (by the integration of the forms over the simplexes) with the cohomology $H^{\bullet}(M)$ of $M$. This follows from the de Rham theorem. The Hodge theorem claims that each element of $H^{j}(D R(M))$ has one and only one representative in the space of harmonic forms $\operatorname{Ker} \Delta_{j}$. The natural norm on Ker $\Delta_{j}$ (defined by the Riemannian metric $g_{M}$ ) provides us with the norm $\|\cdot\|_{\operatorname{det} H \bullet(M)}^{2}$ on $\operatorname{det} H^{\bullet}(M)$. For an odd-dimensional $M$ this norm depends on $g_{M}$.

Definition. The analytic torsion norm $T_{0}(M)$ on $\operatorname{det} H^{\bullet}(M)$ is the norm

$$
T_{0}(M):=\|\cdot\|_{\operatorname{det} H \bullet(M)}^{2} \cdot \exp \left(\left.\Sigma(-1)^{j} j \partial_{s} \zeta_{j}(s)\right|_{s=0}\right) .
$$

The main property of this norm is its independence of a Riemannian metric $g_{M}$. So it is an invariant of a smooth structure on $M$. Let us suppose that $g_{M}=g_{M}(\gamma)$ depends smoothly on a parameter $\gamma \in \mathbb{R}^{1}$. Then the variation formulas in [RS], Theorems 2.1, 7.3 (or in [Ch], Theorem 3.10, (3.22)), claim that

$$
\left.\partial_{\gamma} \sum_{j}(-1)^{j} j \partial_{s} \zeta_{j, \gamma}(s)\right|_{s=0}=\sum_{j}(-1)^{j}\left(-\operatorname{Tr}\left(\exp \left(-t \Delta_{j, \gamma}\right) \alpha\right)^{0}+\operatorname{Tr}\left(\mathcal{H}_{j, \gamma} \alpha\right)\right) \text {. }
$$

Here $\mathcal{H}_{j, \gamma}$ is the kernel of the orthogonal projection operator from $D R^{j}(M)$ onto $\operatorname{Ker} \Delta_{j}\left(M, g_{M}(\gamma)\right), \alpha:=*_{\gamma}^{-1} \partial_{\gamma}\left(*_{\gamma}\right)\left(*_{\gamma}\right.$ corresponds to $\left.g_{M}(\gamma)\right)$ and $\operatorname{Tr}\left(\exp \left(-t \Delta_{j, \gamma}\right) \alpha\right)^{0}$ is the constant coefficient in the asymptotic expansion as $t \rightarrow+0(n:=\operatorname{dim} M)$ :

$$
\operatorname{Tr}\left(\exp \left(-t \Delta_{j, \gamma}\right) \alpha\right)=\sum_{k=0}^{l} m_{j, k} t^{-n / 2+k}+o\left(t^{l}\right) .
$$

The existence of the asymptotic expansion (1.4) follows from [Gr], Theorem 1.6.1, or from $[\mathrm{BGV}]$, Theorem 2.30. For a family of norms $\|\cdot\|^{2}(\gamma)$ on $\operatorname{det} H^{\bullet}(M)$ defined by the harmonic forms $\operatorname{Ker}\left(\Delta_{j}\left(M, g_{M}(\gamma)\right)\right)$ the following equality holds for any fixed $\mu \in \operatorname{det} H^{\bullet}(M), \mu \neq 0([\mathrm{RS}]$, Section 7$)$ :

$$
\partial_{\gamma} \log \|\mu\|_{\operatorname{det} H \bullet(M)}^{2}(\gamma)=-\sum(-1)^{j} \operatorname{Tr}\left(\mathcal{H}_{j, \gamma} \alpha\right) .
$$

Hence, (1.3) involves the equality

$$
\partial_{\gamma} \log T_{0}\left(M, g_{M}\right)=\sum(-1)^{j+1} m_{j, n / 2}
$$

Since $k$ in (1.4) are integers, we see that the right side of (1.5) is zero for odd $n$. For even $n, n=2 l$, the right side of (1.5) is also equal to zero, since $m_{j, l}=-m_{2 l-j, l}$. 
This fact follows from the equalities

$$
\begin{gathered}
\partial_{\gamma}\left(*_{\gamma}^{-1} *_{\gamma}\right)=0, \quad \alpha=-* \alpha *^{-1}, \\
\operatorname{Tr}\left(\exp \left(-t \Delta_{j}\right) \alpha\right)=\operatorname{Tr}\left(\left(* \exp \left(-t \Delta_{j}\right) *^{-1}\right)(-\alpha)\right)=-\operatorname{Tr}\left(\exp \left(-t \Delta_{n-j}\right) \alpha\right),
\end{gathered}
$$

(since they involve the equalities $m_{j, k}=-m_{n-j, k}$, where $n$ is even and $k \in \mathbb{Z}_{+} \cup 0$ ).

The analytic torsion norm can be interpreted (in an intuitional sense) as the norm, corresponding to an element $v \in \operatorname{det} D R^{\bullet}(M)$ ( $v$ is defined up to a multiplicative constant $c \in \mathbb{C},|c|=1$, and its "torsion norm" is equal to one). The space $\operatorname{det} D R^{\bullet}(M)$ and $L_{2}$-norm on it are not defined but the space $\operatorname{det} H^{\bullet}(M)$ and the analytic torsion norm $T_{0}(M)$ on it are rigorously defined. For a finite-dimensional complex the analytic torsion norm on the determinant of its cohomology corresponds to the norm on the determinant of the complex defined by the Hilbert structures on the terms of this complex. The analytic torsion norm is (in some sense) a multiplicative Euler characteristic useful for odd-dimensional manifolds.

The same definition of $T_{0}(M)$ make sense also in the case when $M$ is a compact Riemannian manifold with a smooth boundary $\partial M=\cup N_{i}$ and with the Dirichlet or the Neumann boundary conditions given independently on each connected component $N_{i}$ of $\partial M$. Let the metric $g_{M}$ be a direct product metric near $\partial M$. Then $T_{0}(M)$ is independent of $g_{M}$ as in the case of a closed manifold (this is proved below).

Let $X$ be a smooth triangulation of $M$ and let $\left(C^{\bullet}(X), d_{c}\right)$ be a cochain complex of $X$ ( with complex coefficients). Then each $C^{j}(X)$ has the Hilbert structure defined by the orthonormal basis of basic cochains $\left\{\delta_{e}\right\}$, where $\delta_{e}\left(e_{1}\right)$ is 1 for $e_{1}=e$ and 0 for $e_{1} \neq e$. Hence the scalar torsion $T\left(C^{\bullet}(X), d_{c}\right)$ is also defined.

The combinatorial torsion $\tau_{0}(X)$ is defined as the following norm on the determinant of the cohomology $H^{\bullet}\left(C(X), d_{c}\right)=H^{\bullet}(M)$ :

$$
\tau_{0}(X):=\|\cdot\|_{\operatorname{det} H \bullet(C(X))}^{2} \cdot T\left(C^{\bullet}(X), d_{c}\right)
$$

(where $H^{j}(C(X))$ is the subquotient of $C^{j}(X)$ and so it has the natural Hilbert structure induced from $\left.C^{j}(X)\right)$. The norm (1.6) is invariant under any regular subdivisions of $X$. So this norm is an invariant of the combinatorial structure of $M$ (which is completely defined by a smooth structure on $M$ ). This norm corresponds to the Hilbert norm on $\operatorname{det} C^{\bullet}(X)$, defined by the basic cochains.

Let $M$ be a manifold with a smooth boundary $\partial M=\cup N_{i}$, where $N_{i}$ are the connected components of $\partial M$. Let $Z$ be the union of $N_{i}$ where the Dirichlet boundary conditions are given. Set $V:=X \cap Z$. Then (1.6) (where $H^{\bullet}(C(X))$ and $T\left(C^{\bullet}(X), d_{c}\right)$ are replaced by $H^{\bullet}(C(X, V))$ and by $\left.T\left(C^{\bullet}(X, V), d_{c}\right)\right)$ provides us with the definition of the norm $\tau_{0}(X, V)$. This norm is an invariant of the combinatorial structure on $(M, Z)([\mathrm{Mi}]$, Sections $7,8,9)$. 
1.2. Gluing formulas. The Ray-Singer conjecture claims that for a closed smooth manifold $M$ the norms $\tau_{0}(M)$ and $T_{0}(M)$ on the same one-dimensional space $\operatorname{det} H^{\bullet}(M)$ are equal $^{2}$

$$
\tau_{0}(M)=T_{0}(M)
$$

How to prove such a formula in a natural way? It is necessary to find a general property of the analytic torsion which involves the equality (1.7). Such a property can be formulated as follows. Let $(M, \partial M)$ be a Riemannian manifold with a smooth boundary and with the Dirichlet or the Newmann boundary conditions given independently on the connected components of $\partial M$. Let a closed codimension one submanifold $N$ of $M, N \cap \partial M=\emptyset$, divides $M$ in two pieces $M_{1}$ and $M_{2}$ (glued along $N), M=M_{1} \cup_{N} M_{2}$, and let a metric $g_{M}$ be a direct product metric near $N$ and near $\partial M$. Let $T_{0}\left(M_{k}, N\right)$ be the analytic torsion norm for $M_{k}$ (with the Dirichlet boundary conditions on $N$ ), and let the boundary conditions on the connected components of $\partial M$ belonging to $\partial M_{k}$ be the same as for $T_{0}(M)$. The following assertion central in this paper.

Theorem 1.1 (Gluing property). The analytic torsion norm $T_{0}(M, Z)$ is the tensor product of the analytic torsion norms for $\left(M_{1}, Z_{1} \cup N\right),\left(M_{2}, Z_{2} N\right)$, and for $N$

$$
\varphi_{a n} T_{0}(M, Z)=T_{0}\left(M_{1}, Z_{1} \cup N\right) \otimes T_{0}\left(M_{2}, Z_{2} \cup N\right) \otimes T_{0}(N),
$$

where $Z_{k}:=Z \cap \partial \bar{M}_{k}$.

The identification $\varphi_{\text {an }}$ (in (1.8)) of $\operatorname{det} H^{\bullet}(M, Z)$ with the tensor product of the three one-dimensional spaces:

$$
\begin{array}{r}
\varphi_{a n}: \operatorname{det} H^{\bullet}(M, Z) \rightarrow \operatorname{det} H^{\bullet}\left(M_{1}, Z_{1} \cup N\right) \otimes \operatorname{det} H^{\bullet}\left(M_{2}, Z_{2} \cup N\right) \otimes \operatorname{det} H^{\bullet}(N)= \\
=: \operatorname{Det}(M, N, Z)
\end{array}
$$

is defined by the long cohomology exact sequence corresponding to the following short exact sequence of the de Rham complexes:

$$
0 \rightarrow D R^{\bullet}\left(M_{1}, Z_{1} \cup N\right) \oplus D R^{\bullet}\left(M_{2}, Z_{2} \cup N\right) \rightarrow D R^{\bullet}\left(M_{1,1}, Z\right) \stackrel{r}{\rightarrow} D R^{\bullet}(N) \rightarrow 0 .
$$

The relative de Rham complex $\left(D R^{\bullet}\left(M_{k}, Z_{k} \cup N\right), d\right.$ ) (where $d$ is the exterior derivative of differential forms) consists of the smooth forms $\omega$ on $\bar{M}_{k}$, having the zero geometrical restriction to $N: i_{k}^{*} \omega=0$ (where $i_{k}: N \subset \partial M_{k} \hookrightarrow M_{k}$ ) and also having the zero restrictions to the components of $\partial M \cap M_{k}$, where the Dirichlet boundary conditions are given (i.e., to $\left.Z_{k}\right)$. The complex $\left(D R\left(M_{1,1}\right), d\right)$ consists of the pairs $\left(\omega_{1}, \omega_{2}\right)$ of smooth differential forms $\omega_{k} \in D R^{\bullet}\left(M_{k}, Z_{k}\right)$ (i.e., $\omega_{k}$ have the

\footnotetext{
${ }^{2}$ The cohomology $H^{\bullet}(D R(M))$ and $H^{\bullet}(C(X))$ are identified (according to the de Rham theorem) by the homomorphism of the integration of forms from $D R^{\bullet}(M)$ over the simplexes of a smooth triangulation $X$ of $M$.
} 
zero geometrical restrictions to the corresponding components of $\partial M \cap \bar{M}_{k}$ ), which have the same geometrical restrictions to $N$ :

$$
i_{1}^{*} \omega_{1}=i_{2}^{*} \omega_{2}
$$

The differential $d\left(\omega_{1}, \omega_{2}\right)$ in $D R^{\bullet}\left(M_{1,1}\right)$ is defined as $\left(d \omega_{1}, d \omega_{2}\right)$. The left arrow in (1.10) is the natural inclusion of $\oplus_{k} D R^{\bullet}\left(M_{k}, Z_{k} \cup N\right)$ into $D R^{\bullet}\left(M_{1,1}, Z\right)$. The right arrow $r$ in (1.10) is not a usual geometrical restriction but is the one multiplied by $\sqrt{2}$ :

$$
r\left(\omega_{1}, \omega_{2}\right)=\sqrt{2} i_{k}^{*} \omega_{k} \in D R^{\bullet}(N) .
$$

To define $\varphi_{\text {an }}$ it is necessary to introduce a natural identification of $H^{\bullet}(D R(M, Z))$ with $H^{\bullet}\left(D R\left(M_{1,1}, Z\right)\right)$. (The short exact sequence (1.10) provides us with the identification

$$
\varphi_{a n}: \operatorname{det} H^{\bullet}\left(D R\left(M_{1,1}, Z\right)\right) \underset{\sim}{\operatorname{Det}}(M, N, Z)
$$

but not with the identification of $\operatorname{det} H^{\bullet}(D R(M, Z))$ with $\operatorname{Det}(M, N, Z)$.) We show in Proposition 1.1 (for any given metric $g_{M}$ ) that not only all the eigenvalues with their multiplicities but also all the eigenforms of the natural Laplacian $\Delta_{1,1}$ on $D R^{\bullet}\left(M_{1,1}, Z\right)$ are the same as for the Laplacian on $D R^{\bullet}(M, Z)$. Thus, the operator $\Delta_{1,1}\left(g_{M}\right)$ in a very strict spectral sense is the same as $\Delta\left(g_{M}\right)$.

The homotopy operator between the identity operator on $D R^{\bullet}\left(M_{1,1}, Z\right)$ and the projection operator from $D R^{\bullet}\left(M_{1,1}, Z\right)$ onto $\operatorname{Ker}^{\bullet} \Delta_{1,1}=\operatorname{Ker}^{\bullet} \Delta$ is obtained with the help of the Green function $G_{1,1}$ for the operator $\Delta_{1,1}$ (Lemma 1.1). This homotopy operator provides us with the canonical identification of $H^{\bullet}\left(D R\left(M_{1,1}, Z\right)\right)$ with $\operatorname{Ker} \Delta_{1,1}^{\bullet}$. So it defines the identification of $H^{\bullet}\left(D R\left(M_{1,1}, Z\right)\right)$ with $\operatorname{Ker} \Delta^{\bullet}=$ $H^{\bullet}(D R(M, Z))$ (since $\operatorname{Ker} \Delta^{\bullet}$ is canonically identified with $\operatorname{Ker} \Delta_{1,1}^{\bullet}$ ).

To prove Theorem 1.1 we introduce a family of interior boundary conditions on $N$ and show that the induced norm $\varphi_{a n}^{\nu} T_{0}\left(M_{\nu}, Z\right)$ on $\operatorname{Det}(M, N, Z)$ is independent of $\nu$ (where $\nu=(\alpha, \beta) \in \mathbb{R}^{2} \backslash(0,0)$ are the parameters of interior boundary conditions on $N)$. Namely

$$
\varphi_{\nu}^{a n} T_{0}\left(M_{\nu}, Z\right)=c_{0} T_{0}\left(M_{1}, Z_{1} \cup N\right) \otimes T_{0}\left(M_{2}, Z_{2} \cup N\right) \otimes T_{0}(N)
$$

with some positive $c_{0}$ which may depend on $\left(M, g_{M}, \partial M\right)$ and on the boundary conditions on $\partial M$ but does not depend on the parameters $(\alpha, \beta)=\nu$. Suppose that the formula (1.12) holds for any gluing two pieces $M_{1}$ and $M_{2}$ along a closed $N$, $M=M_{1} \cup_{N} M_{2}$, where the factor $c_{0}$ is independent of $\nu$. Then it is easy to conclude that $c_{0}=1$ (Lemma 1.2). In (1.12) $T_{0}\left(M_{\nu}, Z\right)$ is the analytic torsion norm for the de Rham complex $\left(D R^{\bullet}\left(M_{\nu}, Z\right), d\right)$. This complex consists of the pairs of smooth forms $\left(\omega_{1}, \omega_{2}\right)$ such that $\omega_{k} \in D R \bullet\left(\bar{M}_{k}, Z_{k}\right)$ has the zero geometrical restrictions to $Z_{k}:=Z \cap \partial M_{k}{ }^{3}$ and that the following transmission condition holds for the

${ }^{3} Z$ is the union of the components of $\partial M$ where the Dirichlet boundary conditions are given. 
geometrical restrictions $i_{k}^{*} \omega_{k}$ of $\omega_{k}$ to $N$

$$
\alpha i_{1}^{*} \omega_{1}=\beta i_{2}^{*} \omega_{2}
$$

The analytic torsion norm $T_{0}\left(M_{\nu}, Z\right)$ is defined for an arbitrary $\nu=(\alpha, \beta) \in$ $\mathbb{R}^{2} \backslash(0,0)$. There is a canonical identification of $H^{j}\left(D R^{\bullet}\left(M_{\nu}, Z\right)\right)$ with the space of the corresponding harmonic forms $\operatorname{Ker}\left(\Delta_{\nu} \mid D R^{j}\left(M_{\nu}, Z\right)\right.$ ) (Lemma 1.1). This identification (similarly to the case of $D R^{\bullet}\left(M_{1,1}, Z\right)$ ) is obtained by the homotopy operator, which is defined using the Green function for the Laplacian $\Delta_{\nu}$. (This Laplacian is an elliptic self-adjoint operator by Theorem 3.1.) The boundary conditions for $\Delta_{\nu}$ on $N$ and $\partial M$ are elliptic (and differential). The Green function $G_{\nu}$ for $\Delta_{\nu}$ exists (and depends smoothly on $\nu \neq(0,0)$ ) according to Theorem 3.1 and to Proposition 3.1. This identification provides us with the natural norms on $H^{j}\left(D R^{\bullet}\left(M_{\nu}, Z\right)\right)=: H^{j}\left(M_{\nu}, Z\right)$ and on $\operatorname{det} H^{\bullet}\left(M_{\nu}, Z\right)$. The scalar analytic torsion $T\left(M_{\nu}, Z\right)$ is defined by $\zeta_{\nu, j}(s):=\sum^{\prime} \lambda_{i}^{-s}$ for $\operatorname{Re} s>(\operatorname{dim} M) / 2$ (where the sum is over all the nonzero eigenvalues $\lambda_{i}$ of the Laplacian $\Delta_{\nu, j}:=\Delta_{\nu} \mid D R^{j}\left(M_{\nu}, Z\right)$ with their multiplicities). These functions $\zeta_{\nu, j}$ can be continued to meromorfic functions on the whole complex plane with simple poles and regular at zero. (This statement is proved in Theorem 3.1 and in Proposition 3.1 below.)

The analytic torsion norm on $\operatorname{det} H^{\bullet}\left(M_{\nu}, Z\right)$ is the norm

$$
T_{0}\left(M_{\nu}, Z\right)=\|\cdot\|_{\operatorname{det} H \bullet\left(M_{\nu}, Z\right)}^{2} \exp \left(\left.\sum(-1)^{j} j \partial_{s} \zeta_{\nu, j}(s)\right|_{s=0}\right) .
$$

The identification $\varphi_{\nu}^{a n}$ in $(1.12)$ is defined by the short exact sequence of the de Rham complexes (where $Z_{k}:=Z \cap \partial \bar{M}_{k}$ ):

$$
0 \rightarrow D R^{\bullet}\left(M_{1}, Z_{1} \cup N\right) \oplus D R^{\bullet}\left(M_{2}, Z_{2} \cup N\right) \rightarrow D R^{\bullet}\left(M_{\alpha, \beta}, Z\right) \stackrel{r_{\alpha, \beta}}{\longrightarrow} D R^{\bullet}(N) \rightarrow 0
$$

The left arrow in (1.14) is the natural inclusion and the right arrow $r_{\alpha, \beta}$ is

$$
r_{\alpha, \beta}\left(\omega_{1}, \omega_{2}\right):=\left(\alpha^{2}+\beta^{2}\right)^{-1 / 2}\left(\beta i_{1}^{*} \omega_{1}+\alpha i_{2}^{*} \omega_{2}\right) .
$$

For $(\alpha, \beta)=(1,1)$ we have $r_{1,1}=\sqrt{2} i_{k}^{*} \omega_{k}$. This corresponds to (1.11). Hence, $\varphi_{a n}$ is equal to $\varphi_{\alpha, \beta}^{a n}$ for $(\alpha, \beta)=(1,1)$.

The complex $D R^{\bullet}\left(M_{\nu}, Z\right)$ for the values $(0,1)$ and $(1,0)$ of $\nu$ is the direct sum of the de Rham complexes of all the smooth forms (with the zero geometrical restriction to $Z_{k}$ ) on one of the manifolds $M_{k}$ and of all the smooth forms with the zero geometrical restriction to $Z_{j} \cup N$ on another piece $M_{j}$ of the manifold $M$. Thus, the two pieces of $M$ are completely disconnected with respect to $D R^{\bullet}\left(M_{\nu}, Z\right)$ for these special values of $\nu$. The family of spectral problems on $D R^{\bullet}\left(M_{\nu}, Z\right)$ for $\nu \in \mathbb{R}^{2} \backslash(0,0)$ provides us with a smooth deformation between a spectral problem on $M$ (without any interior boundary conditions) and the direct sum of spectral problems on $\left(M_{1}, Z_{1}\right)$ and on 
$\left(M_{2}, Z_{2} \cup N\right)$. So this family of interior boundary problems is (in a spectral sense) a kind of a smooth cutting of $M$ in two disconnected pieces.

Let $\left(M_{1}, N\right)$ be a compact smooth Riemannian manifold $\left(M_{1}, g_{M_{1}}\right)$ with a smooth boundary $\partial M_{1}$ and let $N$ be a union of some connected components of $\partial M_{1}$. Let a metric $g_{M_{1}}$ be a direct product metric near the boundary. Then (as it follows from the equality (1.8)) the analytic torsion norm $T_{0}\left(M_{1}, N\right)$ on $\operatorname{det} H^{\bullet}\left(D R\left(M_{1}, N\right)\right)$ does not depend on $g_{M_{1}}$. To prove this it is enough to take as $\left(M, g_{M}\right)$ a closed manifold $M=M_{1} \cup_{N} M_{1}$ with a mirror symmetric (with respect to $N$ ) Riemannian metric $g_{M}$ which coincides with $g_{M_{1}}$ on each piece $M_{1}$ of $M\left(g_{M_{1}}\right.$ is a direct product metric near $N$ and so $g_{M}$ is smooth on $\left.M\right)$. Since the torsions $T_{0}(M)$ and $T_{0}(N)$ are independent of $g_{M_{1}}$ and of $g_{N}=\left.g_{M_{1}}\right|_{T} N$ we see that $T_{0}\left(M_{1}, N\right)$ does not depend on $g_{M_{1}}$.

It follows from the equality (1.12) with $c_{0}=1$ that $T_{0}\left(M_{\nu}, Z\right)$ does not depend on $g_{M}$. Indeed, $T_{0}\left(M_{j}, Z_{j} \cup N\right)$ and $T_{0}(N)$ are independent of $g_{M}$, and the identification $\varphi_{\nu}^{a n}$ is also independent of $g_{M}$. (Here $M$ is a manifold with a smooth boundary $\partial M$, $N \cap \partial M=\emptyset$, the Dirichlet boundary conditions are given on a union $Z$ of some components of $\partial M$, the Neumann boundary conditions are given on $\partial M \backslash Z$, and $g_{M}$ is a direct product metric near $\partial M$ and near $N, Z_{k}:=Z \cap \partial \bar{M}_{k}$.)

Since $D R^{\bullet}\left(M_{0,1}, Z\right)$ is the direct sum $D R^{\bullet}\left(M_{1}, Z_{1}\right) \oplus D R^{\bullet}\left(M_{2}, Z_{2} \cup N\right)$ of the de Rham complexes $\left(Z_{k}:=Z \cap \partial \bar{M}_{k}\right)$, we see that the analytic torsion norm $T_{0}\left(M_{0,1}\right)$ is canonically equal to the tensor product of norms:

$$
T_{0}\left(M_{0,1}, Z\right)=T_{0}\left(M_{1}, Z_{1}\right) \otimes T_{0}\left(M_{2}, Z_{2} \cup N\right) .
$$

The determinant line in (1.16) is the tensor product

$$
\operatorname{det} H^{\bullet}\left(M_{0,1}, Z\right)=\operatorname{det} H^{\bullet}\left(M_{1}, Z_{1}\right) \otimes \operatorname{det} H^{\bullet}\left(M_{2}, Z_{2} \cup N\right)
$$

(where $H^{\bullet}\left(M_{1}, Z_{1}\right)$ and $H^{\bullet}\left(M_{2}, Z_{2} \cup N\right)$ are the relative cohomology).

The formula (1.8) claims for $\nu=(0,1)$ that

$$
\varphi_{0,1}^{a n} T_{0}\left(M_{0,1}, Z\right)=T_{0}\left(M_{1}, Z_{1} \cup N\right) \otimes T_{0}\left(M_{2}, Z_{2} \cup N\right) \otimes T_{0}(N) .
$$

It follows from the definition of the exact sequence (1.14) that $\varphi_{0,1}^{a n}$ is the identity on the component $\operatorname{det} H^{\bullet}\left(M_{2}, Z_{2} \cup N\right)$ of $\operatorname{det} H^{\bullet}\left(M_{0,1}, Z\right)$. The following theorem is an immediate consequence of (1.16) and (1.17). Let $N$ be a union of some connected components of $\partial M_{1}$, let $M_{1}$ be a compact Riemannian manifold with a smooth boundary $\partial M_{1}$ and let $Z_{1}$ be a union of some connected components of $\partial M_{1}$ not belonging to $N$. Suppose that the metric $g_{M_{1}}$ is a direct product metric near $\partial M_{1}$.

Theorem 1.2 (Gluing of boundary components). The equality holds

$$
\varphi_{a n} T_{0}\left(M_{1}, Z_{1}\right)=T_{0}\left(M_{1}, Z_{1} \cup N\right) \otimes T_{0}(N) .
$$


The identification of the determinant lines in (1.18)

$$
\varphi_{a n}: \operatorname{det} H^{\bullet}\left(M_{1}, Z_{1}\right) \underset{\sim \operatorname{det}}{\sim} H^{\bullet}\left(M_{1}, Z_{1} \cup N\right) \otimes \operatorname{det} H^{\bullet}(N)
$$

is defined by the short exact sequence of the de Rham complexes:

$$
0 \rightarrow D R^{\bullet}\left(M_{1}, Z_{1} \cup N\right) \rightarrow D R^{\bullet}\left(M_{1}, Z_{1}\right) \rightarrow D R^{\bullet}(N) \rightarrow 0
$$

where the left arrow is the natural inclusion, and the right arrow is the geometrical restriction.

Example 1.1. Formula (1.18) contains the Lerch formula ([WW], 13.21, 12.32) for the derivative at zero of the zeta-function of Riemann $\zeta(s)$ (defined for $\operatorname{Re} s>1$ as $\left.\sum_{n \geq 1} n^{-s}\right)$ :

$$
\left.\partial_{s} \zeta(s)\right|_{s=0}=-2^{-1} \log 2 \pi .
$$

Indeed, let $M$ be an interval $(0, b] \subset \mathbb{R}$ with the Dirichlet boundary conditions at 0 and the Neumann conditions at $b$. Set $N$ be a point $b$. Then the formula (1.18) claims in this case that

$$
T_{0}((0, b])=T_{0}((0, b)) \otimes T_{0}(b) .
$$

The cohomology $H^{\bullet}((0, b])=H^{\bullet}([0, b], 0)$ are trivial. The scalar analytic torsion $T((0, b])$ is equal to $\exp \left(-\left.\partial_{s} \zeta_{1}(s ; M)\right|_{s=0}\right)$, where $\zeta_{1}(s ; M)$ is the zeta-function for the Laplacian on $D R^{1}((0, b])$. This zeta-function for $\operatorname{Re} s>1 / 2$ is defined by the series

$$
\zeta_{1}(s ; M)=\sum_{n \geq 0}\left(((\pi / 2 b)(2 n+1))^{2}\right)^{-s} .
$$

So $\zeta_{1}(s ; M)=(\pi / 2 b)^{-2 s}\left(1-2^{-2 s}\right) \zeta(2 s)$ for $\operatorname{Re} s>1 / 2$, where $\zeta(s)$ is the zetafunction of Riemann. Hence, the latter equality between the analytic continuations of $\zeta_{1}(s ; M)$ and of $\zeta(2 s)$ holds for all $s \in \mathbb{C}$, and $\left.\partial_{s} \zeta_{1}(s)\right|_{s=0}=2 \zeta(0) \log 2$.

The determinant line $\operatorname{det} H^{\bullet}(M)$ on the left in (1.21) is canonically isomorphic to $\mathbb{C}$, and the $T_{0}(M)$-norm of the element $1 \in \mathbb{C}$ is equal to

$$
\|1\|_{T_{0}(M)}^{2}=\exp \left(-\zeta_{1}^{\prime}(0 ; M)\right)=\exp (-2 \zeta(0) \log 2)=2 .
$$

(Note, that the function $2 \zeta(2 s)$ is the zeta-function for the Laplacian $\Delta=\left(-\partial^{2} / \partial x^{2}\right)$ on functions on the circle of the length $2 \pi$. As the circle is odd-dimensional, then the value of $2 \zeta(2 s)$ at zero is equal to $-\operatorname{dim} \operatorname{Ker} \Delta=-1$. Hence, $2 \zeta(0)=-1$.)

The scalar analytic torsion $T((0, b))$ is equal to $\exp \left(-\partial_{s} \zeta_{1}(s ; M, N)\right)$, where $\zeta_{1}(s ; M, N)$ for $\operatorname{Re} s>1 / 2$ is defined by the series

$$
\zeta_{1}(s ; M, N)=\sum_{n \geq 1}\left(((\pi / b) n)^{2}\right)^{-s}=(\pi / b)^{-2 s} \zeta(2 s) .
$$


Hence, this equality holds for all $s \in \mathbb{C}$, and the scalar analytic torsion is equal to

$$
T((0, b))=\exp \left(-2 \zeta^{\prime}(0)+2 \zeta(0) \log (\pi / b)\right)=\exp \left(-\log (\pi / b)-2 \zeta^{\prime}(0)\right) .
$$

The identification of the determinant lines on the right and on the left in (1.21) is defined by the cohomology exact sequence

$$
0 \rightarrow H^{0}(b) \rightarrow H^{1}((0, b)) \rightarrow 0 .
$$

The element $1 \in H^{0}(b)$ (of the norm 1 ) is mapped by (1.22) to the element $(d x / b)$ of the norm $\|d x / b\|^{2}=b^{-1}$. The element $h=1^{-1} \otimes(d x / b)$, corresponding to the element $1 \in \mathbb{C}=\operatorname{det} H^{\bullet}((0, b])$, has the norm $b^{-1}$. So the equality (1.21) claims that

$$
\log 2=-\log b-\log (\pi / b)-2 \zeta^{\prime}(0) .
$$

Thus the equality $\zeta^{\prime}(0)=-2^{-1} \log (2 \pi)$ is a particular case ${ }^{4}$ of Theorem 1.2.

The natural $L_{2}$-norm on $\oplus_{j} D R^{\bullet}\left(\bar{M}_{j}\right)$ is defined by

$$
\left(v_{1}, v_{1}\right):=\int_{M}\left(v_{1} \wedge * \bar{v}_{1}\right)
$$

where $\left(v_{1} \wedge * \bar{v}_{1}\right)$ is a real density on $M$, corresponding to $v_{1} \wedge * \bar{v}_{1}$.

Lemma 1.1. The Green functions $G_{\nu}$ for the Laplacians $\Delta_{\nu}^{\bullet}$ provide us with the homotopy operator in the complex $D R^{\bullet}\left(M_{\nu}, Z\right)$

$$
K_{\nu}:=\delta G_{\nu}
$$

between the orthogonal projection operator $p_{\mathcal{H}}: D R^{\bullet}\left(M_{\nu}, Z\right) \rightarrow \operatorname{Ker}\left(\Delta_{\nu}^{\bullet}\right)$ and the identity operator on $D R^{\bullet}\left(M_{\nu}, Z\right)$. The following equality holds in $D R^{\bullet}\left(M_{\nu}, Z\right)$ :

$$
d K_{\nu}+K_{\nu} d=\mathrm{id}-p_{\mathcal{H}}
$$

Proof. The Green function for $\Delta_{\nu}^{\bullet}$ maps the $L_{2}$-completion $\left(D R^{\bullet}(M)\right)_{2}$ of $D R^{\bullet}(M)^{5}$ into the $\operatorname{Dom}\left(\Delta_{\nu}^{\bullet}\right)$ (Theorem 3.1). The $\operatorname{Dom}\left(\Delta_{\nu}^{\bullet}\right)$ is defined as the domain of definition $D\left(\Delta_{\nu}^{\bullet}\right)$ for $\Delta_{\nu}^{\bullet}$ in $D R^{\bullet}\left(M_{\nu}, Z\right)$ completed with respect to the graph topology norm $\|\omega\|_{\text {graph }}^{2}:=\|\omega\|_{2}^{2}+\left\|\Delta_{\nu}^{\bullet} \omega\right\|_{2}^{2}$ for $\omega \in D\left(\Delta_{\nu}^{\bullet}\right)\left(\right.$ where $\|\omega\|_{2}^{2}:=(\omega, \omega)$ is the $L_{2^{-}}$ norm (1.23)). The Green function $G_{\nu}$ maps $D R^{\bullet}\left(M_{\nu}, Z\right)$ into $D\left(\Delta_{\nu}^{\bullet}\right)$ (since, by

\footnotetext{
${ }^{4}$ In this paper the proofs of the equality (1.18), of Theorem 1.1, and of the equality (1.12) with $c_{0}=1$ do not use the Lerch formula. So we have obtained (by the way) a new proof of the Lerch formula.

${ }^{5}\left(D R^{\bullet}(M)\right)_{2}$ coincides with the $L_{2}$-completion of $D R^{\bullet}\left(M_{\nu}, Z\right)$ and with the $L_{2}$-completion of $\oplus_{j} D R^{\bullet}\left(\bar{M}_{j}\right)$.
} 
Theorem 3.1, $\Delta_{\nu}^{\bullet}$ is a nonnegative elliptic differential operator with elliptic boundary conditions). The definition of the Green function claims that

$$
\Delta_{\nu}^{\bullet} G_{\nu}=\mathrm{id}-p_{\mathcal{H}}
$$

on $\left(D R^{\bullet}(M)\right)_{2}$ (where $\Delta_{\nu}^{\bullet} \omega$ for $\omega \in \operatorname{Dom}\left(\Delta_{\nu}^{\bullet}\right)$ is defined as $\lim _{i} \Delta_{\nu}^{\bullet} \omega_{i}$ for $\omega_{i} \in D\left(\Delta_{\nu}^{\bullet}\right)$, $\left.\left\|\omega-\omega_{i}\right\|_{\text {graph }}^{2} \rightarrow 0\right)$. In particular, this equality holds on $D R^{\bullet}\left(M_{\nu}, Z\right) \subset\left(D R^{\bullet}(M)\right)_{2}$.

The $D\left(\Delta_{\nu}^{\bullet}\right) \subset\left(D R^{\bullet}(M)\right)_{2}$ is defined as follows. The adjoint to $d_{\nu}$ operator $\delta_{\nu}$ in $\oplus_{j} D R^{\bullet}\left(\bar{M}_{j}\right)$ is defined on elements $v_{2}=\left(\omega_{1}, \omega_{2}\right)$, where $\omega_{k}$ are smooth differential forms on $\bar{M}_{k}$ and the linear functional

$$
l_{v_{2}}\left(v_{1}\right)=<d v_{1}, v_{2}>=\int_{M}\left(d v_{1} \wedge * \bar{v}_{2}\right)
$$

is continuous in $D R^{\bullet}\left(M_{\nu}, Z\right)$ with respect to the $L_{2}$-norm $(1.23)$ of $v_{1} \in D R^{\bullet}\left(M_{\nu}, Z\right)$. For such an element $v_{2}=\left(\omega_{1}, \omega_{2}\right)$ the form $* v_{2}=\left(* \omega_{1}, * \omega_{2}\right)$ has the zero geometrical restriction to $\partial M \backslash Z$, and the following transmission condition has to hold on $N$ for $v_{2}$ :

$$
\beta i_{N, 1}^{*}\left(* \omega_{1}\right)=\alpha i_{N, 2}^{*}\left(* \omega_{2}\right),
$$

where $i_{N, k}: \quad N \subset \partial M_{k} \hookrightarrow M_{k}$. (These boundary conditions for $v_{2}$ are consequences of Stokes' formula.)

The domain $D\left(\Delta_{\nu}^{\bullet}\right) \subset D R^{\bullet}\left(M_{\nu}, Z\right)$ is defined as the set of $\omega \in D R^{\bullet}\left(M_{\nu}, Z\right)$ such that

$$
\omega \in D\left(\delta_{\nu}\right), \quad d \omega \in D\left(\delta_{\nu}\right), \quad \delta \omega \in D R^{\bullet}\left(M_{\nu}, Z\right) .
$$

Note that $d G_{\nu} \omega=G_{\nu} d \omega$ for $\omega \in D R^{\bullet}\left(M_{\nu}, Z\right)$ (this equality follows from Stokes' formula). Hence the identity (1.25) can be represented on $D R^{\bullet}\left(M_{\nu}, Z\right)$ as

$$
K d+d K=i d-p_{\mathcal{H}}
$$

Thus the lemma is proved.

Corollary 1.1. The homotopy operator (1.24) defines a canonical identification between the cohomology $H^{\bullet}\left(D R\left(M_{\nu}, Z\right)\right)$ and the space of harmonic forms $\operatorname{Ker}\left(\Delta_{\nu}\right)$.

Let for simplicity $g_{M}$ be a direct product metric near $N$. Let the Dirichlet boundary conditions be given on a union $Z$ of some connected components of $\partial M$ and the Neumann conditions be given on $\partial M \backslash Z$. Then the following holds.

Proposition 1.1. The eigenforms of $\Delta\left(M, Z ; g_{M}\right)$ are the same as the eigenforms of $\Delta_{1,1}$. 
Proof. Let $\nu$ be equal to $(1,1)$. The conditions (1.27) for $\omega=\left(\omega_{1}, \omega_{2}\right) \in D R^{\bullet}\left(M_{1,1}, Z\right)$ are equivalent on $N$ to the following ones:

$$
\begin{aligned}
i_{N, 1}^{*} \omega_{1} & =i_{N, 2}^{*} \omega_{2}, & i_{N, 1}^{*}\left(* \omega_{1}\right) & =i_{N, 2}^{*}\left(* \omega_{2}\right), \\
i_{N, 1}^{*}\left(* d \omega_{1}\right) & =i_{N, 2}^{*}\left(* d \omega_{2}\right), & i_{N, 1}^{*}\left(* d * \omega_{1}\right) & =i_{N, 2}^{*}\left(* d * \omega_{2}\right),
\end{aligned}
$$

where $*$ is the star-operator for the Riemannian metric $g_{M}$.

The equalities (1.28) claim that the restrictions to $N$ of the forms $\omega_{1}$ and $\omega_{2}$ are the same (i.e., they are the same smooth sections of $\left.\wedge^{\bullet} T^{*} M\right|_{N}$ ). The equalities (1.28) and (1.29) are equivalent to the assertion that the following pairs of forms have the same restrictions to $N$ (as the smooth sections of $\left.\wedge^{\bullet} T^{*} M\right|_{N}$ ):

$$
\left\{d \omega_{1}, d \omega_{2}\right\}, \quad\left\{\delta \omega_{1}, \delta \omega_{2}\right\}, \quad\left\{\omega_{1}, \omega_{2}\right\} .
$$

Any eigenform for $\Delta^{\bullet}\left(M, Z ; g_{M}\right)$ belongs to $D\left(\Delta_{1,1}^{\bullet}\right)$. So it is an eigenform for $\Delta_{1,1}^{\bullet}$. Let $\omega=\left(\omega_{1}, \omega_{2}\right) \in D\left(\Delta_{1,1}^{\bullet}\right)$ be an eigenform for $\Delta_{1,1}$ :

$$
\Delta_{1,1}^{\bullet} \omega=\left(\Delta^{\bullet} \omega_{1}, \Delta^{\bullet} \omega_{2}\right)=\lambda\left(\omega_{1}, \omega_{2}\right) .
$$

Then ${ }^{6} \omega_{k}$ are $C^{\infty}$-forms on $\bar{M}_{k}$ and (as it follows from $(1.30),(1.31)$ ) the restrictions of the following pairs of forms are the same as the sections of $\left.\wedge^{\bullet} T^{*} M\right|_{N}$ (for $k=$ $0,1,2 \ldots)$ :

$$
\left\{\Delta^{k} \omega_{1}, \Delta^{k} \omega_{2}\right\}, \quad\left\{d \Delta^{k} \omega_{1}, d \Delta^{k} \omega_{2}\right\}, \quad\left\{\delta \Delta^{k} \omega_{1}, \delta \Delta^{k} \omega_{2}\right\}
$$

So $\omega=\left(\omega_{1}, \omega_{2}\right)$ is a $C^{\infty}$-form on $M=M_{1} \cup_{N} M_{2}$. In fact, it follows from (1.28) and from the identity of the restrictions to $N$ of $\Delta \omega_{1}$ and $\Delta \omega_{2}$ that $\left(\Delta_{I} \otimes\right.$ id) $\omega_{k}$ have (for $k=1,2$ ) the same restrictions from $M_{k}$ to $N$. (The Laplacian $\Delta$ is equal to $\operatorname{id}_{I} \otimes \Delta_{N}+\Delta_{I} \otimes \mathrm{id}_{N}$ with respect to the direct product structure $I \times N$ in the neighborhood of $N=0 \times N \hookrightarrow I \times N \hookrightarrow M, 0 \in I \backslash \partial I$.) Hence, according to (1.28) and (1.29), the 2-jets of $\omega_{1}$ and of $\omega_{2}$ are the same on $N$. The identity between the $(2 k+1)$-jets of $\omega_{k}$ on $N$ follows (by induction) from (1.32). Thus, $\omega$ is an eigenform for $\Delta_{M}: \Delta_{M} \omega=\lambda \omega$. The proposition is proved.

1.3. Properties of analytic and combinatorial torsion norms. One of the main properties of the analytic torsion norm is as follows. Let $M$ be a manifold $M_{1} \times M_{2}$ with a direct product metric. One of these Riemannian manifolds, for instance $M_{1}$, can have a nonempty boundary $\partial M_{1}$. In this case let $g_{M_{1}}$ be a direct product metric near $\partial M_{1}$, and let the Dirichlet boundary conditions be given on the components $Z=Z_{1} \times M_{2}$ of $\left(\partial M_{1}\right) \times M_{2}=\partial\left(M_{1} \times M_{2}\right)$. Let the Neumann boundary conditions be given on $\partial\left(M_{1} \times M_{2}\right) \backslash\left(Z_{1} \times M_{2}\right)=\left(\partial M_{1} \backslash Z_{1}\right) \times M_{2}$.

\footnotetext{
${ }^{6}$ All the eigenforms of $\Delta_{\nu}^{\bullet}$ (for $\left.\nu \in \mathbb{R}^{2} \backslash(0,0)\right)$ are $C^{\infty}$-smooth on $\bar{M}_{k}$, as it follows from Theorem 3.1.
} 
The Künneth formula for the cohomology claims that

$$
H^{j}(M, Z)=\oplus_{i+k=j} H^{i}\left(M_{1}, Z_{1}\right) \otimes H^{k}\left(M_{2}\right) .
$$

So the determinant of the cohomology of $D R^{\bullet}(M, Z)$ is the tensor product

$$
\operatorname{det} H^{\bullet}(M, Z)=\left(\operatorname{det} H^{\bullet}\left(M_{1}, Z_{1}\right)\right)^{\chi\left(M_{2}\right)} \otimes \operatorname{det} H^{\bullet}\left(M_{2}\right)^{\chi\left(M_{1}, Z_{1}\right)} .
$$

Proposition 1.2. The identification (1.34) induces the isomorphism of the analytic torsion norm $T_{0}(M, Z)$ with the tensor product

$$
T_{0}(M, Z)=T_{0}\left(M_{1}, Z_{1}\right)^{\otimes \chi\left(M_{2}\right)} \otimes T_{0}\left(M_{2}\right)^{\otimes \chi\left(M_{1}, Z_{1}\right)},
$$

where $\chi\left(M_{1}, Z_{1}\right), \chi\left(M_{2}\right)$ are the Euler characteristics.

Remark 1.1. It is shown above that the analytic torsion norms $T_{0}(M, Z), T_{0}\left(M_{1}, Z_{1}\right)$, and $T_{0}\left(M_{2}\right)$ are independent of Riemannian metrics $g_{M}, g_{M_{k}}$ which are supposed to be direct product metrics near $\partial M, \partial M_{1}$. So, if the equality (1.35) holds for a direct product metric on $\left(M_{1}, \partial M_{1}\right) \times M_{2}$, (where $g_{M_{1}}$ is a direct product metric near $\partial M_{1}$ ) then this equality holds for any metric $g_{M}$ (which is supposed to be a direct product metric near $\partial M$ ).

Proof. The scalar analytic torsion $T(M)$ for a direct product metric on $M=M_{1} \times M_{2}$ is equal to

$$
T(M, Z)=T\left(M_{1}, Z_{1}\right)^{\chi\left(M_{2}\right)} T\left(M_{2}\right)^{\chi\left(M_{1}, Z_{1}\right)} .
$$

This statement is proved in [RS], Theorem 2.5 , in the case of an acyclic local system over $M_{1}$. In the general case, (1.36) follows from the proof of Theorem 2.5 in [RS] and from the following equality (where $\lambda \neq 0, m\left(i, \lambda, M_{2}\right)$ is the dimension of the $\lambda$-eigenspace for $\left.\Delta_{M_{2}, i}, m\left(j, 0, M_{1}\right):=\operatorname{dim} \operatorname{Ker} \Delta_{M_{1}, Z_{1} ; j}\right)$ :

$$
\sum(-1)^{i+j}(i+j) m\left(i, \lambda, M_{2}\right) m\left(j, 0, M_{1}\right)=\left(\sum(-1)^{i} i m\left(i, \lambda, M_{2}\right)\right) \chi\left(M_{1}, Z_{1}\right),
$$

which holds, since the alternating sum over $i$ of $m\left(i, \lambda, M_{2}\right)$ is equal to zero (for any nonzero $\lambda$ ).

For such a metric on $M$ the following canonical identifications are the isometries between the natural Hilbert structure on the space of harmonic forms $\operatorname{Ker} \Delta_{j}(M, Z)$ and the tensor products (and the direct sums) of the Hilbert structures on harmonic forms for $\Delta_{\boldsymbol{\bullet}}\left(M_{1}, Z_{1}\right)$ and $\Delta_{\boldsymbol{\bullet}}\left(M_{2}\right)$ :

$$
\operatorname{Ker} \Delta_{j}(M, Z)=\oplus_{i+k=j} \operatorname{Ker} \Delta_{i}\left(M_{1}, Z_{1}\right) \otimes \operatorname{Ker} \Delta_{k}\left(M_{2}\right) .
$$

These Hilbert structures induce the norms on $\operatorname{det} H^{\bullet}(M, Z)=\operatorname{det} \operatorname{Ker} \Delta_{\bullet}\left(M, Z ; g_{M}\right), \operatorname{det} H^{\bullet}\left(M_{1}, Z_{1}\right)=\operatorname{det} \operatorname{Ker} \Delta_{\bullet}\left(M_{1}, Z_{1} ; g_{M_{1}}\right)$ 
and on $\operatorname{det} H^{\bullet}\left(M_{2}\right)=\operatorname{det} \operatorname{Ker} \Delta_{\bullet}\left(M_{2}, g_{M_{2}}\right)$ such that the identification (1.34) of the determinant lines is an isometry:

$$
\|\cdot\|_{\operatorname{det} \operatorname{Ker} \Delta_{M}}^{2}=\left(\|\cdot\|_{\operatorname{det} \operatorname{Ker} \Delta_{M_{1}, Z_{1}}}^{2}\right)^{\chi\left(M_{2}\right)}\left(\|\cdot\|_{\operatorname{det} \operatorname{Ker} \Delta_{M_{2}}}^{2}\right)^{\chi\left(M_{1}, Z_{1}\right)} .
$$

The equality (1.35) follows from (1.36),(1.34), and (1.38).

The following lemma makes it possible to use the variations on $\nu$ in the proof of Theorem 1.1. Let $\left(M, g_{M}\right)$ be a compact Riemannian manifold with a smooth boundary $\partial M$ and let $N \hookrightarrow M \backslash \partial M$ be a smooth closed codimension one submanifold of $M$ with a trivial normal bundle $\left(\left.T M\right|_{N}\right) / T N$ such that $M=M_{1} \cup_{N} M_{2}$ is obtained by gluing two its pieces $M_{1}$ and $M_{2}$ along $N$. Let $g_{M}$ be a direct product metric near $\partial M$ and near $N$. Let $Z$ be a union of some connected components of $\partial M$ where the Dirichlet boundary conditions are given and let the Neumann boundary conditions be given on $\partial M \backslash N$.

Lemma 1.2. Let us suppose that the norm $\varphi_{\nu}^{a n} T_{0}\left(M_{\nu}, Z\right)$ is independent of $\nu \in$ $\mathbb{R}^{2} \backslash(0,0)$ for any such $\left(M, g_{M}, N, Z\right)^{7}$ (where the identification $\varphi_{\nu}^{a n}$ is defined by the exact sequence (1.14) of the de Rham complexes and by Lemma 1.1). Then the factor $c_{0}$ in the gluing formula (1.12) for $\varphi_{\nu}^{a n} T_{0}\left(M_{\nu}, Z\right)$ is equal to one.

Remark 1.2. Theorem 1.1 is a direct consequence of Lemma 1.2 and of the assertion that $\varphi_{\nu}^{a n} T_{0}\left(M_{\nu}, Z\right)$ is independent of $\nu \in \mathbb{R}^{2} \backslash(0,0)$. Indeed, $T_{0}\left(M_{1,1}, Z\right)$ coincides with $T_{0}(M, Z)$ (according to Proposition 1.1) and the identifications $\varphi_{\nu}^{a n}$ and $\varphi_{a n}$ are the same. Hence the formula (1.12), where $c_{0}$ is equal to one and $\nu=(1,1)$, is the gluing formula of Theorem 1.1.

Remark 1.3. The assertion that the norm $\varphi_{\nu}^{a n} T_{0}\left(M_{\nu}, Z\right)$ does not depend on $\nu$ is equivalent to the independence of $\nu$ of the factor $c_{0}$ in (1.12).

Proof. The factor $c_{0}$ in (1.12) lies in $\mathbb{R}_{+}$. If $c_{0}$ is independent of $\nu$ for $\left(M, g_{M}, N, Z\right)$ then

$$
\varphi_{1,0}^{a n} T_{0}\left(M_{1,0}, Z\right)=\varphi_{0,1}^{a n} T_{0}\left(M_{0,1}, Z\right) .
$$

It follows from (1.39) and from (1.16), (1.19), and (1.14) that there are the equalities with the same positive constant $c_{0}$ as in (1.12) for $\left(M, g_{M}, N, Z\right)$ (where $Z_{k}:=Z \cap$ $\left.\partial M_{k}\right)$ :

$$
\begin{aligned}
& \varphi_{a n} T_{0}\left(M_{1}, Z_{1}\right)=c_{0} T_{0}\left(M_{1}, Z_{1} \cup N\right) \otimes T_{0}(N), \\
& \varphi_{a n} T_{0}\left(M_{2}, Z_{2}\right)=c_{0} T_{0}\left(M_{2}, Z_{2} \cup N\right) \otimes T_{0}(N) .
\end{aligned}
$$

\footnotetext{
${ }^{7}$ The equivalent formulation is as follows. Let $M$ be obtained by gluing along $N$, i.e., $M=$ $M_{1} \cup_{N} M_{2}$, and let it be equiped with a Riemannian metric $g_{M}$, which is a direct product metric near $\partial M$ and near $N$. Then it is supposed that the norm $\varphi_{\nu}^{a n} T_{0}\left(M_{\nu}, Z\right)$ is independent of $\nu \in \mathbb{R}^{2} \backslash(0,0)$.
} 
We can conclude from (1.40) and (1.41) that the factor $c_{0}=c_{0}\left(N, g_{N}\right)$ is defined by $N, g_{N}$ and that it does not depend on $M_{1}, M_{2}, M$, and $g_{M}$ (it is independent also of $\nu)$.

Let $M_{1}$ in (1.40) be a manifold $M_{1}=N \times I$ with a direct product metric. Then $\partial M_{1}=N \cup N$ and (1.40) claims in this case that

$$
\varphi_{a n} T_{0}(N \times I)=c_{0}\left(N, g_{N}\right)^{2} T_{0}(N \times I, N \times \partial I) \otimes T_{0}(N)^{\otimes 2},
$$

where the identification $\varphi_{a n}$ is defined by the exact sequence (1.20). It follows from (1.42) and from the multiplicative property (1.35) that

$$
T_{0}(N)^{\chi(I)} \otimes T_{0}(I)^{\chi(N)}=c_{0}^{2} T_{0}(N)^{\chi(I, \partial I)} \otimes T_{0}(I, \partial I)^{\chi(N)} \otimes T_{0}(N)^{2},
$$

where $c_{0}:=c_{0}\left(N, g_{N}\right)$ depends on $N$ and on $g_{N}$ only. Then the following equality is a consequence of (1.43) and of the identification (1.19) (defined by the exact sequence $(1.20))$ :

$$
T_{0}(I)^{\chi(N)}=c_{0}\left(N, g_{N}\right)^{2} T_{0}(I, \partial I)^{\chi(N)} \otimes T_{0}(\partial I)^{\chi(N)} .
$$

Note that $T_{0}(\partial I)$ is the standart norm on $\operatorname{det} H^{\bullet}(\partial I)$ which is canonically identified with $\mathbb{C}$ (up to a possible factor $(-1)$ in the identification). Namely $\|1\|_{T_{0}}^{2}=1$ for $1 \in \mathbb{C}$. An immediate consequence of the equality (1.41) for $M_{1}=I, N=\partial I$ and of (1.44) is the following:

$$
c_{0}\left(N, g_{N}\right)^{2}=c_{0}(\partial I)^{\chi(N)} .
$$

Hence, it is enough to prove that $c_{0}(\partial I)=1$, and it will be done now.

Let $I$ be an interval $[0, a]$. The scalar analytic torsions for $I$ and for $(I, \partial I)$ are equal: $T(I)=T(I, \partial I)$, since

$$
\begin{aligned}
\zeta_{1}(s ; I) & =\zeta_{0}(s ; I, \partial I), \\
\zeta_{1}(s ; I, \partial I) & =\zeta_{0}(s ; I, \partial I),
\end{aligned}
$$

(where $\zeta_{j}(s ; M, Z)$ is the $\zeta$-function of the Laplacian on $\left(D R^{j}(M, Z), g_{M}\right)$ ). The equality (1.47) follows from the identification of the eigenforms, defined by the exterior derivative $d$, and the equality (1.46) follows from the identification of the eigenforms defined by the Riemannian $*$ on $I$.

The cohomology exact sequence for the pair $(I, \partial I)$ is

$$
0 \rightarrow H^{0}(I) \rightarrow H^{0}(\partial I) \rightarrow H^{1}(I, \partial I) \rightarrow 0 .
$$

The complex (1.48) is acyclic and so the determinant $D$ of its cohomology is canonically isomorphic to $\mathbb{C}$. The components of (1.48) are equiped with the natural Hilbert structures (because they are the spaces of harmonic forms on the interval $I \subset \mathbb{R}$ with the standart metric). Hence, there is the induced norm $\|\cdot\|_{D}$ on $D=\mathbb{C}$. We have to prove that $\|1\|_{D}^{2}=1$ for $1 \in \mathbb{C}=D$. This equality is equavalent to the assertion that $c_{0}(\partial I)$ is equal to one. 
The norm of the element $a^{-1 / 2} \cdot 1 \in D R^{0}(I)$ is equal to 1 . (It is a harmonic form and it represents an element from $\left.H^{0}(I)\right)$. Its image in $H^{0}(\partial I)$ is as follows:

$$
-a^{-1 / 2} \cdot[0]+a^{-1 / 2} \cdot[a] \in H^{0}(\partial I)=H^{0}(0 \cup a) .
$$

The norm of the element $a^{-1 / 2} \cdot d x \in D R^{1}(I, \partial I)$ is equal to 1 in $H^{1}(I, \partial I)$ and an element $-a^{1 / 2} \cdot[0]$ is mapped by the differential of the exact sequence (1.48) to the harmonic form $a^{-1 / 2} d x \in H^{1}(I, \partial I)$. (The arrows in (1.48) are of the topological nature. So the latter statement is obtained using

$$
a^{1 / 2}=\int_{[0, a]} a^{-1 / 2} d x=\left(a^{-1 / 2} d x,(I, \partial I)\right),
$$

where $(I, \partial I)$ is the fundamental class of $H_{1}(I, \partial I)$.)

The corresponding volume element $\left(-a^{-1 / 2}[0]+a^{-1 / 2}[a]\right) \wedge\left(-a^{1 / 2}[0]\right)=[0] \wedge[a]$ in $\operatorname{det} H^{0}(\partial I)$ is an element with the norm one. Hence, $c_{0}(\partial I)=1$. The equality $c_{0}\left(N, g_{N}\right)=1$ (for a union $N$ of some connected components of $\partial M_{1}$ ) follows from the equality $c_{0}(\partial I)=1$ and from (1.45). The lemma is proved.

Let $M=M_{1} \cup_{N} M_{2}$ be obtained by gluing $M_{1}$ and $M_{2}$ along $N$ (as in Theorem 1.1), and let $X$ be a smooth triangulation of $M$ such that $M_{k}$ and $N$ are invariant under $X$. Namely $X=X_{1} \cup_{W} X_{2}$, where $X_{k}$ is a smooth triangulation of a manifold $M_{k}$ with a smooth boundary $\partial M_{k}=N \cup\left(\partial M \cap \bar{M}_{k}\right)$. (Here $N \subset M$ is a smooth closed manifold of codimension one in $M$ such that $N$ divides $M$ in two pieces $M_{1}$ and $M_{2}$ as in Theorem 1.1, $N \cap \partial M=\emptyset$, and $W:=X \cap N=X_{k} \cap N$.)

Let $Z$ be a union of the connected components of $\partial M$, where the Dirichlet boundary conditions are given. Set $V:=X \cap Z, Z_{k}:=\partial M_{k} \cap Z, V_{k}:=X_{k} \cap Z_{k}$. The exact sequence of cochain complexes

$$
0 \rightarrow \oplus_{k=1,2} C^{\bullet}\left(X_{k}, W \cup V_{k}\right) \rightarrow C^{\bullet}(X, V) \stackrel{i_{N}^{*}}{\longrightarrow} C^{\bullet}(W) \rightarrow 0
$$

(where the left arrow is the natural inclusion and the right arrow is the geometrical restriction of cochains) provides us with the identification

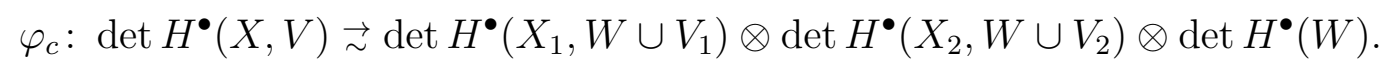

By the definition of the combinatorial torsion norm on the determinant line (determined by the prefered basises of the basic cochains) the following statement holds.

Proposition 1.3. Under the conditions above, the combinatorial torsion norms are equal:

$$
\varphi_{c} \tau_{0}(X, V)=\tau_{0}\left(X_{1}, W \cup V_{1}\right) \otimes \tau_{0}\left(X_{2}, W \cup V_{2}\right) \otimes \tau_{0}(W)
$$

This combinatorial equality is analogous to the gluing formula of Theorem 1.1. But it is necessary to note as follows. 
Remark 1.4. The formulas (1.50) and (1.8) correspond to the different identifications $\varphi_{c}$ and $\varphi_{a n}=\varphi_{1,1}^{a n}$ between one pair of the canonically identified ${ }^{8}$ one-dimensional $^{2}$ spaces

$$
\operatorname{det} H^{\bullet}(X, V)=\operatorname{det} H^{\bullet}(M, Z),
$$

and the triple tensor products of three other pairs of the canonically identified spaces $\operatorname{det} H^{\bullet}\left(X_{k}, W \cup V_{k}\right)=\operatorname{det} H^{\bullet}\left(M_{k}, N \cup Z_{k}\right), \quad \operatorname{det} H^{\bullet}(W)=\operatorname{det} H^{\bullet}(N)$.

(Note that $\varphi_{c}$ is defined by the exact sequence (1.49), where the right arrow $i_{N}^{*}$ is the restriction of the cochains. However, in the exact sequence (1.10), which defines $\varphi_{a n}$, the right arrow is equal to $\sqrt{2} i_{N}^{*}$ for the common geometrical restriction $i_{N}^{*}$ to $N$ of pairs $\omega=\left(\omega_{1}, \omega_{2}\right)$ of smooth differential forms $\omega_{k}$ on $\bar{M}_{k}$ such that $i_{N, 1}^{*} \omega_{1}=i_{N, 2}^{*} \omega_{2}$.)

Let $X$ be a smooth triangulation of a compact manifold with boundary $(M, \partial M)$. Let $Z$ and $Y$ be disjoint unions of some connected components of $\partial M$ such that $Z \cap Y=\emptyset$. Let $V=X \cap Z, F=X \cap Y$. Then the exact sequence

$$
0 \rightarrow C^{\bullet}(X, V \cup F) \rightarrow C^{\bullet}(X, V) \rightarrow C^{\bullet}(F) \rightarrow 0
$$

(where the left arrow is the natural inclusion of cochains and the right arrow is the restriction of cochains) defines the identification

$$
\varphi_{c}^{F}: \operatorname{det} H^{\bullet}(X, V) \rightleftharpoons \operatorname{det} H^{\bullet}(X, V \cup F) \otimes \operatorname{det} H^{\bullet}(F)
$$

The following assertion is an immediate consequence of the definition of the combinatorial torsion norm.

Proposition 1.4. The combinatorial torsion norm of $(X, V)$ is equal to the tensor product of the following combinatorial torsion norms:

$$
\varphi_{c} \tau_{0}(X, V)=\tau_{0}(X, V \cup F) \otimes \tau_{0}(F) .
$$

This combinatorial equality is similar to the gluing formula of Theorem 1.2.

Let $e(M, Z)$ be the logarithm of the ratio between the analytic and the combinatorial torsion norms:

$$
e(M, Z):=\log _{2}\left(T_{0}(M, Z) / \tau_{0}(X, V)\right)
$$

(where $T_{0}(M, Z) / \tau_{0}(X, V):=\|l\|_{T_{0}(M, Z)}^{2} /\|l\|_{\tau_{0}(X, V)}^{2}$ for an arbitrary nonzero element $l$ of the determinant line $\left.\operatorname{det} H^{\bullet}(M, Z)=\operatorname{det} H^{\bullet}(X, V)\right)$.

Remark 1.5. It is proved above that $e(M, \partial M)$ does not depend on a metric $g_{M}$, if $g_{M}$ is a direct product metric near $\partial M$.

\footnotetext{
${ }^{8}$ The cohomology are identified according to the de Rham theorem by the integration over the simplexes of $X$ of the corresponding differential forms. The spaces of harmonic forms $\operatorname{Ker} \Delta_{\bullet}(M, Z)$ and $\operatorname{Ker} \Delta_{\bullet}\left(M_{1,1}, Z\right)$ are canonically identified by Proposition 1.1.
} 
Lemma 1.3. 1. Let $\left(S, g_{S}\right)$ be a closed Riemannian manifold. Then the following identity holds, if $g_{M \times S}$ is a direct product metric near $\partial(M \times S)=\partial M \times S$ :

$$
e(M \times S, Z \times S)=\chi(M, Z) e(S)+e(M, Z) \chi(S)
$$

$(\chi(M, Z)$ is the relative Euler characteristic of $M$ modulo $Z \subset \partial M)$.

2. Let $Y$ be a union of some connected components of $\partial M \backslash Z$. Then

$$
e(M, Z)=e(M, Y \cup Z)+e(Y) .
$$

Proof. The equality (1.52) follows from Theorem 1.2 and from Proposition 1.4. (In this case, $\varphi_{c}=\varphi_{a n}$.) The equality (1.51) follows from Proposition 1.2 and from the multiplicative property of the combinatorial torsion norms. Namely let $K$ be a smooth triangulation of $S$ and let $V=X \cap Z$. Then the identification of the determinants of the cohomology defined by (1.33) and (1.34) is an isometry of the combinatorial torsion norms:

$$
\tau_{0}(X \times K, V \times K)=\tau_{0}(X, V)^{\chi(K)} \otimes \tau_{0}(K)^{\chi(X, V)} .
$$

The same identification of the cohomology is the isometry (1.35) of the analytic torsion norms, if the metric $g_{M \times S}$ is a direct product metric near $\partial(M \times S)$. Hence, the identity (1.51) holds for such metrics $g_{M \times S}$.

Remark 1.6. It follows from (1.52) and from Remark 1.5 that $e(M, Z)$ does not depend on $g_{M}$ for any union $Z$ of the connected components of $\partial M$ (in particular for $Z=\emptyset)$.

\subsection{Generalized Ray-Singer conjecture.}

1.4.1. Properties of the ratio of the analytic and the combinatorial torsion norms. Lemma 1.2 claims that Theorem 1.1 follows from (1.12) with $c_{0}$ independent of $\nu$. So it is enough to prove that the norm $\varphi_{\nu}^{a n} T_{0}\left(M_{\nu}, Z\right)$ is independent of $\nu \in \mathbb{R}^{2} \backslash(0,0)$ (under the same conditions on $M, g_{M}, N$, and $Z$ as in (1.12) and in Lemma 1.2). The latter assertion is proved in Section 2. In the remaining part of Section 1 we prove a generalization of the Ray-Singer conjecture for manifolds with boundary (and with the transmission condition (1.13) on the interior boundary) using the gluing formula of Theorem 1.1. This formula has the following consequence.

Let $M=M_{1} \cup_{N} M_{2}$ be obtained by gluing $M_{1}$ and $M_{2}$ along $N$.

Lemma 1.4. Under the conditions of Lemma 1.2, on $\left(g_{M}, N, Z\right)$ the following holds:

$$
e(M, Z)=e\left(M_{1}, Z_{1} \cup N\right)+e\left(M_{2}, Z_{2} \cup N\right)+e(N)-\chi(N) .
$$


Proof. This identity is an immediate consequence of Theorem 1.1 and of the following commutative diagram:

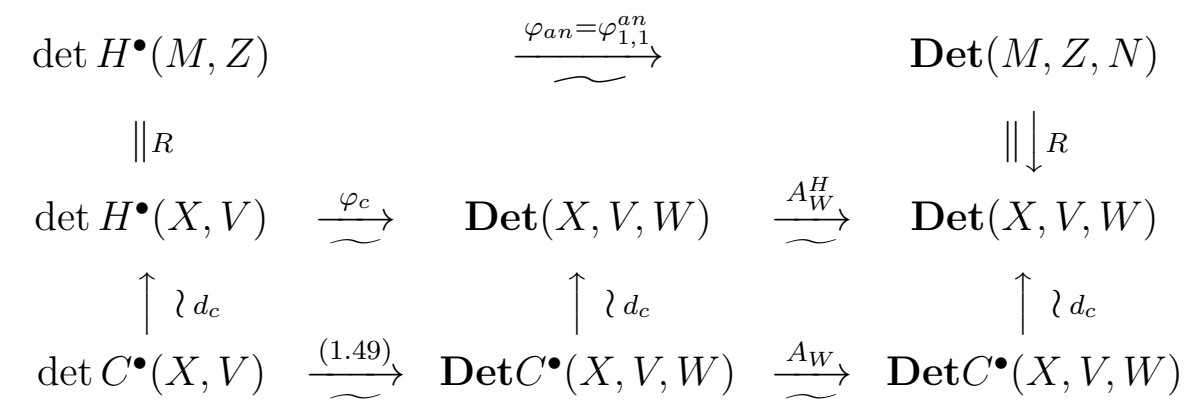

Here

$$
\begin{gathered}
\operatorname{Det}(M, Z, N):=\left(\otimes_{k=1,2} \operatorname{det} H^{\bullet}\left(M_{k}, N \cup Z_{k}\right)\right) \otimes \operatorname{det} H^{\bullet}(N), \\
\operatorname{Det}(X, V, W):=\left(\otimes_{k=1,2} \operatorname{det} H^{\bullet}\left(X_{k}, W \cup V_{k}\right)\right) \otimes \operatorname{det} H^{\bullet}(W), \\
\operatorname{Det} C^{\bullet}(X, V, W):=\left(\otimes_{k=1,2} \operatorname{det} C^{\bullet}\left(X_{k}, W \cup V_{k}\right)\right) \otimes \operatorname{det} C^{\bullet}(W), \\
A_{W}:=\operatorname{id}_{X} \oplus \sqrt{2} \operatorname{id}_{W} \in \operatorname{Aut}\left(\oplus_{k=1,2} C^{\bullet}\left(X_{k}, W \cup V_{k}\right)\right) \oplus \operatorname{Aut} C^{\bullet}(W),
\end{gathered}
$$

$A_{W}^{H}$ is the induced by $A_{W}$ operator on the determinant of the cohomology,

$R$ is the identification induced by the integration of differential forms over the simplexes of $X$ (by the de Rham theorem),

$\varphi_{c}$ and $\varphi_{a n}$ are the identifications induced by (1.49) and by (1.10) in a view of Proposition 1.1 .

The commutativity of (1.53) follows from the commutativity of the diagram

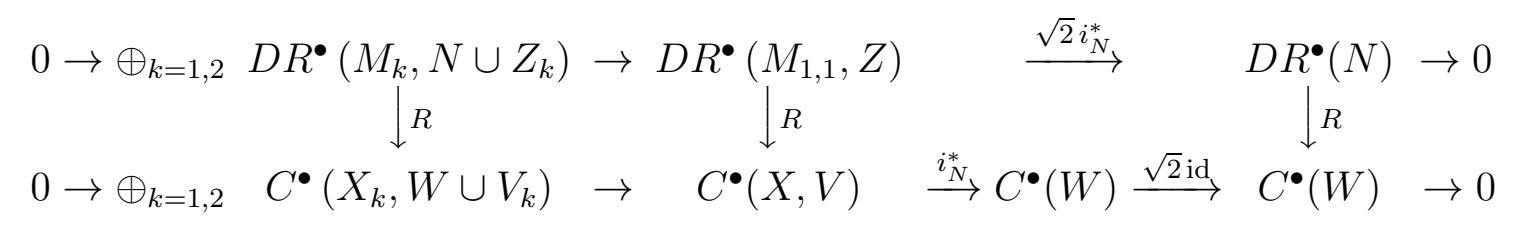

The induced action of $\sqrt{2}$ id on $l_{W} \in \operatorname{det} C^{\bullet}(W)$ is $l_{W} \rightarrow 2^{-\chi(W) / 2} l_{W}$ (where $\chi(W)=\chi(N)$ is the Euler characteristic). So the induced action of $A_{W}$ and of $A_{W}^{H}$ on $l \in \operatorname{Det}(M, Z, N)=\left(\otimes_{k=1,2} \operatorname{det} C^{\bullet}\left(X_{k}, W \cup V_{k}\right)\right) \otimes \operatorname{det} C^{\bullet}(W)$ is

$$
l \rightarrow 2^{-\chi(N) / 2} l \text {. }
$$

(The identification of the determinant lines is defined by $R$ and by $d_{c}$ in the right column of (1.53).)

For an arbitrary nonzero $m \in \operatorname{Det}(M, Z, N)$ the following equality is deduced from (1.55) and from the commutativity of (1.53):

$$
\left(\varphi_{a n} T_{0}(M, Z)\right)(m)=2^{-\chi(N)}\left(\varphi_{c} T_{0}(M, Z)\right)(m) .
$$


Theorem 1.1 and Proposition 1.3 claim that

$$
\begin{aligned}
\varphi_{a n} T_{0}(M, Z) & =T_{0}\left(M_{1}, N \cup Z_{1}\right) \otimes T_{0}\left(M_{2}, N \cup Z_{2}\right) \otimes T_{0}(N), \\
\varphi_{c} \tau_{0}(X, V) & =\tau_{0}\left(X_{1}, W \cup V_{1}\right) \otimes \tau_{0}\left(X_{2}, W \cup V_{2}\right) \otimes \tau_{0}(W) .
\end{aligned}
$$

The isometries (1.56) and (1.57) involve the equality

$$
\begin{aligned}
& e(M, Z)=\log _{2}\left(T_{0}(M, Z) / \tau_{0}(X, V)\right)= \\
= & -\chi(N)+\left(\sum_{k=1,2} \log _{2}\left(T_{0}\left(M_{k}, N \cup Z_{k}\right) / \tau_{0}\left(X_{k}, W \cup V_{k}\right)\right)\right)+\log _{2}\left(T_{0}(N) / \tau_{0}(W)\right) .
\end{aligned}
$$

Thus the lemma is proved.

Let $\nu=(\alpha, \beta) \in \mathbb{R}^{2} \backslash(0,0)$ and let $\left(C^{\bullet}\left(X_{\nu}, V\right), d_{c}\right)$ be the complex of pairs of cochains $\left(c_{1}, c_{2}\right), c_{k} \in C^{\bullet}\left(X_{k}, V_{k}\right)$, with the $\nu$-transmission boundary condition (similar to (1.13)) on $W \subset \partial X_{k}$ between their geometrical restrictions

$$
\alpha i_{W, 1}^{*} c_{1}=\beta i_{W, 2}^{*} c_{2}
$$

The integration over the simplexes provides us with a quasi-isomorphism of the complexes:

$$
R_{\nu}:\left(D R^{\bullet}\left(M_{\nu}, Z\right), d\right) \rightarrow\left(C^{\bullet}\left(X_{\nu}, V\right), d_{c}\right)
$$

(i.e., $R_{\nu}$ induces an isomorphism between the corresponding cohomology).

The morphism of complexes $r_{\nu, c}:\left(C^{\bullet}\left(X_{\nu}, V\right), d_{c}\right) \rightarrow C^{\bullet}\left(W, d_{c}\right)$ is defined by analogy with the definition of $r_{\nu}$. Its value on each element $\left(c_{1}, c_{2}\right) \in C^{\bullet}\left(X_{\nu}, V\right)$ is

$$
r_{\nu, c}\left(c_{1}, c_{2}\right)=\left(\alpha^{2}+\beta^{2}\right)^{-1 / 2}\left(\beta i_{W, 1}^{*} c_{1}+\alpha i_{W, 2}^{*} c_{2}\right)
$$

The vertical arrows in the following diagram of complexes are quasi-isomorphisms ${ }^{9}$

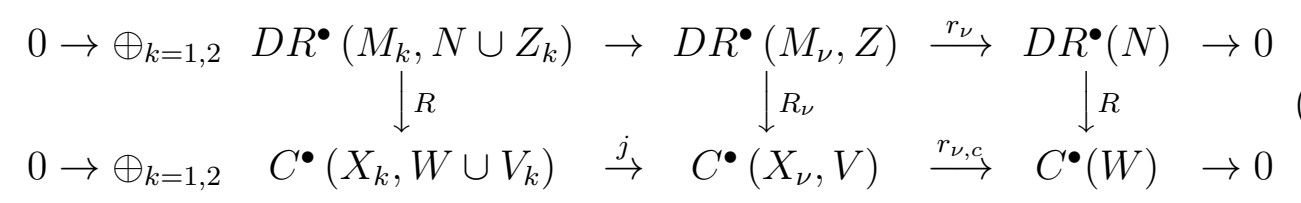

This diagram is commutative. The left horisontal arrows in it are the natural inclusions. Let $\varphi_{\nu}^{c}$ be the identification

$$
\varphi_{\nu}^{c}: \operatorname{det} H^{\bullet}\left(C\left(X_{\nu}, V\right)\right) \stackrel{R_{\nu}}{=} \operatorname{det} H^{\bullet}\left(M_{\nu}, Z\right) \rightarrow \operatorname{Det}(M, Z, N)
$$

defined by the bottom row of this diagram.

\footnotetext{
${ }^{9} R_{\nu}$ is a quasi-isomorphism according to Proposition 2.3.
} 
Remark 1.7. The equality

$$
\varphi_{\nu}^{c}=\varphi_{\nu}^{a n}
$$

follows from the commutativity of (1.59). But $\varphi_{1,1}^{c} \neq \varphi_{c}$ (in contrast with the identity $\left.\varphi_{1,1}^{a n}=\varphi_{a n}\right)$. According to (1.56) it holds that

$$
\varphi_{1,1}^{c}=\varphi_{a n}=2^{-\chi(N)} \varphi_{c}
$$

The space $C^{j}\left(X_{\nu}, V\right)$ is a subspace of $\oplus_{k=1,2} C^{j}\left(X_{k}, V_{k}\right)$. The Hilbert structure on $C^{j}\left(X_{k}, V_{k}\right)$ is defined by the orthonormal basises of cochains $\left\{\delta_{e}\right\}$ (parametrized by $j$-dimensional simplexes $e$ of $\left.X_{k} \backslash V_{k}\right)$. So the Hilbert structures on $C^{\bullet}\left(X_{\nu}, V\right)$ and on $\operatorname{det} C^{\bullet}\left(X_{\nu}, V\right)$ are defined. The scalar combinatorial torsion is defined as in (1.1):

$$
T\left(C^{\bullet}\left(X_{\nu}, V\right), d_{c}\right):=\exp \left(\left.\sum(-1)^{j} j \partial_{s} \zeta_{j, \nu}^{c}(s)\right|_{s=0}\right)
$$

where $\zeta_{j, \nu}^{c}(s):=\operatorname{Tr}^{\prime}\left(\left(\Delta_{j, \nu}^{c}\right)^{-s}\right)$ is the sum $\Sigma^{\prime} \lambda^{-s}$ over all the nonzero eigenvalues $\lambda$ of the finite-dimensional operator $\Delta_{j, \nu}^{c}=\left(d_{c}^{*} d_{c}+d_{c} d_{c}^{*} \mid C^{j}\left(X_{\nu}, V\right)\right)$ (with their multiplicities $), d_{c}^{*}$ is adjoint to $d_{c}$ in $C^{j}\left(X_{\nu}, V\right)$ with respect to the Hilbert structure in $C^{\bullet}\left(X_{\nu}, V\right)$.

The combinatorial torsion is the following norm on $\operatorname{det} H^{\bullet}\left(C\left(X_{\nu}, V\right)\right)^{10}$ :

$$
\tau_{0}\left(X_{\nu}, Z\right):=\|\cdot\|_{\operatorname{det} H \bullet\left(C\left(X_{\nu}, V\right)\right)}^{2} \cdot T\left(C^{\bullet}\left(X_{\nu}, V\right), d_{c}\right),
$$

where the norm on det $H^{\bullet}\left(C\left(X_{\nu}, V\right)\right)$ is defined by the Hilbert structures on the subquotions $H^{j}\left(C\left(X_{\nu}, V\right)\right)$ of the Hilbert spaces $C^{j}\left(X_{\nu}, V\right)$.

Remark 1.8. For each $\nu=(\alpha, \beta) \in \mathbb{R}^{2} \backslash(0,0)$ the combinatorial torsion $\tau_{0}\left(X_{\nu}, V\right)$ is an invariant of the combinatorial structure defined by a smooth triangulation of the triplet $[(M, \partial M) ; Z ; N]$, where $M$ is a manifold with a smooth boundary $\partial M, Z$ is a union of some connected components of $\partial M$, and $N$ is a smooth codimension one closed submanifold of $M$ with a trivial normal bundle $\left(\left.T M\right|_{N}\right) / T N$.

Proposition 1.5. The combinatorial torsion norm $\tau_{0}\left(X_{\nu}, Z\right)$ is isometric under the identification (1.60) to the tensor product of the combinatorial torsion norms:

$$
\varphi_{\nu}^{c} \tau_{0}\left(X_{\nu}, V\right)=\tau_{0}\left(X_{1}, W \cup V_{1}\right) \otimes \tau_{0}\left(X_{2}, W \cup V_{2}\right) \otimes \tau_{0}(W) .
$$

\footnotetext{
${ }^{10} \mathrm{It}$ is isomorphic to $\operatorname{det} H^{\bullet}\left(M_{\nu}, Z\right)$ under the quasi-isomorphism $R_{\nu}$.
} 
Proof. Under the identification (1.60), the Hilbert space $C^{\bullet}\left(X_{\nu}, V\right)$ is isometric to the tensor product of the Hilbert spaces on $\operatorname{det} C^{\bullet}\left(X_{k}, W \cup V_{k}\right)$ (for $\left.k=1,2\right)$ and on $\operatorname{det} C^{\bullet}(W)$. (The Hilbert structures on on $C^{\bullet}\left(X_{\nu}, V\right), C^{\bullet}\left(X_{k}, W \cup V_{k}\right)$, and on $C^{\bullet}(W)$ are defined above.) Indeed, let $\rho_{\nu, c}: C^{j}(W) \rightarrow C^{j}\left(X_{\nu}, V\right)$ be linear maps defined for $w \in C^{j}(W)$ by

$$
\rho_{\nu, c}(w)=\left(\alpha^{2}+\beta^{2}\right)^{-1 / 2}(\beta w, \alpha w) \in C^{j}\left(X_{\nu}, V\right)
$$

Then $r_{\nu, c} \rho_{\nu, c}=$ id on $C^{\bullet}(W), \rho_{\nu, c}$ is an isometry between $C^{j}(W)$ and $\operatorname{Im} \rho_{\nu, c}$, and $\operatorname{Im} \rho_{\nu, c}$ is the orthogonal complement in $C^{\bullet}\left(X_{\nu}, V\right)$ to the image of the natural inclusion $j: \oplus_{k=1,2} C^{\bullet}\left(X_{k}, W \cup V_{k}\right) \hookrightarrow C^{\bullet}\left(X_{\nu}, V\right)$ (where $j$ is an isometry onto $\operatorname{Im} j$ ). So the identification $\varphi_{\nu}^{c}$ is the isometry of the combinatorial torsion norms.

The number $e\left(M_{\nu}, Z\right) \in \mathbb{R}$ is defined as the logarithm of the ratio between the analytic and the combinatorial torsion norms:

$$
e\left(M_{\nu}, Z\right):=\log _{2}\left(T_{0}\left(M_{\nu}, Z\right) / \tau_{0}\left(X_{\nu}, V\right)\right) .
$$

Corollary 1.2. Under the conditions of Lemma 1.2, the equality holds:

$$
e\left(M_{\nu}, Z\right)=e\left(M_{1}, Z_{1} \cup N\right)+e\left(M_{2}, Z_{2} \cup N\right)+e(N),
$$

where $Z$ is a union of some connected components of $\partial M$ and $Z_{k}=Z \cap \partial M_{k}$.

Corollary 1.3. e $\left(M_{\nu}, Z\right)$ is independent of $\nu \in \mathbb{R}^{2} \backslash(0,0)$.

Corollary 1.4. For an arbitrary $\nu \in \mathbb{R}^{2} \backslash(0,0)$ the equality holds:

$$
e\left(M_{\nu}, Z\right)-e(M, Z)=\chi(N) \text {. }
$$

This equality follows from Lemma 1.4 and from (1.64).

Remark 1.9. Even for $\nu=(1,1)$ the number $e\left(M_{\nu}, Z\right)$ differs from $e(M, Z)$ in the case $\chi(N) \neq 0$.

1.4.2. Ratio of the analytic torsion norm and the combinatorial torsion norm for spheres and disks. Spherical Morse surgeries. The values of $e(M)$ and $e(M, \partial M)$, where $M$ is a sphere $S^{n}$ or a disk $D^{n}$ (with a direct product metric near $\partial D^{n}=S^{n-1}$ ) are deduced now from Lemma 1.4.

Lemma 1.5. 1. For all the spheres, e $\left(S^{n}\right)$ is zero.

2. For even-dimensional disks, $e\left(D^{2 n}\right)$ and $e\left(D^{2 n}, \partial D^{2 n}\right)$ are zero.

3. For all odd-dimensional disks, $e\left(D^{2 n+1}\right)$ and $e\left(D^{2 n+1}, \partial D^{2 n+1}\right)$ are equal to one. 
Proof. A closed interval $D^{1}$ is obtained by gluing two intervals $D^{1}=D^{1} \cup_{p t} D^{1}$ in their common boundary point. Lemma 1.4 claims in this case that

$$
e\left(D^{1}\right)=2 e\left(D^{1}, p t\right)+e(p t)-\chi(p t)
$$

Since $e(p t)=0$, we see that (1.52) involves the equalities

$$
e\left(D^{1}\right)=e\left(D^{1}, p t\right)=e\left(D^{1}, \partial D^{1}\right)
$$

Hence (1.66) involves $e\left(D^{1}\right)=\chi(p t)=1$.

A circle $S^{1}$ is obtained by gluing two intervals, namely $S^{1}=D^{1} \cup_{\partial D^{1}} D^{1}$. So, according to Lemma 1.4 and to (1.67), we have

$$
e\left(S^{1}\right)=2 e\left(D^{1}\right)+e\left(\partial D^{1}\right)-\chi\left(\partial D^{1}\right)=0 .
$$

Suppose (by the induction hypothesis) that $e\left(S^{m}\right)=0$ for $m \leq n-1$. The sphere $S^{n}$ (for $\left.n \geq 2\right)$ is the union $\left(D^{n-1} \times S^{1}\right) \bigcup_{S^{n-2} \times S^{1}}\left(D^{2} \times S^{n-2}\right)=S^{n}$ Indeed, $S^{n}=$ $\left\{\left(x_{1}, \ldots, x_{n+1}\right) \in \mathbb{R}^{n+1}: \sum x_{j}^{2}=1\right\}$, the disk $D^{2}$ in $D^{2} \times S^{n-2}$ in the decomposition above corresponds to $\left\{\left(x_{1}, x_{2}\right): x_{1}^{2}+x_{2}^{2} \leq \varepsilon\right\}$ and $S^{n-2}=\left\{\left(x_{j}\right) \in S^{n}, x_{1}=x_{2}=0\right\}$. Lemma 1.4 claims in this case that (since $\chi\left(S^{n-2} \times S^{1}\right)=0$ )

$$
e\left(S^{n}\right)=e\left(D^{n-1} \times S^{1}, S^{n-2} \times S^{1}\right)+e\left(D^{2} \times S^{n-2}, S^{1} \times S^{n-2}\right)+e\left(S^{n-2} \times S^{1}\right) .
$$

The equalities below are deduced from the induction hypothesis, from Lemma 1.3 $((1.52),(1.51))$, and from (1.68):

$$
\begin{aligned}
e\left(D^{n-1} \times S^{1}, S^{n-2} \times S^{1}\right) & =e\left(D^{n-1} \times S^{1}\right)-e\left(S^{n-2} \times S^{1}\right), \\
e\left(D^{2} \times S^{n-2}, S^{1} \times S^{n-2}\right) & =e\left(D^{2} \times S^{n-2}\right)-e\left(S^{1} \times S^{n-2}\right) \\
e\left(S^{1} \times S^{n-2}\right)=0, & e\left(D^{n-1} \times S^{1}\right)=0 \\
e\left(D^{2} \times S^{n-2}\right) & =\chi\left(S^{n-2}\right) e\left(D^{2}\right) .
\end{aligned}
$$

Hence the combinatorial torsion norm is equal to the analytic torsion norm for all odd-dimensional spheres $S^{2 m+1}$ :

$$
e\left(S^{2 m+1}\right)=0, \quad T_{0}\left(S^{2 m+1}\right)=\tau_{0}\left(S^{2 m+1}\right) .
$$

It follows from Lemma 1.3 and from (1.68) that $e\left(D^{2}\right)=e\left(D^{2}, \partial D^{2}\right)$. It is deduced from Lemma 1.4 and from (1.68) that $e\left(S^{2}\right)=2 e\left(D^{2}\right)$. According to (1.69) the equality $e\left(S^{2 m}\right)=0$ for all even-dimensional spheres is a consequence of the equality $e\left(S^{2}\right)=0$. 
Let $\left(M, g_{M}\right)$ be any closed Riemannian manifold of even dimension $2 n$. Then the scalar analytic torsion $T\left(M, g_{M}\right)$ is equal to 1 . (This equality was proved in [RS], Theorem 2.1, with the help of the equality

$$
\sum(-1)^{j} j m(\lambda, j)=0,
$$

where $\lambda$ is an arbitrary nonzero eigenvalue of $\Delta_{j}$ on $D R^{j}(M)$ and $m(\lambda, j)$ is its multiplicity. The latter assertion follows from the symmetry relation $m(\lambda, j)=$ $m(\lambda, 2 n-j)$, which is obtained applying the operator $*$ for a Riemannian metric $g_{M}$ to the $\lambda$-eigenforms for $\Delta_{j}$.) So (in particular) the torsion norm $T_{0}\left(S^{2}\right)$ is equal to $\|\cdot\|_{\operatorname{det} H \bullet\left(S^{2}\right)}^{2}$, where the norm on $H^{\bullet}\left(S^{2}\right)$ is the norm defined by $g_{M}$ on the harmonic forms Ker $\Delta^{\bullet}$. (The unduced norm $\|\cdot\|_{\operatorname{det} H \bullet\left(S^{2}\right)}^{2}$ does not depend on the metric $g_{S^{2}}$, as it follows from the invariance of $T_{0}\left(M, g_{M}\right)$ with respect to $g_{M}$, proved above.)

Let $v$ be a volume of $S^{2}$ relative to a Riemannian metric $g_{S^{2}}$. Then the element $h \in \operatorname{det} H^{\bullet}\left(S^{2}\right)$ defined below is of the norm 1 :

$$
h=\left(v^{-1 / 2} \cdot 1_{S^{2}}\right)^{-1} \otimes\left(v^{-1 / 2}\left(* 1_{S^{2}}\right)\right)^{-1}, \quad\|h\|_{\operatorname{det} H \bullet\left(S^{2}\right)}^{2}=1
$$

(here $1_{S^{2}}$ is the constant $1 \in D R^{0}\left(S^{2}\right)$ and $* 1_{S^{2}}$ is the $g_{S^{2}}$-volume form).

The sphere $S^{2}$ has a cell decomposition ${ }^{11} X_{S^{2}}: X:=D^{2} \cup_{\partial D^{2}} p t$. Hence the element $h_{c} \in \operatorname{det} C^{\bullet}\left(X_{S^{2}}\right)$ defined below is of the norm 1 :

$$
h_{c}=\left(\delta_{p t}\right)^{-1} \otimes\left(\delta_{D^{2}}\right)^{-1},\left\|h_{c}\right\|_{\operatorname{det} C}^{2} \cdot\left(X_{S^{2}}\right)=1 .
$$

(For this cell-decomposition $d_{c}=0$, and so $\operatorname{det} C^{\bullet}\left(X_{S^{2}}\right)$ is the same as $\operatorname{det} H^{\bullet}\left(S^{2}\right)$ without the $d_{c}$-identification. The cochains $\delta_{p t}, \delta_{D^{2}}$ are the basic elements in $H^{0}\left(S^{2}\right)$, $H^{2}\left(S^{2}\right)$.)

The integration homomorphism $R: D R^{\bullet}\left(S^{2}\right) \rightarrow C^{\bullet}\left(X_{S^{2}}\right)$ maps $1_{S^{2}}$ to $\delta_{p t}$ and $* 1_{S^{2}}$ to $v \cdot \delta_{D^{2}}$. So $R(h)=h_{c}$ and we have

$$
e\left(S^{2}\right)=0, \quad e\left(S^{2 m}\right)=0 .
$$

The equalities below follow from Lemmas 1.3, 1.4, and from (1.70), (1.71):

$$
\begin{aligned}
0=e\left(S^{n}\right) & =2 e\left(D^{n}, \partial D^{n}\right)+e\left(S^{n-1}\right)-\chi\left(S^{n-1}\right) \\
e\left(D^{n}, \partial D^{n}\right) & =e\left(D^{n}\right)-e\left(S^{n-1}\right)=e\left(D^{n}\right) \\
e\left(D^{n}, \partial D^{n}\right) & =2^{-1} \chi\left(S^{n-1}\right)=e\left(D^{n}\right) .
\end{aligned}
$$

Lemma 1.5 is proved.

\footnotetext{
${ }^{11}$ This $C W$-complex (cell stratification) has a subdivision which is a $C^{1}$-triangulation of $S^{2}$. So as the combinatorial torsion is defined also for $C W$-complexes and as it is invariant under subdivisions, $\tau\left(S^{2}\right)$ can be computed from this cell stratification ([Mi], Sections 7, 8, 12.3).
} 
The equality $e\left(D^{m+1} \times S^{n}\right)=e\left(D^{m+1} \times S^{n}, \partial\left(D^{m+1} \times S^{n}\right)\right)$ holds by Lemmas 1.3 and 1.5.

Corollary 1.5. For arbitrary $n, m \geq 0$ the equality holds:

$$
e\left(D^{m+1} \times S^{n}\right)=e\left(S^{m} \times D^{n+1}\right) .
$$

(According to Lemma 1.5, each side of (1.72) is equal to 2 in the case of a pair of even numbers $(m, n)$ and it is equal to zero for other pairs $(m, n)$.)

Let $M$ be a compact manifold with a smooth boundary $\partial M$ and let $Z$ be a union of some connected components of $\partial M$. Let $\widetilde{M}$ be obtained by some spherical Morse surgery (with a trivial normal bundle) of $M$ (i.e., there exists a manifold $\left(M_{1}, \partial M_{1}\right) \subset$ $M \backslash \partial M, M_{1} \simeq D^{m+1} \times S^{n}, m+n+1=\operatorname{dim} M$, with $\partial M_{1}=S^{m} \times S^{n}, M=M_{1} \cup_{\partial M} M_{2}$, such that $\widetilde{M}=\widetilde{M}_{1} \cup_{\partial \widetilde{M}_{1}} M_{2}$ is obtained by gluing $\widetilde{M}_{1}=S^{m} \times D^{n+1}$ and $M_{2}$ by a diffeomorphism $f: \partial \widetilde{M}_{1} \sim \partial M_{2}$ ).

Let the metrics $g_{M}$ and $g_{\widetilde{M}}$ be direct product metrics near $\partial M$ and $\partial \widetilde{M}$. (It is proved above that the numbers $e(M, Z)$ and $e(\widetilde{M}, Z)$ do not depend on the metrics $g_{M}, g_{\widetilde{M}}$ supposed to be direct product metrics near $\partial M$ and near $\partial \widetilde{M}$.)

Lemma 1.6. The number $e(M, Z)$ is invariant under the spherical Morse surgeries with a trivial normal bundle, i.e., the equality holds

$$
e(M, Z)=e(\widetilde{M}, Z)
$$

Proof. The metrics $g_{M}$ and $g_{\widetilde{M}}$ can be replaced by Riemannian metrics on $M$ and $\widetilde{M}$ which are direct product metrics on $\partial M_{1} \times I$ and $\partial \widetilde{M}_{1} \times I$ near $\partial M_{1} \subset M$ and near $\partial \widetilde{M}_{1} \subset \widetilde{M}$ (and which are direct product metrics near $\partial M$ and $\left.\partial \widetilde{M}\right)$. Lemma 1.4 claims in this case that

$$
\begin{aligned}
& e(M, Z)=e\left(M_{1}, \partial M_{1}\right)+e\left(M_{2}, \partial M_{1} \cup Z\right)+e\left(\partial M_{1}\right)-\chi\left(\partial M_{1}\right), \\
& e(\widetilde{M}, Z)=e\left(\widetilde{M}_{1}, \partial \widetilde{M}_{1}\right)+e\left(M_{2}, \partial \widetilde{M}_{1} \cup Z\right)+e\left(\partial \widetilde{M}_{1}\right)-\chi\left(\partial \widetilde{M}_{1}\right) .
\end{aligned}
$$

The smooth closed manifolds $\partial \widetilde{M}_{1}$ and $\partial M_{1}$ are diffeomorphic. Hence

$$
e\left(\partial M_{1}\right)=e\left(\partial \widetilde{M}_{1}\right), \quad \chi\left(\partial M_{1}\right)=\chi\left(\partial \widetilde{M}_{1}\right), \quad e\left(M_{2}, \partial M_{1} \cup Z\right)=e\left(M_{2}, \partial \widetilde{M}_{1} \cup Z\right) .
$$

Corollary 1.5 and Lemmas 1.3 and 1.5 claim that $e\left(M_{1}, \partial M_{1}\right)=e\left(\widetilde{M}_{1}, \partial \widetilde{M}_{1}\right)$. So the equality (1.73) follows from (1.74). 


\subsubsection{Proof of the generalized Ray-Singer conjecture.}

Theorem 1.3 (Classical Ray-Singer conjecture). For any closed Riemannian manifold $\left(M, g_{M}\right)$ its analytic torsion norm is equal to the combinatorial torsion norm

$$
T_{0}(M)=\tau_{0}(M)
$$

Proof. There is a smooth Morse function $f$ on a direct product $M \times I$ (i.e., a function with the nondegenerate isolated critical points with different critical values) such that the following holds. Its minimum value is equal to zero, $f^{-1}(0)=M \times \partial I$, and the zero is not a critical value of $f$. Its maximum value $\max _{M \times I} f$ equals 1 and the maximum value level is the only one point. Namely $f^{-1}(1)$ is an interior point of $M \times(I, \partial I)$.

As $f^{-1}(1-\varepsilon)$ (where $\varepsilon>0$ is very small) is a sphere $S^{n}(n=\operatorname{dim} M)$, there exists a sequence of spherical Morse surgeries (given by transformations of levels $f^{-1}(x), x \in(0,1-\varepsilon)$ for $x$ divided by critical values) such that their composition is a transformation of a manifold ${ }^{12} M \cup M=M \times \partial I=f^{-1}(0)$ into $S^{n}=f^{-1}(1-\varepsilon)$.

Aa a consequence of Lemma 1.6 in this case we get

$$
2 e(M)=e(M \cup M)=e\left(S^{n}\right) .
$$

Lemma 1.5 claims that $0=e\left(S^{n}\right)=e(M)$. Thus, the Ray-Singer conjecture is proved.

Let $\left(M, g_{M}\right)$ be a compact Riemannian manifold with a smooth boundary $\partial M$. Let $Z$ be a union of some connected components of $\partial M$ and let $g_{M}$ be a direct product metric near $\partial M$. The following two theorems are generalizations of the Ray-Singer conjecture.

Theorem 1.4. Under the conditions above, the following equality holds for a manifold with a smooth boundary:

$$
T_{0}(M, Z)=2^{\chi(\partial M) / 2} \tau_{0}(M, Z) .
$$

Proof. Lemma 1.3 claims that $e(M, Z)=e(M, \partial M)+e(\partial M \backslash Z)$. According to Theorem 1.3, $e(\partial M \backslash Z)$ is equal to zero. Hence $e(M, Z)=e(M, \partial M)$. In the case of $\partial M \neq \emptyset$ there is a mirror-symmetric closed Riemannian manifold $P=M \cup_{\partial M} M$ obtained by gluing two copies of $\left(M, g_{M}\right)$ along $\partial M$. According to Lemma 1.4, we have

$$
e(P)=2 e(M, \partial M)+e(\partial M)-\chi(\partial M)
$$

\footnotetext{
${ }^{12}$ The manifold $M$ is not supposed to be orientable.
} 
Theorem 1.3 claims that $e(\partial M)=0=e(P)$. Thus, we get

$$
e(M, Z)=e(M, \partial M)=2^{-1} \chi(\partial M),
$$

which is equivalent to $(1.75)$.

Let $\left(M, Z, g_{M}\right)$ be as in Theorem 1.4. Let $N$ be a codimension one in $M$ twosided in $M$ closed submanifold $N \subset M \backslash \partial M$. Let $M$ be obtained by gluing $M_{1}$ and $M_{2}$ along $N$. Let $g_{M}$ be a direct product metric near $N$ and let the $\nu$-transmission boundary conditions (1.13) be given on $N$ (where $\nu=(\alpha, \beta) \in \mathbb{R}^{2} \backslash(0,0)$ ).

Theorem 1.5. The analytic torsion norm is expressed by the combinatorial torsion norm (in the case of the $\nu$-transmission interior boundary condition on $N$ ) as follows

$$
T_{0}\left(M_{\nu}, Z\right)=2^{\chi(\partial M) / 2+\chi(N)} \tau_{0}\left(M_{\nu}, Z\right) .
$$

Proof. The equality (1.65) claims that $e\left(M_{\nu}, Z\right)=e(M, Z)+\chi(N)$. So the assertion of the theorem follows from Theorem 1.4 and from the equality (1.65).

Remark 1.10. This proof of the generalizations of the Ray-Singer conjecture does not use any explicit expressions for the scalar analytic torsions of any special classes of manifolds. The proofs in [Mü1], [Ch] of the classical Ray-Singer conjecture essentially used the explicit expressions for the scalar analytic torsions for spheres and lens spaces. (The latter expressions were obtained by D.B. Ray in [Ra]. He computed there the scalar analytic torsion for lens spaces and spheres with homogeneous metrics by explicit calculations of the $\zeta$-functions for the corresponding Laplacians using Gegenbauer's polynomials.) The proof in [Mü1] used the precise estimates of [DP] for the eigenvalues of the corresponding combinatorial Laplacians. The proof of [Ch] used the Lerch formula for the derivative at zero of the zeta-function of Riemann ([WW], 13.21, 12.32). We don't use this formula. (Its new proof is obtained here.) Our proof of the generalized Ray-Singer formula is based on a gluing property for the analytic torsion norms. This property is proved here for a general gluing two Riemannian manifolds by a diffeomorphism of some connected components of their boundaries. It is proved without any computations of asymptotics of eigenvalues and eigenforms for the corresponding Laplacians.

2. Gluing formula for analytic torsion norms. Proof of Theorem 1.1

2.1. Strategy of the proof. In Section 1 the generalized Ray-Singer conjecture for a manifold with a smooth boundary is deduced from Theorem 1.1. Namely it is deduced from the gluing formula

$$
\varphi_{a n} T_{0}(M, Z)=T_{0}\left(M_{1}, Z_{1} \cup N\right) \otimes T_{0}\left(M_{2}, Z_{2} \cup N\right) \otimes T_{0}(N),
$$

which holds under the conditions of Theorem 1.1 (where $Z_{k}:=Z \cap \partial M_{k}$ ). The identification $\varphi_{a n}$ in (2.1) is defined in (1.9) with the help of the exact sequence 
(1.10) of the de Rham complexes. It is proved in Lemma 1.2 that the equality (2.1) follows from the assertion that under the conditions of Theorem 1.1, the induced analytic torsion norm ${ }^{13} \varphi_{\nu}^{a n} T_{0}\left(M_{\nu}\right)$ does not depend on a parameter $\nu$ of the interior boundary conditions. The latter statement means that the equality

$$
\varphi_{\nu}^{a n} T_{0}\left(M_{\nu}, Z\right)=c_{0} T_{0}\left(M_{1}, Z_{1} \cup N\right) \otimes T_{0}\left(M_{2}, Z_{2} \cup N\right) \otimes T_{0}(N),
$$

holds with a positive constant $c_{0}$ which is independent of $\nu \in \mathbb{R}^{2} \backslash(0,0)$. (However, it is not supposed in Lemma 1.2 that $c_{0}$ is independent of $M, N, g_{M}$, and $Z$.)

The strategy of the proof of the equality $(2.2)$ is as follows. First we prove that $c_{0}$ is constant on each of four connected components

$$
U_{j} \subset U:=\left\{(\alpha, \beta) \in \mathbb{R}^{2}: \alpha \beta \neq 0\right\} .
$$

Then it is enough to prove that $c_{0}(\nu)$ is continuous as a function of $\nu$ for $\nu \in$ $\mathbb{R}^{2} \backslash(0,0)$. These two assertions provide us with a proof of the equality $(2.2)$.

Let $\nu_{0} \in U$ and let $a>0$ be a number not belonging to the spectrum $S\left(\nu_{0}\right):=$ $\bigcup_{i}$ Spec $\Delta_{i}\left(M_{\nu_{0}}, g_{M}\right) \subset \mathbb{R}_{+}$of the Laplacians on $D R^{\bullet}\left(M_{\nu}, Z\right)$. This spectrum is discrete according to Theorem 3.1. In particular, each its eigenvalue is of a finite multiplicity. Let $W_{a}^{i}(\nu)$ be a subspace of $D R^{i}\left(M_{\nu}, Z\right)$, spanned by all the eigenforms $\omega_{\lambda}$ for $\Delta_{\nu, i}:=\Delta_{i}\left(M_{\nu}, g_{M}\right)$ with their eigenvalues $\lambda \leq a$. Then $d W_{a}^{i}(\nu) \subset W_{a}^{i+1}(\nu)$. So $\left(W_{a}^{\bullet}(\nu), d\right)$ is a finite-dimensional subcomplex of $\left(D R^{\bullet}\left(M_{\nu}, Z\right), d\right)$ equiped with the natural Hilbert structures on $W_{a}^{\bullet}(\nu) \hookrightarrow D R^{\bullet}\left(M_{\nu}, Z\right)$ (defined by $\left.g_{M}\right)$.

Let $\|\cdot\|_{\operatorname{det} W_{a}^{\bullet}(\nu)}^{2}$ be the induced norm on $\operatorname{det} W_{a}^{\bullet}(\nu)$. For $\nu$ very close to $\nu_{0}$ it holds also that $a \notin S(\nu)$ (Proposition 3.1). By the definition of $W_{a}^{\bullet}(\nu)$, its cohomology $H^{j}\left(W_{a}^{\bullet}(\nu)\right)$ are canonically identified with the space of harmonic forms $\operatorname{Ker} \Delta_{j}\left(M_{\nu}, g_{M}\right)$. The differential $d$ in $W_{a}^{\bullet}(\nu)$ induces the identification

$$
d_{W}: \operatorname{det} W_{a}^{\bullet}(\nu) \rightarrow \operatorname{det} \operatorname{Ker} \Delta_{\bullet}\left(M_{\nu}, g_{M}\right) .
$$

According to Lemma 1.1 there is a canonical identification between the harmonic forms and the cohomology of the de Rham complex (the latter one is independent of $\left.g_{M}\right)$ :

$$
\operatorname{Ker} \Delta_{i}\left(M_{\nu}, g_{M}\right)=H^{i}\left(D R\left(M_{\nu}, Z\right)\right) .
$$

So there is the induced canonical identification of the determinant lines:

$$
\operatorname{det} \operatorname{Ker} \Delta_{\bullet}\left(M_{\nu}, g_{M}\right)=\operatorname{det} H^{\bullet}\left(D R\left(M_{\nu}, Z\right), d\right) .
$$

Let $\|\cdot\|_{\operatorname{det} H \bullet\left(M_{\nu}\right)}^{2}$ be a norm on $\operatorname{det} H^{\bullet}\left(M_{\nu}, Z\right):=\operatorname{det} H^{\bullet}\left(D R\left(M_{\nu}, Z\right), d\right)$ induced by the identifications (2.5) and (2.6) from the Hilbert structure on the harmonic forms $\operatorname{Ker} \Delta_{\bullet}\left(M_{\nu}, g_{M}\right)$. (This structure is defined by the Riemannian metric $g_{M}$.)

${ }^{13}$ The identification $\varphi_{\nu}^{a n}$ is defined by the short exact sequence (1.14). 
The identification (2.4) is not an isometry of the norms $\|\cdot\|_{\operatorname{det} H \bullet\left(M_{\nu}\right)}^{2}$ and $\|\cdot\|_{\operatorname{det} W_{a}^{\bullet}(\nu)}^{2}$ in general. The norm $\|\cdot\|_{\operatorname{det} H \bullet\left(M_{\nu}\right)}^{2}$ has to be multiplied by an additional factor for the identification (2.4) to become an isometry. This factor is the scalar analytic torsion of a complex $\left(W_{a}^{\bullet}(\nu), d\right)$, defined by the general formula (1.1). We can conclude that the analytic torsion norm $T_{0}\left(M_{\nu}, Z\right)$ on the determinant of $H^{\bullet}\left(D R\left(M_{\nu}, Z\right)\right)$ is isometric (under the identifications (2.4) and (2.6)) to the norm

$$
T_{0}\left(M_{\nu}, Z ; a\right):=\|\cdot\|_{\operatorname{det} W_{a}^{*}(\nu)}^{2} \exp \left(\left.\sum(-1)^{j} j \partial_{s} \zeta_{\nu, j}(s ; a)\right|_{s=0}\right) .
$$

The zeta-function $\zeta_{\nu, j}(s ; a)$ is defined for $\operatorname{Re} s>(\operatorname{dim} M) / 2$ by the series $\sum_{\lambda>0} \lambda^{-s}$, where the sum is over all the eigenvalues $\lambda$ of $\Delta_{j}\left(\left(M_{\nu}, g_{M}\right)\right.$ ) (including their multiplicities), such that $\lambda>a$. This $\zeta$-function can be continued meromorphically to the whole complex plane $\mathbb{C}$ and it is regular at zero. The latter assertion follows from Theorem 3.1 and from the equality (which is obvious for $\operatorname{Re} s>(\operatorname{dim} M) / 2$ ):

$$
\zeta_{\nu, j}(s ; a)=\zeta_{\nu, j}(s)-\sum_{0<\lambda \leq a} \lambda^{-s}
$$

(The series for $\zeta_{\nu, j}(s), \operatorname{Re} s>(\operatorname{dim} M) / 2$, is the sum over all the nonzero eigenvalues of $\Delta_{j}\left(M_{\nu}, g_{M}\right)$ with their multiplicities, where $\lambda^{-s}:=\exp (-s \log \lambda)$ and $\log \lambda \in \mathbb{R}$ for $\lambda>0)$.

The identifications $d_{W}(2.4)$ and $\varphi_{a n}^{\nu}$ (the latter one is defined with the help of (1.14)) provide us (under the conditions of Lemma 1.2) with the identification:

$$
\varphi_{\nu}^{a n}(a): \operatorname{det} W_{a}^{\bullet}(\nu) \sim \operatorname{Det}(M, N, Z)
$$

$\left(\operatorname{Det}(M, N, Z)^{14}\right.$ is defined in (1.9)). The assertion that $c_{0}(\nu)$ is independent of $\nu$ on each connected component $U_{j}$ of $U(2.3)$ is equivalent to the following one. The analytic torsion norm $T_{0}\left(M_{\nu}, Z ; a\right)$ is transformed (under the identification (2.9)) into the norm on $\operatorname{Det}(M, N, Z)$ :

$$
\varphi_{\nu}^{a n}(a) \circ T_{0}\left(M_{\nu}, Z ; a\right)=c_{0}(\nu) T_{0}\left(M_{1}, Z_{1} \cup N\right) \otimes T_{0}\left(M_{2}, Z_{2} \cup N\right) \otimes T_{0}(N),
$$

where $c_{0}(\nu)$ is constant on each connected component $U_{j}$.

The action of $\varphi_{a n}^{\nu}(a)$ is as follows (by its definition):

$$
\varphi_{a n}^{\nu}(a) T_{0}\left(M_{\nu}, Z ; a\right)=T\left(M_{\nu}, Z ; a\right) \varphi_{a n}^{\nu}(a) \circ\|\cdot\|_{\operatorname{det} W_{a}^{\bullet}(\nu)}^{2},
$$

where the scalar analytic torsion $T\left(M_{\nu}, Z ; a\right)$ is defined as the scalar factor in $(2.7)$ :

$$
T\left(M_{\nu}, Z ; a\right):=\exp \left(\left.\sum(-1)^{j} j \partial_{s} \zeta_{\nu, j}(s ; a)\right|_{s=0}\right) \text {. }
$$

Let $\nu(\gamma), \gamma \in(\varepsilon, \varepsilon) \subset \mathbb{R}$, be a smooth curve in $U(2.3)$ and let $\nu(0)=\nu_{0}$. Let $\Pi_{j}\left(\nu_{0} ; a\right)$ be an ortogonal projection operator from $\left(D R^{j}(M)\right)_{2}$ onto $W_{a}^{j}\left(\nu_{0}\right)$ (relative

\footnotetext{
${ }^{14}$ To remind, $Z$ is the union of the connected components of $\partial M$, where the Dirichlet boundary conditions are given. The Neumann boundary conditions are given on $\partial M \backslash Z$.
} 
to the natural Hilbert structure $(1.23)$ in $\left.\left(D R^{j}(M)\right)_{2}\right)$. Let $p_{1}$ be a linear operator in $\left(D R^{\bullet}(M)\right)_{2}$, mapping $\left(\omega_{1}, \omega_{2}\right) \in\left(D R^{\bullet}(M)\right)_{2}$ to $\left(\omega_{1}, 0\right)$. (Respectively $p_{2}$ maps $\left(\omega_{1}, \omega_{2}\right)$ to $\left.\left(0, \omega_{2}\right).\right)$

Let $\nu$ and $\nu_{0}$ be arbitrary points from $U$. Then the following isomorphism of the de Rham complexes is defined (where $k_{\nu}:=\alpha / \beta$ for $\left.\nu=(\alpha, \beta) \in U\right)$ :

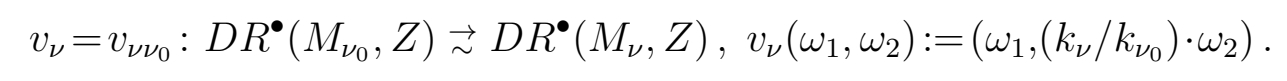

Thus the induced isomorphism is defined:

$$
v_{\nu *}: H^{\bullet}\left(D R\left(M_{\nu_{0}}, Z\right)\right) \rightarrow H^{\bullet}\left(D R\left(M_{\nu}, Z\right)\right)
$$

Let $a$ be a positive number from $\mathbb{R}_{+} \backslash S\left(\nu_{0}\right)$. Then for $\nu$ very close to $\nu_{0}$ the number $a$ is also from $\mathbb{R}_{+} \backslash S(\nu)$ (Proposition 3.1). The complexes $W_{a}^{\bullet}(\nu)$ and $W_{a}^{\bullet}\left(\nu_{0}\right)$ are isomorphic as abstract finite-dimensional complexes (and (2.12) provides us with a natural but not canonical isomorphism of these complexes). We have to compute the action of $\varphi_{a n}^{\nu}$ on the norms $\|\cdot\|_{\operatorname{det} W_{\boldsymbol{a}}(\nu)}^{2}$ for $\nu$ very close to $\nu_{0}$. However $\|\cdot\|_{W_{\boldsymbol{a}}(\nu)}^{2}$ are the norms on different complexes. So it is necessary to define some isomorphism between $W_{a}^{\bullet}\left(\nu_{0}\right)$ and $W_{a}^{\bullet}(\nu)$ and then to compute its action on $\|\cdot\|_{\operatorname{det} W_{a}^{\bullet}\left(\nu_{0}\right)}^{2}$ and on the space $\operatorname{Det}(M, N, Z)$. The choice (2.13) of such an identification is done below.

For $\nu$ very close to $\nu_{0}$ the subspaces $W_{a}^{\bullet}(\nu)$ and $W_{a}^{\bullet}\left(\nu_{0}\right)$ are very close in the $L_{2^{-}}$ completion $\left(D R^{\bullet}(M)\right)_{2}$ of $D R^{\bullet}\left(M_{\nu}, Z\right)=: D R^{\bullet}(\nu)$, according to Proposition 3.1. So the following isomorphism of these finite-dimensional complexes is well-defined:

$$
\begin{aligned}
& g_{\nu}=\Pi^{\bullet}(\nu ; a) \cdot v_{\nu \nu_{0}} \cdot j_{\nu_{0}}: \\
& W_{a}^{\bullet}\left(\nu_{0}\right) \hookrightarrow\left(D R^{\bullet}\left(\nu_{0}\right), d\right) \stackrel{v_{\nu \nu_{0}}}{\longrightarrow}\left(D R^{\bullet}(\nu), d\right) \underset{\Pi \bullet(\nu ; a)}{\longrightarrow}\left(W_{a}^{\bullet}(\nu), d\right),
\end{aligned}
$$

where $j_{\nu_{0}}$ is the natural inclusion of $W_{a}^{\bullet}\left(\nu_{0}\right)$ and $\Pi^{\bullet}(\nu ; a)$ is the orthogonal projection operator onto $W_{a}^{\bullet}(\nu)$. Its action on the norm $\|\cdot\|_{\operatorname{det} W_{a}^{\bullet}\left(\nu_{0}\right)}^{2}$ is computed by the following lemma.

Lemma 2.1. Let $l$ be an arbitrary nonzero element of $\operatorname{det} W_{a}^{\bullet}\left(\nu_{0}\right)$. Then the equality holds for any smooth variation $\nu(\gamma)$ of $\nu_{0}=\nu(0)$ :

$$
\left.\partial_{\gamma} \log \left\|g_{\nu} l\right\|_{\operatorname{det} W_{a}^{\bullet}(\nu)}^{2}\right|_{\gamma=0}=-\left.2 \partial_{\gamma} \log \left(k_{\nu}\right)\right|_{\gamma=0}\left(\sum(-1)^{j} \operatorname{Tr}\left(p_{2} \Pi^{j}\left(\nu_{0} ; a\right)\right)\right) .
$$

In (2.14) the rank (i.e., the dimension of the image) of the operator $p_{2} \Pi^{j}\left(\nu_{0} ; a\right)$ is less or equal to $\operatorname{dim} W_{a}^{\bullet}\left(\nu_{0}\right)$. This operator acts in $\left(D R^{\bullet}(M)\right)_{2}$.

Then the following lemma provides us with the variation formula for $T\left(M_{\nu}, Z ; a\right)$. 
Lemma 2.2. For $\gamma=0$ the equality holds:

$$
\partial_{\gamma} \log T\left(M_{\nu}, Z ; a\right)=\left.2 \partial_{\gamma} \log \left(k_{\nu}\right)\right|_{\gamma=0} \sum(-1)^{j} b_{1, j}\left(M_{\nu_{0}}, Z ; a\right)
$$

where $k_{\nu}:=\alpha / \beta$ for $\nu=(\alpha, \beta) \in U$. Here $b_{1, j}\left(M_{\nu_{0}}, Z ; a\right)$ is a constant coefficient (i.e., $t^{0}$-coefficient $q_{0}$ ) in the asymptotic expansion as $t \rightarrow+0$ of the trace of the operator below (acting in $\left.\left(D R^{j}(M)\right)_{2}\right)$ :

$\operatorname{Tr}\left(p_{1}\left\{\exp \left(-t \Delta_{\nu_{0}, j}\right)\left(1-\Pi^{j}\left(\nu_{0} ; a\right)\right)\right\}\right) \sim q_{-n} t^{-n / 2}+q_{-n+1} t^{-(n-1) / 2}+\ldots+q_{0} t^{0}+\ldots$

Remark 2.1. The operators $\exp \left(-t \Delta_{\nu_{0}, j}\right)$ and $\Pi^{j}\left(\nu_{0} ; a\right)$ acting in the $L_{2}$-completion $\left(D R^{j}(M)\right)_{2}$ of $D R^{j}\left(M_{\nu_{0}}, Z\right)$ (which coincides with the $L_{2}$-completion of $D R^{j}(M)$ ) have their images in the domain of definition of the Laplacian $D\left(\Delta_{\nu_{0}, j}\right) \subset D R^{j}\left(M_{\nu_{0}}, Z\right)$. The existence of the asymptotic expansion (2.15) follows from Theorem 3.2. The coefficients $q_{m}$ with $m \leq-1$ in (2.15) are independent of $a$. The coefficients $\tilde{q}_{m}$ of the asymptotic expansion for $\operatorname{Tr}\left(p_{1} \exp \left(-t \Delta_{\nu_{0}, j}\right)\right)$ are equal to the sums of the integrals over $M_{1}$ and over $\partial M_{1} \supset N$ of the locally defined densities on $M_{1}$ and on $\partial M_{1}$ (by Theorem 3.2). However, in the general case we cannot represent $\operatorname{Tr}\left(p_{1} \Pi^{j}\left(\nu_{0} ; a\right)\right)$ as an integral of a locally defined density (because there are no universal local formulas for the eigenforms $\omega_{\lambda}$ of $\Delta_{\nu_{0}, j}$ ). Hence there is no universal local formula for a coefficient $q_{0}$ in (2.15) but there are such formulas for $q_{m}=\tilde{q}_{m}$ with $m<0$.

Corollary 2.1. For an arbitrary nonzero $l \in \operatorname{det} W_{a}^{\bullet}\left(\nu_{0}\right)$ the equality holds

$$
\partial_{\gamma} \log \left\|g_{\nu} l\right\|_{T_{0}\left(M_{\nu}, Z\right)}^{2}=\left.2 \partial_{\gamma}\left(\log k_{\nu}\right)\right|_{\gamma=0}\left(\sum(-1)^{j}\left(b_{1, j}\left(M_{\nu_{0}}, Z\right)-\operatorname{dim} W_{a}^{j}\right)\right),
$$

where $b_{1, j}\left(M_{\nu_{0}}, Z\right)$ is a constant coefficient (i.e., the $t^{0}$-coefficient) of the asymptotic expansion of $\operatorname{Tr}\left(p_{1} \exp \left(-t \Delta_{\nu_{0}, j}\right)\right)$ relative to $t \rightarrow+0$ and $p_{1} \exp \left(-t \Delta_{\nu_{0}, j}\right)$ is the operator acting in $\left(D R^{j}(M)\right)_{2}$.

Remark 2.2. Note that in the right side of (2.16) there are the Euler characteristic $\chi\left(M_{\nu}, Z\right):=\sum(-1)^{j} \operatorname{dim} W_{a}^{j}$ and the alternating sum of the integrals $b_{1, j}\left(M_{\nu_{0}}, Z\right)$ (over $M_{1}$ and over $\partial M_{1}$ ) of the locally defined densities (Remark 2.1). (Here $Z$ is the union of the connected components of $\partial M$, where the Dirichlet boundary conditions are given). The number $\chi\left(M_{\nu}, Z\right)$ is also equal to the sum of the integrals over $M$, $N$, and over $\partial M$ of the locally defined densities.

Let $\nu(\gamma)$ be a smooth variation of a point $\nu_{0} \in U(2.3)$. Let $l(\gamma) \in \operatorname{det} W_{a}^{\bullet}(\nu(\gamma))$ be a variation of an arbitrary nonzero element $l \in \operatorname{det} W_{a}^{\bullet}\left(\nu_{0}\right)$ such that $\varphi_{\nu(\gamma)}^{a n}(a) \circ l(\gamma)$ is a fixed (nonzero) element of $\operatorname{Det}(M, N, Z)$. Then the equality (2.10) (where the factor $c_{0}(\nu)$ is constant on each connected component of $\left.U(2.3)\right)$ is equivalent to the 
assertion that for any such a variation $l(\gamma)$ its analytic torsion norm is independent of $\gamma$ :

$$
\left.\partial_{\gamma}\|l(\gamma)\|_{T_{0}\left(M_{\nu(\gamma)}, Z ; a\right)}^{2}\right|_{\gamma=0}=0
$$

Corollary 2.1 provides us with the formula (2.16) for a variation of the analytic torsion norm $\left\|g_{\nu(\gamma)} l\right\|_{T_{0}\left(M_{\nu(\gamma)}, Z ; a\right)}^{2}$ (where $l \in \operatorname{det} W_{a}^{\bullet}\left(\nu_{0}\right)$ ). The assertion (2.17) is equivalent to the following identity:

$$
\partial_{\gamma} \log \left\|\left.\left.g_{\nu(\gamma)} l\right|_{T_{0}\left(M_{\nu(\gamma)}, Z ; a\right)} ^{2}\right|_{\gamma=0}=\partial_{\gamma} \log \right\| g_{\nu *} f \|\left.^{2}\right|_{\gamma=0}
$$

where $f$ is an arbitrary nonzero element of $\operatorname{Det}(M, N, Z)$ (for instance, $f=\varphi_{\nu_{0}}^{a n}(a) \circ l$ ) and $g_{\nu *}=\varphi_{\nu}^{a n}(a) \circ g_{\nu} \circ\left(\varphi_{\nu_{0}}^{a n}(a)\right)^{-1}$ is defined by the following commutative diagram, where $\nu \in U$ is very close to $\nu_{0}$ :

$$
\begin{array}{ccc}
\operatorname{Det}(M, N, Z) & \underset{g_{\nu *}}{\longrightarrow} & \operatorname{Det}(M, N, Z) \\
\varphi_{\nu_{0}}^{a n}(a) \uparrow \imath \iota & \imath \imath \uparrow \varphi_{\nu}^{a n}(a) \\
\operatorname{det} W_{a}^{\bullet}\left(\nu_{0}\right) & \underset{g_{\nu}=g_{\nu \nu_{0}}}{\longrightarrow} & \operatorname{det} W_{a}^{\bullet}(\nu)
\end{array}
$$

The norm on the right in the equality (2.18) is an arbitrary Hilbert norm in onedimensional space $\operatorname{Det}(M, N, Z)$. The value of the expression on the right in $(2.18)$ is independent of such a norm.

The action of the isomorphism $g_{\nu}=g_{\nu \nu_{0}}: W_{a}^{\bullet}\left(\nu_{0}\right) \rightarrow W_{a}^{\bullet}(\nu)$ on $\operatorname{Det}(M, N, Z)$ is described by the following lemma.

Lemma 2.3. For an arbitrary element $f \in \operatorname{Det}(M, N, Z)$ the equality holds:

$$
\left.\partial_{\gamma} \log \left\|g_{\nu *} f\right\|^{2}\right|_{\gamma=0}=-\left.2 \partial_{\gamma}\left(\log k_{\nu}\right)\right|_{\gamma=0} \sum(-1)^{j} b_{2, j}\left(M_{\nu_{0}}, Z\right)
$$

where $b_{2, j}\left(M_{\nu_{0}}, Z\right)$ is the constant coefficient (i.e., the $t^{0}$-coefficient) in the asymptotic expansion (relative to $t \rightarrow+0$ ) for the trace of the operator $p_{2} \exp \left(-t \Delta_{\nu_{0}, j}\right)$ acting in $\left(D R^{j}(M)\right)_{2}$.

Here $p_{2}$ is the operator $p_{2}:\left(\omega_{1}, \omega_{2}\right) \rightarrow\left(0, \omega_{2}\right)$ for $\omega_{k} \in\left(D R\left(\bar{M}_{k}\right)\right)_{2}$.

Remark 2.3. Note that $\operatorname{Tr} \exp \left(-t \Delta_{\nu_{0}, j}\right)=\sum_{j} \operatorname{Tr}\left(p_{j} \exp \left(-t \Delta_{\nu_{0}, j}\right)\right)$. So we have

$$
-\sum(-1)^{j} b_{2, j}\left(M_{\nu_{0}}, Z\right)=\sum(-1)^{j} b_{1, j}\left(M_{\nu_{0}}, Z\right)-\chi\left(M_{\nu_{0}}, Z\right) .
$$

Hence the equality (2.18) follows from (2.16) and (2.19).

Thus Lemmas 2.1-2.3 provide us with a proof of the assertion that the factor $c_{0}(\nu)$ is independent of $\nu$ on each connected component $U_{j}$ of $U(2.3)$. 
2.2. Continuity of the analytic torsion norms. To prove that $c_{0}(\nu)$ is independent of $\nu \in \mathbb{R}^{2} \backslash(0,0)$, it is enough ${ }^{15}$ to show that the norm $\varphi_{\nu}^{a n} \circ T_{0}\left(M_{\nu}\right)$ on $\operatorname{Det}(M, N, Z)$ is continuous in $\nu \in \mathbb{R}^{2} \backslash(0,0)$. The following norms on $\operatorname{Det}(M, N, Z)$ are the same for an arbitrary $a \geq 0$ :

$$
\varphi_{\nu}^{a n}(a) \circ T_{0}\left(M_{\nu}, Z ; a\right)=\varphi_{\nu}^{a n} \circ T_{0}\left(M_{\nu}, Z\right) .
$$

Let us prove the continuity of $\varphi_{\nu}^{a n} T_{0}\left(M_{\nu}, Z\right)$ as a function of $\nu$ at a point $\nu_{0} \in$ $\mathbb{R}^{2} \backslash(0,0)$. (The series of lemmas above provides us with the proof of this assertion in the case when $\nu_{0} \in U(2.3)$. But now this will be proved at an arbitrary $\nu_{0} \in \mathbb{R}^{2} \backslash(0,0)$, for instance, at $\nu_{0} \in \mathbb{R}^{2} \backslash(U \cup(0,0))$.) By $(2.20)$, it is enough to obtain the continuity in $\nu$ at $\nu=\nu_{0}$ of the norm $\varphi_{\nu}^{a n}(a) \circ T_{0}\left(M_{\nu} ; a\right)$ on $\operatorname{Det}(M, N, Z)$ for a fixed $a>0$ such that $a \notin S\left(\nu_{0}\right):=\cup_{j} \operatorname{Spec}\left(\Delta_{\nu_{0}, j}\right)$. Since $a \notin S\left(\nu_{0}\right)$, we see that $a \notin S(\nu)$ for $\nu$ very close to $\nu_{0}$. (The latter assertion follows from Proposition 3.1. It claims that the resolvents $G_{\lambda}^{\bullet}(\nu):=\left(\Delta_{\nu}^{\bullet}-\lambda\right)^{-1}$ for $\lambda \notin \operatorname{Spec}\left(\Delta_{\nu, \bullet}\right)$ form a smooth in $(\lambda, \nu)$ family of bounded operators in $\left(D R^{\bullet}(M)\right)_{2}$ and that $\operatorname{Spec}\left(\Delta_{\nu, \bullet}\right)$ is discrete. As $G_{a}^{\bullet}\left(\nu_{0}\right)$ is bounded in $\left(D R^{\bullet}(M)\right)_{2}$, the operator $G_{a}^{\bullet}(\nu)$ is also bounded for $\nu$, close to $\nu_{0}$, and so $a \notin \operatorname{Spec}\left(\Delta_{\nu, \bullet}\right)$ for such $\nu$.) The assertion below claims that the truncated scalar analytic torsion (2.11) is a locally continuous function. ${ }^{16}$

Proposition 2.1. The scalar analytic torsion $T\left(M_{\nu}, Z ; a\right)$ is continuous in $\nu$ at $\nu_{0}$.

Thus, the continuity of $\varphi_{\nu}^{a n} T_{0}\left(M_{\nu}, Z\right)$ (as a function of $\nu$ ) at $\nu_{0}$ is equivalent to the condition that the norm on $\operatorname{Det}(M, N, Z)$

$$
\varphi_{\nu}^{a n}(a) \circ\|\cdot\|_{\operatorname{det} W_{a}^{\bullet}(\nu)}^{2}
$$

is continuous in $\nu$ at $\nu_{0}$. The continuity of the norm (2.21) is deduced from the following finite-dimensional algebraic lemma. Let

$$
f:\left(A^{\bullet}, d_{A}\right) \rightarrow\left(V^{\bullet}, d_{V}\right)
$$

be a quasi-isomorphism of finite complexes of finite-dimensional Hilbert spaces. Let $f_{*}$ : $\operatorname{det} H^{\bullet}(A) \underset{\sim}{\sim} \operatorname{det} H^{\bullet}(W)$ be the induced identification of the determinant lines. Let $T_{0}\left(A^{\bullet}\right)$ and $T_{0}\left(V^{\bullet}\right)$ be the analytic torsion norms (1.2) on the determinant lines identified by $f_{*}$ : $\operatorname{det} H^{\bullet}(A)=\operatorname{det} H^{\bullet}(V)$. Let $\left(\right.$ Cone $\left.^{\bullet} f, d\right)$, Cone $^{j} f=A^{j-1} \oplus V^{j}$, be a simple complex, associated with the bicomplex (2.22):

$$
d_{\text {Cone }}: \text { Cone }^{j} \rightarrow \text { Cone }^{j+1}, \quad d_{\text {Cone }}(x, y)=\left(-d_{A} x, f x+d_{V} y\right)
$$

\footnotetext{
${ }^{15}$ The factor $c_{0}(\nu)$ is constant on each connected component $U_{j}$ of $U(2.3)$, and $U$ is dense in $\mathbb{R}^{2} \backslash(0,0)$.

${ }^{16}$ This truncated scalar analytic torsion is a continuous function on the set of $\nu \in \mathbb{R}^{2} \backslash(0,0)$ such that $a \notin \cup_{i} \operatorname{Spec}\left(\Delta_{\nu, i}\right)$.
} 
(for $(x, y) \in A^{j+1} \oplus V^{j}$ ). Then Cone $f$ is an acyclic finite complex of finite-dimensional Hilbert spaces (Cone ${ }^{j} f$ is the direct sum of Hilbert spaces $A^{j+1}$ and $\left.V^{j}\right), H^{\bullet}($ Cone $f)=$ 0 . Hence $\operatorname{det} H^{\bullet}$ (Cone $f$ ) is canonically identified with $\mathbb{C}$ and the analytic torsion norm for Cone $f$ is a norm on $\mathbb{C}$. The ratio $T_{0}(V) / T_{0}(A) \in \mathbb{R}_{+}$is defined as the ratio between the two norms on the one-dimensional spaces $\operatorname{det} H^{\bullet}(V)$ and $\operatorname{det} H^{\bullet}(A)$ identified by $f_{*}$.

Lemma 2.4. Under the conditions above, the equality holds:

$$
\left.\|1\|_{T_{0}(\text { Cone }}^{2} f\right)=T_{0}\left(V^{\bullet}\right) / T_{0}\left(A^{\bullet}\right)
$$

where the left side is the analytic torsion norm of $1 \in \mathbb{C}=\operatorname{det} H^{\bullet}($ Cone $f)$.

Let $a>0$ be a number from $\mathbb{R}_{+} \backslash S\left(\nu_{0}\right)$. Then there exists an open neighborhood $U_{\nu_{0}}(a)$ of $\nu_{0} \in U_{\nu_{0}}(a) \subset \mathbb{R}^{2} \backslash(0,0)$ such that $a \notin S(\nu)$ for $\nu \in U_{\nu_{0}}(a)$ (Proposition 3.1). The family of complexes $\left(W_{a}^{\bullet}(\nu), d\right)$ of Hilbert spaces is continuous on $U_{\nu_{0}}(a)$ in the following sense.

The operator $\Pi_{a}^{j}(\nu):=\Pi^{j}(\nu ; a)$ is a finite rank projection operator in $\left(D R^{j}(M)\right)_{2}$ with its image $W_{a}^{j}(\nu)$ :

$$
\Pi_{a}^{j}(\nu):\left(D R^{j}(M)\right)_{2} \rightarrow W_{a}^{j}(\nu) \subset D R^{j}\left(M_{\nu}, Z\right) \subset\left(D R^{j}(M)\right)_{2} .
$$

Proposition 2.2. The family of operators $\Pi_{a}^{\bullet}(\nu)$ is continuous in $\nu$ for $\nu \in U_{\nu_{0}}(a)$ with respect to the operator norm in $\left(D R^{\bullet}(M)\right)_{2}$. The same is true for the families

$$
\begin{aligned}
& d \Pi_{a}^{\bullet}(\nu):\left(D R^{\bullet}(M)\right)_{2} \rightarrow D R^{\bullet+1}\left(M_{\nu}\right) \subset\left(D R^{\bullet+1}(M)\right)_{2}, \\
& \delta \Pi_{a}^{\bullet}(\nu):\left(D R^{\bullet}(M)\right)_{2} \rightarrow D R^{\bullet-1}\left(M_{\nu}\right) \subset\left(D R^{\bullet-1}(M)\right)_{2} .
\end{aligned}
$$

These are the families of finite rank operators.

Proof. It follows from Proposition 3.1 that if $a \notin S\left(\nu_{0}\right)$ then there exists an $\varepsilon>0$ such that $(a-\varepsilon, a+\varepsilon) \cap S(\nu)=\emptyset$ for $\nu$ sufficiently close to $\nu_{0}$. Hence $\{\lambda: a-\varepsilon<$ $|\lambda|<a+\varepsilon\} \cap S(\nu)=\emptyset$ for such $\nu$ (since $S(\nu) \subset \mathbb{R}_{+} \cup 0$ by Theorem 3.1). Thus, according to Proposition 3.1, the operators

$$
\Pi_{a}^{\bullet}(\nu)=\frac{i}{2 \pi} \int_{\Gamma_{a}} G_{\lambda}^{\bullet}(\nu) d \lambda
$$

form a smooth in $\nu$ (for such $\nu$ ) family of finite rank operators in $\left(D R^{\bullet}(M)\right)_{2}$ (where the circle $\Gamma=\{\lambda:|\lambda|=a\}$ is oriented opposite to the clockwise). The operators $d \Pi_{a}^{\bullet}(\nu):\left(D R^{\bullet}(M)\right)_{2} \rightarrow\left(D R^{\bullet+1}(M)\right)_{2}$ form (for such $\nu$ ) a smooth in $\nu$ family of finite rank operators (according to Proposition 3.1. 
Corollary 2.2. For $\nu$ sufficiently close to $\nu_{0}$ the family of operators $\Pi_{a}^{\bullet}(\nu)$ identifies the graded linear spaces $W_{a}^{\bullet}\left(\nu_{0}\right):=\operatorname{Im} \Pi_{a}^{\bullet}\left(\nu_{0}\right)$ and $W_{a}^{\bullet}(\nu)$. Such an identification nearly commutes with $d$ in the following sense:

$$
\left\|d \Pi_{a}^{\bullet}(\nu) w-\Pi_{a}^{\bullet+1}(\nu) d w\right\|_{2} \leq c\left(\nu, \nu_{0}\right)\|w\|_{2}
$$

(for any $w \in W_{a}^{\bullet}\left(\nu_{0}\right)$ ), where $c\left(\nu, \nu_{0}\right) \rightarrow+0$ as $\nu \rightarrow \nu_{0}$. This identification also nearly commutes with $\delta$ :

$$
\left\|\delta \Pi_{a}^{\bullet}(\nu) w-\Pi_{a}^{\bullet-1}(\nu) \delta w\right\|_{2} \leq c\left(\nu, \nu_{0}\right)\|w\|_{2}
$$

for $w \in W_{a}^{\bullet}\left(\nu_{0}\right)\left(\|\cdot\|_{2}\right.$ is the $L_{2}$-norm in $\left.\left(D R^{\bullet}(M)\right)_{2}\right)$.

The estimate (2.25) follows from the continuity (in $\nu$ ) of the families $d \Pi_{a}^{\bullet}(\nu)$ and $\Pi_{a}^{\bullet+1}(\nu)$ since the following operator norms tend to zero as $\nu \rightarrow \nu_{0}$ :

$$
\left\|d \Pi_{a}^{\bullet}(\nu)-d \Pi_{a}^{\bullet}\left(\nu_{0}\right)\right\|_{2} \rightarrow+0, \quad\left\|\Pi_{a}^{\bullet+1}(\nu)-\Pi_{a}^{\bullet+1}\left(\nu_{0}\right)\right\|_{2} \rightarrow+0 .
$$

Indeed, for an arbitrary $w \in W_{a}^{\bullet}\left(\nu_{0}\right)$ we have $d \Pi_{a}^{\bullet} w=d w$. Hence the estimates

$$
\begin{aligned}
\left\|\Pi_{a}^{\bullet+1}(\nu) d w-d w\right\|_{2} \leq\left\|\Pi_{a}^{\bullet+1}(\nu)-\Pi_{a}^{\bullet+1}\left(\nu_{0}\right)\right\|_{2} & \cdot\|d w\|_{2} \leq \\
& \leq C \cdot\left\|\Pi_{a}^{\bullet+1}(\nu)-\Pi_{a}^{\bullet+1}\left(\nu_{0}\right)\right\|_{2} \cdot\|w\|_{2}
\end{aligned}
$$

are true because the differential $d: W_{a}^{\bullet}\left(\nu_{0}\right) \rightarrow W_{a}^{\bullet+1}\left(\nu_{0}\right)$ of a finite complex of finitedimensional spaces is bounded (with respect to the Hilbert norm induced from $\left.\left(D R^{\bullet}(M)\right)_{2}\right)$.

For each $\nu \in \mathbb{R}^{2} \backslash(0,0)$ the combinatorial cochain complex $\left(C^{\bullet}\left(X_{\nu}, V\right), d\right)$ (with $V:=X \cap Z)$ is defined by the $\nu$-transmission condition (1.58). A homomorphism of the integration of forms from $W_{a}^{\bullet}(\nu)$ over the simplexes of $X$

$$
R_{\nu}(a):\left(W_{a}^{\bullet}(\nu), d\right) \rightarrow\left(C^{\bullet}\left(X_{\nu}, V\right), d\right)
$$

is also defined for all $\nu \in \mathbb{R}^{2} \backslash(0,0)$. For every such $\nu$ the following variant of the de Rham theorem holds.

Proposition 2.3. $R_{\nu}(a)$ is a quasi-isomorphism.

Proof. 1. Let $R_{\nu}:\left(D R^{\bullet}\left(M_{\nu}, Z\right), d\right) \rightarrow\left(C^{\bullet}\left(X_{\nu}, V\right), d\right)$ be the integration homomorphism of pairs of forms $\left(\omega_{1}, \omega_{2}\right) \in D R^{\bullet}\left(M_{\nu}, Z\right)$ over the simplexes of $X_{j} \backslash V_{j}$. Then $R_{\nu}$ is a quasi-isomorphism. ${ }^{17}$

\footnotetext{
${ }^{17}$ This assertion claims that the analogy of the classical de Rham theorem is true in the case of the $\nu$-transmission interior boundary conditions. The classical de Rham theorem for smooth closed manifolds was proved in [dR1] (see also [dR4], Ch. IV, [W], Ch. IV, § 29). The explicit isomorphism between the Cech cohomology for a good cover of a smooth closed $M$ and the de Rham cohomology of $M$ is defined with the help of the de Rham-Čech complex ([BT], Ch. II, § 9).
} 
Indeed, in the commutative diagram (1.59) the left and the right vertical arrows are quasi-isomorphisms according to the de Rham theorem for a closed manifold $N$ and for manifolds $M_{1}$ and $M_{2}$ with smooth boundaries. (The proof of the latter one is given in [RS], Proposition 4.2.) The cohomology exact sequences provide us with the commutative diagram

$$
\begin{array}{rrrrr}
\stackrel{\partial_{D}}{\longrightarrow} H^{*}\left(\oplus_{k=1,2} D R^{\bullet}\left(M_{k}, N \cup Z_{k}\right)\right) & \rightarrow & H^{*}\left(D R^{\bullet}\left(M_{\nu}, Z\right)\right) & \underset{\left(r_{\nu}\right)_{*}}{\longrightarrow} & H^{*}\left(D R^{\bullet}(N)\right) \rightarrow \\
\downarrow R_{*} & & \downarrow\left(R_{\nu}\right)_{*} & & \downarrow R_{*} \\
\stackrel{\partial_{c}}{\longrightarrow} H^{*}\left(\oplus_{k=1,2} C^{\bullet}\left(X_{k}, W \cup V_{k}\right)\right) & \underset{j_{*}}{\longrightarrow} & H^{*}\left(C^{\bullet}\left(X_{\nu}, V\right)\right) & \stackrel{\left(r_{\nu, c}\right)_{*}}{\longrightarrow} & H^{*}\left(C^{\bullet}(W)\right) \rightarrow
\end{array}
$$

with the exact rows, where the vertical arrows $R_{*}$ on the left and on the right are isomorphisms (according to the de Rham theorem) and where $\partial_{D}=\partial_{c}$ under the identifications $R_{*}$. Hence $\left(R_{\nu}\right)_{*}$ is also an isomorphism.

The exactness of the top row in (2.27) can be interpreted and proved as follows. The sheaf $F_{\nu}^{\bullet}:=D R_{\nu}^{\bullet}\left(\nu=(\alpha, \beta) \in \mathbb{R}^{2} \backslash(0,0)\right)$ of germs $\left(\omega_{1}, \omega_{2}\right)$ of pairs of $C^{\infty}$-forms $\omega_{j}$ on $M_{j}$ such that ${ }^{18} \alpha i_{1}^{*} \omega_{1}=\beta i_{2}^{*} \omega_{2}$ (here $i_{j}^{*}$ are the geometrical restrictions from $M_{j}$ to $N \hookrightarrow \partial M_{j}$ ) is a $c$-soft sheaf. (The latter notion means that the restriction $\Gamma\left(M, F_{\nu}^{j}\right) \rightarrow \Gamma\left(K, i_{K}^{-1} F_{\nu}^{j}\right)$ is surjective for any compact $i_{K}: K \hookrightarrow M,[\mathrm{KS}]$, Definition 2.5.5.) The sheaf $F_{\nu}$ is $c$-soft since appropriate smooth partitions of unity exist on $M$. The sequence of complexes of global sections

$$
0 \rightarrow \Gamma_{c}\left(M \backslash N, F_{\nu}^{\bullet}\right) \rightarrow \Gamma\left(M, F_{\nu}^{\bullet}\right) \rightarrow \Gamma\left(M, i_{N, *} i_{N}^{-1} F_{\nu}^{\bullet}\right) \rightarrow 0
$$

(here $i_{N}: N \hookrightarrow M$ ) has the terms which possess the following properties:

1) $\Gamma\left(M, F_{\nu}^{\bullet}\right)=D R^{\bullet}\left(M_{\nu}, Z\right)$

2) $\Gamma_{c}\left(M \backslash N, F_{\nu}^{\bullet}\right)$ is a subcomplex of $\oplus_{k=1,2} D R^{\bullet}\left(M_{k}, N \cup Z_{k}\right)$ and its natural inclusion is a quasi-isomorphism. Indeed, if $\omega \in D R^{\bullet}\left(M_{k}, N \cup Z_{k}\right)$ is a closed form then $\omega=d v$ in a neighborhood of $N$ in $M_{k}$ (where $v$ is a smooth form with the zero geometrical restriction to $N)$. So $\omega-d(\varphi v)=0$ in some neighborhood of $N$ in $M_{k}$ ( $\varphi$ is an appropriate cutting function). We have $\Gamma_{c}\left(M \backslash N, F_{\nu}^{\bullet}\right)=\Gamma\left(M, j_{!} j^{-1} F_{\nu}^{\bullet}\right)$, where $j: M \backslash N \hookrightarrow M$ and $j_{\text {! }}$ is the direct image with proper supports, $\left.j^{-1} F_{\nu}^{\bullet} \simeq D R^{\bullet}\right|_{M \backslash N}$. The sheaf $j ! j^{-1} F_{\nu}^{\bullet}$ is $c$-soft according to [KS], Proposition 2.5.7.

3) $\Gamma\left(M, i_{N, *} i_{N}^{-1} F_{\nu}^{\bullet}\right)$ has a natural homomorphism $q_{\nu}:=r_{\nu} \circ\left(i_{1}^{*}, i_{2}^{*}\right)$ onto $D R^{\bullet}(N)$ (where $r_{\nu}$ is defined in (1.15)) and $q_{\nu}$ is a quasi-isomorphism. In fact, if the form $u=d t \wedge \omega_{N}(t)$ on $I \times N$ is closed then it is exact, because then $d_{N} \omega_{N}(t)=0$ and so $u=d \int_{0}^{t} \omega_{N}(\tau) d \tau$. (Here $t$ is the coordinate on $I$ and $t=0$ is the equation of

\footnotetext{
${ }^{18}$ It is supposed that $\omega_{j}$ has the zero geometrical restriction to $Z_{k}$ (at the points $x \in Z_{k} \subset \partial M_{k}$ ).
} 
$N=0 \times N \hookrightarrow I \times N, 0 \in I \backslash \partial I$.) Hence $q_{\nu}$ is a quasi-isomorphism. (This assertion follows also from the Poincaré lemma.) The sheaf $i_{N, *} i_{N}^{-1} F_{\nu}^{\bullet}$ is $c$-soft by [KS], Proposition 2.5.7.

For a compact manifold $M$ the category of $c$-soft sheaves on $M$ is injective with respect to the functor of global sections $\Gamma(M ; \cdot)([\mathrm{KS}]$, Proposition 2.5.10). The complex $F_{\nu}^{\bullet}$ is a $c$-coft resolvent of a constructible sheaf ([KS], Chapter VIII) $\mathbb{C}_{\nu}$ on $M$, which is isomorphic to $\mathbb{C}_{M \backslash N}$ on $M \backslash N$ and to $\mathbb{C}_{N}$ on $N$ (where $\mathbb{C}_{X}$ is a constant sheaf on $X$ ), and the gluing map for $\mathbb{C}_{\nu}$ is $|\nu|^{-1 / 2}(\alpha, \beta): \mathbb{C}_{N} \rightarrow i_{N}^{-1} j_{*} \mathbb{C}_{M \backslash N}=\mathbb{C}_{N} \oplus \mathbb{C}_{N}$ (i.e., $\left.c \rightarrow|\nu|^{-1}(\beta c, \alpha c)\right)$. The complexes $j_{!} j^{-1} F_{\nu}^{\bullet}$ and $i_{N, *} i_{N}^{-1} F_{\nu}^{\bullet}$ are $c$-soft resolvents of constructible sheaves $j_{!} j^{-1} \mathbb{C}_{\nu}=j_{!} \mathbb{C}_{M \backslash N}$ and of $i_{N, *} i_{N}^{-1} \mathbb{C}_{\nu}$. (The latter one is isomorphic to $i_{N, *} \mathbb{C}_{N}$ under $r_{\nu}$.) So the exactness of the cohomology sequence in the top row of (2.27) follows from [KS], (2.6.33), Remark 2.6.10.

2. The projection operator $p_{\mathcal{H}}: D R^{\bullet}\left(M_{\nu}, Z\right) \hookrightarrow\left(D R^{\bullet}(M)\right)_{2} \rightarrow \operatorname{Ker}\left(\Delta_{\nu}^{\bullet}\right)$ provides us with the isomorphism $p_{\mathcal{H} *}: H^{\bullet}\left(D R\left(M_{\nu}, Z\right)\right) \rightarrow \operatorname{Ker}\left(\Delta_{\nu}^{\bullet}\right)$ (by Lemma 1.1). So the inclusion $i_{a}:\left(W_{a}^{\bullet}(\nu), d\right) \hookrightarrow\left(D R^{\bullet}\left(M_{\nu}, Z\right), d\right)$ is a quasi-isomorphism and $\left(i_{a}\right)_{*}: \operatorname{Ker}\left(\Delta_{\nu}^{\bullet}\right) \underset{\sim}{\sim} H^{\bullet}\left(D R\left(M_{\nu}, Z\right)\right)$ is equal to $\left(p_{\mathcal{H}_{*}}\right)^{-1}$ (since $p_{\mathcal{H}} i_{a}=$ id on $\left.\operatorname{Ker}\left(\Delta_{\nu}^{\bullet}\right)\right)$. From an obvious equality $R_{\nu}(a)=R_{\nu} i_{a}$ it follows that $R_{\nu}(a)$ is a quasi-isomorphism.

Thus the assertion of Lemma 2.4 can be applied to the bicomplex (2.26). The result is as follows.

Corollary 2.3. The equality holds:

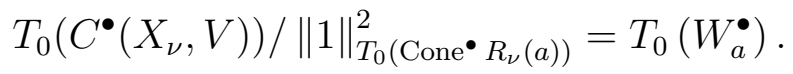

The identifications $\varphi_{\nu}^{a n}(a)$ (for an arbitrary $a>0$ ) and $\varphi_{\nu}^{a n}$ are defined such that the following norms on $\operatorname{Det}(M, N, Z)$ are equal:

$$
\varphi_{\nu}^{a n}(a) \circ\|\cdot\|_{\operatorname{det} W_{a}^{*}(\nu)}^{2}=\varphi_{\nu}^{a n} \circ T_{0}\left(W_{a}\right) .
$$

Hence, as it follows from (2.28), (2.29), we have

$$
\varphi_{\nu}^{a n}(a) \circ\|\cdot\|_{\operatorname{det} W_{a}^{*}(\nu)}^{2}=\left(\varphi_{\nu}^{a n} \circ T_{0}\left(C^{\bullet}\left(X_{\nu}, V\right)\right)\right) \circ\left(\|1\|^{2}\right)_{T_{0}\left(\operatorname{Cone}_{\bullet}\left(R_{\nu}(a)\right)\right)}^{-1}
$$

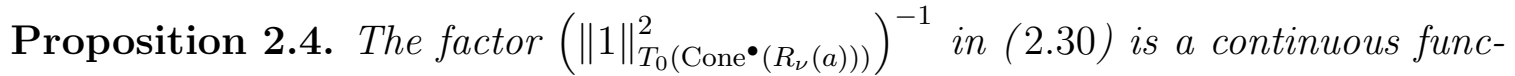
tion of $\nu \in U_{\nu_{0}}(a)$.

Proof. The complex Cone ${ }^{\bullet}\left(R_{\nu}(a)\right)$ is acyclic according to Proposition 2.3. Its scalar analytic torsion

$$
\|1\|_{T_{0}\left(\mathrm{Cone} \cdot\left(R_{\nu}(a)\right)\right)}^{2}:=\exp \left(\sum_{j \geq-1}(-1)^{j} j \zeta_{j}^{\prime}(0)\right)
$$


is defined as in (1.1) by the $\zeta$-functions of the "Laplacians" $L_{\nu}:=d_{\nu}^{*} d_{\nu}+d_{\nu} d_{\nu}^{*}$ of the complex (Cone $\left.{ }^{\bullet}\left(R_{\nu}(a)\right), d_{\nu}\right){ }^{19}$ Since the complex Cone ${ }^{\bullet}\left(R_{\nu}(a)\right)$ is acyclic we see that these Laplacians are positive definite. (So they have the zero kernels.) Their determinants $\operatorname{det}\left(\Delta_{\nu}^{\bullet}\right)$ are continuous positive functions of $\nu$ on $U_{\nu_{0}}(a)$ (and so the expression on the right in (2.31) is a continuous function of $\left.\nu \in U_{\nu_{0}}(a)\right)$. The latter statement is derived as follows.

Proposition 2.5. Let $m \in \mathbb{Z}_{+}$and $m \geq m_{0}:=1+\min \left\{k \in \mathbb{Z}_{+}: 4 k \geq \operatorname{dim} M\right\}$. Then there exists a positive constant $C=C\left(M, N, Z, g_{M}\right)$ independent of $\nu \in \mathbb{R}^{2} \backslash$ $(0,0)$ (and of $m$ also) such that the following estimate holds uniformly with respect to $x \in \bar{M}_{1} \cup \bar{M}_{2}$ :

$$
|\omega(x)|^{2}<C \sum_{i=0}^{m}\left\|\Delta_{\nu}^{i} \omega\right\|_{2}^{2}
$$

for all $\omega$ such that ${ }^{20}$

$$
\omega \in D R^{\bullet}\left(M_{\nu}, Z\right), \quad \omega \in D\left(\Delta_{\nu}^{\bullet}\right), \quad \Delta_{\nu} \omega \in D\left(\Delta_{\nu}^{\bullet}\right), \ldots, \Delta_{\nu}^{m} \omega \in D\left(\Delta_{\nu}^{\bullet}\right) .
$$

(Here $|\omega(x)|^{2}$ is the norm at $\wedge^{\bullet} T_{x} M$ defined by $g_{M}$ and $\|\cdot\|_{2}^{2}$ is the $L_{2}$-norm in $\left(D R^{\bullet}(M)\right)_{2}$.)

Corollary 2.4. If $w \in W_{a}^{\bullet}(\nu)$ then $w \in D\left(\Delta_{\nu}^{m}\right)$ for an arbitrary $m \in \mathbb{Z}_{+}$. So the following estimate holds uniformly with respect to $x \in \bar{M}_{1} \cup \bar{M}_{2}$ and to $\nu \in \mathbb{R}^{2} \backslash(0,0)$

$$
|w(x)|^{2}<C_{1}\|w\|_{2}^{2},
$$

where $C_{1}=C_{1}\left(M, N, Z, g_{M}\right)>0$.

The graded Hilbert space $C^{\bullet}\left(X_{\nu}, V\right)$ is isomorphic to the direct sum

$$
C^{\bullet}\left(X_{\nu}, V\right)=C^{\bullet}\left(X, N_{X} \cup V\right) \oplus C^{\bullet}\left(N_{X}\right),
$$

where $V:=X \cap Z, N_{X}:=X \cap N, C^{\bullet}\left(X, N_{X} \cap V\right)$ is a graded linear subspace of $C^{\bullet}\left(X_{\nu}, V\right)$ (with respect to the natural inclusion), and the inclusion $j_{\nu}: C^{\bullet}\left(N_{X}\right) \hookrightarrow$ $C^{\bullet}\left(X_{\nu}, V\right) \subset \oplus_{i} C^{\bullet}\left(X_{i}\right)$ is defined as $j_{\nu}:=\left(\alpha^{2}+\beta^{2}\right)^{-1}(\beta$ id, $\alpha$ id $)$. The space on the right in (2.35) is independent of $\nu$. Hence (2.35) provides us with the isometric identification of the graded Hilbert spaces

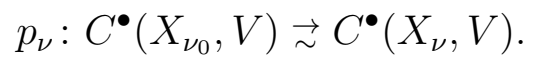

\footnotetext{
${ }^{19}$ The spaces $W_{a}^{\bullet}(\nu)$ are equiped with the Hilbert structure from $\left(\left(D R^{\bullet}(M)\right)_{2}, g_{M}\right)$. The spaces $C^{\bullet}\left(X_{\nu}, V\right) \subset C^{\bullet}\left(X_{1}\right) \oplus C^{\bullet}\left(X_{2}\right)$ are equiped with the Hilbert structure defined by the basic cochains in $\oplus C^{\bullet}\left(X_{k}\right)$ and $\operatorname{Cone}^{\bullet}\left(R_{\nu}\right)=W_{a}^{\bullet-1}(\nu) \oplus C^{\bullet}\left(X_{\nu}, V\right)$ is the orthogonal direct sum of Hilbert spaces.

${ }^{20}$ The domain of definition of $D\left(\Delta_{\nu}^{\bullet}\right)$ for $\Delta_{\nu}^{\bullet}$ is defined by (1.27) and (1.26).
} 
Corollary 2.5. Let $\nu \in U_{\nu_{0}}(a)$ be sufficiently close to $\nu_{0}$. Let $W_{a}^{\bullet}(\nu)$ be identified with $W_{a}^{\bullet}\left(\nu_{0}\right)$ by $\Pi_{a}^{\bullet}(\nu): W_{a}^{\bullet}\left(\nu_{0}\right) \rightarrow W_{a}^{\bullet}(\nu)$. Let $C^{\bullet}\left(X_{\nu_{0}}, V\right)$ be identified with $C^{\bullet}\left(X_{\nu}, V\right)$ by $p_{\nu}$ (2.36). Then the estimate (2.34) involves that for such $\nu$ the family of homomorphisms of the integration over the simplexes of $X$

$$
R_{\nu}^{\bullet}(a):\left(W_{a}^{\bullet}(\nu), d\right) \rightarrow\left(C^{\bullet}\left(X_{\nu}, V\right), d_{c}\right)
$$

is a continuous in $\nu$ family of quasi-isomorphisms between finite complexes of finitedimensional Hilbert spaces.

Let $f_{\nu}:\left(F^{\bullet}(\nu), d_{F}(\nu)\right) \rightarrow\left(K^{\bullet}(\nu), d_{K}(\nu)\right)$ be a family of homomorphisms between finite complexes of finite-dimensional Hilbert spaces. Let the trivialization of these two families of complexes be defined by the identifications of the graded linear spaces

$$
\Pi_{\nu}: F^{\bullet}\left(\nu_{0}\right) \rightarrow F^{\bullet}(\nu), \quad p_{\nu}: K^{\bullet}\left(\nu_{0}\right) \rightarrow K^{\bullet}(\nu) .
$$

Let these idetifications be chosen such that $f_{\nu}$ becomes a continuous family of the homomorphisms

$$
f_{\nu}:\left(F^{\bullet}, d_{F}(\nu)\right) \rightarrow\left(K^{\bullet}, d_{K}(\nu)\right)
$$

between the continuous families of complexes with the fixed underlying graded linear spaces $F^{\bullet}:=F^{\bullet}\left(\nu_{0}\right)$ and $K^{\bullet}:=K^{\bullet}\left(\nu_{0}\right)$. Let the Hilbert structures on $F^{j}$ and $K^{j}$ are continuous functions of $\nu$ for all $j$. In this case, $f_{\nu}$ is called a continuous family. Then the following assertion is true.

Proposition 2.6. Let $f_{\nu}$ be a continuous family. Then the determinants $\operatorname{det}\left(L_{\nu}^{\bullet}\right)$ of the Laplacians $L_{\nu}^{\bullet}=d_{\nu}^{*} d_{\nu}+d_{\nu} d_{\nu}^{*}$ on $\left(\mathrm{Cone}^{\bullet} f_{\nu}, d_{\nu}\right)$ are continuous functions of $\nu$.

Proof. The operator $d_{\nu}^{*}$ adjoint to the differential $d_{\nu}$ of Cone $f_{\nu}$ (relative to the Hilbert structure on Cone $\left.f_{\nu}=F^{\bullet-1} \oplus K^{\bullet}\right)^{21}$ is defined on the whole finite-dimensional space Cone $f_{\nu}$. Since $d_{\nu}$ tends to $d_{\nu_{0}}$ (for instance, in the operator norm ${ }^{22}$ ) as $\nu \rightarrow \nu_{0}$ we see that $d_{\nu}^{*}$ also tends to $d_{\nu_{0}}^{*}$. Thus $L_{\nu}^{\bullet} \rightarrow L_{\nu_{0}}^{\bullet}$ as $\nu \rightarrow \nu_{0}$ and $\operatorname{det} L_{\nu}^{\bullet} \rightarrow \operatorname{det} L_{\nu_{0}}^{\bullet}$ (since the space Cone $f_{\nu}$ is finite-dimensional).

Corollary 2.6. The functions $\operatorname{det}\left(L_{\nu}^{\bullet}\right)$ of $\nu$ for $f_{\nu}=R_{\nu}^{\bullet}(a)$ are continuous and positive.

The positivity of $\operatorname{det}\left(L_{\nu}^{\bullet}\right)$ is equivalent to the acyclicity of (Cone $\left.R_{\nu}(a), d_{\nu}\right)$ (where $\left.d_{\nu}:=d_{\operatorname{Cone}\left(R_{\nu}(a)\right)}\right)$.

Proposition 2.4 is proved.

\footnotetext{
${ }^{21}$ Cone ${ }^{\bullet} f$ is the direct sum of Hilbert spaces $F^{\bullet+1} \oplus K^{\bullet}$ (with the Hilbert structures on $F^{\bullet+1}$ and $K^{j}$ depending continuously on $\nu$ ).

${ }^{22}$ As Cone $f_{\nu}$ is a finite-dimensional space, the weak convergence of the operators acting in it is equivalent to the convergence with respect to the operator norm.
} 
Remark 2.4. Propositions 2.2, 2.5, and Corollary 2.5 claim that under the identifications (2.36) and $\Pi_{a}^{\bullet}(\nu)$, the Hilbert structures on det Cone ${ }^{\bullet}\left(R_{\nu}(a)\right)$ and the differentials $d_{\nu}$ in Cone ${ }^{\bullet}\left(R_{\nu}(a)\right)$ are continuous in $\nu$ at $\nu_{0}$. Hence the analytic torsion norms

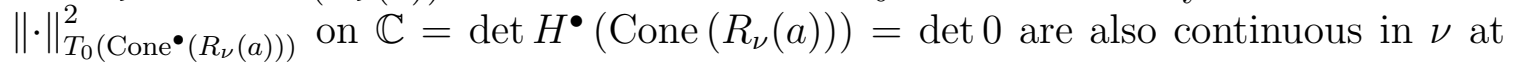
$\nu_{0}$.

According to (1.61) we have $\varphi_{\nu}^{a n}=\varphi_{\nu}^{c}$, where $\varphi_{\nu}^{c}$ is defined by the bottom row of the commutative diagram (1.59). So the continuity of the norm $\varphi_{\nu}^{a n}(a) \circ\|\cdot\|_{\operatorname{det} W_{a}^{\bullet}(\nu)}^{2}$ on $\operatorname{Det}(M, N, Z)$ can be deduced from (2.30) and from the following lemma.

Lemma 2.5. The norm $\varphi_{\nu}^{c} T_{0}\left(C\left(X_{\nu}, V\right)\right)$ on $\operatorname{Det}(M, N, Z)$ does not depend on $\nu \in$ $U_{\nu_{0}}(a)$.

The continuity in $\nu$ of the norm $\varphi_{\nu}^{a n} T_{0}\left(M_{\nu}, Z\right)$ on $\operatorname{Det}(M, N, Z)$ follows from $(2.20)$, $(2.21)$, and from the continuity of the norm $\varphi_{\nu}^{a n}(a) \circ\|\cdot\|_{\operatorname{det} W_{a}(\nu)}^{2}$. (The latter assertion is proved above.) The equality (1.12) holds with $c_{0}(\nu)$ which is constant and positive on each connected component $U_{j}$ of $U(2.3)$. Because the norm $\varphi_{\nu}^{a n} T_{0}\left(M_{\nu}\right)$ on $\operatorname{Det}(M, N, Z)$ is continuous in $\nu \in \mathbb{R}^{2} \backslash(0,0)$, the equality (1.12) holds for all such $\nu$ with $c_{0}$ independent of $\nu$. Theorem 1.1 follows from (1.12) and from the assertion of Lemma 1.2.

Remark 2.5. It is not important for the proofs of Theorem 1.1 and of (1.12) that the family of finite-dimensional complexes $\left(C^{\bullet}\left(X_{\nu}, V\right), d_{c}\right)$ in $(2.30)$ is of a combinatorial nature. It is enough for the proof to have a family of finite-dimensional complexes $\left(F_{\nu}^{\bullet}, d_{F}\right)$ which are defined locally in $\nu$ (i.e., for $\nu$ in a neighborhood of an arbitrary $\left.\nu_{0} \in \mathbb{R}^{2} \backslash(0,0)\right)$ together with the data as follows. Continuous families of quasiisomorphisms $f_{\nu}(a):\left(W_{a}^{\bullet}(\nu), d\right) \rightarrow\left(F_{\nu}^{\bullet}, d_{F}\right)$ and of Hilbert structures $h_{\nu}$ on $F_{\nu}^{\bullet}$ are defined. A family $\left(F_{\nu}^{\bullet}, h_{\nu}\right)$ may depend on $a$ and on $\nu_{0}$ but it has to possess the property as follows. The norm $\varphi_{\nu}^{a n} \circ\left(f_{\nu}(a)_{*}\right)^{-1} \circ T_{0}\left(F_{\nu}^{\bullet}, h_{\nu}\right)$ on $\operatorname{Det}(M, N, Z)$ is

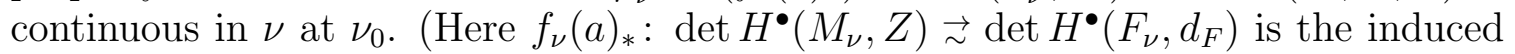
identification.)

Proof of Lemma 2.5. Let $\psi_{\nu}$ be the identification of the determinant lines defined by the bottom row of the commutative diagram (1.59):

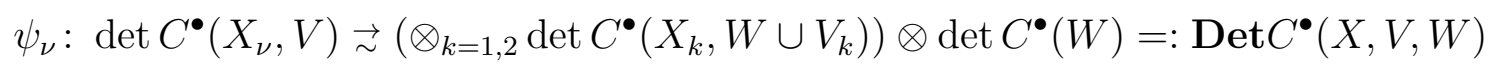

(where $V$ is the induced smooth triangulation $Z \cap X$ of $Z \subset \partial M, V_{k}:=V \cap \partial M_{k}$ 
and $W:=X \cap N=N_{X}$ ). The following diagram is commutative:

$$
\begin{array}{ccc}
\operatorname{det} C^{\bullet}\left(X_{\nu}, V\right) & \stackrel{\psi_{\nu}}{\longrightarrow} & \operatorname{Det} C^{\bullet}(X, V, W) \\
d_{c} \downarrow \imath & d_{c} \downarrow \\
\operatorname{det} H^{\bullet}\left(X_{\nu}, V\right) \stackrel{\varphi_{\nu}^{c}}{\longrightarrow} & \operatorname{Det}(X, V, W) \\
\|_{R} & \|_{R} \\
\operatorname{det} H^{\bullet}\left(M_{\nu}, Z\right) \stackrel{\varphi_{\nu}^{a n}}{\longrightarrow} & \operatorname{Det}(M, Z, N)
\end{array}
$$

(The determinant lines on the right in (2.37) are defined by (1.54). The identification $d_{c}$ on the right in (2.37) is a triple tensor product of the identifications induced by $d_{c}$ on $C^{\bullet}\left(X_{k}, W \cup V_{k}\right)$ and on $C^{\bullet}(W)$. The identification $R$ is defined by the integration over the simplexes of $X$.) The commutativity of the diagram (2.37) is equivalent to the definition (1.60) of $\varphi_{\nu}^{c}$. Since the identification $d_{c}$ on the right in (2.37) is independent of $\nu$ we see that the statement of Lemma 2.5 is a consequence of the following proposition.

Proposition 2.7. The identification $\psi_{\nu}$ in (2.37) is an isometry between the combinatorial norm $\|\cdot\|_{\operatorname{det} C \cdot\left(X_{\nu}, V\right)}^{2}$ and the triple tensor product of the combinatorial norms on $\operatorname{det} C^{\bullet}\left(X_{k}, W \cup V_{k}\right)(k=1,2)$ and on $\operatorname{det} C^{\bullet}(W)$.

(The Hilbert structures on $\oplus_{k=1,2} C^{\bullet}\left(X_{k}, V_{k}\right)$ and on $C^{\bullet}(W)$ are defined by the orthonormal basis of the basic cochains.)

Proof. Let $\rho_{\nu, c}: C^{\bullet}(W) \rightarrow C^{j}\left(X_{\nu}, V\right)$ be defined by (1.63). Then $r_{\nu, c} \rho_{\nu, c}=\mathrm{id}$ and $\rho_{\nu, c}$ is an isometry onto $\operatorname{Im}\left(\rho_{\nu, c}\right)$ (relative to the Hilbert structures, defined above). The subspace $\operatorname{Im}\left(\rho_{\nu, c}\right)$ is the orthogonal complement to $\operatorname{Im} j\left(\oplus_{k=1,2} C^{\bullet}\left(X_{k}, W \cup V_{k}\right)\right)$ in $C^{j}\left(X_{\nu}, V\right)$ and $j$ is an isometry onto $\operatorname{Im} j$. (Here, $r_{\nu, c}$ and $j$ are the same as in the bottom row of (1.59))..$^{23}$.

Thus Lemma 2.5 is proved.

2.2.1. Uniform Sobolev inequalities for $\nu$-transmission interior boundary conditions. Proof of Proposition 2.5. Let $I \times N \subset M$ (where $I=[-1,1]$ ) be a neighborhood of $N=0 \times N \subset M$ and let $g_{M}$ be a direct product metric on $I \times N$. Proposition 2.5 is a consequence of the assertions as follows.

Proposition 2.8. The inequality (2.32) holds uniformly with respect to $\nu \in \mathbb{R}^{2} \backslash(0,0)$ for all $\omega \in D R^{\bullet}\left(M_{\nu}\right)$ of the class (2.33) and such that supp $\omega \subset[-4 / 5,4 / 5] \times N \subset M$.

\footnotetext{
${ }^{23}$ This proposition is essentially equivalent to Proposition 1.5 , Section 1.
} 
Proposition 2.9. The inequality (2.32) holds for all $\omega \in D R^{\bullet}(M, Z)$ such that supp $\omega \subset M \backslash([-1 / 3,1 / 3] \times N)$ and such that ${ }^{24}$

$$
\omega \in D\left(\Delta_{M, Z}\right), \Delta \omega \in D\left(\Delta_{M, Z}\right), \ldots, \Delta^{m} \omega \in D\left(\Delta_{M, Z}\right) .
$$

The last assertion is well known ([Ch], Section 5).

Let $f$ be a smooth function on $M, 0 \leq f \leq 1, f \equiv 1$ on $[-1 / 2,1 / 2] \times N$ and $f \equiv 0$ on $M \backslash([-3 / 4,3 / 4] \times N)$. The $2 m$-Sobolev norm on the right in $(2.32)$, defined as

$$
\|\omega\|_{(2 m)}^{2}:=\sum_{k=0}^{m}\left\|\Delta_{\nu}^{k} \omega\right\|_{2}^{2},
$$

is equivalent uniformly in $\nu \in \mathbb{R}^{2} \backslash(0,0)$ (i.e., with constants $c_{3}, c_{4}>0$ independent of $\nu$ and of $\omega)$ to the norm ${ }^{25}:\|\omega\|_{(2 m), f}^{2}:=\sum_{k=0}^{m}\left(\left\|\Delta_{\nu}^{k}(f \omega)\right\|_{2}^{2}+\left\|\Delta^{k}((1-f) \omega)\right\|_{2}^{2}\right)$ :

$$
c_{3}\|\omega\|_{(2 m)}^{2}<\|\omega\|_{(2 m), f}^{2} \leq c_{4}\|\omega\|_{(2 m)}^{2} .
$$

It is enough to verify the upper estimate (with $c_{4}$ independent of $\nu$ ) for $f \omega$. It is true for $m=1$, since the estimate holds:

$$
\left\|\Delta_{\nu}(f \omega)\right\|_{2}^{2} \leq C_{1} \cdot\left(\left\|\Delta_{\nu} \omega\right\|_{2}^{2}+\|\omega\|_{2}^{2}+\|d \omega\|_{2}^{2}+\|\delta \omega\|_{2}^{2}\right) \leq 3 / 2 C_{1}\left(\left\|\Delta_{\nu} \omega\right\|_{2}^{2}+\|\omega\|_{2}^{2}\right)
$$

where $C_{1}$ depends on $f$ but it is independent of $\nu$. Hence the following estimate holds for $\omega \in D\left(\Delta_{\nu}^{k}\right)$ (with $C_{2}$ independent of $\nu$ and of $\omega$ ):

$$
\left\|\Delta_{\nu}^{k}(f \omega)\right\|_{2}^{2} \leq C_{2} \cdot\left(\left\|\Delta_{\nu}^{k} \omega\right\|_{2}^{2}+\left\|\Delta_{\nu}^{k-1} \omega\right\|_{2}^{2}+\ldots+\|\omega\|_{2}^{2}\right)
$$

The upper estimate is done. Thus Proposition 2.5 follows from (2.39) and from Propositions 2.8 and 2.9.

Proof of Proposition 2.8. The form $\omega$ on $I \times N$ is the sum $\omega_{0}+\omega_{1}$, where $\omega_{i}$ is an $i$-form in the direction of $I$ (where $I=[-1,1]$ ). It is enough to prove the inequality (2.32) separately for $\omega_{0}$ and for $\omega_{1}$. Let us prove it for $\omega_{0}$. For $\nu=(\alpha, \beta) \in \mathbb{R}^{2} \backslash(0,0)$ the Green function $G(\nu)$ for the Laplacian $\Delta_{\nu, I}$ on functions

\footnotetext{
${ }^{24}$ For $\omega$ with supp $\omega \subset M \backslash N$ the conditions (2.33) and (2.38) are equivalent. The domain $D\left(\Delta_{M, Z}\right)$ of $\Delta_{M, Z}$ consists of smooth forms on $M$ with the Dirichlet boundary conditions on $Z$ and the Neumann ones on $\partial M \backslash Z$.

${ }^{25}$ The lower estimate with $c_{3}$ in $(2.39)$ is obvious. Note that $\operatorname{supp}((1-f) \omega) \subset M \backslash$ $([-1 / 3,1 / 3] \times N)$. Then the upper estimate with a constant $c_{4}^{\prime}$ for $\|(1-f) \omega\|_{(2 m)}$ by $\|\omega\|_{(2 m)}$ is well known ([Hö], Appendix B and Proposition 20.1.11).
} 
on $I$ with the $\nu$-transmission boundary condition at $0 \in I$ and the Dirichlet boundary conditions on $\partial I=\{-1,1\}$ is given by the kernel

$$
\begin{aligned}
& \left(G_{I}(\nu)\right)_{x_{1}, x_{2}}=g_{x_{1}, x_{2}}+\frac{\beta^{2}-\alpha^{2}}{\alpha^{2}+\beta^{2}} g_{-x_{1}, x_{2}} \text { for } x_{1}, x_{2} \in Q_{1}=[-1,0], \\
& \left(G_{I}(\nu)\right)_{x_{1}, x_{2}}=g_{x_{1}, x_{2}}+\frac{\alpha^{2}-\beta^{2}}{\alpha^{2}+\beta^{2}} g_{-x_{1}, x_{2}} \text { for } x_{1}, x_{2} \in Q_{2}=[0,1], \\
& \left(G_{I}(\nu)\right)_{x_{1}, x_{2}}=\frac{2 \alpha \beta}{\alpha^{2}+\beta^{2}} g_{x_{1}, x_{2}} \quad \text { for } x_{1}, x_{2} \text { from different } Q_{k} .
\end{aligned}
$$

Here, $g_{x_{1}, x_{2}}$ is the Green function for the Laplacian on functions on $I$ with the Dirichlet boundary conditions on $\partial I$ :

$$
g_{x_{1}, x_{2}}= \begin{cases}c \cdot\left(x_{2}+1\right)\left(1-x_{1}\right), & -1 \leq x_{2} \leq x_{1} \leq 1 \\ c \cdot\left(x_{1}+1\right)\left(1-x_{2}\right), & -1 \leq x_{1} \leq x_{2} \leq 1\end{cases}
$$

where $c \neq 0$ is a constant.

It follows from $(2.40)$ and $(2.41)$ that $G_{I}(\nu)$ has a continuous kernel on $\bar{Q}_{r_{1}} \times \bar{Q}_{r_{2}}$ and that it is estimated uniformly with respect to $\nu \in \mathbb{R}^{2} \backslash(0,0)$ and to $x_{1}, x_{2}$ :

$$
\sup _{x_{1}, x_{2}, \nu}\left|\left(G_{I}(\nu)\right)_{x_{1}, x_{2}}\right|<c_{2}
$$

Since supp $\omega_{0} \subset(I \backslash \partial I) \times N$ and since the Laplacian $\Delta_{\nu, I}$ has the zero kernel on functions with the Dirichlet boundary conditions on $\partial I$, we have

$$
\omega_{0}=\left(\operatorname{id}_{I} \otimes \Pi_{0}^{\bullet}(N)\right) \omega_{0}+G_{I}(\nu) \otimes G_{N}^{m_{2}}\left(\left(\Delta_{\nu, I} \otimes \Delta_{N}^{m_{2}}\right) \omega_{0}\right),
$$

where $G_{N}$ is the Green function for $\Delta_{N}^{\bullet}$ and where $\Pi_{0}^{\bullet}(N)$ is the orthogonal projection operator in $\left(D R^{\bullet}(N)\right)_{2}$ onto Ker $\Delta_{N}^{\bullet}$. The operator $G_{N}^{m_{2}}$ on a closed Riemannian manifold $\left(N, g_{N}\right)$ has a square-integrable kernel (relative to the second argument) for $m_{2}>(n-1) / 4($ where $n-1=\operatorname{dim} N)$ and it has a continuous on $N \times N$ kernel for $m_{2}>(n-1) / 2$.

The following estimate holds uniformly with respect to $\nu \in \mathbb{R}^{2} \backslash(0,0)$ for any $m_{2} \in \mathbb{Z}_{+}, m_{2}>(n-1) / 4$. From (2.43), (2.42), and from the Cauchy inequality we have

$$
\left|\omega_{0}(x)\right|^{2} \leq c_{5}\left(\left\|\omega_{0}\right\|_{2}^{2}+\left\|\left(\Delta_{\nu, I} \otimes \mathrm{id}\right) \omega_{0}\right\|_{2}^{2}+\left\|\left(\Delta_{\nu, I} \otimes \Delta_{N}^{m_{2}}\right) \omega_{0}\right\|_{2}^{2}\right) .
$$

Indeed, the following two Banach norms on the finite-dimensional space $\operatorname{Ker} \Delta_{N}^{\bullet}$

$$
\|h\|_{B}^{2}:=\max _{x \in N}|h(x)|^{2} \quad \text { and } \quad\|h\|_{2, N}^{2}
$$


are equivalent. So we get (where $x=\left(x_{1}, x_{N}\right) \in I \times N$ and $\left.I=[-1,1]\right)$ :

$$
\begin{gathered}
\left|\left(\left(\operatorname{id}_{I} \otimes \Pi_{0}^{\bullet}(N)\right) \omega_{0}\right)(x)\right|^{2} \leq\left\|\left(\Pi_{0}^{\bullet}(N) \omega_{0}\left(x_{1}, *\right)\right)\right\|_{B}^{2} \leq c_{6}\left\|\omega_{0}\left(x_{1}, *\right)\right\|_{2, N}^{2}, \\
\left\|\omega_{0}\left(x_{1}, *\right)\right\|_{2, N}^{2} \leq 2 \sup _{x_{2}}\left|\left(G_{I}(\nu)\right)_{x_{1}, x_{2}}\right|^{2} \cdot\left\|\left(\Delta_{\nu, I} \otimes \mathrm{id}\right) \omega_{0}\right\|_{2, M}^{2} \leq 2 c_{2}^{2}\left\|\left(\Delta_{\nu, I} \otimes \mathrm{id}\right) \omega_{0}\right\|_{2}^{2} .
\end{gathered}
$$

The following estimate is obtained by the similar method:

$$
\begin{aligned}
\left|\left(\left(G_{I}(\nu) \otimes G_{N}^{m_{2}}\right)\left(\Delta_{\nu, I} \otimes \Delta_{N}^{m_{2}}\right) \omega_{0}\right)\left(x_{1}, x_{N}\right)\right|^{2} \leq \\
\quad \leq 2 c_{2}^{2} \sup _{y_{1}}\left\|\left(G_{N}^{m_{2}}\right)_{y_{1}, *}\right\|_{2, N}^{2} \cdot\left\|\left(\Delta_{\nu, I} \otimes \Delta_{N}^{m_{2}}\right) \omega_{0}\right\|_{2, M}^{2} .
\end{aligned}
$$

Hence the estimate (2.44) holds for $\omega_{0}$ (even without the first term on the right in (2.44)), as follows from (2.43), (2.45), (2.46), and (2.47).

Since $\Delta_{\nu}=\mathrm{id}_{I} \otimes \Delta_{N}+\Delta_{\nu}(I) \otimes \mathrm{id}_{N}$ and since $\Delta_{N}$ and $\Delta_{\nu, I}$ are nonnegative selfadjoint operators, we have for $m_{2} \in \mathbb{Z}_{+}$:

$$
\left\|\left(\Delta_{\nu, I} \otimes \Delta_{N}^{m_{2}}\right) \omega_{0}\right\|_{2} \leq\left\|\Delta_{\nu}^{m_{2}+1} \omega_{0}\right\|_{2} .
$$

The inequality (2.32) for $\omega_{0}$ follows from (2.44) and (2.48). For $\omega_{1}$ the analogous to (2.43) equality holds:

$$
\begin{aligned}
\omega_{1}=\left(\Pi_{0}^{1}\left(I_{\nu}\right) \otimes\right. & \left.\left(\operatorname{id}_{N}-\Pi_{0}^{\bullet-1}(N)\right)\right) \omega_{1}+\left(\left(\operatorname{id}_{I}-\Pi_{0}^{1}\left(I_{\nu}\right)\right) \otimes \Pi_{0}^{\bullet-1}(N)\right) \omega_{1}+ \\
& +\left(\Pi_{0}^{1}\left(I_{\nu}\right) \otimes \Pi_{0}^{\bullet-1}(N)\right) \omega_{1}+\left(G_{I}(\nu) \otimes G_{N}^{m_{2}}\right)\left(\Delta_{\nu, I} \otimes \Delta_{N}^{m_{2}} \omega_{1}\right),
\end{aligned}
$$

where $\Pi_{0}^{1}\left(I_{\nu}\right)$ is the projection operator of $\left(D R^{1}(I)\right)_{2}$ onto the one-dimensional space $c \cdot d x_{1}$ and $G_{I}(\nu)_{1}$ is the Green function for the Laplacian $\Delta_{\nu, I}$ on $D R^{1}\left(I_{\nu}\right)$ (with the Dirichlet boundary conditions on $\partial I=\{-1,1\}$ and with the $\nu$-transmission boundary conditions at 0 ). The kernel $G_{I}(\nu)_{1}$ is continuous on $\bar{Q}_{r_{1}} \times \bar{Q}_{r_{2}}$ because it can be written in a form similar to (2.40). It is written through the Green function $g_{1}$ of $\Delta_{I}$ on $D R^{1}(I)$ with the Dirichlet boundary conditions on $\partial I$ where the kernel $\left(g_{1}\right)_{x_{1}, x_{2}}$ of $g_{1}$ is continuous on $I \times I$. Hence the second term on the right in (2.49) is estimated similarly to (2.45) and to (2.46). The kernel $\Pi_{0}^{1}\left(I_{\nu}\right)_{x_{1}, x_{2}}$ is expressed in a form analogous to (2.40) through the kernel $2^{-1} d x_{1} \otimes d x_{2}$ on $I \times I$ (corresponding to $\left.\Pi_{0}^{1}\left(I_{1,1}\right)\right)$. So the kernel of $\Pi_{0}^{1}\left(I_{\nu}\right)$ is continuous on $\bar{Q}_{r_{1}} \times \bar{Q}_{r_{2}}$, and it satisfies the estimate (2.42) (with the upper bound $c$ ). The first and the third terms in (2.49) are estimated as follows:

$$
\begin{gathered}
\left|\left(\Pi^{1}\left(I_{\nu}\right) \otimes\left(\operatorname{id}_{N}-\Pi_{0}^{\bullet-1}(N)\right)\right) \omega_{1}\left(x_{1}, x_{N}\right)\right|^{2} \leq 2 c^{2} \sup _{y_{1}}\left\|\left(G_{N}^{m_{2}}\right)_{y_{1}, *}\right\|_{2, N}^{2}\left\|\left(\operatorname{id} \otimes \Delta_{N}^{m_{2}}\right) \omega_{1}\right\|_{2, M}^{2}, \\
\left|\left(\Pi^{1}\left(I_{\nu}\right) \otimes \Pi_{0}^{\bullet-1}(N)\right) \omega_{1}(x)\right|^{2} \leq 2 c^{2} c_{6}\left\|\omega_{1}\right\|_{2, M}^{2} .
\end{gathered}
$$

Hence the estimate (2.44) holds uniformly with respect to $\nu \in \mathbb{R}^{2} \backslash(0,0)$ for any $m \in \mathbb{Z}_{+}, m \geq m_{0}:=1+\min \left\{k \in \mathbb{Z}_{+}, 4 k \geq n\right\}$. Thus Proposition 2.8 is proved. 
2.3. Actions of the homomorphisms of identifications on the determinant. Proof of Lemma 2.3. The most simple method to compute the action of $g_{\nu *}$ on $\operatorname{Det}(M, N, Z)^{26}$ is to obtain the expression for the action of $v_{\nu *}^{c}$ on the determinant line $\operatorname{Det} C \cdot(X, V, W)$ (1.54), induced by the identifications of the corresponding cochain complexes $v_{\nu}^{c}=v_{\nu \nu_{0}}^{c}: C^{\bullet}\left(X_{\nu_{0}}, V\right) \rightarrow C^{\bullet}\left(X_{\nu}, V\right)$ (where $v_{\nu}^{c}\left(c_{1}, c_{2}\right):=$ $\left(c_{1},\left(k_{\nu} / k_{\nu_{0}}\right) c_{2}\right)$ for $\left.\nu, \nu_{0} \in U(2.3)\right)$, and then to use Proposition 2.10 below. The action of $v_{\nu *}^{c}$ is defined by identifications $\psi_{\nu}$ and $\psi_{\nu_{0}}$, where $\psi_{\nu}: \operatorname{det} C^{\bullet}\left(X_{\nu}, V\right) \vec{\sim}$ $\operatorname{Det} C \cdot(X, V, W)$ are defined by the exact sequence in the bottom row of the diagram (1.59). The following diagram of the identifications is commutative:

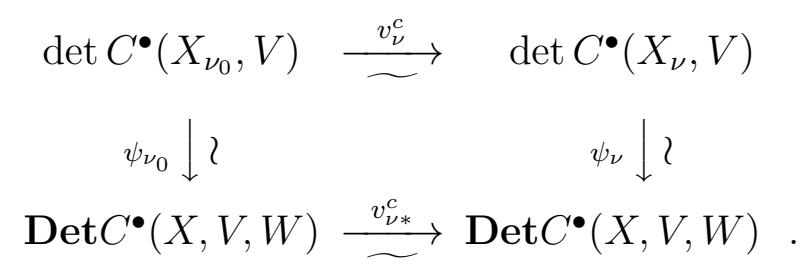

Proposition 2.10. Under the conditions of Lemma 2.3, the equality holds:

$$
g_{\nu *}=v_{\nu *}^{c} .
$$

The proof of the equality (2.50) is done just after the end of the proof of Lemma 2.3. The expression for the action of $v_{\nu *}^{c}$ on the determinant line can be obtained as follows. Let $\nu \in U$ and let $j$ be the natural inclusion $j: \oplus_{k=1,2} C^{\bullet}\left(X_{k}, W \oplus V_{k}\right) \rightarrow C^{\bullet}\left(X_{\nu}, V\right)$. Then $v_{\nu}^{c}$ acts on $C^{\bullet}\left(X_{1}, W \cup V_{1}\right)$ as the identity operator and it acts on $C^{\bullet}\left(X_{2}, W \cup V_{2}\right)$ as the operator $\left(k_{\nu} / k_{\nu_{0}}\right)$ id. Proposition 2.7 claims that the identification $\psi_{\nu}$ is an isometry between the combinatorial norm on $\operatorname{det} C^{\bullet}\left(X_{\nu}, V\right)$ and the triple tensor product of the combinatorial norms on the components of $\operatorname{Det} C^{\bullet}(X, W, V)$. It is enough to compute the action of $v_{\nu *}^{c}$ on the component $\operatorname{det} C^{\bullet}(W)$ of the tensor product $\operatorname{Det} C^{\bullet}(X, W, V)$. The inclusion $\rho_{\nu, c}: C^{\bullet}(W) \hookrightarrow C^{\bullet}\left(X_{\nu}, V\right)$ (defined by (1.63)) is an isometry onto orthogonal complement to $\operatorname{Im} j$ and $r_{\nu, c} \rho_{\nu, c}=\mathrm{id}$ on $C^{\bullet}(W)$. So the action of $v_{\nu}^{c}$ on this orthogonal complement $(\operatorname{Im} j)^{\perp}$ (identified with $C^{\bullet}(W)$ by $r_{\nu_{0}, c}$ and by $r_{\nu, c}$ ) can be expressed as the composition

$$
\begin{gathered}
m \in C^{\bullet}(W) \underset{\rho_{\nu_{0}, c}}{\longrightarrow}\left(\left(\beta_{0}, \alpha_{0}\right) / \sqrt{\alpha_{0}^{2}+\beta_{0}^{2}}\right) m \underset{v_{\nu}^{c}}{\longrightarrow}\left(\left(\beta_{0}, \alpha_{0}\left(k_{\nu} / k_{\nu_{0}}\right)\right) / \sqrt{\alpha_{0}^{2}+\beta_{0}^{2}}\right) m= \\
=\left(\left(1, k_{\nu}\right) / \sqrt{1+k_{\nu_{0}}^{2}}\right) m \underset{r_{\nu, c}}{\longrightarrow}\left(\sqrt{1+k_{\nu}^{2}} / \sqrt{1+k_{\nu_{0}}^{2}}\right) m \in C^{\bullet}(W) .
\end{gathered}
$$

${ }^{26}$ This action is multiplying by a nonzero factor. 
(Here, the signs are written for positive $\beta$ and $\beta_{0} \cdot{ }^{27}$ ) The expression for $v_{\nu *}^{c}$ follows from (2.51) and from the assertion that $v_{\nu}^{c}$ acts on $C^{\bullet}\left(X_{2}, W \cup V_{2}\right)$ as $\left(k_{\nu} / k_{\nu_{0}}\right)$ id. Namely

$$
v_{\nu *}^{c} l=\left(k_{\nu} / k_{\nu_{0}}\right)^{-\chi\left(M_{2}, N \cup Z_{2}\right)}\left(\left(1+k_{\nu}^{2}\right) /\left(1+k_{\nu_{0}}^{2}\right)\right)^{-\chi(N) / 2} l .
$$

for $l \in \operatorname{Det} C^{\bullet}(X, V, W)$. It follows from (2.52) that the equality holds (for $\left.l \neq 0\right)$ :

$$
\partial_{\gamma} \log \left\|v_{\nu *}^{c} l\right\|^{2}=-2 \chi\left(M_{2}, N \cup Z_{2}\right) \partial_{\gamma} \log \left(k_{\nu}\right)-2 \chi(N)\left(1+k_{\nu}^{-2}\right)^{-1} \partial_{\gamma} \log \left(k_{\nu}\right)
$$

Proposition 2.10 claims that the same identity holds also for the action of $g_{\nu *}$ on $\operatorname{Det}(M, N, Z)$.

The right side in the formula (2.19) (i.e., in the assertion of Lemma 2.3) is defined in analytic terms while the right side in (2.53) is defined in topological terms. Each $b_{2, j}\left(M_{\nu_{0}}, Z\right)$ on the right in (2.19) is the sum of integrals over $M_{2}$ and over $N$ of the locally defined densities, according to Theorem 3.2. So it is enough to compute (in topological terms) the expression on the right in (2.19) in the case of a mirror-symmetric $\widetilde{M}=M_{2} \cup_{N} M_{2}$ with a mirror-symmetric metric $g_{\widetilde{M}}$ (which is a direct product metric near $\partial \widetilde{M}$ and near $N$ ) and with mirror-symmetric boundary conditions on the connected components of $\partial \widetilde{M}$. In this case, the expression in (2.19) is the same as for a general $M$ (if the piece $M_{2}$ of $M,\left.g_{M}\right|_{T M_{2}}$, and the boundary conditions on $\partial M \cap M_{2}$ are the same as in the mirror-symmetric case on each piece $M_{2}$ of $\widetilde{M}$ ). It is supposed from now on in the proof of Lemma 2.3 that $M$ and all the data on $M$ are mirror-symmetric relative to $N$. In this case the kernel $E_{t, x, y}^{\bullet}(\nu)$ $\left(\nu \in \mathbb{R}^{2} \backslash(0,0)\right)$ of the operator $\exp \left(-t \Delta_{\nu}^{\bullet}\right)$ with the Dirichlet boundary conditions on $Z=Z_{2} \cup Z_{2} \subset \partial M$ and with the Neumann conditions on $\partial M \backslash N$ is expressed through the fundamental solution $E_{t, x, y}^{\bullet}$ for $\partial_{t}+\Delta^{\bullet}$ on $D R^{\bullet}(M, Z$ ) (with the same boundary conditions on $\partial M)^{28}$ as follows ${ }^{29}$ :

$$
\begin{gathered}
E_{t, x, y}^{\bullet}(\nu)=E_{t, x, y}^{\bullet}+\left(\left(\alpha^{2}-\beta^{2}\right) /\left(\alpha^{2}+\beta^{2}\right)\right)\left(\sigma_{1}^{*} E^{\bullet}\right)_{t, x, y} \text { for } x \in M_{2}, y \in M_{2} \\
E_{t, x, y}^{\bullet}(\nu)=\left(2 \alpha \beta / \alpha^{2}+\beta^{2}\right) E_{t, x, y}^{\bullet} \text { for } x \in M_{1}, y \in M_{2}
\end{gathered}
$$

Note that the kernel $\left(E_{t}+\sigma_{1}^{*} E_{t}\right)_{x, y}=: E_{t, x, y}^{N e u}$ for $x, y \in M_{2}$ is the fundamental solution for $\partial_{t}+\Delta_{M_{2}}^{\bullet}$, where $\Delta_{M_{2}}^{\bullet}$ is the Laplacian on $D R^{\bullet}\left(M_{2}, Z_{2}\right)$ with the Neumann boundary conditions on $N$ and the kernel $\left(E_{t}-\sigma_{1}^{*} E_{t}\right)_{x, y}=: E_{t, x, y}^{D i r}$ is the kernel of

\footnotetext{
${ }^{27}$ The signs are not important for the transformations of the norm on the determinant line under the actions of $v_{\nu *}^{c}$.

${ }^{28} \mathrm{It}$ is proved in Proposition 1.1 that $\Delta^{\bullet}$ on $D R^{\bullet}(M, Z)$ for $M$ obtained by gluing two pieces, $M=M_{1} \cup_{N} M_{2}$, has the same eigenvalues (including their multiplicities) and eigenforms as $\Delta_{1,1}$ in $D R^{\bullet}\left(M_{1,1}, Z\right)$. The analogous assertion is true for the operators $\exp \left(-t \Delta_{1,1}^{\bullet}\right)$ and $\exp \left(-t \Delta^{\bullet}\right)$ in $\left(D R^{\bullet}(M)\right)_{2}$ and for their kernels.

${ }^{29}$ These formulas are analogous to $(2.40)$.
} 
$\exp \left(-t \Delta_{M_{2}, N}^{\bullet}\right)$, where $\Delta_{M_{2}, N}^{\bullet}$ is the Laplacian on $D R^{\bullet}\left(M_{2}, N \cup Z_{2}\right)$ i.e., with the Dirichlet boundary conditions on $N$. It follows from (2.54) that the alternating sum of zero-order terms (in the asymptotic expansions of the traces of the heat equation operator relative to $t \rightarrow+0$ ) on the right in (2.19) can be represented in the following form (where $\left.m_{\nu_{0}}:=2^{-1}\left(1-k_{\nu_{0}}^{-2}\right) /\left(1+k_{\nu_{0}}^{-2}\right)\right)$ :

$$
\begin{aligned}
& \sum(-1)^{j} b_{2, j}\left(M_{\nu_{0}}, Z\right)=\sum(-1)^{j} \int_{M_{2}} \operatorname{tr}\left(E_{t, x, x}^{j}\left(\nu_{0}\right)\right)^{0}= \\
= & 2^{-1} \sum(-1)^{j} \int_{M} \operatorname{tr}\left(E_{t, x, x}^{j}\right)^{0}+m_{\nu_{0}} \sum(-1)^{j} \int_{M_{2}}\left(\operatorname{tr}\left(E_{t, x, x}^{j, N e u}\right)^{0}-\operatorname{tr}\left(E_{t, x, x}^{j, D i r}\right)^{0}\right)= \\
= & 2^{-1} \chi(M, Z)+m_{\nu_{0}}\left(\chi\left(M_{2}, Z_{2}\right)-\chi\left(M_{2}, Z_{2} \cup N\right)\right)=\chi\left(M_{2}, Z_{2} \cup N\right)+\left(1+k_{\nu_{0}}^{-2}\right)^{-1} \chi(N) .
\end{aligned}
$$

Hence the expression on the right in (2.53) is equal to the right side of (2.19), and the assertion of Lemma 2.3 follows from Proposition 2.10. The zero superscripts in (2.56) denote the densities on $M_{j}, N, \partial M$, corresponding to the constant terms (i.e., the $t^{0}$-coefficients) in the asymptotic expansions as $t \rightarrow+0$ for $\operatorname{Tr}\left(p_{j} \exp \left(-t \Delta^{\bullet}\right)\right)$, where $\Delta^{\bullet}$ is the Laplacian with appropriate boundary conditions. In $(2.56) \int_{M_{j}} \operatorname{tr}(.)^{0}$ denotes the sum of the integrals over $M_{j}, N$, and over $\partial M_{j} \backslash N$ of the corresponding densities. We use the following equalities to produce (2.56):

$$
\begin{aligned}
& \sum(-1)^{j} \int_{M} \operatorname{tr}\left(E_{t, x, x}^{j}\right)^{0}=\chi(M, Z), \\
& \sum(-1)^{j} \int_{M_{2}} \operatorname{tr}\left(E_{t, x, x}^{j, N e u}\right)^{0}=\chi\left(M_{2}, Z_{2}\right), \\
& \sum(-1)^{j} \int_{M_{2}} \operatorname{tr}\left(E_{t, x, x}^{j, D i r}\right)^{0}=\chi\left(M_{2}, N \cup Z_{2}\right) .
\end{aligned}
$$

These equalities are consequences of the analogous equalities without the zero superscripts and of the existence of asymptotic expansions in powers of $t$ for the corresponding traces as $t \rightarrow+0$ ([Se2], Theorem 3, or Theorem 3.2 below).

Proof of Proposition 2.10. The identifications

$$
\operatorname{Det} C^{\bullet}(X, V, W) \stackrel{d_{c}}{\longleftarrow} \operatorname{Det}(X, V, W) \stackrel{R}{\longleftarrow} \operatorname{Det}(M, N, Z)
$$

do not depend on $\nu$ (the determinant lines in (2.58) are defined in (1.54)). So the actions of $v_{\nu *}^{c}$ on $\operatorname{Det}(X, V, W)$ and on $\operatorname{Det} C^{\bullet}(X, V, W)$ are the same (i.e., they multiply by the same number). To prove (2.50) it is enough to show that the corresponding operators on $\operatorname{Det}(M, Z, N)$ are the same (i.e., that $g_{\nu *}=v_{\nu *}^{c}$ on $\operatorname{det}(M, N, Z)$ ).

The proof of Proposition 2.10 uses the following assertion. 
Proposition 2.11. Let $\varphi:\left(F_{0}^{\bullet}, d_{F_{0}}\right) \rightarrow\left(F_{1}^{\bullet}, d_{F_{1}}\right)$ be an isomorphism of finite complexes of finite-dimensional linear spaces. Then the diagram is commutative:

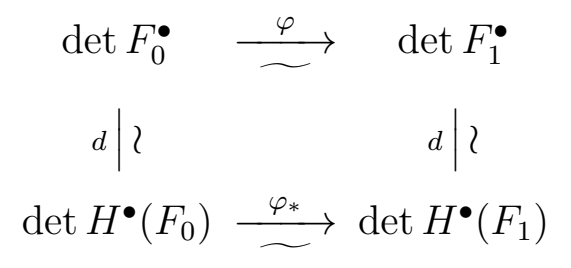

Proof. The identifications $\operatorname{det} F_{j}^{\bullet} \stackrel{d}{\sim} \operatorname{det} H^{\bullet}\left(F_{j}\right)$ are defined with the help of differentials $d=d_{F_{j}}$. Hence the commutativity of (2.59) holds.

The commutativity of the following diagram of the identifications (for $\nu$ sufficiently close to $\nu_{0}$ such that $v_{\nu}$ is an isomorphism) follows from (2.59):

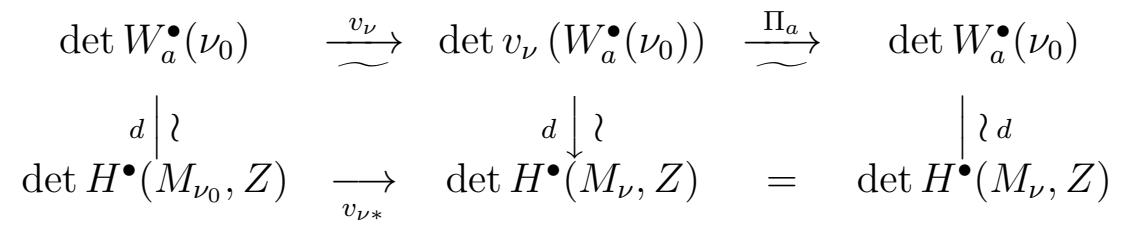

where the identification $j_{*}: H^{\bullet}\left(v_{\nu}\left(W_{a}^{\bullet}\left(\nu_{0}\right)\right)\right) \rightarrow H^{\bullet}\left(D R\left(M_{\nu}, Z\right)\right)=H^{\bullet}\left(M_{\nu}, Z\right)$ is defined by the natural inclusion $j: v_{\nu}\left(W_{a}^{\bullet}\left(\nu_{0}\right)\right) \hookrightarrow D R^{\bullet}\left(M_{\nu}, Z\right)$ of a quasi-isomorphic subcomplex. The commutativity of the left square in (2.60) follows from (2.59). The commutativity of the right square in (2.60) also follows from (2.59) because the operator induced by the projection operator $\Pi_{a}$ on $H^{\bullet}\left(D R\left(M_{\nu}, Z\right)\right)$ is the identity operator.

The commutativity of the following diagram is a consequence of the commutativity of the diagram (2.60):

$$
\begin{aligned}
& \operatorname{det} W_{a}^{\bullet}\left(\nu_{0}\right) \quad=\quad \operatorname{det} W_{a}^{\bullet}\left(\nu_{0}\right) \quad \stackrel{v_{\nu}}{\longrightarrow} \operatorname{det} v_{\nu}\left(W_{a}^{\bullet}\left(\nu_{0}\right)\right) \\
& \varphi_{\nu_{0}}(a) \mid 2 \quad \operatorname{det} H^{\bullet}\left(M_{\nu_{0}}, Z\right) \underset{v_{\nu *}}{\longrightarrow} \operatorname{det} H^{\bullet}\left(M_{\nu}, Z\right) \\
& \varphi_{\nu_{0}}^{a n}\left|2 \quad \varphi_{\nu}^{a n}\right| 2 \\
& \operatorname{Det}(M, N, Z)=\operatorname{Det}(M, N, Z) \stackrel{g_{\nu *}}{\longrightarrow} \operatorname{Det}(M, N, Z)
\end{aligned}
$$

The action of $v_{\nu *}: H^{\bullet}\left(M_{\nu_{0}}, Z\right) \rightarrow H^{\bullet}\left(M_{\nu}, Z\right)$ coincides with the combinatorial action $v_{\nu *}^{c}: H^{\bullet}\left(X_{\nu_{0}}, V\right) \rightarrow H^{\bullet}\left(X_{\nu}, V\right)$ under the identification of the cohomology $R: H^{\bullet}\left(M_{\nu}, Z\right) \rightarrow H^{\bullet}\left(X_{\nu}, V\right)$ induced by the integration $R$ of closed differential 
forms over the simplexes of $X$. Hence the commutativity of (2.60) involves also the commutativity of the diagram:

$$
\begin{aligned}
& \operatorname{det} W_{a}^{\bullet}\left(\nu_{0}\right) \stackrel{v_{\nu}}{\longrightarrow} \operatorname{det} v_{\nu}\left(W_{a}^{\bullet}\left(\nu_{0}\right)\right) \\
& \begin{array}{ccc}
d \mid r & d \mid r \\
\operatorname{det} H^{\bullet}\left(M_{\nu_{0}}, Z\right)= & \operatorname{det} H^{\bullet}\left(M_{\nu_{0}}, Z\right) \\
d_{c} \mid \prec & \underset{v_{\nu *}}{\longrightarrow} & \operatorname{det} H^{\bullet}\left(M_{\nu}, Z\right)
\end{array} \\
& \varphi_{\nu_{0}}^{c}=\varphi_{\nu_{0}}^{a n} \downarrow 2 \quad \operatorname{det} C^{\bullet}\left(X_{\nu_{0}}, V\right) \underset{v_{\nu}^{c}}{\longrightarrow} \operatorname{det} C^{\bullet}\left(X_{\nu}, V\right) \\
& \psi_{\nu_{0}}\left|2 \quad \psi_{\nu}\right| \imath \\
& \operatorname{Det} C^{\bullet}(X, V, W) \underset{v_{\nu *}^{c}}{\longrightarrow} \operatorname{Det} C^{\bullet}(X, V, W) \\
& (2.58)|2 \quad(2.58)| \imath \\
& \operatorname{Det}(M, N, Z)=\operatorname{Det}(M, N, Z) \underset{v_{\nu *}^{c}}{\longrightarrow} \operatorname{Det}(M, N, Z)
\end{aligned}
$$

The equality (2.50) follows immediately from the commutativity of the right bottom square in (2.61) and from the commutativity of (2.62). Proposition 2.10 is proved.

2.4. Analytic torsion norm on the cone of a morphism of complexes. Proof of Lemma 2.4. Lemma 2.4 is a particular case of the following assertion. Let $f$ be a morphism (2.22) of finite complexes of finite-dimensional Hilbert spaces. ${ }^{30}$ Then Cone $f$ is defined by $(2.23)$. The exact sequence of complexes: ${ }^{31}$

$$
0 \rightarrow V^{\bullet} \rightarrow \text { Cone }^{\bullet} f \underset{p}{\rightarrow} A^{\bullet}[1] \rightarrow 0
$$

(where the left arrow maps $y \in V^{\bullet}$ into $(0, y) \in$ Cone $^{\bullet} f$ and $p(x, y)=x$ for $(x, y) \in$ $\left.A^{j+1} \oplus V^{j}\right)$ defines the identification of the determinants of its cohomology:

$$
\varphi_{\text {Cone }}^{H}: \operatorname{det} H^{\bullet}(\text { Cone } f) \underset{\sim}{\sim} \operatorname{det} H^{\bullet}(V) \otimes\left(\operatorname{det} H^{\bullet}(A)\right)^{-1} \text {. }
$$

Let the Hilbert spaces Cone $^{j} f$ be the direct sums $A^{j} \oplus V^{j+1}$ of the Hilbert spaces.

Lemma 2.6. The analytic torsion norm on the determinant of the cohomology of Cone $f$ is isometric under the identification (2.64) to the tensor product of the analytic torsions norms ${ }^{32}$ :

$$
T_{0}\left(\text { Cone }^{\bullet} f\right)=T_{0}\left(V^{\bullet}\right) \otimes T_{0}\left(A^{\bullet}\right)^{-1} .
$$

\footnotetext{
${ }^{30}$ The morphism $f$ does not supposed to be a quasi-isomorphism.

${ }^{31} A^{\bullet}[1]$ is a complex with components $A[1]^{j}=A^{j+1}$ and with $d_{A[1]}=-d_{A}$. There are the canonical identifications: $\operatorname{det} A^{\bullet}[1]=\left(\operatorname{det} A^{\bullet}\right)^{-1}$ and $\operatorname{det} H^{\bullet}(A[1])=\left(\operatorname{det} H^{\bullet}(A)\right)^{-1}$.

${ }^{32}$ The analytic torsion norm on $\operatorname{det} H^{\bullet}(A[1])=\operatorname{det} H^{\bullet}(A)^{-1}$ is the dual norm $T_{0}\left(A^{\bullet}\right)^{-1}$.
} 
Remark 2.6. Let $h \in \operatorname{det} H^{\bullet}$ (Cone $f$ ) be identified by (2.64) with $h_{1} \otimes h_{2}^{-1}$ for $h_{1} \in$ $\operatorname{det} H^{\bullet}(V)$ and $h_{2} \in \operatorname{det} H^{\bullet}(A)$. Namely $\varphi_{\text {Cone }}{ }_{f} h=h_{1} \otimes h_{2}^{-1}$, where $h_{2}^{-1}$ is an element of the dual one-dimensional space $\operatorname{det} H^{\bullet}(A)^{-1}$ such that $h_{2}^{-1}\left(h_{2}\right)=1$. In this case, the equality (2.65) claims that

$$
\|h\|_{T_{0}(\text { Cone } \bullet)}^{2}=\left\|h_{1}\right\|_{T_{0}(V \bullet)}^{2} /\left\|h_{2}\right\|_{T_{0}(A \bullet)}^{2} .
$$

Remark 2.7. The identity (2.65) (Lemma 2.6) and the equality (2.66) also hold under weaker assumptions. Let the Hilbert structures on $A^{\bullet}, V^{\bullet}$, and on Cone $f$ be such that the identification

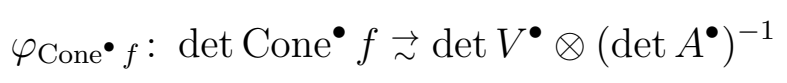

(induced by the exact sequence (2.63)) is an isometry. Then the equality (2.66) holds.

Corollary 2.7. Let $f: A^{\bullet} \rightarrow V^{\bullet}$ be a quasi-isomorphism. Then $H^{\bullet}($ Cone $f)=0$. Hence $\operatorname{det} H^{\bullet}$ (Cone $\left.f\right)$ is canonically $\mathbb{C}$ and $1 \in \mathbb{C}=\operatorname{det} H^{\bullet}($ Cone $f)$ is identified by (2.64) with $h_{1} \otimes h_{2}^{-1}$. Here, $f_{*} h_{2}=h_{1}$ under the identification induced by $f$ :

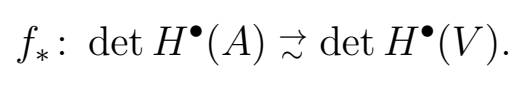

In this case, the equality (2.66) claims that

$$
\|1\|_{T_{0}(\text { Cone } f)}^{2}=\left\|h_{1}\right\|_{T_{0}\left(V^{\bullet}\right)}^{2} /\left\|h_{2}\right\|_{T_{0}\left(A^{\bullet}\right)}^{2}=T_{0}\left(V^{\bullet}\right) / T_{0}\left(A^{\bullet}\right)
$$

where $T_{0}\left(V^{\bullet}\right) / T_{0}\left(A^{\bullet}\right)$ is the ratio of the two norms on the same determinant line (since $\operatorname{det} H^{\bullet}(V)$ and $\operatorname{det} H^{\bullet}(A)$ are identified by (2.68)).

The equality (2.69) is the assertion of Lemma 2.4 .

Proof of Lemma 2.6. The identification (2.67) is an isometry of norms on the determinant lines. Let $u$ be a nonzero element of $\operatorname{det}$ Cone ${ }^{\bullet} f$ and let

$$
\varphi_{\text {Cone }}{ }_{f} u=u_{1} \otimes u_{2}^{-1}
$$

where $u_{1} \in \operatorname{det} V^{\bullet}$ and $u_{2} \in \operatorname{det} A^{\bullet}$. Let $h, h_{1}, h_{2}$ be the images of $u, u_{1}, u_{2}$ under the identifications (defined by the differentials of the corresponding complexes):

$$
\begin{aligned}
& \operatorname{det} \text { Cone }^{\bullet} f \underline{d_{\text {Cone }} f} \operatorname{det} H^{\bullet}(\text { Cone } f) \text {, }
\end{aligned}
$$

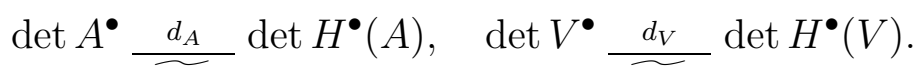

Then by the definition of $\varphi^{H}{ }_{\text {Cone }}{ }_{f}$ we have $\varphi^{H}{ }_{\text {Cone }}{ }_{f} h=h_{1} \otimes h_{2}^{-1}$.

The analytic torsion norm on the determinant of the cohomology of a finitedimensional complex is the norm, corresponding to the $L_{2}$-norm on the determinant 
of this complex defined by the Hilbert structures on its components. Hence the equalities hold:

$$
\begin{gathered}
\|h\|_{T_{0}\left(\mathrm{Cone}^{\bullet} f\right)}^{2}=\|u\|_{\operatorname{det}\left(\mathrm{Cone}^{\bullet} f\right)}^{2}, \\
\left\|h_{1}\right\|_{T_{0}\left(V^{\bullet}\right)}^{2}=\left\|u_{1}\right\|_{\operatorname{det}\left(V^{\bullet}\right)}^{2}, \quad\left\|h_{2}\right\|_{T_{0}\left(A^{\bullet}\right)}^{2}=\left\|u_{2}\right\|_{\operatorname{det}\left(A^{\bullet}\right)}^{2} .
\end{gathered}
$$

Since the identification (2.67) is an isometry, we see that the equality

$$
\|h\|_{T_{0}(\text { Cone } f)}^{2}=\left\|h_{1}\right\|_{T_{0}(V \bullet)}^{2} /\left\|h_{2}\right\|_{T_{0}(A \bullet)}^{2}
$$

follows from (2.71) and from (2.70).

The equality (2.24) in Lemma 2.4 is a particular case of (2.72) (by Corollary 2.7) corresponding to the case of a quasi-isomorphism $f$. Lemma 2.4 is proved.

2.5. Variation formula for norms of morphisms of identifications. Proof of Lemma 2.1. The assertion (2.14) of Lemma 2.1 can be deduced from the definition of $g_{\nu}$ and from the following proposition.

Proposition 2.12. There is a neighborhood $U_{\nu_{0}}, \nu_{0} \in U_{\nu_{0}} \subset \mathbb{R}^{2} \backslash(0,0)$, such that a family of finite rank projection operators $\Pi_{a}^{j}(\nu):=\Pi_{j}(\nu ; a)$ in $\left(D R^{j}(M)\right)_{2}$ is smooth on $U_{\nu_{0}} \ni \nu$.

Its proof follows just after the proof of Lemma 2.1. Let $l \in \operatorname{det} W_{a}^{\bullet}\left(\nu_{0}\right), l \neq 0$, and let $l=\otimes_{j} l_{j}^{(-1)^{j+1}}$, where $l_{j} \in \operatorname{det} W_{a}^{j}\left(\nu_{0}\right), l_{j} \neq 0$. Then we have

$$
\log \left\|g_{\nu} l\right\|_{\operatorname{det} W_{a}^{\bullet}(\nu)}^{2}=\sum(-1)^{j+1} \log \left\|g_{\nu} l_{j}\right\|_{\operatorname{det} W_{a}^{j}(\nu)}^{2}
$$

Proposition 2.13. For every $j$ the following equality holds (under the conditions of Lemma 2.1):

$$
\left.\partial_{\gamma} \log \left\|g_{\nu} l_{j}\right\|_{\operatorname{det} W_{a}^{j}(\nu)}^{2}\right|_{\gamma=0}=\left.2 \partial_{\gamma} \log \left(k_{\nu}\right)\right|_{\gamma=0} \operatorname{Tr}\left(p_{2} \Pi_{a}^{j}\left(\nu_{0}\right)\right) .
$$

Thus the assertion (2.14) of Lemma 2.1 is a consequence of (2.74) and of (2.73). Proof of Proposition 2.13. It is enough to prove the equality (2.74) in the case when $\left\|l_{j}\right\|_{\operatorname{det} W_{a}^{*}\left(\nu_{0}\right)}^{2}=1$, i.e., when $l=e_{1} \wedge \ldots \wedge e_{m}$, where $\left\{e_{i}\right\}$ is an orthonormal basis in $W_{a}^{\bullet}\left(\nu_{0}\right)\left(\nu_{0}=\nu(0)\right)$. In this case, we have

$$
\left.\partial_{\gamma} \log \left\|g_{\nu} l\right\|_{\operatorname{det} W_{a}^{j}(\nu)}^{2}\right|_{\gamma=0}=\left.\operatorname{tr}\left(\partial_{\gamma}\left(a_{i j}(\nu)\right)\right)\right|_{\gamma=0},
$$

where $a(\nu)=\left(a_{i j}(\nu)\right)$ is a matrix of scalar products in $\left(D R^{j}\left(\bar{M}_{1}\right) \oplus D R^{j}\left(\bar{M}_{2}\right), g_{M}\right)$ of the images of the basis elements

$$
a_{i j}(\nu):=<g_{\nu} e_{i}, g_{\nu} e_{j}>
$$


The formula $(2.75)$ is deduced as follows. Since $\left\|g_{\nu} l\right\|_{\operatorname{det} W_{a}^{j}(\nu)}^{2}=\operatorname{det} a(\nu)$, we have

$$
\left.\partial_{\gamma} \log \operatorname{det} a(\nu)\right|_{\gamma=0}=\left.\operatorname{tr}\left(\partial_{\gamma} a \cdot a^{-1}(\nu)\right)\right|_{\gamma=0}=\left.\operatorname{tr}\left(\partial_{\gamma} a\right)\right|_{\gamma=0} .
$$

The family of the operators $\Pi_{a}^{j}(\nu)$ is smooth in $\nu$ for $\nu \in U_{\nu_{0}}$ (Proposition 2.12). Hence the operator $\partial_{\gamma} \Pi_{a}^{j}(\nu)$ exists. Since $\Pi_{a}^{j}(\nu)$ are projection operators, we have

$$
\Pi_{a}^{2}=\Pi_{a}, \quad \partial_{\gamma} \Pi_{a} \cdot \Pi_{a}=\left(i d-\Pi_{a}\right) \partial_{\gamma} \Pi_{a} .
$$

So $e_{i}$ are orthogonal to $\left.\partial_{\gamma} \Pi_{a}\right|_{\gamma=0} e_{j}$ and we get

$$
\begin{aligned}
& \left.\partial_{\gamma} a_{i j}(\nu)\right|_{\gamma=0}=\partial_{\gamma}<\Pi_{a} g_{\nu} e_{i}, \Pi_{a} g_{\nu} e_{j}>\left.\right|_{\gamma=0}=<\partial_{\gamma}\left(\Pi_{a} g_{\nu}\right) e_{i}, e_{j}>\left.\right|_{\gamma=0}+ \\
& \quad+<e_{i}, \partial_{\gamma}\left(\Pi_{a} g_{\nu}\right) e_{j}>\left.\right|_{\gamma=0}=\left.\partial_{\gamma}\left(\log k_{\nu}\right)\right|_{\gamma=0}\left(<p_{2} e_{i}, e_{j}>+<e_{i}, p_{2} e_{j}>\right) .
\end{aligned}
$$

Since $\left\{e_{i}\right\}$ is an orthonormal basis in $W_{a}^{\bullet}\left(\nu_{0}\right)$, we have

$$
\operatorname{tr}\left(<p_{2} e_{i}, e_{j}>\right)=\operatorname{tr}\left(p_{2} \Pi_{a}^{j}\left(\nu_{0}\right)\right)
$$

Hence (2.74) follows from (2.75), (2.76), and (2.77). Thus Proposition 2.13 and Lemma 2.1 are proved.

Proof of Proposition 2.12. Let $U_{\nu_{0}} \subset \mathbb{R}^{2} \backslash(0,0)$ be the set of $\nu \in \mathbb{R}^{2} \backslash(0,0)$ such that the Laplacians $\Delta_{\nu, j}$ on $D R^{j}\left(M_{\nu}, Z\right.$ ) (for all $j$ ) have no eigenvalues $\lambda$ from $(a-\varepsilon, a+2 \varepsilon) \subset \mathbb{R}_{+}$(where $\varepsilon>0$ is small enough). Then for $\nu \in U_{\nu_{0}}$ the projection operator $\Pi_{a}^{j}(\nu)$ from $\left(D R^{j}(M)\right)_{2}$ onto a linear space $W_{a}^{j}(\nu)$ (spanned by the eigenforms of $\Delta_{\nu, j}$ with the eigenvalues $\lambda$ from $[0, a]$ ) is equal to the contour integral

$$
\Pi_{a}^{j}(\nu)=i / 2 \pi \int_{\Gamma_{a+\varepsilon}} G_{\lambda}^{j}(\nu) d \lambda
$$

where $G_{\lambda}^{j}(\nu)=\left(\Delta_{\nu, j}-\lambda\right)^{-1}$ is the resolvent for the Laplacian $\Delta_{\nu, j}$ and $\Gamma_{a+\varepsilon}$ is a circle $\Gamma_{a+\varepsilon}=\{\lambda:|\lambda|=a+\varepsilon\}$ oriented opposite to the clockwise. For $\lambda \in \Gamma_{a+\varepsilon}$ and $\nu \in U_{\nu_{0}}$ the operators $G_{\lambda}^{j}(\nu)$ form a smooth in $(\lambda, \nu)$ family of bounded operators in $\left(D R^{j}(M)\right)_{2}$. (It is an immediate consequence of Proposition 3.1 below. Indeed, $\operatorname{Spec}\left(\Delta_{\nu, j}\right)$ is discrete and it is a subset of $\mathbb{R}_{+} \cup 0$, according to Theorem 3.1. Thus if $(a-\varepsilon, a+2 \varepsilon) \cap \operatorname{Spec}\left(\Delta_{\nu, j}\right)=\emptyset$ then $\Gamma_{a+\varepsilon} \cap \operatorname{Spec}\left(\Delta_{\nu, j}\right)=\emptyset$ and $G_{\lambda}^{j}(\nu)$ form a smooth in $\nu \in U_{\nu_{0}}$ and in $\lambda \in \Gamma_{a+\varepsilon}$ family of bounded in $\left(D R^{j}(M)\right)_{2}$ operators by Proposition 3.1.) Proposition 2.13 is proved.

2.6. Variation formula for the scalar analytic torsion. Proof of Lemma 2.2. First the lemma is proved in the case when $a>0$ is less than $4^{-1} \lambda_{1}\left(\nu_{0}\right)$ (where $\lambda_{1}(\nu)$ is the minimal positive eigenvalue of the Laplacian $\Delta_{\nu}$ on $\left.\left(\oplus_{j} D R^{j}\left(M_{\nu}, Z\right), g_{M}\right)\right)$. Let $U_{1}(a)$ be a neighborhood of $\nu_{0}, \nu_{0} \in U_{1}(a) \subset U(2.3)$, such that for $\nu \in U_{1}(a)$ we have $a<2^{-1} \lambda_{1}(\nu)$. (Such a neighborhood exists according to Theorem 3.1 and to Proposition 3.1.) 
Let $\nu(\gamma)$ be a smooth local map $\left(\mathbb{R}^{1}, 0\right) \rightarrow\left(U(a), \nu_{0}\right)$.

Proposition 2.14. For $t>0$ the following variation formula holds ${ }^{33}$ :

$$
\begin{aligned}
\left.\partial_{\gamma} \sum(-1)^{j} j \operatorname{Tr} \exp \left(-t \Delta_{\nu, j}\right)\right|_{\gamma=0} & = \\
& =\left.2 \partial_{\gamma} \log \left(k_{\nu}\right)\right|_{\gamma=0} \sum(-1)^{j}(-t \partial / \partial t) \operatorname{Tr}\left(p_{1} \exp \left(-t \Delta_{\nu_{0}, j}\right)\right),
\end{aligned}
$$

where $k_{\nu}:=\alpha / \beta$ for $\nu=(\alpha, \beta) \in U$ (i.e., for $\left.\alpha \beta \neq 0\right)$.

Proposition 2.15. Let $\operatorname{Re} s>(\operatorname{dim} M) / 2$ and let $0<a<\lambda_{1}\left(\nu_{0}\right)$. Then the following equalities hold:

$$
\begin{gathered}
\left.\partial_{\gamma}\left[\Gamma(s)^{-1} \int_{0}^{\infty} t^{s-1} \sum(-1)^{j} j\left(\operatorname{Tr} \exp \left(-t \Delta_{\nu, j}\right)-\operatorname{dim} \operatorname{Ker} \Delta_{\nu, j}\right) d t\right]\right|_{\gamma=0}= \\
=\Gamma(s)^{-1} \int_{0}^{\infty} t^{s} \sum(-1)^{j}\left(-\partial_{t}\right) \operatorname{Tr}\left(p_{1}\left(\exp \left(-t \Delta_{\nu_{0}, j}\right)-\Pi_{a}^{j}\left(\nu_{0}\right)\right)\right) d t= \\
=\left.2 \partial_{\gamma} \log \left(k_{\nu}\right)\right|_{\gamma=0}(s / \Gamma(s)) \sum(-1)^{j} \int_{0}^{\infty} t^{s-1} \operatorname{Tr}\left(p_{1}\left(\exp \left(-t \Delta_{\nu_{0}, j}\right)-\Pi_{a}^{j}\left(\nu_{0}\right)\right)\right) d t \\
2.79)
\end{gathered}
$$

Proof of Proposition 2.15. To produce the second row of (2.79) from (2.78), it is enough to prove ([Bo], II.26, Proposition 7$)$ that for $\operatorname{Re} s>(\operatorname{dim} M) / 2$ the integral

$$
\int_{0}^{\infty} t^{s}\left(-\partial_{t}\right) \operatorname{Tr}\left(p_{1} \exp \left(-t \Delta_{\nu, j}\right)\right) d t=\int_{0}^{\infty} t^{s} \operatorname{Tr}\left(p_{1} \Delta_{\nu, j} \exp \left(-t \Delta_{\nu, j}\right)\right) d t
$$

converges uniformly in $\nu$ for $\nu$ from a small neighborhood $U_{\nu_{0}}$ of $\nu_{0}$ and to prove the convergence of the integral

$$
\int_{0}^{\infty} t^{s-1} \operatorname{Tr}\left(\exp \left(-t \Delta_{\nu_{0}, j}\right)-\operatorname{dim} \operatorname{Ker} \Delta_{\nu_{0}, j}\right) d t
$$

together with the uniform convergence in $\nu$ for an arbitrary $\nu_{1} \in U_{\nu_{0}}$ (as $\left.\nu \rightarrow \nu_{1}\right)$ of the function

$$
t^{s+1} \sum(-1)^{j} \operatorname{Tr}\left(p_{1} \Delta_{\nu, j} \exp \left(-t \Delta_{\nu, t}\right)\right) \rightarrow t^{s+1} \sum(-1)^{j} \operatorname{Tr}\left(p_{1} \Delta_{\nu_{1}, j} \exp \left(-t \Delta_{\nu_{1}, t}\right)\right)
$$

for $t$ from any closed finite interval $t \in I \subset(0,+\infty)$. (To apply the theorem from [Bo], quoted above, it is useful first to do the transformation $\mathbb{R}_{+} \ni t \rightarrow h=\log t \in \mathbb{R}$, $d t \rightarrow t d h$.

\footnotetext{
${ }^{33}$ The operator $\exp \left(-t \Delta_{\nu, j}\right)$ acts from $\left(D R^{j}(M)\right)_{2}$ into the domain $D\left(\Delta_{\nu, j}\right)$ of $\Delta_{\nu, j}$ defined by (1.27). The operators $\exp \left(-t \Delta_{\nu, j}\right)$ and $p_{1} \exp \left(-t \Delta_{\nu, j}\right)$ are of trace class. Their traces are equal to the integrals over the diagonal of the traces of their kernels restricted to the diagonals (by Proposition 3.8).
} 
The following estimates are satisfied

$$
\begin{gathered}
\left|\operatorname{Tr}\left(p_{1} \Delta_{\nu, j} \exp \left(-t \Delta_{\nu, j}\right)\right)\right| \leq \operatorname{Tr}\left(\Delta_{\nu, j} \exp \left(-t \Delta_{\nu, j}\right)\right) \leq \\
\leq\left\|\Delta_{\nu, j} \exp \left(-t \Delta_{\nu, j} / 2\right)\right\|_{2}\left(\operatorname{Tr}\left(\exp \left(-t \Delta_{\nu, j} / 2\right)-\operatorname{dim} \operatorname{Ker} \Delta_{\nu, j}\right)\right) \\
\left\|\Delta_{\nu, j} \exp \left(-t \Delta_{\nu, j} / 2\right)\right\|_{2} \leq \max _{\lambda \geq 0}(\lambda \exp (-t \lambda / 2))=2 /(t e)
\end{gathered}
$$

(where $\|\cdot\|_{2}$ are the operator norms in $\left.\left(D R^{j}(M)\right)_{2}\right)$. The first estimate in (2.83) follows from the Mercer theorem. (The applying of this theorem here is similar to its applying in the proofs of Propositions 3.8 and 3.9 below.)

Let $t_{0}$ be any positive number. Then for $t \geq 2 t_{0}$ and for $\nu$ sufficiently close to $\nu_{0}$ we have the following uniform with respect to $\nu$ estimate

$$
\operatorname{Tr} \exp \left(-t \Delta_{\nu, j} / 2\right)-\operatorname{dim} W_{a}^{j}(\nu)<C \exp \left(-c_{1} t\right)
$$

where $C$ and $c_{1}$ are positive constants. Indeed, according to Theorem 3.2, for any $t_{0}>0$ there is a constant $L>0$ (depending on $t_{0}$ ) such that the inequality

$$
\operatorname{Tr} \exp \left(-t_{0} \Delta_{\nu, j} / 2\right) \leq L
$$

holds uniformly with respect to $\nu \in \mathbb{R}^{2} \backslash(0,0)$. So for all $\nu \in U$ sufficiently close to $\nu_{0}$ and such that $\lambda_{1}(\nu)>2 a$, the estimate (2.85) is true for $t \geq 2 t_{0}$ with $C=L$ and $c_{1}=a / 4$ :

$$
\operatorname{Tr} \exp \left(-t \Delta_{\nu, j} / 2\right)-\operatorname{dim} W_{a}^{j}(\nu) \leq L \exp (-t a / 4)
$$

The uniform convergence (with respect to $\nu$ ) of the integral (2.80) for Re $s>$ $(\operatorname{dim} M) / 2$ follows from the asymptotic expansion (2.15) in powers of $t$ as $t \rightarrow+0$ and from the estimates $(2.83),(2.84)$, and (2.85). The convergence of the integral (2.81) for $\operatorname{Re} s>(\operatorname{dim} M) / 2$ follows from $(2.85)$ and from the existence of the asymptotic expansion of the trace as $t \rightarrow+0$ (by Theorem 3.2):

$$
\begin{aligned}
\operatorname{Tr} \exp \left(-t \Delta_{\nu_{0}, j}\right)=f_{-\operatorname{dim} M} t^{-(\operatorname{dim} M) / 2}+f_{1-\operatorname{dim} M} t^{(1-\operatorname{dim} M) / 2} & +\cdots+ \\
& +f_{k} t^{k / 2}+O\left(t^{(k+1) / 2}\right),
\end{aligned}
$$

where $k \in \mathbb{Z}_{+}$and $f_{i}:=f_{i}(\nu ; j)$ are smooth in $\nu \in \mathbb{R}^{2} \backslash(0,0)$. This asymptotic expansion (2.87) is differentiable with respect to $\nu$, according to Proposition 3.2.

The uniform convergence of (2.82) for $t \in I \subset(0, \infty)$ (if $s$ is fixed and $\operatorname{Re} s>$ $(\operatorname{dim} M) / 2$ ) follows from Proposition 3.8 and from the uniform convergence (with respect to $\nu$ ) of the functions of $t$

$$
t^{s+1} \partial_{t} \int_{M_{1}} \operatorname{Tr} E_{t, x, x}^{j}(\nu) \rightarrow t^{s+1} \partial_{t} \int_{M_{1}} \operatorname{tr} E_{t, x, x}^{j}\left(\nu_{1}\right)
$$

for $t \in I$. (The latter assertion follows from Proposition 3.2 and from Theorem 3.2.) 
The last equality in (2.79) is true for $\operatorname{Re} s>(\operatorname{dim} M) / 2$ according to the asymptotic expansion (2.87), to the estimate (2.86), to the absolute convergence of the integral $(2.80)$, and to the following estimates (where $\left.0<a<\lambda_{1}\left(\nu_{0}\right)\right)$ :

$$
0<\operatorname{Tr}\left(p_{1}\left(\exp \left(-t \Delta_{\nu_{0}, j}\right)-\Pi_{a}^{j}\left(\nu_{0}\right)\right)\right) \leq \operatorname{Tr}\left(\exp \left(-t \Delta_{\nu_{0}, j}\right)-\Pi_{a}^{j}\left(\nu_{0}\right)\right) .
$$

(These estimates are deduced from the Mercer theorem the same way as in the proofs of Propositions 3.8 and 3.9.) Thus Proposition 2.15 is proved.

Proposition 2.16. For $0<a<\lambda_{1}\left(\nu_{0}\right)$ the assertion of Lemma 2.1 is true, i.e., it holds:

$$
\left.\partial_{\gamma} \log T\left(M_{\nu}, Z\right)\right|_{\gamma=0}=\left.2 \partial_{\gamma} \log \left(k_{\nu}\right)\right|_{\gamma=0} \sum(-1)^{j} \operatorname{Tr}\left(p_{1}\left(\exp \left(-t \Delta_{\nu_{0}, j}\right)-\Pi_{a}^{j}\left(\nu_{0}\right)\right)\right)^{0}
$$

(The zero superscript denotes the constant coefficient in the asymptotic expansion as $t \rightarrow+0$ for the operator trace.)

Proof. The equality (2.79) claims that for $\operatorname{Re} s>(\operatorname{dim} M) / 2$ and for $0<a<$ $\lambda_{1}\left(\nu_{0}\right) / 4$ we have

$$
\begin{aligned}
& \left.\partial_{\gamma}\left(\sum(-1)^{j} j \zeta_{\nu, j}(s)\right)\right|_{\gamma=0}= \\
& \quad=\left.2 \partial_{\gamma} \log \left(k_{\nu}\right)\right|_{\gamma=0}(s / \Gamma(s)) \sum(-1)^{j} \int_{0}^{\infty} t^{s-1} \operatorname{Tr}\left(p_{1}\left(\exp \left(-t \Delta_{\nu_{0}, j}\right)-\Pi_{a}^{j}\left(\nu_{0}\right)\right)\right) d t .
\end{aligned}
$$

The final expressions in (2.90) and (2.79) are analytic functions of $s$ for $\operatorname{Re} s>$ $(\operatorname{dim} M) / 2$ according to $(2.88),(2.86)$, and to $(2.15)$. The meromorphic continuation to the whole complex plane $\mathbb{C} \ni s$ of this function of $s$ can be produced with the help of the asymptotic expansion (2.15) (or of the expansion (2.91) below for $\left.\operatorname{Tr}\left(p_{1}\left(\exp \left(-t \Delta_{\nu_{0}, j}\right)-\Pi_{a}^{j}\left(\nu_{0}\right)\right)\right)\right)$ using the estimates (2.88) and (2.86). Let the asymptotic expansion as $t \rightarrow+0$ for the trace of the operator below be as follows $(n:=\operatorname{dim} M)$ :

$$
\operatorname{Tr}\left(p_{1}\left(\exp \left(-t \Delta_{\nu_{0}, j}\right)-\Pi_{a}^{j}\left(\nu_{0}\right)\right)\right)=q_{-n} t^{-n / 2}+\ldots+q_{0} t^{0}+\hat{q}_{1} t^{1 / 2}+\ldots+\hat{q}_{k} t^{k / 2}+r_{k, j}(t),
$$

where $r_{k, j}(t)$ is $O\left(t^{(k+1) / 2}\right)$ as $t \rightarrow+0$ and $r_{k, j}(t)$ is a $C^{[k / 2]}$-smooth function of $t \in[0,1]{ }^{34}$ Then the analytic continuation to $\operatorname{Re} s>-(k+1) / 2$ of the integral on

\footnotetext{
${ }^{34}$ This asymptotic expansion exists and is differentiable with respect to $t$ by Proposition 3.2.
} 
the right in (2.90) is given by the expression

$$
\begin{aligned}
& \int_{0}^{\infty} t^{s-1} \operatorname{Tr}\left(p_{1}\left(\exp \left(-t \Delta_{\nu_{0}, j}\right)-\Pi_{a}^{j}\left(\nu_{0}\right)\right)\right) d t= \\
& =q_{-n} /(-n / 2+s)+q_{-n+1} /(-n / 2+1 / 2-s)+\cdots+q_{0} / s+\hat{q}_{1} /(1 / 2+s)+\ldots \\
& \quad+\hat{q}_{k} /(k / 2+s)+\int_{0}^{1} t^{s-1} r_{k, j}(t) d t+\int_{1}^{\infty} t^{s-1} \operatorname{Tr}\left(p_{1}\left(\exp \left(-t \Delta_{\nu_{0}, j}\right)-\Pi_{a}^{j}\left(\nu_{0}\right)\right)\right) d t \\
&
\end{aligned}
$$

The latter integral in (2.92) is an analytic function on the whole complex place $\mathbb{C} \in s$ (according to (2.88) and (2.86)). The integral of $r_{k, j}$ in (2.92) is an analytic function of $s$ for $\operatorname{Re} s>-(k+1) / 2$. The asymptotic expansions $(2.87)$ for $\operatorname{Tr} \exp \left(-t \Delta_{\nu, j}\right)$ can be differentiated with respect to $\nu$ according to Proposition 3.2. They provide us with the analytic continuation of $\sum(-1)^{j} j \zeta_{\nu, j}(s)$ to $\operatorname{Re} s>-(k+1) / 2$ as follows:

$$
\begin{aligned}
& \sum(-1)^{j} j \zeta_{\nu, j}(s)=\Gamma(s)^{-1}\left(F_{-n} /(-n / 2+s)+\cdots+F_{k} /(k / 2+s)+\right. \\
& \left.+\int_{0}^{1} t^{s-1} m_{k, \nu}(t) d t+\int_{1}^{\infty} t^{s-1} \sum(-1)^{j} j \operatorname{Tr}\left(\exp \left(-t \Delta_{\nu, j}\right)-\operatorname{dim} \operatorname{Ker} \Delta_{\nu, j}\right) d t\right),
\end{aligned}
$$

where $F_{k}:=\sum_{j}(-1)^{j} j\left(f_{k}(\nu, j)-\delta_{0, k} \operatorname{dim} \operatorname{Ker} \Delta_{\nu, j}\right)$ and the functions $m_{k, \nu}(t)$ are

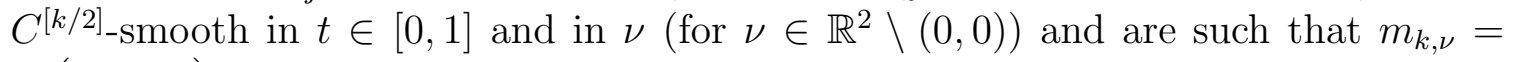
$O\left(t^{(k+1) / 2}\right)$ uniformly with respect to $\nu$ as $t \rightarrow+0$.

The latter integral in $(2.93)$ is an analytic function of $s \in \mathbb{C}$. We obtain its derivative with respect to $\gamma$ taking into account (2.78), (2.86), and (2.88):

$$
\begin{aligned}
&\left.\partial_{\gamma} \int_{1}^{\infty} t^{s-1} \sum_{j}(-1)^{j} j \operatorname{Tr}\left(\exp \left(-t \Delta_{\nu, j}\right)-\operatorname{dim} \operatorname{Ker} \Delta_{\nu, j}\right) d t\right|_{\gamma=0}= \\
&=\left.2 \partial_{\gamma} \log \left(k_{\nu}\right)\right|_{\gamma=0}\left\{s \int_{1}^{\infty} t^{s-1} \sum_{j}(-1)^{j} \operatorname{Tr}\left(p_{1}\left(\exp \left(-t \Delta_{\nu_{0}, j}\right)-\Pi_{a}^{j}\left(\nu_{0}\right)\right)\right) d t+\right. \\
&\left.\quad+\sum_{j}(-1)^{j} \operatorname{Tr}\left(p_{1}\left(\exp \left(-\Delta_{\nu_{0}, j}\right)-\Pi_{a}^{j}\left(\nu_{0}\right)\right)\right)\right\},
\end{aligned}
$$

where $0<a<\lambda_{1}\left(\nu_{0}\right) .{ }^{35}$ Using (2.87), (2.93), and (2.92), we get the equalities (where $q_{k}\left(j, \nu_{0}\right):=q_{k}$ for $k \leq 0, q_{k}\left(j, \nu_{0}\right):=\hat{q}_{k}$ for $k>0$ and $q_{k}, \hat{q}_{k}$ are from $\left.(2.91)\right)$ :

$$
\begin{aligned}
\left.\partial_{\gamma} F_{l}\right|_{\gamma=0} & =\left.2 \partial_{\gamma} \log \left(k_{\nu}\right)\right|_{\gamma=0}(-l / 2) \sum(-1)^{j} q_{l}\left(j, \nu_{0}\right), \\
\left.\partial_{\gamma} m_{k, \nu}(t)\right|_{\gamma=0} & =\left.2 \partial_{\gamma} \log \left(k_{\nu}\right)\right|_{\gamma=0}(-t) \partial_{t} \sum_{j}(-1)^{j} r_{k, j}(t) .
\end{aligned}
$$

\footnotetext{
${ }^{35}$ For such $a$ the operator $\Pi_{a}^{j}\left(\nu_{0}\right)$ is the projection operator from $\left(D R^{j}(M)\right)_{2}$ onto the space of harmonic forms $\operatorname{Ker}\left(\Delta_{\nu_{0}, j}\right)$.
} 
Hence the equality (2.90) holds on the whole complex plane $\mathbb{C} \ni s$. In particular, we obtain

$$
\left.\partial_{\gamma} \log T\left(M_{\nu}, Z\right)\right|_{\gamma=0}=\left.2 \partial_{\gamma} \log \left(k_{\nu}\right)\right|_{\gamma=0} \sum(-1)^{j} q_{0}\left(j, \nu_{0}\right)
$$

Thus Proposition 2.16 is proved.

Remark 2.8. A consequence of (2.94) is as follows. For any smooth local map $\nu(\gamma)$ : $\left(\mathbb{R}^{1}, 0\right) \rightarrow(U, 0)$ (where $U$ is defined by $(2.3)$ ) the identity $\partial_{\gamma} F_{0} \equiv 0$ holds according to (2.94). Hence the function on $U$

$$
F_{0}(\nu)=\sum(-1)^{j} j\left(f_{0}(\nu, j)-\operatorname{dim} \operatorname{Ker} \Delta_{\nu, j}\right)
$$

is independent of $\nu$. The dimension of $\operatorname{Ker} \Delta_{\nu, j}$ is independent of $\nu$ for $\nu \in U$ as it follows from the cohomology exact sequence in the top row of (2.27). Indeed, for $\nu \in U$ the dimension of $\operatorname{Im} \partial_{D}\left(\right.$ where $\partial_{D}: H^{i}(N, \mathbb{C}) \rightarrow \oplus_{k=1,2} H^{i+1}\left(M_{k}, Z_{k} \cup N ; \mathbb{C}\right.$ ) is a differential in this exact sequence) is independent of $\nu$ because for different $\nu=\nu_{0}$ and $\nu_{1}$ from $U$ the maps $\partial_{D, k}(\nu): H^{i}(N, \mathbb{C}) \rightarrow H^{i+1}\left(M_{k}, N \cup Z_{k} ; \mathbb{C}\right)$ for fixed $k=1,2$ (and for a fixed $i$ ) differ by the nonzero scalar constant factor (depending on $\nu_{0}$ and $\left.\nu_{1}\right)$. Hence $\sum(-1)^{j} j f_{0}(\nu, j)$ is a constant function on $U$.

Proof of Proposition 2.14. Let $E_{t, x, y}^{j}(\nu)$ (where $t>0$ ) be the heat kernel of the operator $\exp \left(-t \Delta_{\nu, j}\right)$. By the Duhamel principle, a variation in $\nu$ of $E_{t, x, y}^{\bullet}(\nu)$ can be written as follows. Let $\left(E_{t_{1}, x_{1}, *}^{\bullet}\left(\nu_{1}\right), E_{t_{2}, *, y}^{\bullet}(\nu)\right)_{M}=: \int_{M} E_{t_{1}, x_{1}, z}^{\bullet}\left(\nu_{1}\right) \wedge *_{z} E_{t_{2}, z, x_{2}}^{\bullet}(\nu)$ be a scalar product (1.23) (with respect to the variable $z$ ). We have

$$
\begin{aligned}
& E_{t, x, y}^{\bullet}(\nu)-E_{t, x, y}^{\bullet}\left(\nu_{0}\right)=\lim _{\varepsilon \rightarrow+0} \int_{\varepsilon}^{t-\varepsilon} d \tau \partial / d \tau\left(E_{\tau, x, *}^{\bullet}(\nu), E_{t-\tau, *, y}^{\bullet}\left(\nu_{0}\right)\right)= \\
& =\lim _{\varepsilon \rightarrow+0} \int_{\varepsilon}^{t-\varepsilon} d \tau\left[\left(-\Delta_{*} E_{\tau, x, *}^{\bullet}(\nu), E_{t-\tau, *, y}^{\bullet}\left(\nu_{0}\right)\right)+\left(E_{\tau, x, *}^{\bullet}(\nu), \Delta_{*} E_{t-\tau, *, y}^{\bullet}\left(\nu_{0}\right)\right)\right] .
\end{aligned}
$$

Stokes' formula claims that for any two smooth forms $\omega_{1}, \omega_{2} \in D R^{j}\left(\bar{M}_{1}\right)$ on a manifold $M_{1}$ with boundary we have

$$
\left(d \omega_{1}, \omega_{2}\right)_{M_{1}}-\left(\omega_{1}, \delta \omega_{2}\right)_{M_{1}}=\left(r \omega_{1}, A \omega_{2}\right)_{\partial M_{1}}:=\int_{\partial M_{1}} r \omega_{1} \wedge *_{\partial M_{1}} A \omega_{2},
$$

where the density $r \omega_{1} \wedge *_{\partial M} A \omega_{2}$ on $\partial M_{1}=: N$ and the operators $r$ and $A$ are defined as follows. Let any local orientations be chosen on $\left.T M\right|_{N}$ and on $T N$. Then the following forms on $N$ are locally defined:

$$
r \omega_{1}:=\left[N: M_{1}\right] i_{N, M_{1}}^{*} \omega_{1}, \quad A \omega_{2}:=*_{N}^{-1} i_{N, M_{1}}^{*} *_{M_{1}} \omega_{2},
$$

where $i_{N, M_{1}}^{*}: D R^{\bullet}\left(\bar{M}_{1}\right) \rightarrow D R^{\bullet}(N)$ is the geometrical restriction to $N$ (and $\left[N: M_{1}\right]=$ 1 if $N$ is locally oriented as $\partial M_{1}$ and $\left[N: M_{1}\right]=-1$ in the opposite case). The locally defined form $r \omega_{1} \wedge *_{\partial M_{1}} A \omega_{2}$ is a globally defined density on $N=\partial M^{36}$. So the

\footnotetext{
${ }^{36}$ It does not depend on a local orientation on $T M$ and if a local orientation on $T N$ changes to the opposite then this local form changes its sign.
} 
equality (2.95) can be written in the form

$$
\begin{array}{r}
E_{t, x, y}^{\bullet}(\nu)-E_{t, x, y}^{\bullet}\left(\nu_{0}\right)=\lim _{\varepsilon \rightarrow+0} \int_{\varepsilon}^{t-\varepsilon} d \tau \sum_{k=1,2}\left(\left[\left(-(r \delta)_{*} E_{\tau, x, *}^{\bullet}(\nu), A_{*} E_{t-\tau, *, y}^{\bullet}\left(\nu_{0}\right)\right)_{\partial M_{k}}+\right.\right. \\
\left.+\left(A_{*} E_{\tau, x, *}^{\bullet}(\nu),(r \delta)_{*} E_{t-\tau, *, y}^{\bullet}\left(\nu_{0}\right)\right)_{\partial M_{k}}\right]+ \\
\left.\left.-\left(r_{*} E_{\tau, x, *}^{\bullet}(\nu),(A d)_{*} E_{\tau, x, *}^{\bullet}(\nu), r_{*} E_{t-\tau, *, y}^{\bullet}\left(\nu_{0}\right)\right)_{\partial M_{k}}\right]\right),
\end{array}
$$

where $r=r_{k}, A=A_{k}$ are the corresponding operators for pairs $\left(M_{k}, \partial M_{k}\right)$.

Let any local orientations be chosen on $\left.T M\right|_{N}$ and on $T N$. Then the conditions (1.27) for the domain $D\left(\Delta_{\nu}^{\bullet}\right)$ claim that for the kernels $E^{\bullet}(\nu)$ and $E^{\bullet}\left(\nu_{1}\right)$ (where $\nu=(\alpha, \beta)$ and $\nu_{1}=\left(\alpha_{1}, \beta_{1}\right)$ are from $\left.\mathbb{R}^{2} \backslash(0,0)\right)$ the following equalities hold on $N$ :

$$
\begin{aligned}
\alpha r_{z}\left(M_{1}, N\right) \circ E_{t, x, z}^{\bullet}(\nu) & =-\beta r_{z}\left(M_{2}, N\right) \circ E_{t, x, z}^{\bullet}(\nu), \\
\beta A_{z}\left(M_{1}, N\right) \circ E_{t, x, z}^{\bullet}(\nu) & =\alpha A_{z}\left(M_{2}, N\right) \circ E_{t, x, z}^{\bullet}(\nu), \\
\alpha r_{z}\left(M_{1}, N\right) \circ \delta_{z} E_{t, x, z}^{\bullet}(\nu) & =-\beta r_{z}\left(M_{2}, N\right) \circ \delta_{z} E_{t, x, z}^{\bullet}(\nu), \\
\beta A_{z}\left(M_{1}, N\right) \circ d_{z} E_{t, x, z}^{\bullet}(\nu) & =\alpha A_{z}\left(M_{2}, N\right) \circ d_{z} E_{t, x, z}^{\bullet}(\nu),
\end{aligned}
$$

The analogous equalities hold for $E_{t}^{\bullet}\left(\nu_{1}\right)$ (where $(\alpha, \beta)$ are replaced by $\left(\alpha_{1}, \beta_{1}\right)$ ). Hence the equality (2.98) for $\nu \in U(a) \subset U$ can be written in the form

$$
\begin{aligned}
E_{t, x, y}^{\bullet}(\nu)-E_{t, x, y}^{\bullet}\left(\nu_{0}\right) & = \\
=\lim _{\varepsilon \rightarrow+0} \int_{\varepsilon}^{t-\tau} d \tau[- & \left(1-\left(k_{\nu} / k_{\nu_{0}}\right)\right)\left((r \delta)_{*} E_{\tau, x, *}^{\bullet}(\nu), A_{*} E_{t-\tau, *, y}^{\bullet}\left(\nu_{0}\right)\right)_{\partial M_{1}}+ \\
+ & \left(1-\left(k_{\nu_{0}} / k_{\nu}\right)\right)\left(A_{*} E_{\tau, x, *}^{\bullet}(\nu),(r \delta)_{*} E_{t-\tau, *, y}^{\bullet}\left(\nu_{0}\right)\right)_{\partial M_{1}}+ \\
+ & \left(1-\left(k_{\nu_{0}} / k_{\nu}\right)\right)\left(A_{*} d_{*} E_{\tau, x, *}^{\bullet}(\nu), r_{*} E_{t-\tau, *, y}^{\bullet}\left(\nu_{0}\right)\right)_{\partial M_{1}}- \\
& \left.\quad-\left(1-\left(k_{\nu} / k_{\nu_{0}}\right)\right)\left(r_{*} E_{\tau, x, *}^{\bullet}(\nu), A_{*} d_{*} E_{t-\tau, *, y}^{\bullet}\left(\nu_{0}\right)\right)_{\partial M_{1}}\right] .
\end{aligned}
$$

The kernel $E_{t, x, y}^{\bullet}(\nu)$ is smooth in $\nu \in \mathbb{R}^{2} \backslash(0,0)$ as a smooth double form on $\bar{M}_{r_{1}} \times \bar{M}_{r_{2}}$ (where the limit values on $N \subset \partial \bar{M}_{r}$ are taken from the side of $M_{r}$ ) according to Proposition 3.2. So we can conclude from (2.100) that

$$
\begin{array}{r}
\left.\partial_{\gamma} E_{t, x, y}^{\bullet}(\nu)\right|_{\gamma=0}=\left.\partial_{\gamma} \log \left(k_{\nu}\right)\right|_{\gamma=0} \lim _{\varepsilon \rightarrow+0} \int_{\varepsilon}^{t-\varepsilon} d \tau\left[\left((r \delta)_{*} E_{\tau, x, *}^{\bullet}\left(\nu_{0}\right), A_{*} E_{t-\tau, *, y}^{\bullet}\left(\nu_{0}\right)\right)_{\partial M_{1}}+\right. \\
+\left(A_{*} E_{\tau, x, *}^{\bullet}\left(\nu_{0}\right),(r \delta)_{*} E_{t-\tau, *, y}^{\bullet}\left(\nu_{0}\right)\right)_{\partial M_{1}}+\left(A_{*} d_{*} E_{\tau, x, *}^{\bullet}\left(\nu_{0}\right), r_{*} E_{t-\tau, *, y}^{\bullet}\left(\nu_{0}\right)\right)_{\partial M_{1}}+ \\
\left.+\left(r_{*} E_{\tau, x, *}^{\bullet}\left(\nu_{0}\right), A_{*} d_{*} E_{t-\tau, *, y}^{\bullet}\left(\nu_{0}\right)\right)_{\partial M_{1}}\right], \quad(2.101)
\end{array}
$$

where the limits of the kernels are taken from the side of $M_{1}$. 
By Proposition 3.8, we have the equality

$$
\operatorname{Tr} \exp \left(-t \Delta_{\nu_{0}, j}\right)=\sum_{r=1,2} \int_{\bar{M}_{r}} i_{M_{r}}^{*} E_{t, x_{1}, x_{2}}^{j}\left(\nu_{0}\right)
$$

where the exterior product of double forms on the diagonals $i_{M_{r}}: \bar{M}_{r} \hookrightarrow \bar{M}_{r} \times \bar{M}_{r}$ are implied. that

We deduce from the semigroup property for the kernels of the operators $\exp \left(-t \Delta_{\nu_{0}, j}\right)$

$$
\begin{aligned}
& \left(Q_{1, z_{1}} E_{t_{1}, x, z_{1}}^{j}\left(\nu_{0}\right), Q_{2, z_{2}} E_{t_{2}, z_{2}, x}^{j}\left(\nu_{0}\right)\right)_{M}:= \\
& \quad=\int_{M} Q_{1, z_{1}} E_{t_{1}, x, z_{1}}^{j}\left(\nu_{0}\right) \wedge_{x} *_{x} Q_{2, z_{2}} E_{t_{2}, z_{2}, x}^{j}\left(\nu_{0}\right)=Q_{1, z_{1}} Q_{2, z_{2}} E_{t_{1}+t_{2}, z_{2}, z_{1}}^{j}\left(\nu_{0}\right),
\end{aligned}
$$

where $Q_{i, z_{i}}$ are differential operators acting on differential forms, $t_{i}>0$ and the integration is with respect to the variable $x$. We deduce the following variation formula from (2.101) using (2.102) and (2.103):

$$
\begin{array}{r}
\left.\partial_{\gamma} \operatorname{Tr} \exp \left(-t \Delta_{\nu, j}\right)\right|_{\gamma=0}=\left.2 \partial_{\gamma} \log \left(k_{\nu}\right)\right|_{\gamma=0} t\left[\left(A_{z_{1}}\left(M_{1}, N\right) d_{z_{1}} r_{z_{2}}\left(M_{1}, N\right) E_{t, z_{1}, z_{2}}^{j}\left(\nu_{0}\right)\right)_{N}+\right. \\
\left.+\left(A_{z_{1}}\left(M_{1}, N\right) r_{z_{2}}\left(M_{1}, N\right) \delta_{z_{2}} E_{t, z_{1}, z_{2}}^{j}\left(\nu_{0}\right)\right)_{N}\right], \quad(2.104)
\end{array}
$$

where the summands are the integrals of the densities on $N$ (as it is defined in (2.96) and (2.97)). For instance, ${ }^{37}$

$$
\left(A_{z_{1}} d_{z_{1}} r_{z_{2}} E_{t, z_{1}, z_{2}}^{j}\left(\nu_{0}\right)\right)_{N}:=\int\left(A_{z_{1}} d_{z_{1}} \wedge *_{z_{2}, N} r_{z_{2}} E_{t, z_{1}, z_{2}}^{j}\left(\nu_{0}\right)\right) .
$$

The local forms $F_{1}=\left(i_{M_{1}}^{*} *_{z_{2}} d_{z_{1}} d_{z_{2}} E_{t, z_{1}, z_{2}}^{\bullet}\left(\nu_{0}\right)\right)$ and $F_{2}=\left(i_{M_{1}}^{*} *_{z_{2}} \delta_{z_{1}} d_{z_{1}} E_{t, z_{1}, z_{2}}^{\bullet+1}\left(\nu_{0}\right)\right)$ are smooth on the diagonal $i_{M_{1}}: \bar{M}_{1} \hookrightarrow \bar{M}_{1} \times \bar{M}_{1}$. (The limit values on $N \subset \partial M_{1}$ of these double forms are taken from the side of $M_{1}$ and the exterior product of these double forms is implied.) The local form $i_{N}^{*} *_{N, z_{2}} A_{z_{1}} d_{z_{1}} r_{z_{2}} E_{t, z_{1}, z_{2}}\left(\nu_{0}\right)$ is a smooth density on the diagonal $N \subset \partial M_{1}, i_{N}: N \hookrightarrow N \times N$. Hence we can apply Stokes' formula (2.96) to the expression on the right in (2.104). The result transformed with the help of the equality $d_{x} E_{t, x, y}^{\bullet}(\nu)=\delta_{y} E_{t, x, y}^{\bullet+1}(\nu)$ is as follows:

$$
\begin{aligned}
\left.\partial_{\gamma} \operatorname{Tr} \exp \left(-t \Delta_{\nu, j}\right)\right|_{\gamma=0}= & \\
=\left.2 \partial_{\gamma} \log \left(k_{\nu}\right)\right|_{\gamma=0} t & {\left[-\left(\delta_{z_{1}} d_{z_{1}} E_{t, z_{1}, z_{2}}^{j}\left(\nu_{0}\right)\right)_{M_{1}}+\left(d_{z_{1}} \delta_{z_{1}} E_{t, z_{1}, z_{2}}^{j+1}\left(\nu_{0}\right)\right)_{M_{1}}+\right.} \\
& \left.+\left(d_{z_{2}} \delta_{z_{2}} E_{t, z_{1}, z_{2}}^{j}\left(\nu_{0}\right)\right)_{M_{1}}-\left(\delta_{z_{2}} d_{z_{2}} E_{t, z_{1}, z_{2}}^{j-1}\left(\nu_{0}\right)\right)_{M_{1}}\right]
\end{aligned}
$$

\footnotetext{
${ }^{37}$ We imply (but do not write) the restriction to the diagonal $N \hookrightarrow N \times N$ in (2.105).
} 
where the summands in (2.106) are the integrals of the densities of the type:

$$
\left(\delta_{z_{1}} d_{z_{1}} E_{t, z_{1}, z_{2}}^{\bullet}\left(\nu_{0}\right)\right)_{M_{1}}:=\int_{M_{1}} i_{M_{1}}^{*} *_{z_{2}} \delta_{z_{1}} d_{z_{1}} E_{t, z_{1}, z_{2}}^{\bullet}\left(\nu_{0}\right)
$$

(and the exterior product of double forms is implied in (2.107) and (2.106)).

The formula (2.78) is an immediate consequence of (2.106) if we take into account the equalities

$$
\partial_{t} E_{t, x, y}^{\bullet}=-\Delta_{x} E_{t, x, y}^{\bullet}=-\Delta_{y} E_{t, x, y}^{\bullet}, \quad E_{t, x, y}^{\bullet}=E_{t, y, x}^{\bullet}
$$

and use the formula (3.67) in Proposition 3.8. Thus Proposition 2.14 is proved. Hence Lemma 2.2 is proved in the case of $a<4^{-1} \lambda_{1}\left(\nu_{0}\right)$.

Let now $a>0$ be an arbitrary number such that $a \notin \cup_{j} \operatorname{Spec}\left(\Delta_{\nu_{0}, j}\right)$. Then for any nonzero element $l \in \operatorname{det} W_{a}^{\bullet}\left(\nu_{0}\right)$ we have by Lemma 2.1 that

$$
\left.\partial_{\gamma} \log \left\|g_{\nu} l\right\|_{\operatorname{det} W_{a}^{\bullet}\left(\nu_{0}\right)}^{2}\right|_{\gamma=0}=-\left.2 \partial_{\gamma} \log \left(k_{\nu}\right)\right|_{\gamma=0} \sum(-1)^{j} \operatorname{Tr}\left(p_{2} \Pi_{a}^{j}\left(\nu_{0}\right)\right),
$$

where $g_{\nu}=\Pi_{a}^{\bullet} v_{\nu}$ is defined by (2.13). We know that under the identifications (2.4) and (2.6), the analytic torsion norm $T_{0}\left(M_{\nu}, Z ; a\right)$ on $\operatorname{det} W_{a}^{\bullet}(\nu)$ transforms into the analytic torsion norm $T_{0}\left(M_{\nu}, Z\right)$ on $\operatorname{det} H^{\bullet}\left(M_{\nu}, Z\right),\left\|g_{\nu} l\right\|_{T_{0}\left(M_{\nu}, Z ; a\right)}^{2}=\left\|\left(g_{\nu} l\right)_{H}\right\|_{T_{0}\left(M_{\nu}, Z\right)}^{2}$. (Here, $\left(g_{\nu} l\right)_{H} \in \operatorname{det} H^{\bullet}\left(M_{\nu}, Z\right)$ corresponds to $g_{\nu} l$ under these identifications.) Let $\left(g_{\nu} l\right)_{H}$ be fixed. Then the analytic torsion norm of $g_{\nu} l \in \operatorname{det} W_{a}^{\bullet}(\nu)$

$$
\left\|g_{\nu} l\right\|_{T_{0}\left(M_{\nu}, Z ; a\right)}^{2}:=\left\|g_{\nu} l\right\|_{\operatorname{det} W_{a}^{\bullet}(\nu)}^{2} \cdot T\left(M_{\nu}, Z ; a\right)
$$

is independent of $a>0$. Let $\nu_{0}=\left(\alpha_{0}, \beta_{0}\right) \in U$ (i.e., $\left.\alpha_{0} \beta_{0} \neq 0\right)$. Then using (2.108), (2.89) we obtain $\left(\mu:=\left.\partial_{\gamma} \log \left(k_{\nu}\right)\right|_{\gamma=0}\right)$ :

$$
\begin{aligned}
& \left.\partial_{\gamma} \log T\left(M_{\nu}, Z ; a\right)\right|_{\gamma=0}=\left.\lim _{\varepsilon \rightarrow+0} \partial_{\gamma} \log T\left(M_{\nu}, Z ; \varepsilon\right)\right|_{\gamma=0}+ \\
& +2 \mu\left(-\lim _{\varepsilon \rightarrow+0} \sum(-1)^{j} \operatorname{Tr}\left(p_{2} \Pi_{\varepsilon}^{j}\left(\nu_{0}\right)\right)+\sum(-1)^{j} \operatorname{Tr}\left(p_{2} \Pi_{a}^{j}\left(\nu_{0}\right)\right)\right)= \\
& =2 \mu\left[\sum(-1)^{j}\left(\operatorname{Tr} p_{1} \exp \left(-t \Delta_{\nu_{0}, j}\right)\right)^{0}-\sum(-1)^{j} \operatorname{Tr} \Pi_{\varepsilon}^{j}\left(\nu_{0}\right)+\sum(-1)^{j} \operatorname{Tr}\left(p_{2} \Pi_{a}^{j}\left(\nu_{0}\right)\right)\right]
\end{aligned}
$$

Note that for arbitrary $c>0, a>0$ we have

$$
\sum(-1)^{j} \operatorname{Tr} \Pi_{c}^{j}\left(\nu_{0}\right)=\chi\left(M_{\nu_{0}} ; Z\right)=\sum(-1)^{j} \operatorname{Tr}\left(\left(p_{1}+p_{2}\right) \Pi_{a}^{j}\left(\nu_{0}\right)\right) .
$$

So the final expression in (2.109) is equal to

$$
\left.2 \partial_{\gamma} \log \left(k_{\nu}\right)\right|_{\gamma=0} \sum(-1)^{j} \operatorname{Tr}\left(p_{1}\left(\exp \left(-t \Delta_{\nu_{0}, j}\right)-\Pi_{a}^{j}\left(\nu_{0}\right)\right)\right)
$$

Thus Lemma 2.2 is proved for an arbitrary $a>0$ such that $a \notin \cup_{j} \operatorname{Spec}\left(\Delta_{\nu_{0}, j}\right)$. 
2.7. Continuity of the truncated scalar analytic torsion. Proof of Proposition 2.1. Taking into account the definition (2.11) of the truncated scalar analytic torsion $T\left(M_{\nu}, Z ; a\right)$, we see that Proposition 2.1 is a consequence of the assertion as follows. For Re $s>(\operatorname{dim} M) / 2$ the truncated $\zeta$-function (2.8) for $\Delta_{\nu, j}$ is defined by the integral ${ }^{38}$

$$
\zeta_{\nu, j}(s ; a)=\Gamma(s)^{-1} \int_{0}^{\infty} t^{s-1} \operatorname{Tr}\left(\exp \left(-t \Delta_{\nu, j}\right)\left(1-\Pi_{a}^{j}(\nu)\right)\right) d t
$$

Proposition 2.17. For $a>0$ the truncated determinant for the $\nu$-transmission interior boundary conditions

$$
\operatorname{det}\left(\Delta_{\nu}^{\bullet} ; a\right):=\exp \left(-\partial /\left.\partial s \zeta_{\nu, \bullet}(s ; a)\right|_{s=0}\right)
$$

is a continuous function of $\nu$ for $\nu \in \mathbb{R}^{2} \backslash(0,0)$ such that $a \notin \operatorname{Spec}\left(\Delta_{\nu}^{\bullet}\right)$.

Proof. Let $E_{t, x, y}^{\bullet}(\nu), t>0$, be the kernel of $\exp \left(-t \Delta_{\nu}^{\bullet}\right)$. According to Proposition 3.8 we have

$$
\operatorname{Tr} \exp \left(-t \Delta_{\nu}^{\bullet}\right)=\sum_{k=1,2} \int_{\bar{M}_{k}} \operatorname{tr}\left(*_{x_{2}} i_{k}^{*} E_{t, x_{1}, x_{2}}^{\bullet}\right),
$$

where $i: \bar{M}_{k} \hookrightarrow \bar{M}_{k} \times \bar{M}_{k}$ are the diagonal immersions. (The exterior product of the restriction to the diagonal of double forms is implied in (2.111).) Set $I=[-1,1]$. Let $I \times N \subset M$ be the inclusion of the neighborhood of $N=0 \times N$ into $M$ and let $g_{M}$ be a direct product metric on $I \times N$. Let $\Delta_{\nu ; 0}^{\bullet}$ be the Laplacian on $D R^{\bullet}(I \times N)$ with the $\nu$-transmission boundary conditions on $N=0 \times N$ and with the Dirichlet boundary conditions on $\partial I \times N$. Let $E_{t, x, y}^{\bullet}(\nu ; 0), t>0$, be the kernel of $\exp \left(-t \Delta_{\nu ; 0}^{\bullet}\right)$. The equality analogous to $(2.111)$ holds also for $\operatorname{Tr} \exp \left(-t \Delta_{\nu ; 0}^{\bullet}\right)$ (where $\bar{M}_{k}$ is replaced by $\left.Q_{k} \times N, Q_{1}:=[-1,0], Q_{2}:=[0,1]\right)$.

Let $\nu_{0} \neq(0,0)$ and let $a \notin \operatorname{Spec}\left(\Delta_{\nu_{0}}^{\bullet}\right)$ be a fixed positive number. Then from Theorem 3.2, Proposition 3.1, and from the estimate (2.86) it follows that for an arbitrary $\varepsilon>0$ there are a neighborhood $U_{0}(\varepsilon)$ of $\nu_{0}$ and $T>0$ such that for $\nu \in U_{0}(\varepsilon)$ and for $t \geq T$ the estimate holds

$$
\left|\operatorname{Tr}\left(\exp \left(-t \Delta_{\nu}^{\bullet}\right)\left(1-\Pi_{a}^{\bullet}(\nu)\right)\right)\right| \leq \varepsilon \exp (-a t / 2)
$$

To prove the continuity of $\operatorname{det}\left(\Delta_{\nu}^{\bullet}\right)$ in $\nu$ at $\nu=\nu_{0}$, it is enough to obtain the following estimate. ${ }^{39}$ For a given $b, 1>b>0$, and for an arbitrary $\varepsilon>0$ there exists

\footnotetext{
${ }^{38}$ The analytic continuation of this integral from $\operatorname{Re} s>(\operatorname{dim} M) / 2$ to the whole complex plane coincides with the meromorphic continuation of $\zeta_{\nu, j}(s ; a)$.

${ }^{39}$ The integrals $(2.110)$ for the values $\nu_{0}$ and $\nu$ of the transmission parameter have the analytic continuations from $\operatorname{Re} s>(\operatorname{dim} M) / 2$ to the whole complex plane. It follows from the estimates (2.113) and (2.112) that the difference of these integrals multiplied by $\Gamma(s)$ is an absolutely convergent integral for $\operatorname{Re} s>-1$. Hence this difference is an analytic function of $s$ for such $s$ and it is equal to the difference of the analytic continuations of the integrals (2.110) for $\nu$ and $\nu_{0}$.
} 
a neighborhood $U_{\varepsilon} \ni \nu_{0}$ such that the estimate holds for $\nu \in U_{\varepsilon},-(1-b)<s<1$ :

$$
\int_{0}^{T}\left|\operatorname{Tr}\left(\exp \left(-t \Delta_{\nu}^{\bullet}\right)\left(1-\Pi_{a}^{\bullet}(\nu)\right)\right)-\operatorname{Tr}\left(\exp \left(-t \Delta_{\nu_{0}}^{\bullet}\right)\left(1-\Pi_{a}^{\bullet}\left(\nu_{0}\right)\right)\right)\right| t^{s-1} d t<\varepsilon
$$

The spectrum $\operatorname{Spec}\left(\Delta_{\nu}^{\bullet}\right)$ is discrete and it depends continuously on $\nu$ (by Proposition 3.1). Since $a \notin \operatorname{Spec}\left(\Delta_{\nu}^{\bullet}\right)$ we see that $\operatorname{Tr} \Pi_{a}^{\bullet}(\nu)=\operatorname{Tr} \Pi_{a}^{\bullet}\left(\nu_{0}\right)\left(=\operatorname{rk} \Pi_{a}^{\bullet}\right)$ in a neighborhood of $\nu_{0}$ and that the following estimate is satisfied uniformly with respect to $s,-1 / 2<s<1$ and to $\nu$ for $\nu$ sufficiently close to $\nu_{0}$ :

$$
\int_{0}^{T}\left|\operatorname{Tr}\left(\exp \left(-t \Delta_{\nu}^{\bullet}\right) \Pi_{a}^{\bullet}(\nu)\right)-\operatorname{Tr}\left(\exp \left(-t \Delta_{\nu_{0}}^{\bullet}\right) \Pi_{a}^{\bullet}\left(\nu_{0}\right)\right)\right| t^{s-1} d t<\varepsilon / 2
$$

The inequality

$$
\int_{0}^{T}\left|\operatorname{Tr} \exp \left(-t \Delta_{\nu}^{\bullet}\right)-\operatorname{Tr} \exp \left(-t \Delta_{\nu_{0}}^{\bullet}\right)\right| t^{s-1} d t<\varepsilon / 2
$$

for $\nu$ sufficiently close to $\nu_{0}$ and for $-1 / 2<s<1$ is obtained as follows. According to Proposition 3.1, $\operatorname{Tr} \exp \left(-t \Delta_{\nu}^{\bullet}\right)$ is equal to the integral over $\cup \bar{M}_{j}$ of the density defined by the restriction to the diagonal of the corresponding kernel. So it is enough to estimate in (2.115) the integrals of the difference between the densities defined by $\exp \left(-\Delta_{\nu}^{\bullet}\right)$ and by $\exp \left(-t \Delta_{\nu_{0}}^{\bullet}\right)$ separately over a fixed neighborhood $U$ of $N=$ $0 \times N \hookrightarrow M$ and over $M \backslash U$. The estimate of the integral over $U \supset N$ is obtained with the help of the kernel $E_{t, x, y}^{\bullet}(\nu ; 0)$ of $\exp \left(-t \Delta_{\nu ; 0}^{\bullet}\right)$. Set $e_{t, x, y}^{\bullet}(\nu):=E_{t, x, y}^{\bullet}(\nu)-E_{t, x, y}^{\bullet}(\nu ; 0)$ for $x, y \in I \times N$.

Proposition 2.18. For an arbitrary $m \in \mathbb{Z}_{+}$there is a neighborhood of $\nu_{0}$ such that for all $x, y \in M_{[-1 / 2,1 / 2]}:=[-1 / 2,0] \times N \cup[0,1 / 2] \times N \hookrightarrow M_{1} \cup M_{2}$ and for $t \in(0,1]$ the estimate is satisfied uniformly with respect to $\nu \in \mathbb{R}^{2} \backslash(0,0)$

$$
\left|E_{t, x, y}^{\bullet}(\nu)\right| \leq c_{m} t^{m}
$$

(where $c_{m}$ is independent of $t$ and of $\nu$ ).

Proposition 2.19. The following estimate holds uniformly with respect to $s$ for $-(1-b)<s<1$ and to $\nu$ for $\nu$ sufficiently close to $\nu_{0}$

$$
\int_{0}^{T} t^{s-1} d t\left|\int_{M_{[-1 / 2,1 / 2]}} \operatorname{tr}\left(* i_{k}^{*} e_{t, x_{1}, x_{2}}^{\bullet}(\nu)\right)-\operatorname{tr}\left(* i_{k}^{*} e_{t, x_{1}, x_{2}}^{\bullet}\left(\nu_{0}\right)\right)\right|<\varepsilon / 4
$$


Remark 2.9. For $x, y \in[-1,1] \times N$ the equalities hold (analogous to (2.54), (2.55), $(2.40))$ :

$$
E_{t, x, y}^{\bullet}(\nu ; 0)= \begin{cases}E_{t, x, y}^{\bullet}+\left(\beta^{2}-\alpha^{2} / \beta^{2}+\alpha^{2}\right)\left(\sigma_{1}^{*} E^{\bullet}\right)_{t, x, y} & \text { for } x, y \in Q_{1} \times N \\ E_{t, x, y}^{\bullet}+\left(\alpha^{2}-\beta^{2} / \beta^{2}+\alpha^{2}\right)\left(\sigma_{1}^{*} E^{\bullet}\right)_{t, x, y} & \text { for } x, y \in Q_{2} \times N \\ \left(2 \alpha \beta / \alpha^{2}+\beta^{2}\right) E_{t, x, y}^{\bullet} & \text { for } x, y \text { from different } Q_{k} \times N .\end{cases}
$$

Here, $E_{t, x, y}^{\bullet}$ is the kernel of $\exp \left(-t \Delta^{\bullet}\right)$ on $I \times N$ with the Dirichlet boundary conditions on $\partial I \times N$ and $\sigma_{1}$ is the mirror symmetry with respect to $N=0 \times N$ acting on the variable $x$ of the kernel. So we get

$$
\int_{M_{[-1 / 2,1 / 2]}} \operatorname{tr}\left(*_{x_{2}} i_{k}^{*} e_{t, x_{1}, x_{2}}^{\bullet}(\nu)\right)=\int_{M_{[-1 / 2,1 / 2]}} \operatorname{tr}\left(*_{x_{2}} i_{k}^{*}\left(E_{t, x_{1}, x_{2}}^{\bullet}(\nu)-E_{t, x_{1}, x_{2}}^{\bullet}\right)\right) \text {. }
$$

From this equality and from the estimate (2.117) it follows that the integral over $M_{[-1 / 2,1 / 2]}$ of the difference between the kernels for $\nu$ and for $\nu_{0}$ gives the term in (2.115) which is less than $\varepsilon / 4$.

Proposition 2.20. The following estimate holds uniformly with respect to $s,-(1-$ $b)<s<1$, and to $\nu$ for $\nu$ sufficiently close to $\nu_{0}$ :

$$
\int_{0}^{T}\left|\int_{M \backslash M_{[-1 / 2,1 / 2]}} \operatorname{tr}\left(*_{x_{2}} i^{*} E_{t, x_{1}, x_{2}}^{\bullet}(\nu)\right)-\operatorname{tr}\left(*_{x_{2}} i^{*} E_{t, x_{1}, x_{2}}^{\bullet}\left(\nu_{0}\right)\right)\right| d t<\varepsilon / 4 .
$$

The estimate $(2.115)$ is a consequence of (2.117), (2.119), and (2.120). The estimate (2.113) follows from $(2.114),(2.115)$. The estimate (2.115) together with (2.112) gives us the continuity of $\Delta^{\bullet}(\nu ; a)$ in $\nu$ at $\nu_{0}$. Thus we get the proofs of Propositions 2.17 and 2.1 .

Proof of Proposition 2.18. The following equality is obtained similarly to (2.98) by using of (2.99):

$$
\begin{aligned}
e_{t, x, y}(\nu)=-\lim _{\varepsilon \rightarrow+0} \int_{\varepsilon}^{t-\varepsilon} d \tau \partial / \partial \tau \int_{\partial I \times N}\left[\left((r \delta)_{*} E_{\tau, x, *}^{\bullet}(\nu), A_{*} E_{t-\tau, *, y}^{\bullet}(\nu ; 0)\right)_{\partial I \times N}+\right. \\
\left.+\left(r_{*} E_{\tau, x, *}^{\bullet}(\nu),(A d)_{*} E_{t-\tau, *, y}^{\bullet}(\nu ; 0)\right)_{\partial I \times N}\right],
\end{aligned}
$$

where the operators $r$ and $A$ correspond to the pair $(I \times N, \partial I \times N)$. So the estimate (2.116) follows from the analogous estimates for the kernels

$$
r_{z} E_{t, x, z}^{\bullet}(\nu), \quad(r \delta)_{z} E_{t, x, z}^{\bullet}(\nu), \quad A_{z} E_{t, z, y}^{\bullet}(\nu ; 0), \quad(A d)_{z} E_{t, z, y}^{\bullet}(\nu ; 0),
$$

where $x, y \in M_{[-1 / 2,1 / 2]}$ and $z \in \partial I \times N=\{-1,1\} \times N \hookrightarrow M$. Such estimates are derived with the help of Proposition 2.5 for $\Delta_{\nu}^{\bullet}$ and $\Delta_{\nu ; 0}^{\bullet}$ as follows. 
Let $m \in \mathbb{Z}_{+}$be taken large enough. Then there is an approximate fundamental solution $P^{\bullet(m)}(\nu)$ for $\left(\partial_{t}+\Delta_{\nu, x}^{\bullet}\right)$ which is the sum of an interior term $P_{i n t}^{\bullet(m)}$ and of terms, defined near the boundaries $\partial M$ and $N$. The kernel $P^{\bullet(m)}(\nu)$ is a good approximation for $E_{t, x, y}^{\bullet}(\nu)$ for small $t>0$. Its interior term is defined as follows. For any closed Riemannian $\left(M, g_{M}\right)$ there is a locally defined parametrix $p_{t, x, y}^{\bullet(m)}$ (i.e., an approximate fundamental solution for $\left.\left(\partial_{t}+\Delta_{M}^{\bullet}\right)\right)$ such that the difference $\left(p^{\bullet(m)}-E^{\bullet}\right)_{t, x, y}$ (where $E_{t, x, y}^{\bullet}$ is the fundamental solution for $\left(\partial_{t}+\Delta_{M}^{\bullet}\right)$ ) is a $C^{\infty}$-double form for $t>0$ and such that the following estimates hold uniformly with respect to $(x, y) \in M \times M$ and to $t \in(0, T]$ :

$$
\begin{aligned}
\left|p_{t, x, y}^{\bullet(m)}-E_{t, x, y}^{\bullet}\right| & \leq c_{m} t^{-n / 2+m+1}, \\
\left|\left(\Delta_{x}^{\bullet}\right)^{k}\left(p_{t, x, y}^{\bullet(m)}-E_{t, x, y}^{\bullet}\right)\right| & \leq c_{m, k} t^{-n / 2+m+1-k} \\
\left|\left(\partial_{t}+\Delta_{M}^{\bullet}\right) p_{t, x, y}^{\bullet(m)}\right| & <c_{(m)} t^{-n / 2+m}, \\
\left|\left(\Delta_{x}^{\bullet}\right)^{k}\left(\partial_{t}+\Delta_{M}^{\bullet}\right) p_{t, x, y}^{\bullet(m)}\right| & <c_{(m, k)} t^{-n / 2+m-k}
\end{aligned}
$$

([RS], Proposition 5.3, [BGV], Theorems 2.20, 2.23, 2.26, 2.30). Such a parametrix can be represented in the following form $(n:=\operatorname{dim} M)$ :

$$
p_{t, x, y}^{\bullet(m)}=(4 \pi t)^{-n / 2} \exp \left(-d(x, y)^{2} / 4 t\right) f(d(x, y)) \sum_{i=0}^{m} t^{i} \Phi_{i}(x, y),
$$

where $d(x, y)$ is the geodesic distance between $x$ and $y, f \in C_{0}^{\infty}\left(\mathbb{R}_{+}\right), f(\tau) \equiv 1$ for $0 \leq \tau \leq \varepsilon$ and $f \equiv 0$ for $\tau>2 \varepsilon$. The injectivity radius $i\left(M, g_{M}\right)$ is supposed to be greater than $2 \varepsilon$, i.e., the exponential map $\exp _{x} B_{2 \varepsilon}$ is a diffeomorphism on its image for any $x \in M$ (where $B_{2 \varepsilon}:=\left\{\xi \in T_{x} M,|\xi| \leq 2 \varepsilon\right\}$ ). The coefficients $\Phi_{i}(x, y)$ in (2.124) are smooth double forms on $M \times M$ whose germs on the diagonal $M \hookrightarrow$ $M \times M$ are unique. The principal term $\Phi_{0}(x, y)$ is the kernel of the parallel transport in $\wedge^{\bullet} T M$ along the geodesic line $\exp _{y} \xi=x$ from $y$ into $x$ (and it is defined for $\left.d(x, y)<i\left(M, g_{M}\right)\right)$. Each $\Phi_{i}(x, y)$ is determined through $\Phi_{i-1}(x, y)$ in differential geometry terms and it is well-defined for $d(x, y)<i\left(M, g_{M}\right)([\mathrm{RS}]$, Sect. 5, [BGV], Theorem 2.26, Lemma 2.49).

Let $0 \times N \hookrightarrow I \times N \hookrightarrow M$ be a neighborhood of $N=0 \times N \hookrightarrow M$, where the metric $g_{M}$ is a direct product. The fundamental solution for $\left(\partial_{t}+\Delta_{\nu}^{\bullet}\right)$ on $I \times N$ (with the Dirichlet boundary conditions on $\partial I \times N)$ is

$$
\mathcal{P}^{\bullet}(\nu)=\sum_{i=0,1} E_{I, t}^{i}(\nu) \otimes E_{N, t}^{\bullet-i}
$$

where $E_{I, t}^{\bullet}(\nu)$ is defined by the formulas completely analogous to (2.40) and (2.118). (Here, $E_{N, t}^{\mathbf{e}}$ is the fundamental solution for $\left(\partial_{t}+\Delta_{N}^{\bullet}\right)$. The operator corresponding 
to the kernel $E_{I, t}^{i}(\nu) \otimes E_{N, t}^{\bullet-i}$ acts on $D R^{i}(I, \partial I) \otimes D R^{\bullet-i}(N)$. The kernel $E_{I, t}^{i}(\nu)$ corresponds to the Laplacian on $I$ with the Dirichlet boundary conditions on $\partial I$ and with the $\nu$-transmission boundary conditions at $0 \in I$. The term in (2.125) with $i=1$ is equal to zero for $\bullet=0$.)

The parametrix $P_{t, x, y}^{\bullet(m)}(\nu)$ for $\left(\partial_{t}+\Delta_{\nu}^{\bullet}\right)$ on $M$ is defined by

$$
P_{t, x, y}^{\bullet(m)}(\nu)=\psi \mathcal{P}_{t, x, y}^{\bullet}(\nu) \varphi+\psi_{1} p_{t, x, y}^{\bullet(m)}(1-\varphi) .
$$

Here, $\varphi=\varphi\left(y_{1}\right), \psi=\psi\left(x_{1}\right)$ (in the coordinates $\left(x_{1}, x^{\prime}\right)=x$ and $\left(y_{1}, y^{\prime}\right)=y$ of points in $I \times N), \varphi, \psi \in C_{0}^{\infty}(I, \partial I) ; \varphi, \psi \geq 0, \varphi\left(y_{1}\right) \equiv 1$ for $\left|y_{1}\right| \leq 2^{-1}+\varepsilon, \varphi\left(y_{1}\right) \equiv 0$ for $\left|y_{1}\right| \geq 5 / 8, \psi \equiv 1$ in a neighborhood of $\operatorname{supp} \varphi$, and $\psi \equiv 0$ for $\left|x_{1}\right| \geq 3 / 4$, $\psi_{1} \in C_{0}^{\infty}(M \backslash N), \psi_{1} \equiv 1$ in a neighborhood of $\operatorname{supp}(1-\varphi) \subset M \backslash M_{[-1 / 2,1 / 2]}$. Hence the parametrix $P_{t, x, y}^{\bullet(m)}$ is equal to zero for $y \in M_{[-1 / 2,1 / 2]}, x \in M \backslash([-3 / 4,3 / 4] \times N)$. The term $\psi_{1} p^{\bullet(m)}(1-\varphi)$ in $(2.126)$ is defined from now on as $P_{i n t}^{\bullet(m)}$. (In the case of $\partial M \neq \emptyset$ the terms, completely analogous to $\mathcal{P}^{\bullet}(\nu)$ for $\nu \in\{(0,1),(1,0)\}$, have to be added to $P^{\bullet(m)}$. Their supports are in $([0,1] \times \partial M)^{2} \hookrightarrow M \times M$ and $g_{M}$ is a direct product metric on $[0,1] \times \partial M$.)

Proposition 2.21. 1. The boundary condition for $P_{t, x, y}^{\bullet(m)}(\nu)$ on $N$ and on $\partial M$ and the boundary condition for $\left(\Delta_{x}^{\bullet}\right)^{k} P_{t, x, y}^{\bullet(m)}(\nu)\left(k \in \mathbb{Z}_{+}\right)$are the same as for $E_{t, x, y}^{\bullet}(\nu)$ and for $\left(\Delta_{\nu, x}^{\bullet}\right)^{k} E_{t, x, y}^{\bullet}(\nu)$. Namely $P_{t, x, y}^{\bullet(m)}(\nu)$ is a smooth in $t>0$ and in $(x, y) \in \bar{M}_{j_{1}} \times \bar{M}_{j_{2}}$ kernel, $\Delta_{\nu, x}^{k} P_{t, x, y}^{(m)}(\nu) \subset D\left(\Delta_{\nu, x}^{\bullet}\right)$ for fixed $y, t>0$, and for any $k \in \mathbb{Z}_{+} \cup 0$. Here $D\left(\Delta_{\nu, x}^{\bullet}\right) \subset D R^{\bullet}\left(M_{\nu}, Z\right)$ is the domain of definition of $\Delta_{\nu, x}^{\bullet}$ on pairs $\left(\omega_{1}, \omega_{2}\right)$ of smooth forms on $\bar{M}_{j}$. It is defined by (1.27)).

2. The following estimates (analogous to (2.123)) hold for $t \in(0, T]$ uniformly with respect to $\nu \in \mathbb{R}^{2} \backslash(0,0)$ and to $(x, y) \in \bar{M}_{j_{1}} \times \bar{M}_{j_{2}}$ (with $C_{m}, C_{m, k}$ independent of $t)$ :

$$
\begin{aligned}
& \left|\left(\partial_{t}+\Delta_{\nu, x}^{\bullet}\right) P_{t, x, y}^{\bullet(m)}(\nu)\right|<C_{m} t^{-n / 2+m}, \\
& \left|\left(\Delta_{\nu, x}^{\bullet}\right)^{k}\left(\partial_{t}+\Delta_{\nu, x}^{\bullet}\right) P_{t, x, y}^{\bullet(m)}(\nu)\right|<C_{m, k} t^{-n / 2+m-k} .
\end{aligned}
$$

The kernel $r_{t, x, y}^{\bullet(m)}(\nu):=\left(\partial_{t}+\Delta_{\nu, x}^{\bullet}\right) P_{t, x, y}^{\bullet(m)}(\nu)$ is smooth in $(x, y) \in M \times \bar{M}_{j}$ and its $C^{2 l}$-norm on $M \times \bar{M}_{j}$ is estimated through $c_{m, l} t^{-n / 2+m-l}$. For any linear differential operator $F$ of order $d=d(F)$, acting on double forms on $M \times \bar{M}_{j}$, and for any $T>0$ the kernel $F \circ r_{t, x, y}^{\bullet(m)}(\nu)$ satisfies the estimate as follows when $t \in(0, T]$. It hold uniformly with respect to $(x, y) \in M \times \bar{M}_{j}$ and to $\nu \in \mathbb{R}^{2} \backslash(0,0)$

$$
\left|F \circ r_{t, x, y}^{\bullet(m)}(\nu)\right|<c(F) t^{-(n+d) / 2+m} .
$$


3. For $0 \leq k \leq[-n / 2]+m-1$ the following condition is satisfied uniformly with respect to $\nu \in \mathbb{R}^{2} \backslash(0,0)$ and to $(x, y) \in \bar{M}_{j_{1}} \times \bar{M}_{j_{2}}$ :

$$
\lim _{t \rightarrow+0}\left(\Delta_{\nu, x}^{\bullet}\right)^{k}\left(E^{\bullet}-P^{\bullet(m)}\right)_{t, x, y}(\nu)=0
$$

Corollary 2.8. 1. For $k \in \mathbb{Z}_{+}, 0 \leq k \leq[-n / 2]+m-1$ the following estimates of $L_{2}$-norms (with respect to the variable $x$ ) of the kernel $\left(P^{\bullet(m)}(\nu)-E^{\bullet}(\nu)\right)_{t, x, y}$ hold for $t \in(0, T]$ uniformly in $y \in \cup_{j=1,2} \bar{M}_{j}$ and in $\nu \in \mathbb{R}^{2} \backslash(0,0)$, where $C_{m}$ and $C_{m, k}$ are the same as in (2.127) and (2.128):

$$
\begin{gathered}
\left\|\left(E^{\bullet}(\nu)-P^{\bullet(m)}(\nu)\right)_{t, *, y}\right\|_{2} \leq C_{m}(-n / 2+m+1)^{-1} t^{-n / 2+m+1}, \\
\left\|\Delta_{\nu, *}^{k}\left(E^{\bullet}(\nu)-P^{\bullet(m)}(\nu)\right)_{t, *, y}\right\|_{2} \leq C_{m, k}(-n / 2+m+1-k)^{-1} t^{-n / 2+m+1-k} .
\end{gathered}
$$

2. The following estimate for $E_{t, x, y}^{\bullet}(\nu)$ holds for $t \in(0, T]$ and for an arbitrary $q \in \mathbb{Z}_{+}$uniformly with respect to $\nu \in \mathbb{R}^{2} \backslash(0,0)$, to $x \in M \backslash([-3 / 4,3 / 4] \times N)$, and to $y \in M_{[-1 / 2,1 / 2]}$ :

$$
\left|E_{t, x, y}^{\bullet}(\nu)\right| \leq C_{q} t^{-n / 2+q} .
$$

It holds according to (2.131), (2.132), and (2.32). (Indeed, for such $x, y$ and for an arbitrary $m \in \mathbb{Z}_{+}$we have $E_{t, x, y}^{\bullet}(\nu)=\left(E^{\bullet}(\nu)-P^{\bullet(m)}(\nu)\right)_{t, x, y}$. Hence $m$ can be chosen large enough to get (2.133).)

The estimate (2.131) is a consequence of (2.127) and of the following equality

$$
\begin{gathered}
\left(E^{\bullet}-P^{\bullet(m)}\right)_{t, x, y}(\nu)=\lim _{\varepsilon \rightarrow+0} \int_{\varepsilon}^{t-\varepsilon} d \tau \partial_{\tau}\left(E_{t-\tau, x, *}^{\bullet}(\nu),\left(E^{\bullet}-P^{\bullet(m)}\right)_{\tau, *, y}(\nu)\right)_{M}= \\
=\lim _{\varepsilon \rightarrow+0} \int_{\varepsilon}^{t-\varepsilon} d \tau\left(E_{t-\tau, x, *}^{\bullet}(\nu),\left(\partial_{\tau}+\Delta_{\nu, *}^{\bullet}\right)\left(E^{\bullet}-P^{\bullet(m)}\right)_{\tau, *, y}(\nu)\right)_{M} \cdot
\end{gathered}
$$

This equality follows from the assertions that $\left(E^{\bullet}-P^{\bullet(m)}\right)_{t, x, y}(\nu) \rightarrow 0$ as $t \rightarrow+0$ and that $\left(E^{\bullet}-P^{\bullet}(m)\right)_{\tau, x, y}(\nu) \in D\left(\Delta_{\nu, x}^{\bullet}\right)$ with respect to the variable $x$. 
The estimate (2.131) is a consequence of (2.134), (2.127), and (2.135), since the operator $\exp \left(-t \Delta_{\nu}^{\bullet}\right)$ for $t>0$ is bounded in $\left(D R^{\bullet}(M)\right)_{2}$ and its operator norm is less or equal to one:

$$
\left\|\exp \left(-t \Delta_{\nu}^{\bullet}\right)\right\|_{2} \leq 1
$$

(This inequality follows from Theorems 3.1 and 3.2. They claim that $\Delta_{\nu}^{\bullet}$ is a nonnegative self-adjoint unbounded operator in $\left(D R^{\bullet}(M)\right)_{2}$ and that $\exp \left(-t \Delta_{\nu}^{\bullet}\right)$ is a trace class operator.)

The inequality (2.132) for $1 \leq k \leq[-n / 2]+m-1$ is a consequence of (2.127), (2.135), and of the following equality (which is a generalization of (2.134)):

$$
\begin{aligned}
\Delta_{\nu, x}^{k}\left(E^{\bullet}-\right. & \left.P^{\bullet(m)}\right)_{t, x, y}(\nu)= \\
& =\lim _{\varepsilon \rightarrow+0} \int_{\varepsilon}^{t-\varepsilon}\left(E_{t-\tau, x, *}^{\bullet}(\nu),\left(\Delta_{\nu, *}^{\bullet}\right)^{k}\left(\partial_{\tau}+\Delta_{\nu, *}\right)\left(E^{\bullet}-P^{\bullet(m)}\right)_{\tau, *, y}(\nu)\right)_{M} .
\end{aligned}
$$

This equality holds since $\Delta_{\nu, x}^{k}\left(E^{\bullet}-P^{\bullet(m)}\right)_{t, x, y}(\nu) \rightarrow 0$ as $t \rightarrow+0$ (for $0 \leq k \leq$ $[-n / 2]+m-1)$ and since $\left(\Delta_{\nu, x}^{\bullet}\right)^{k}\left(E^{\bullet}-P^{\bullet}(m)\right)_{t, x, y}(\nu) \in D\left(\Delta_{\nu, x}^{\bullet}\right)$ for fixed $y$ and $t>0$.

The proof of Proposition 2.21 is preceded by the proof of Proposition 2.18.

The estimates analogous to (2.133) (with $t \in(0, T]$ and $q \in \mathbb{Z}_{+}$) hold also for the kernel $E_{t, x, y}^{\bullet}(\nu ; 0)=\mathcal{P}_{t, x, y}^{\bullet}$ of $\exp \left(-t \Delta_{\nu ; 0}^{\bullet}\right)$, where $x \in(I \backslash[-3 / 4,3 / 4]) \times N, y \in$ $M_{[-1 / 2,1 / 2]}$. Indeed, such estimates are true $(n$ is replaced by 1$)$ for $\left(E_{I, t}^{i}(\nu)\right)_{x_{1}, y_{1}}$ with $x_{1} \in I \backslash[-3 / 4,3 / 4]$ and $y_{1} \in[-1 / 2,1 / 2]$, and the kernel $\left(E_{N}^{\bullet^{-i}}\right)_{x^{\prime}, y^{\prime}}$ is $O\left(t^{-(n-1) / 2}\right)$ for $t \in(0, T]$.

The desired estimates for $(r \delta) E^{\bullet}(\nu)$ and $A d E^{\bullet}(\nu ; 0)$ are obtained from the estimates (2.131), (2.132), and from the generalization of the inequality (2.32) as follows. Let $K$ be an arbitrary first order differential operator acting on $D R^{\bullet}\left(\bar{M}_{j}\right)$. Let $\omega \in$ $D R^{\bullet}\left(M_{\nu}, Z\right)$ obeys the conditions (2.33) with $m_{1}=1+\min \{l: 4 l \geq \operatorname{dim} M+1\}$. Then the inequality is satisfied uniformly with respect to $\omega$ and to $x \in \bar{M}_{1} \cup \bar{M}_{2}$ :

$$
|K \omega(x)|^{2}<C_{K} \sum_{i=0}^{m_{1}}\left\|\Delta_{\nu}^{i} \omega\right\|_{2}^{2},
$$

where $C_{K}>0$ is independent of $\nu \in \mathbb{R}^{2} \backslash(0,0)$. The proof of (2.136) is exactly the same as the proof of (2.32) given above except the kernel $\left(G_{I}(\nu) \otimes G_{N}^{m_{2}}\right)_{x, y}$ has to be replaced by $K_{x}\left(G_{I}(\nu) \otimes G_{N}^{m_{2}}\right)_{x, y}$. Thus Proposition 2.18 is proved.

Proof of Proposition 2.21. 1. For $x$ from a neighborhood of $N=0 \times N \hookrightarrow M$, where $\psi_{1} \equiv 0$, the parametrix $P_{t, x, *}^{\bullet(m)}(\nu)$ is equal to $\mathcal{P}_{t, x, *}^{\bullet(m)}(\nu) \varphi(*)$. So $P_{t, x, *}^{\bullet(m)}(\nu) \in$ 
$D\left(\left(\Delta_{\nu, x}^{\bullet}\right)^{k}\right)$ with respect to the variable $x$, since $\mathcal{P}_{t, x, *}^{\bullet(m)}(\nu) \in D\left(\left(\Delta_{\nu, x}^{\bullet}\right)^{k}\right)$ for $k \in \mathbb{Z}_{+}$.

2. The estimates (2.127) and (2.128) hold for the term $\psi \mathcal{P}^{\bullet}(\nu) \varphi$ of $P^{\bullet(m)}(\nu)$ with an arbitrary $m \in \mathbb{Z}_{+}$, since $\left(\partial_{t}+\Delta_{\nu, x}^{\bullet}\right) \mathcal{P}^{\bullet}(\nu)=0$ (for $\left.x \in(I \backslash \partial I) \times N\right)$ and since $\min _{x_{1} \in A} \min _{y_{1} \in \operatorname{supp} \varphi}\left(\left|x_{1}-y_{1}\right|,\left|x_{1}+y_{1}\right|\right)>\delta>0$ (where $A:=\operatorname{supp}\left(\partial_{x_{1}} \psi\right)$ and the number $\delta>0$ is fixed). For $x_{1} \in A$ and $y_{1} \in \operatorname{supp} \varphi$ the estimate (2.133) ( $n$ is replaced by 1 ) holds with an arbitrary $q \in \mathbb{Z}_{+}$for the fundamental solution $\left(E_{I, t}^{\bullet}\right)_{x, y}$ of $\left(\partial_{t}+\Delta_{I}^{\bullet}\right)$ with the Dirichlet boundary conditions on $\partial I$. The same estimate for such $x_{1}, y_{1}$ holds for the kernels $\left(\sigma_{1}^{*} E_{I, t}^{\bullet}\right)_{x_{1}, y_{1}}$ and $\left(E_{I, t}^{\bullet}(\nu)\right)_{x_{1}, y_{1}}$. So this estimate holds also for the kernel $\left(\mathcal{P}^{\bullet}(\nu)\right)_{\left(x_{1}, x^{\prime}\right),\left(y_{1}, y^{\prime}\right)}$ (defined by $\left.(2.126)\right)$, since the kernel $\left(E_{N, t}^{\bullet}\right)_{x^{\prime}, y^{\prime}}$ is $O\left(t^{-(n-1) / 2}\right)$ uniformly with respect to $\left(x^{\prime}, y^{\prime}\right)$ ([RS], Proposition 5.3, [BGV], Theorem 2.23). By the analogous reasons, for such $x_{1}, y_{1}$ the estimate (2.133) with an arbitrary $q \in \mathbb{Z}_{+}$holds for the kernel $\left(F \circ \mathcal{P}^{\bullet}(\nu)\right)_{\left(x_{1}, x^{\prime}\right),\left(y_{1}, y^{\prime}\right)}$, where $F$ is a linear differential operator of finite order $d(F)$ on $M \times M$, acting on double forms on $\bar{M}_{j_{1}} \times \bar{M}_{j_{2}}$, and $n$ in (2.133) is replaced by $n+d(F)$.

So the estimate $(2.133)$ with an arbitrary $q \in \mathbb{Z}_{+}$is satisfied by $\left(\partial_{t}+\Delta_{\nu, x}^{\bullet}\right)\left(\psi \mathcal{P}^{\bullet} \varphi\right)$ and by $\left(\Delta_{\nu, x}^{\bullet}\right)^{k}\left(\partial_{t}+\Delta_{\nu, x}^{\bullet}\right)\left(\psi \mathcal{P}^{\bullet} \varphi\right)$ with $k \in \mathbb{Z}_{+}$.

The estimates (2.127) and (2.128) hold for $\psi_{1} p^{\bullet(m)}(1-\varphi)=: P_{\text {int }}^{\bullet(m)}$, since they hold for $p_{t, x, y}^{\bullet(m)}$ and since the distance on $M$ between the closure $\bar{B}$ of $B$ (where $\left.B:=\left\{x: d_{x} \psi_{1} \neq 0\right\}\right)$ and the support $\operatorname{supp}(1-\varphi)$ is greater than a positive number $\delta$. Hence the uniform with respect to $(x, y) \in \bar{B} \times \operatorname{supp}(1-\varphi)$ estimate $(2.133)$ (with an arbitrary $q \in \mathbb{Z}_{+}$) is satisfied by $p_{t, x, y}^{\bullet(m)}$. This estimate holds also for the kernel $F p_{t, x, y}^{\bullet(m)}$, where $F$ is a linear differential operator of finite order $d(F)$, acting on the smooth kernels, defined on $M \times M$. (For instance, the function $f(d(x, y))$ in the definition (2.124) of $p_{t, x, y}^{\bullet(m)}$ can be chosen such that $f(\tau) \equiv 0$ for $\tau \geq \delta$. These estimates follow also from [RS], Proposition 5.3, estimates (5.5), and from [BGV], Theorem 2.23(2). $)^{40}$

3. The difference $\left(E^{\bullet}-P^{\bullet(m)}\right)_{t, x, y}(\nu)$ can be written as the Volterra series (analogous to $[\mathrm{BGV}], 2.4)$ :

$$
\begin{aligned}
\left(E^{\bullet}\right. & \left.-P^{\bullet(m)}\right)_{t, x, y}(\nu)= \\
& =\sum_{l \geq 1}(-t)^{l} \int_{\Delta_{l}} \int_{\left(y_{1}, \ldots, y_{l}\right) \in\left(\bar{M}_{1} \cup \bar{M}_{2}\right)^{l}} P_{\sigma_{0} t, x_{1}, y_{1}}^{\bullet(m)}(\nu) r_{\sigma_{1} t, y_{1}, y_{2}}^{(m)}(\nu) \ldots r_{\sigma_{l} t, y_{l}, y}^{(m)}(\nu),
\end{aligned}
$$

\footnotetext{
${ }^{40}$ For the sake of brievity the proof of Proposition 2.21 is written in the case of $\partial M=\emptyset$.
} 
where $\Delta_{l}=\left\{\left(\sigma_{0}, \ldots, \sigma_{l}\right): 0 \leq \sigma_{i} \leq 1, \sum \sigma_{i}=1\right\}^{41}$ and $r_{t, x, y}^{(m)}(\nu):=\left(\Delta_{\nu, x}+\partial_{t}\right) P_{t, x, y}^{\bullet(m)}(\nu)$ (a scalar product $\operatorname{tr}\left(\omega_{1} \wedge * \omega_{2}\right)$ with its values in densities on $M$ is implied in (2.137)). The assertion (2.130) follows from the convergence of the series (2.137) in the topology of uniform convergence of smooth kernels on $\bar{M}_{j_{1}} \times \bar{M}_{j_{2}}$ together with their partial derivatives of orders $\leq 2 k$ on $\bar{M}_{j_{1}} \times \bar{M}_{j_{2}}$ (i.e., in the $C^{2 k}$-topology on $\bar{M}_{j_{1}} \times \bar{M}_{j_{2}}$ ).

Indeed, the definition of $P_{t, x, y}^{\bullet(m)}(2.126)$ involves that the kernel $r_{t, x, y}^{(m)}(\nu)$ is equal to zero for $x$ from a fixed (independent of $t, \nu$, and $m$ ) neighborhood of $N=0 \times N \hookrightarrow M$ in $M$. So $r_{t, x, y}^{(m)}(\nu)$ is a smooth kernel on $M \times \bar{M}_{j}$, and the inequalities (2.129) claim that the $C^{2 k}$-norm of $r_{t, x, y}^{(m)}(\nu)$ is $O\left(t^{-n / 2+m-k}\right)$ for $t \in(0, T]$ uniformly with respect to $\nu \in \mathbb{R}^{2} \backslash(0,0)$. It is $O(t)$ for $0 \leq k \leq[-n / 2]+m-1$, and the series (2.137) is convergent in the $C^{2 k}$-topology for such $k$, since the volume of $\Delta_{l}$ is $(l !)^{-1}$ and since the following assertion is true. For any $T>0$ the parametrix $P_{t, x, y}^{\bullet(m)}(\nu)$ defines a family of bounded operators from the space of smooth forms $D R^{\bullet}(M)$ (equiped with a $C^{2 k}$-norm) into the space $\oplus_{j=1,2} D R^{\bullet}\left(\bar{M}_{j}\right)$ (equiped with a $C^{2 k}$-norm on $\left.D R^{\bullet}\left(\bar{M}_{j}\right)\right)$. These operators are bounded uniformly with respect to $t \in(0, T]$ and to $\nu \in \mathbb{R}^{2} \backslash(0,0)$. This assertion for the operators, corresponding to $P_{i n t, t}^{\bullet(m)}$, is proved in $[\mathrm{BGV}]$, Lemma 2.49. It is also true for the operators corresponding to $\psi \mathcal{P}_{t}^{\bullet(m)}(\nu) \varphi$. Indeed, it holds for the operators $\exp \left(-t \Delta_{N}^{\bullet}\right)$ in $D R^{\bullet}(N)$ (equiped with a $C^{2 k}$-norm) and for the operators $\psi \exp \left(-t \Delta_{I}^{\bullet}\right) \varphi$ in $D R^{\bullet}(I)$ equiped with a $C^{2 k}$-norm. (Here $\Delta_{I}^{\bullet}$ is defined on forms with the Dirichlet boundary conditions on $\partial I$.) It holds also for the operators with the kernels $\psi\left(x_{1}\right)\left(\sigma_{1}^{*} E_{I, t}^{\bullet}\right)_{x_{1}, y a} \varphi\left(y_{1}\right)$, acting from smooth forms on $I$ into smooth forms on $[0, \pm 1]$ (where the Dirichlet boundary conditions are implied on $\partial I$ and $\sigma_{1}$ is the mirror symmetry with respect to $0 \in I$ ).

The $C^{2 k}$-norm of the kernel $\left(P_{N, t}^{\bullet(m)}-E_{N, t}^{\bullet}\right)$ on $N \times N$ is $O\left(t^{m-(n-1) / 2-k}\right)$ for $t \in(0, T]$, where $P_{N, t}^{\bullet(m)}$ and $E_{N, t}^{\bullet}$ are the parametrix of the type (2.124) and the fundamental solution for $\left(\partial_{t}+\Delta_{N}^{\bullet}\right)$ ([BGV], Theorem 2.30). The operators in $D R^{\bullet}(N)$ corresponding to $P_{N, t}^{\bullet(m)}$ are uniformly bounded for $t \in(0, T]$ with respect to a $C^{2 k_{-}}$ norm ([BGV], Lemma 2.49). So the operators $\exp \left(-t \Delta_{N}^{\bullet}\right)$ in $D R^{\bullet}(N)$ are uniformly bounded for $t \in(0, T]$ with respect to a $C^{2 k}$-norm. The convergence of the series on the right in $(2.137)$ with respect to $C^{2 k}$-norms $(k \leq[-n / 2]+m-1)$ for the kernels on $\bar{M}_{j_{1}} \times \bar{M}_{j_{2}}$ involves also a proof of the equality (2.137) (in the case of $m \geq-[-n / 2]+2)$. Indeed, we have ${ }^{42}$

$$
\left(\partial_{t}+\Delta_{\nu, x}^{\bullet}\right)\left(P_{l}^{\bullet(m)}\right)_{t, x, y}=\left(r_{l}^{\bullet(m)}+r_{l+1}^{\bullet(m)}\right)_{t, x, y},
$$

\footnotetext{
${ }^{41}$ Here $\sigma_{i}=t_{i+1}-t_{i}$ for $1 \leq i \leq k-1, \sigma_{0}=t_{1}, \sigma_{k}=1-t_{k}$. The volume of $\Delta_{k}$ with respect to the density $d t_{1} \ldots d t_{k}$ is equal to $1 / k$ !.

${ }^{42}$ The proof of (2.138) is analogous to the one given in [BGV], Lemma 2.22. It follows from the formula for $\partial_{t} \int_{0}^{t} f(x, t) d x$.
} 
where $(-1)^{m} P_{l}^{\bullet(m)}$ is the term with the number $l$ in the right side of $(2.137),(-1)^{m} r_{l}^{\bullet(m)}$ is the same term in which $P_{\sigma_{0} t}^{\bullet(m)}$ is replaced by $r_{\sigma_{0} t}^{\bullet(m)}, P_{0}^{\bullet(m)}:=P^{\bullet(m)}$. For any fixed $y$ and $t>0$ and for any $k \in \mathbb{Z}_{+} \cup 0$ we have $\left(\Delta_{\nu, x}^{k}\right)\left(P_{l}^{\bullet(m)}\right)_{t, x, y} \subset D\left(\Delta_{\nu, x}^{\bullet}\right)$. The series $P_{t}^{\bullet}=\sum_{l \geq 0}(-1)^{m} P_{l}^{\bullet(m)}$ for $m \geq[-n / 2]+2$ is the fundumental solution for $\left(\partial_{t}+\Delta_{\nu}^{\bullet}\right)$ since $\left(\partial_{t}+\Delta_{\nu, x}^{\bullet}\right) P_{t}^{\bullet}=0$ for $t>0$ and since the operator corresponding to $P_{t}^{\bullet(m)}$ tends in a weak sense to the identity operator in $\left(D R^{\bullet}(M)\right)_{2}$ as $t \rightarrow+0$ (i.e., $P_{t}^{\bullet(m)} \omega \rightarrow \omega$ as $t \rightarrow+0$ for $\omega \in\left(D R^{\bullet}(M)\right)_{2}$ ). The latter assertion holds for $P_{t, i n t}^{\bullet(m)}(1-\varphi) \omega$ and for $\mathcal{P}^{\bullet(m)} \varphi \omega$. Proposition 2.21 is proved.

Proof of Proposition 2.19. Proposition 2.18 involves the following conclusion. For any $\varepsilon>0$ there exist a $\delta>0$ and a neighborhood $U:=U\left(\nu_{0}, \varepsilon\right) \subset \mathbb{R}^{2} \backslash(0,0)$ of $\nu_{0}$ such that the estimate holds uniformly with respect to $\nu \in U$ and to $s,-1<s<1$ :

$$
\left|\int_{0}^{\delta} t^{s-1} d t \int_{M_{[-1 / 2,1 / 2]}} \sum_{k=1,2} \operatorname{tr}\left(* i_{k}^{*} e_{t}^{\bullet}(\nu)\right)\right|<\varepsilon / 20 .
$$

So it is enough to prove the existence of a neighborhood $U_{1}$ of $\nu_{0}$ such that for any $\nu \in U_{1}$ the following estimate holds uniformly with respect to $s$ for $-(1-b)<s<1$ $(b, 0<b<1$, is fixed):

$$
\int_{\delta}^{T} t^{s-1} d t\left|\int_{M_{[-1 / 2,1 / 2]}} \sum_{k=1,2}\left(\operatorname{tr}\left(* i_{k}^{*} e_{t}^{\bullet}(\nu)\right)-\operatorname{tr}\left(* i_{k}^{*} e_{t}^{\bullet}\left(\nu_{0}\right)\right)\right)\right|<\varepsilon / 10 .
$$

For $e(\nu)$ and $e\left(\nu_{0}\right)$ the equalities (2.121) hold. So the estimate (2.139) takes place for $\nu$ sufficiently close to $\nu_{0}$ since the convergence

$$
E_{t, x, y}^{\bullet}(\nu) \rightarrow E_{t, x, y}^{\bullet}\left(\nu_{0}\right)
$$

is uniform with respect to $t \in\left[\delta_{1}, T\right]$ (where $\delta_{1}>0$ is fixed), to $x \in M \backslash((-3 / 4,3 / 4) \times N)$, and to $y \in M_{[-1 / 2,1 / 2]}$. The convergence of the kernels

$$
d_{x} E_{t, x, y}^{\bullet}(\nu) \rightarrow d_{x} E_{t, x, y}^{\bullet}\left(\nu_{0}\right), \quad \delta_{x} E_{t, x, y}^{\bullet}(\nu) \rightarrow \delta_{x} E_{t, x, y}^{\bullet}\left(\nu_{0}\right) .
$$

is a uniform one for such $(t, x, y)$. All the double forms in (2.140) and (2.141) are uniformly bounded on the set of such $(t, x, y)$ and their norms at $(t, x, y)$ satisfy the upper estimate for $t \in\left(0, \delta_{1}\right.$ ] (obtained in Proposition 2.18 above) through $c_{m} t^{m}$ with an arbitrary $m \in \mathbb{Z}_{+}$and with $c_{m}$ independent of $\nu$. The uniform convergence of the kernels in (2.140) and (2.141) on the compact set of $(t, x, y)$ defined above follows from the continuity in $t, x, y$, and $\nu$ for $(x, y) \in \bar{M}_{j_{1}} \times \bar{M}_{j_{2}}$ of the corresponding double forms. (See Proposition 3.2, where it is proved that these double forms are $C^{\infty}$-smooth in $t, x, y$, and $\nu$ for $\operatorname{Re} t>0$ and $\nu \neq(0,0)$.) 
Proof of Proposition 2.20. If $\left(\alpha_{0}, \beta_{0}\right)=\nu_{0} \in U$ (i.e., if $\left.\alpha_{0} \cdot \beta_{0} \neq 0\right)$ then we can suppose that $\nu \in U$ in (2.120). In this case, the identity (2.100) holds for the difference

$$
\left(E^{\bullet}(\nu)-E^{\bullet}\left(\nu_{0}\right)\right)_{t, x_{1}, x_{2}} .
$$

Let $\nu_{0} \in \mathbb{R}^{2} \backslash(U \cup(0,0))$. For example, let $\nu_{0}:=\left(\alpha_{0}, 0\right), \alpha_{0} \neq 0$. Then the identities (2.98) and (2.99) claim in the cases of $\nu_{0}$ and of $\nu:=(\alpha, \beta)$ that (2.142) is equal to

$$
\begin{aligned}
&\left(\frac{\beta}{\alpha}\right) \lim _{\varepsilon \rightarrow+0} \int_{\varepsilon}^{t-\varepsilon} d \tau\left[-\left(r_{*, \partial M_{2}}\right.\right.\left.\delta E_{\tau, x_{1}, *}^{\bullet}(\nu), A_{*, \partial M_{1}} E_{t-\tau, *, x_{2}}^{\bullet}\left(\nu_{0}\right)\right)_{N}+ \\
&+\left(A_{*, \partial M_{1}} E_{\tau, x_{1}, *}^{\bullet}(\nu), r_{*, \partial M_{2}} \delta_{*} E_{t-\tau, *, x_{2}}^{\bullet}\left(\nu_{0}\right)\right)_{N}+\left(A_{*, \partial M_{1}} d_{*} E_{\tau, x_{1}, *}^{\bullet}(\nu), r_{*, \partial M_{2}} E_{t-\tau, *, x_{2}}^{\bullet}\left(\nu_{0}\right)\right)_{N}- \\
&\left.\quad-\left(r_{*, \partial M_{2}} E_{\tau, x_{1}, *}^{\bullet}(\nu), A_{*, \partial M_{1}} d_{*} E_{t-\tau, *, x_{2}}^{\bullet}\left(\nu_{0}\right)\right)_{N}\right] . \quad(2.143)
\end{aligned}
$$

The factor $k_{\nu}^{-1}=\beta / \alpha$ in (2.143) tends to zero as $\nu$ tends to $\nu_{0}$. The factors $\left(1-k_{\nu} / k_{\nu_{0}}\right)$ and $\left(1-k_{\nu_{0}} / k_{\nu}\right)$ in $\left.(2.100)\right)$ also tend to zero as $\nu \rightarrow \nu_{0}$ in the case $\nu_{0}, \nu \in U$. The estimate (2.120) follows from (2.143) and (2.100). Indeed, there are the uniform with respect to $\nu$ upper estimates (analogous to (2.116)) for the kernels (2.122), where $x, y \in M \backslash M_{[-1 / 2,1 / 2]}$ and $z \in N=0 \times N \subset \partial M_{j}$. These estimates follow from (2.32) and their proofs are completely analogous to the proof of (2.116). The main step in these proofs is using of the parametrix $P^{(m) 43}$ and of the estimates (2.127), (2.128), and (2.132) for $t \in(0, T]$.

2.8. Dependence on the phase of a cut of the spectral plane. The analytic torsions as functions of the phase of a cut. Gluing formula for the analytic torsions. The scalar analytic torsion (2.11) depends not only on $\left(M, g_{M}, Z, \nu\right)$ but also on the phase $\theta$ of a cut on the spectral plane $\mathbb{C} \ni \lambda$. A zeta-function $\zeta_{\nu, \bullet}(s ; \theta)$ is defined for $\operatorname{Re} s>n / 2(n:=\operatorname{dim} M)$ as the sum of absolutely convergent series $\sum m\left(\lambda_{j}\right) \lambda_{j, \theta}^{-s}$, where the sum is over nonzero $\lambda_{j} \in \operatorname{Spec}\left(\Delta_{\nu}^{\bullet}\right)$ and $m\left(\lambda_{j}\right)$ are the multiplicities of $\lambda_{j}$. The function $\lambda_{j, \theta}^{-s}:=\exp \left(-s \log _{(\theta)} \lambda_{j}\right), \theta>\operatorname{Im}\left(\log _{(\theta)} \lambda_{j}\right)>\theta-2 \pi$, is defined for $\theta \notin \arg \lambda_{j}+2 \pi \mathbb{Z}$. (For positive self-adjoint operators this condition means that $\theta \notin 2 \pi \mathbb{Z}$.) All the results for the analytic torsion norm are obtained above in the case of $0<\theta<2 \pi$ (for instance, for $\theta=\pi$ ).

The zeta-function $\zeta_{\nu, \bullet}(s ; \theta)$ does not depend on $\theta \notin 2 \pi \mathbb{Z}$, if $[\theta / 2 \pi]$ does not change. However we have

$$
\zeta_{\nu, \bullet}(s ; \theta+2 \pi)=\exp (-2 \pi i s) \zeta_{\nu, \bullet}(s ; \theta) \quad \text { for } \operatorname{Re} s>n / 2
$$

\footnotetext{
${ }^{43}$ The parametrix $P^{(m), \bullet}(\nu)$ for $E^{\bullet}(\nu)$ can be chosen such that $P_{t, x, z}^{(m), \bullet}(\nu)=0$ for $x \notin[-1 / 3,1 / 3] \times$ $N \hookrightarrow M$ and $z \in[-1 / 6,1 / 6] \times N \hookrightarrow M$.
} 
Since $\zeta_{\nu, \bullet}(s ; \pi)$ can be meromorphically continued to the complex plane $\mathbb{C} \ni s$ (Theorem 3.1 below), we see that $\zeta_{\nu, \bullet}(s ; \theta)$ for $\theta \notin 2 \pi \mathbb{Z}$ also can be meromorphically continued to $\mathbb{C}$ with at most simple poles at $s_{j}:=(n-j) / 2$. The continuation of $\zeta_{\nu, \bullet}(s ; \theta)$ is regular at $s_{j}$ for $\left(-s_{j}\right) \in \mathbb{Z}_{+} \cup 0$. So the equality $(2.144)$ holds for all $\theta \notin 2 \pi \mathbb{Z}, s \in \mathbb{C}$. Hence for such $\theta$ we have

$$
\zeta_{\nu, \bullet}(s ; \theta)=\zeta_{\nu, \bullet}(s) \exp (-2 \pi i s m),
$$

where $\zeta_{\nu, \bullet}(s):=\zeta_{\nu, \bullet}(s ; \pi)$ and $m:=[\theta / 2 \pi], \theta \notin 2 \pi \mathbb{Z}$. From now on this $\zeta$-function will be denoted as $\zeta_{\nu, \bullet}(s ; m)$ with $m=[\theta / 2 \pi]$. The value $\zeta_{\nu, \bullet}(0, m)$ is independent of $m$ (according to $(2.145)$ ).

The dependence of the scalar analytic torsion (2.11) on $m$ is given by

$$
T\left(M_{\nu}, Z ; m\right)=T\left(M_{\nu}, Z\right) \exp \left(-2 \pi i m F\left(M_{\nu}, Z\right),\right)
$$

where $^{44} F\left(M_{\nu}, Z\right):=\sum(-1)^{j} j \zeta_{\nu, \bullet}(0)(\bmod \mathbb{Z})$ and $T\left(M_{\nu}, Z\right):=T\left(M_{\nu}, Z ; 0\right)$, i.e., $T\left(M_{\nu}, Z\right)$ corresponds to $\theta=\pi$ and it is the scalar analytic torsion defined by $(2.11)$. Here $Z$ is the union of the connected components of $\partial M$ where the Dirichlet boundary conditions are given. The Laplacian $\Delta_{\nu}^{\bullet}$ is defined on $\omega \in D R^{\bullet}\left(M_{\nu}, Z\right)$ with the $\nu$ transmission boundary conditions $(1.27)$ on $N$, with the Dirichlet and the Neumann boundary conditions on $Z$ and on $\partial M \backslash Z$. The equality (2.146) is obtained by using of

$$
\left.\partial_{s} \zeta_{\nu, \bullet}(s ; m)\right|_{s=0}=-2 \pi i m \zeta_{\nu, \bullet}(0)+\left.\partial_{s} \zeta_{\nu, \bullet}(s)\right|_{s=0} .
$$

The number $F(M, Z)$ is defined also in the case of a manifold $M$ without an interior boundary $N$. In this case, $\zeta_{\nu, \bullet}(0)$ in the definition of $F(M, Z)$ is replaced by $\zeta_{\bullet}(0)$ for the Laplacians on $D R^{\bullet}(M, Z)$. The dependence of $T(M, Z ; m)$ on $m$ is given by (2.146) with $F\left(M_{\nu}, Z\right)$ replaced by $F(M, Z)$. In particular, $F(M)$ is defined for a closed $M$ and also in the case $\partial M \neq \emptyset, Z=\emptyset$. Let $M$ be obtained by gluing two pieces $M_{1}$ and $M_{2}$ along the common component $N$ of their boundaries, $M=M_{1} \cup_{N} M_{2}$, where $N \subset M$ is closed and of codimension one. Then $F(M, Z)=F\left(M_{1,1}, Z\right)$, according to Proposition 1.1.

The class of $F\left(M_{\nu}, Z\right)$ in $\mathbb{C} / \mathbb{Z}$ is the same as the class $(\bmod \mathbb{Z})$ of the number $F_{1}\left(M_{\nu}, Z\right) \in \mathbb{C}$, where

$$
F_{1}\left(M_{\nu}, Z\right):=\sum(-1)^{j} j\left(\zeta_{\nu, j}(0)+\operatorname{dim} \operatorname{Ker}\left(\Delta_{\nu, j}\right)\right) .
$$

The Laplacian $\Delta_{\nu, j}\left(M_{\nu}, Z\right)$ with its domain $\operatorname{Dom}\left(\Delta_{\nu, j}\right) \subset\left(D R^{j}(M)\right)_{2}$ is selfadjoint according to Theorem 3.1. For $\operatorname{Re} s>2^{-1} \operatorname{dim} M$ the zeta-function $\zeta_{\nu, j}(s)$ is defined by the absolutely convergent series $\sum m\left(\lambda_{k}\right) \exp \left(-s \log \lambda_{k}\right)([\theta / 2 \pi]=0$, $\theta \neq 0)$, where the sum is over $\lambda_{k} \in \operatorname{Spec}\left(\Delta_{\nu, j}\right), \lambda_{k} \neq 0$, and with the branch of $\operatorname{logarithm}-\pi<\operatorname{Im} \log \lambda<\pi$. Because $\log \lambda_{k} \in \mathbb{R}$ for $\lambda_{k}>0$, the function $\zeta_{\nu, j}(s)$ is

\footnotetext{
${ }^{44}$ By the definition, $F\left(M_{\nu}, Z\right) \in \mathbb{C} / \mathbb{Z}$ but it can be also defined as $\sum(-1)^{j} j \zeta_{\nu, j}(0) \in \mathbb{C}$.
} 
real for real $s$. Hence $F_{1}\left(M_{\nu}, Z\right) \in \mathbb{R}$. It is supposed from now on that a metric $g_{M}$ on $M=M_{1} \cup_{N} M_{2}$ is a direct product metric near $N$ and near $\partial M$.

Proposition 2.22. 1. For a closed manifold $M$ the number $F_{1}(M)$ is an integer.

2. Let $M=M_{1} \cup_{N} M_{2}$ be obtained by gluing two its pieces $M_{1}$ and $M_{2}$ along the common component $N$ of their boundaries. Let the $\nu$-transmission boundary conditions (1.27) be given on $N$, the Dirichlet boundary conditions be given on a union $Z$ of some connected components of $\partial M$ and the Neumann boundary conditions be given on $\partial M \backslash Z$. Let $L$ be a closed Riemannian manifold. Then the following holds: ${ }^{45}$

$$
F_{1}\left(M_{\nu} \times L, Z \times L\right)=\chi(L) F_{1}\left(M_{\nu}, Z\right)+F_{1}(L) \chi\left(M_{\nu}, Z\right) .
$$

3. Let $K \subset \partial M \backslash Z$ be a union of some connected components of $\partial M$. Then the following holds under the conditions on $M$ above:

$$
F_{1}\left(M_{\nu}, Z\right)=F_{1}\left(M_{\nu}, Z \cup K\right)+F_{1}(K)+2^{-1} \chi(K) .
$$

4. Under the conditions on $M$ above, the number $F_{1}\left(M_{\nu}, Z\right)$ obeys a gluing property analogous to the gluing property (2.1) for the analytic torsion norms. Namely the following holds:

$$
F_{1}\left(M_{\nu}, Z\right)=F_{1}\left(M_{1}, Z_{1} \cup N\right)+F_{1}\left(M_{2}, Z_{2} \cup N\right)+F_{1}(N)+2^{-1} \chi(N),
$$

where $Z_{k}:=Z \cap \partial \bar{M}_{k}$.

Corollary 2.9. 1. For a closed $M$ the scalar analytic torsion $T(M,[\theta / 2 \pi])$ is independent of $\theta \notin 2 \pi \mathbb{Z}$.

2. Under the conditions of (2.147), (2.148), and (2.149), the following holds in $\mathbb{R} / \mathbb{Z}:$

$$
\begin{gathered}
F\left(M_{\nu} \times L, Z \times L\right)=\chi(L) F\left(M_{\nu}, Z\right) . \\
F\left(M_{\nu}, Z\right)=F\left(M_{\nu}, Z \cup K\right)+2^{-1} \chi(K) . \\
F\left(M_{\nu}, Z\right)=F\left(M_{1}, Z_{1} \cup N\right)+F\left(M_{2}, Z_{2} \cup N\right)+2^{-1} \chi(N) .
\end{gathered}
$$

Example 2.1. The number $F_{1}\left(S^{1}\right)$ is equal to $-f_{0 ; 1}=-f_{0 ; 0} \cdot{ }^{46}$ The latter one is equal to zero because the asymptotic expansion for $\operatorname{Tr} \exp \left(-t \Delta_{0}\left(S^{1}\right)\right)$ as to $t \rightarrow+0$ (where $\Delta_{0}$ is the Laplacian on functions) is $f_{-1 ; 0} t^{-1 / 2}+f_{1 ; 0} t^{1 / 2}+f_{3 ; 0} t^{3 / 2}+\ldots$

\footnotetext{
${ }^{45}$ The Euler characteristic $\chi\left(M_{\nu}, Z\right):=\sum(-1)^{i} \operatorname{dim} H^{i}\left(M_{\nu}, Z\right)$ is equal to the Euler characteristic of the complex $\left(C^{\bullet}\left(X_{\nu}, X \cap Z\right), d_{c}\right)$ (as it follows from Proposition 2.3). Hence it is equal to $\chi(M, Z)$ and is independent of $\nu \in \mathbb{R}^{2} \backslash(0,0)$.

${ }^{46}$ The coefficients $f_{k, j}:=f_{k, j}(M, Z)$ are the coefficients in the asymptotic expansion $\sum f_{k, j} t^{k / 2}$ $(k \geq-n)$ for $\operatorname{Tr} \exp \left(-t \Delta_{j}(M, Z)\right)$ as $t \rightarrow+0$ for the Laplacian on $D R^{j}(M, Z)(n:=\operatorname{dim} M)$.
} 
Example 2.2. The number $F_{1}(I, \partial I)$ is equal to $-f_{0 ; 1}(I, \partial I)=-f_{0 ; 0}(I)$. Since $S^{1}$ has a mirror symmetry relative to its diameter, we have, taking into account (2.54) and (2.118),

$$
f_{0,0}\left(S^{1}\right)=f_{0 ; 0}(I)+f_{0 ; 0}(I, \partial I)=0 .
$$

Since $f_{0 ; 0}(I, \partial I)-f_{0 ; 1}(I, \partial I)=\chi(I, \partial I)=1$ and (2.150) holds, we see that

$$
F_{1}(I, \partial I)=-f_{0 ; 1}(I, \partial I)=-f_{0 ; 0}(I)=f_{0 ; 0}(I, \partial I)=-2^{-1} .
$$

Remark 2.10. The equality (2.151) means that the analytic torsion $T(I, \partial I ;[\theta / 2 \pi])$ is multiplied by the factor $\exp \left(-2 \pi i \cdot\left(-2^{-1}\right)\right)=-1$, if $\theta$ is replaced by $\theta+2 \pi$ $(\theta \notin 2 \pi \mathbb{Z})$.

It is necessary to note the following. The scalar analytic torsion $T(I, \partial I):=$ $T(I, \partial I ; \pi)$ is the factor in the analytic torsion norm. But the latter one is the square of the norm on the determinant line $\operatorname{det} H^{1}(I, \partial I)$. So the factor, corresponding to the norm itself, is multiplied in the case of $(M, Z)=(I, \partial I)$ by the factor

$$
\exp \left(\left.\pi i \zeta_{1}(I, \partial I)\right|_{s=0}\right)=\exp (-\pi i / 2)=-i,
$$

if $\theta$ is replaced by $\theta+2 \pi, \theta \notin 2 \pi \mathbb{Z}$. Indeed, we have

$$
-2^{-1}=F_{1}(I, \partial I)=-\left(\operatorname{dim} H^{1}(I, \partial I)+\left.\zeta_{1}(I, \partial I)\right|_{s=0}\right),
$$

and so $\left.\zeta_{1}(I, \partial I)\right|_{s=0}=-2^{-1}=-F(I, \partial I)$. For $M=S^{1}$ it holds that $-F\left(S^{1}\right)=$ $\left.\zeta_{1}\left(S^{1}\right)\right|_{s=0}=-1$, and so $\exp \left(-\pi i F\left(S^{1}\right)\right)=-1$.

It follows from Proposition 2.23 below that $F\left(M_{\nu}, Z\right)$ and $F(M, Z)$ have a form $(1 / 2)+\mathbb{Z}$, if the numbers $n:=\operatorname{dim} M$ and $\chi(M, Z)$ are odd. So in this case the factors $\exp \left(-\pi i F\left(M_{\nu}, Z\right)\right)$ and $\exp (-\pi i F(M, Z))$ are equal to $\{ \pm i\}$.

Proof of Proposition 2.22. 1. Theorem 3.2 1. claims that the number $\zeta_{\nu, j}(0)+$ $\operatorname{dim} \operatorname{Ker}\left(\Delta_{\nu, j}\right)$ is equal to the constant term $f_{0 ; j}\left(M_{\nu}, Z\right)$ in the asymptotic expansion (2.87) for $\operatorname{Tr} \exp \left(-t \Delta_{\nu, j}\right)$ as $t \rightarrow+0$. So according to Theorem 3.21 . the number $F_{1}\left(M_{\nu}, Z\right)$ is equal to the sum of the integrals over $M_{1}, M_{2}, N$, and $\partial M$ of the locally defined densities. Then we have

$$
F_{1}\left(M_{\nu}, Z\right)=\sum(-1)^{j} j f_{0 ; j}\left(M_{\nu}, Z\right) .
$$

If $\left(M, g_{M}\right)$ is a closed Riemannian manifold then $f_{0 ; j}(M)=f_{0 ; n-j}(M)$. Hence taking into account (2.152) and (2.57), we get (for even $n:=\operatorname{dim} M$ )

$$
F_{1}(M)=\sum(-1)^{j} j f_{0 ; j}(M)=(n / 2) \sum(-1)^{j} f_{0 ; j}(M)=(n / 2) \chi(M) .
$$

Let $n$ be odd. Then $f_{0 ; j}(M)$ is equal to zero since the asymptotic expansion for $\operatorname{tr} \exp \left(-t \Delta_{j}\left(M, g_{M}\right)\right)($ as $t \rightarrow+0)$ is $t^{-n / 2} \sum t^{l} f_{2 l-n ; j}\left(M, g_{M}\right)$, where the sum is over 
$l \in \mathbb{Z}_{+} \cup 0\left([\mathrm{Gr}]\right.$, Theorem 1.6.1; $[\mathrm{BGV}]$, Theorem 2.30). Hence $F_{1}(M)=0=$ $(n / 2) \chi(M)$ for an odd $n$ also.

This number $(n / 2) \chi(M)$ is an integer for any closed $M$. (The assertion that $F_{1}(M)$ is an integer follows also from the equality which holds for any closed even-dimensional Riemannian $\left(M, g_{M}\right)$ :

$$
\sum j(-1)^{j} \zeta_{j}(M, s)=\sum j(-1)^{j} \zeta_{n-j}(M, s)=(n / 2) \sum(-1)^{j} \zeta_{j}(M, s)=0,
$$

because $\zeta_{j}(M, s)=\zeta_{n-j}(M, s)$.)

2. Let $\lambda \in \operatorname{Spec}\left(\Delta_{\nu, j}(M, Z)\right), \mu \in \operatorname{Spec}\left(\Delta_{i}(L)\right)$ and let $m_{\lambda}\left(j ; M_{\nu}, Z\right), m_{\mu}(i ; L)$ be their multiplicities. If $\lambda \neq 0$ and $\mu \neq 0$ then we have

$$
\sum(-1)^{j+i}(j+i) m_{\lambda}\left(j ; M_{\nu}, Z\right) m_{\mu}(i ; L)=0,
$$

since the subcomplexes $\left(V_{\lambda}^{\bullet}\left(M_{\nu}, Z\right), d\right) \hookrightarrow\left(D R^{\bullet}\left(M_{\nu}, Z\right), d\right)$ and $\left(V_{\mu}^{\bullet}(L), d\right) \hookrightarrow$ $\left(D R^{\bullet}(L), d\right)$, corresponding to the $\lambda$-eigenforms for $\Delta_{\nu, \bullet}(M, Z)$ and to the $\mu$-eigenforms for $\Delta_{\mathbf{\bullet}}(L)$, are acyclic. If $\lambda \neq 0$ but $\mu=0$ then the right side of (2.154) is equal to

$$
\left(\sum(-1)^{j} j m_{\lambda}\left(j ; M_{\nu}, Z\right)\right)\left(\sum(-1)^{i} \operatorname{dim} \operatorname{Ker} \Delta_{i}(L)\right)==\chi(L) \sum(-1)^{j} j m_{\lambda}\left(j ; M_{\nu}, Z\right) .
$$

So under the conditions of 2 , by using (1.37), we have

$$
\begin{aligned}
& \left.\sum(-1)^{j} j \zeta_{\nu, j}\left(M_{\nu} \times L, Z \times L\right)\right|_{s=0}= \\
& \quad=\left.\chi(L) \sum(-1)^{j} j \zeta_{\nu, j}\left(M_{\nu}, Z\right)\right|_{s=0}+\left.\chi\left(M_{\nu}, Z\right) \sum(-1)^{j} j \zeta_{j}(L)\right|_{s=0}, \quad(2.155) \\
& \sum(-1)^{j} j \operatorname{dim} \operatorname{Ker}\left(\Delta_{\nu, j}\left(M_{\nu} \times L, Z \times L\right)\right)= \\
& =\chi(L) \sum(-1)^{j} j \operatorname{dim} \operatorname{Ker}\left(\Delta_{\nu, j}\left(M_{\nu}, Z\right)\right)+\chi\left(M_{\nu}, Z\right) \sum(-1)^{j} j \operatorname{dim} \operatorname{Ker}\left(\Delta_{j}(L)\right) .
\end{aligned}
$$

The equality (2.147) follows now from (2.155) and (2.156).

3. The numbers $F_{1}\left(M_{\nu}, Z\right)$ and $F_{1}\left(M_{\nu}, Z \cup K\right)$ are the sums of the integrals over $M_{1}, M_{2}, N$, and $\partial M$ of the locally defined densities (as it follows from (2.152) and from Theorem 3.2). The densities, corresponding to the pairs $\left(M_{\nu}, Z\right)$ and $\left(M_{\nu}, Z \cup\right.$ $K)$, differ only on $K$. So the difference $F_{1}\left(M_{\nu}, Z\right)-F_{1}\left(M_{\nu}, Z \cup K\right)$ depends only on $K$ and on $g_{M}$ near $K$. Thus taking into account that $g_{M}$ is a direct product metric near $K$, we get

$$
2\left(F_{1}\left(M_{\nu}, Z\right)-F_{1}\left(M_{\nu}, Z \cup K\right)\right)=F_{1}(K \times I)-F_{1}(K \times(I, \partial I))
$$

for any fixed metric on $K$ in all the terms of this equality. 
According to (2.147) we have

$$
\begin{aligned}
F_{1}(K \times I) & =F_{1}(K) \chi(I)+F_{1}(I) \chi(K), \\
F_{1}(K \times(I, \partial I)) & =F_{1}(K) \chi(I, \partial I)+F_{1}(I, \partial I) \chi(K), \\
F_{1}(K \times I)-F_{1}(K \times(I, \partial I)) & =2 F_{1}(K)+\chi(K)\left(F_{1}(I)-F_{1}(I, \partial I)\right) .
\end{aligned}
$$

Since $\zeta_{1}(s ; I)=\zeta_{1}(s ; I, \partial I)$ (on the same $I$ ) we have

$$
F_{1}(I)-F_{1}(I, \partial I)=-\left.\zeta_{1}(I)\right|_{s=0}+\left.\zeta_{1}(I, \partial I)\right|_{s=0}+\operatorname{dim} H^{1}(I, \partial I)=1 .
$$

By (2.157) and (2.158) we get

$$
F_{1}\left(M_{\nu}, Z\right)=F_{1}\left(M_{\nu}, Z \cup K\right)+F_{1}(K)+2^{-1} \chi(K) .
$$

4. The number $F_{1}\left(M_{\nu}, Z\right)$ is the sum of the integrals over $M_{1}, M_{2}, N$, and over $\partial M$ of the locally defined densities. (It is a consequence of Theorem 3.2). So the densities on $M_{j}, N$, and on $\partial M \cap \bar{M}_{j}$ are the same as for the number $F_{1}\left(M_{j, \nu}^{(2)}, Z_{j}^{(2)}\right)$, where $M_{j}^{(2)}:=M_{j} \cup_{N} M_{j}, Z_{j}^{(2)}:=Z_{j} \cup Z_{j}$, and $g_{M_{j}^{(2)}}$ are mirror symmetric with respect to $N$ (the $\nu$-transmission boundary conditions are given on $N \hookrightarrow M_{j}^{(2)}$ ) and $\left.g_{M_{j}^{(2)}}\right|_{M_{j}}=\left.g_{M}\right|_{M_{j}}$. Thus we have

$$
2 F_{1}\left(M_{\nu}, Z\right)=\sum_{j=1,2} F_{1}\left(M_{j, \nu}^{(2)}, Z_{j}^{(2)}\right)
$$

Since pairs $\left(M_{j}^{(2)}, Z_{j}^{(2)}\right)$ are mirror symmetric with respect to $N$, it follows from $(2.54)$ and (2.55) that

$$
\begin{gathered}
F_{1}\left(M_{j, \nu}^{(2)}, Z_{j}^{(2)}\right)=F_{1}\left(M_{j}^{(2)}, Z_{j}^{(2)}\right)=F_{1}\left(M_{j}, Z_{j}\right)+F_{1}\left(M_{j}, Z_{j} \cup N\right), \\
F_{1}\left(M_{\nu}, Z\right)=2^{-1} \sum_{j=1,2}\left(F_{1}\left(M_{j}, Z_{j}\right)+F_{1}\left(M_{j}, Z_{j} \cup N\right)\right) .
\end{gathered}
$$

The equality (2.149) follows from (2.148) with $M=M_{j}, Z=Z_{j}, K=N$, and $\nu=(1,1)$ and from $(2.160)$, because $F_{1}\left(M_{j}, Z_{j}\right)=F_{1}\left(M_{j}, Z_{j} \cup N\right)+F_{1}(N)+2^{-1} \chi(N)$. Thus Proposition 2.22 is proved.

The analytic torsion $T_{0}\left(M_{\nu}, Z ; m\right)$ (where $Z \subset \partial M$ is a union of some connected components of $\partial M, m:=[\theta / 2 \pi], \theta \notin 2 \pi \mathbb{Z})$ is defined as the product of the norm $\|\cdot\|_{\operatorname{det} H \bullet\left(M_{\nu}, Z\right)}^{2}$ (given by the natural norm on harmonic forms for $\Delta_{\nu}^{\bullet}\left(M_{\nu}, Z\right)$ ) and of the scalar analytic torsion $T\left(M_{\nu}, Z ; m\right)$ :

$$
T_{0}\left(M_{\nu}, Z ; m\right):=\|\cdot\|_{\operatorname{det} H \bullet\left(M_{\nu}, Z\right)}^{2} T\left(M_{\nu}, Z ; m\right) .
$$

The analytic torsion $T_{0}(M, Z ; m)$ is the norm $\|\cdot\|_{\operatorname{det} H \bullet(M, Z)}^{2} T(M, Z ; m)$, where the norm on the determinant line is given by the harmonic forms for $\Delta_{\bullet}(M, Z)$. 
(If $N$ is the interior boundary and if $g_{M}$ is a direct product metric near $N$ then $T_{0}(M, Z ; m)=T_{0}\left(M_{1,1}, Z ; m\right)$ according to Proposition 1.1.)

Theorem 2.1. 1. Let $M$ be obtained by gluing two pieces along $N, M=M_{1} \cup_{N} M_{2}$, where $N$ is a closed of codimension one submanifold in $M$ with a trivial normal bundle $\left.T M\right|_{N} / T N$ and $g_{M}$ is a direct product metric near $N$ and near $\partial M$. Then for $\nu \in \mathbb{R}^{2} \backslash(0,0)$ the following gluing formula holds:

$$
\begin{aligned}
\varphi_{\nu}^{a n} T_{0} & \left(M_{\nu}, Z ; m\right):= \\
& =(-1)^{m \chi(N)} T_{0}\left(M_{1}, Z_{1} \cup N ; m\right) \otimes T_{0}\left(M_{2}, Z_{2} \cup N ; m\right) \otimes T_{0}(N ; m),
\end{aligned}
$$

where the identification $\varphi_{\nu}^{a n}$ of the determinants lines is defined by the short exact sequence (1.14) of the de Rham complexes and by Lemma 1.1, $Z_{k}:=Z \cap \partial \bar{M}_{k}$. The factor $T_{0}(N ; m):=T_{0}(N)$ is independent of $m$ (according to Proposition 2.221 ).

2. Let $K \subset \partial M \backslash Z$ be a union of some connected components of $\partial M$. Then the formula holds for gluing $K$ and $\left(M_{\nu}, Z \cup K\right)$ :

$$
\varphi_{a n} T_{0}\left(M_{\nu}, Z ; m\right)=(-1)^{m \chi(K)} T_{0}\left(M_{\nu}, Z \cup K ; m\right) \otimes T_{0}(K ; m) .
$$

Here the identification $\varphi_{\text {an }}$ is defined by the short exact sequence (analogous to (1.20)):

$$
0 \rightarrow D R^{\bullet}\left(M_{\nu}, Z \cup K\right) \rightarrow D R^{\bullet}\left(M_{\nu}, Z\right) \rightarrow D R^{\bullet}(K) \rightarrow 0
$$

(the left arrow in (2.164) is the natural inclusion and the right arrow is the geometrical restriction) and by Lemma 1.1. The factor $T_{0}(K ; m):=T_{0}(K)$ is independent of $m$. The analogous formula holds for gluing $K$ and $(M, Z \cup K)$ :

$$
\varphi_{a n} T_{0}(M, Z ; m)=(-1)^{m \chi(K)} T_{0}(M, Z \cup K ; m) \otimes T_{0}(K),
$$

where $\varphi_{a n}$ is defined by the short exact sequence (2.164) with $M_{\nu}$ replaced by $M$.

Proof. 1. For $T_{0}\left(M_{\nu}, Z\right):=T_{0}\left(M_{\nu}, Z ; 0\right)$ the following gluing formula holds (according to (1.12) and to Lemma 1.2):

$$
\varphi_{\nu}^{a n} T_{0}\left(M_{\nu}, Z\right)=T_{0}\left(M_{1}, Z_{1} \cup N\right) \otimes T_{0}\left(M_{2}, Z_{2} \cup N\right) \otimes T_{0}(N) .
$$

By the definition of $F\left(M_{\nu}, Z\right)$ we have

$$
T_{0}\left(M_{\nu}, Z ; m+1\right)=\exp \left(-2 \pi i F\left(M_{\nu}, Z\right)\right) T_{0}\left(M_{\nu}, Z ; m\right) .
$$

(Analogous equalities are true for $T_{0}\left(M_{j}, Z_{j} \cup N ; m\right)$. The differences $F_{1}\left(M_{\nu}, Z\right)-$ $F\left(M_{\nu}, Z\right), F_{1}\left(M_{j}, Z_{j} \cup N\right)-F\left(M_{j}, Z_{j} \cup N\right)$, and $F_{1}(N)-F(N)$ are integers and $F_{1}(N)$ is an integer (according to Proposition 2.221 ). Hence (2.162) is a consequence of (2.149) and (2.166).

2. The gluing formula holds for $T_{0}(M, Z):=T_{0}(M, Z ; 0)$ according to (1.18) (Theorem 1.2): $\varphi_{a n} T_{0}(M, Z)=T_{0}(M, Z \cup K) \otimes T_{0}(K)$. So the gluing formula $(2.165)$ follows from $(2.148)$ since $T_{0}(M, Z ; m+1)=\exp (-2 \pi i F(M, Z)) T_{0}(M, Z ; m)$ and since the difference $F_{1}(M, Z)-F(M, Z)$ is an integer. 
Let $N \subset M$ be a disjoint union $N_{1} \cup N_{2}$ of two closed codimension one submanifolds of $M$ with trivial normal bundles and let the $\nu_{j}$-transmission interior boundary conditions be given on $N_{j}$. Let $M=M_{1} \cup_{N_{1}} M_{2}$ and let $N_{2} \subset M_{1}$. Under these conditions, the equality (1.12) and the assertion of Lemma 1.2 are also true. Their proofs are similar to the given above. The resulting formula is

$$
\varphi_{\nu_{1}}^{a n} T_{0}\left(M_{\nu_{1}, \nu_{2}}, Z\right)=T_{0}\left(M_{1, \nu_{2}}, Z_{1} \cup N_{1}\right) \otimes T_{0}\left(M_{2}, Z_{2} \cup N_{1}\right) \otimes T_{0}\left(N_{1}\right) .
$$

(Here $Z \subset \partial M$ is a union of some connected components of $\partial M, Z_{k}=Z \cap \partial \bar{M}_{k}$ and $g_{M}$ is a direct product metric near $N_{j}$ and $\partial M$.) As a consequence of (2.167) (obtained by the same method as Theorem 1.2 is obtained from (1.16) and (1.17)) we get the following equality

$$
\varphi_{a n} T_{0}\left(M_{1, \nu_{2}}, Z_{1}\right)=T_{0}\left(M_{1, \nu_{2}}, Z_{1} \cup N_{1}\right) T_{0}\left(N_{1}\right) .
$$

The equality (2.163) follows from (2.168) (where $M_{1, \nu_{2}}, Z_{1}, N_{1}$ are replaced by $M_{\nu}$, $Z, K)$ and from $(2.148)$ since $T_{0}\left(M_{\nu}, Z ; m+1\right)=\exp \left(-2 \pi i F\left(M_{\nu}, Z\right)\right) T_{0}\left(M_{\nu}, Z ; m\right)$. Theorem 2.1 is proved.

Proposition 2.23. Let $M=M_{1} \cup_{N} M_{2}$ be obtained by gluing along $N$ and let the $\nu$-transmission boundary conditions $\left(\nu \in \mathbb{R}^{2} \backslash(0,0)\right)$ be given on $N$. Set $n:=\operatorname{dim} M$. Then the number $F_{1}\left(M_{\nu}, Z\right)$ for the scalar analytic torsion $T\left(M_{\nu}, Z ; m\right)$ is expressed by

$$
F_{1}\left(M_{\nu}, Z\right)=2^{-1} n \chi\left(M_{\nu}, Z\right)=2^{-1} n \chi(M, Z) .
$$

The number $F\left(M_{\nu}, Z\right):=\sum(-1)^{j} j \zeta_{\nu, j}(0)$ is as follows:

$$
F\left(M_{\nu}, Z\right)=\sum(-1)^{j}\left(-j+2^{-1} n\right) \operatorname{dim} H^{j}\left(M_{\nu}, Z\right) .
$$

Proof. 1. Proposition 2.3 claims that $\chi\left(M_{\nu}, Z\right)$ (i.e., the Euler characteristic $\left.\sum(-1)^{j} \operatorname{dim} H^{j}\left(D R\left(M_{\nu}, Z\right)\right)\right)$ is equal to the Euler characteristic for the finitedimensional complex $\left(C^{\bullet}\left(X_{\nu}, Z \cap X\right), d_{c}\right)$. Note that $\operatorname{dim} C^{j}(X, Z \cap X)$ is equal to $\operatorname{dim} C^{j}\left(X_{\nu}, Z \cap X\right)$. Hence $\chi\left(M_{\nu}, Z\right)=\sum(-1)^{j} \operatorname{dim} C^{j}(X, Z \cap X)$. This sum is equal to $\chi(M, Z)$ by the de Rham theorem ([RS], Proposition 4.2). Thus $\chi\left(M_{\nu}, Z\right)=$ $\chi(M, Z)$.

2. According to (2.149), the gluing formula holds:

$$
F_{1}\left(M_{\nu}, Z\right)=F_{1}\left(M_{1}, Z_{1} \cup N\right)+F_{1}\left(M_{2}, Z_{2} \cup N\right)+F_{1}(N)+2^{-1} \chi(N) .
$$

For the Euler characteristics the analogous formula holds:

$$
\chi\left(M_{\nu}, Z\right)=\chi(M, Z)=\chi\left(M_{1}, Z_{1} \cup N\right)+\chi\left(M_{2}, Z_{2} \cup N\right)+\chi(N) .
$$


The number $F_{1}(N)$ for a closed manifold $N$ is equal to $\chi(N)(\operatorname{dim} N) / 2$ by $(2.153)$. So $F_{1}(N)+2^{-1} \chi(N)=n \chi(N) / 2$. Let $\left(M, g_{M}\right)$ be a closed Riemannian manifold, mirror symmetric with respect to $N=\partial M_{1}, M=M_{1} \cup_{N} M_{1}$. Let $g_{M}$ be a direct product metric near $N$. Then the equality $F_{1}\left(M_{1}, N\right)=2^{-1} n \chi\left(M_{1}, N\right)$ follows from (2.153), which claims that $F_{1}(M)=2^{-1} n \chi(M)$, and from (2.170). So (2.169) holds for pairs $(M, Z)$, where $Z=\partial M$. For any union $Z$ of some connected components of $\partial M$ the equality (2.169) follows from (2.148) for $K=\partial M \backslash Z$, as $F_{1}(K)+2^{-1} \chi(K)=$ $n \chi(K) / 2$, according to (2.153). The equality (2.169) for $F_{1}\left(M_{\nu}, Z\right)$ follows from its particular cases for $F_{1}\left(M_{j}, Z_{j} \cup N\right)$ by using (2.149) and (2.170).

Corollary 2.10. 1. The analytic torsion $T_{0}\left(M_{\nu}, Z ; m\right)$ (defined by (2.161)) is the following function of $m=[\theta / 2 \pi](\theta \notin 2 \pi \mathbb{Z})$ :

$$
T_{0}\left(M_{\nu}, Z ; m\right)=(-1)^{m n \chi(M, Z)} T_{0}\left(M_{\nu}, Z\right) .
$$

Here $T_{0}\left(M_{\nu}, Z\right):=T_{0}\left(M_{\nu}, Z ; 0\right), n:=\operatorname{dim} M$.

2. The analytic torsion $T_{0}(M, Z ; m)$ is equal to $T\left(M_{\nu_{0}}, Z ; m\right)$ for $\nu_{0}=(1,1)$ according to Proposition 1.1. The formula (2.171) holds also for $T_{0}(M, Z ; m)$, where $T_{0}\left(M_{\nu}, Z\right)$ is replaced by $T_{0}(M, Z):=T_{0}(M, Z ; 0)$.

Let $\left(M, g_{M}\right)$ be obtained by gluing two Riemannian manifolds $M_{1}$ and $M_{2}$ along a common component $N$ of their boundaries, $M=M_{1} \cup_{N} M_{2}$. Let $g_{M}$ be a direct product metric near $N$ and near $\partial M$ and let $Z \subset \partial M$ be a union of some connected components of $\partial M$. The following main theorem is an immediate consequence of Theorems 1.4, 1.5 and of Corollary 2.10.

Theorem 2.2 (Generalized Ray-Singer conjecture). 1. The analytic torsion $T_{0}\left(M_{\nu}, Z ; m\right)$ is expressed through the combinatorial torsion norm (1.62) as follows:

$$
T_{0}\left(M_{\nu}, Z ; m\right)=2^{\chi(\partial M)+\chi(N)}(-1)^{m n \chi(M, Z) / 2} \tau_{0}\left(M_{\nu}, Z\right)
$$

(where $m=[\theta / 2 \pi], \theta \notin 2 \pi \mathbb{Z}$ is the phase of a cut of the spectral plane $\mathbb{C} \ni \lambda$ and $n=\operatorname{dim} M)$.

2. The analytic torsion $T_{0}(M, Z ; m)$ is expressed through the combinatorial torsion norm:

$$
T_{0}(M, Z ; m)=2^{\chi(\partial M)}(-1)^{m n \chi(M, Z) / 2} \tau_{0}(M, Z)
$$

Remark 2.11. The combinatorial torsion norms $\tau_{0}(M, Z)$ and $\tau_{0}\left(M_{\nu_{0}}, Z\right.$ ) (where $\nu_{0}=$ $(1,1))$ on the determinant line $\operatorname{det} H^{\bullet}\left(M_{\nu_{0}}, Z\right)=\operatorname{det} H^{\bullet}(M, Z)$ are different, if $\chi(N) \neq 0$ (by Remarks 1.7 and 1.9). The canonical identifications $H^{\bullet}\left(M_{\nu_{0}}, Z\right)=$ $\operatorname{Ker}\left(\Delta_{\nu_{0}}^{\bullet}\right)=\operatorname{Ker}\left(\Delta^{\bullet}\right)=H^{\bullet}(M, Z)$ are given by Proposition 1.1 and by the de Rham theorem. 


\section{ZETA- AND THETA-FUNCTIONS FOR THE LAPLACIANS WITH $\nu$-TRANSMission INTERIOR BOUNDARY CONDITIONS}

3.1. Properties of zeta- and theta-functions for $\nu$-transmission boundary conditions. Let $M$ be a compact manifold with boundary obtained by gluing manifolds $M_{1}$ and $M_{2}$ along a common component $N$ of their boundaries, $M=M_{1} \cup_{N} M_{2}$ ( $N \subset M$ is a closed codimension one submanifold of $M$ with a trivial normal bundle $\left.\left.T M\right|_{N} / T N\right)$. Let $g_{M}$ be a direct product metric near $N=0 \times N \hookrightarrow I \times N \hookrightarrow M$. Let the Dirichlet boundary conditions be given on a union $Z$ of some connected components of $\partial M$, the Neumann ones be given on $\partial M \backslash Z$ and the $\nu$-transmission interior boundary conditions (1.27) be given on $N$.

The operator $\Delta_{\nu}^{\bullet}$ is originally defined on the set $D\left(\Delta_{\nu}^{\bullet}\right)$ of all the pairs of smooth forms $\omega=\left(\omega_{1}, \omega_{2}\right) \in D R \bullet\left(\bar{M}_{1}\right) \oplus D R \bullet\left(\bar{M}_{2}\right)$ such that the Dirichlet boundary conditions hold for $\omega$ on $Z$, the Neumann boundary conditions hold on $\partial M \backslash Z$ and the interior boundary conditions $(1.27)$ hold for $\omega$ on $N$. Let $\operatorname{Dom}\left(\Delta_{\nu}^{\bullet}\right)$ be the closure of the $D\left(\Delta_{\nu}^{\bullet}\right)$ in $\left(D R^{\bullet}(M)\right)_{2}$ in the topology given by the graph norm ${ }^{47}$ $\|\omega\|_{2}^{2}+\left\|\Delta_{\nu}^{\bullet} \omega\right\|_{2}^{2}=:\|\omega\|_{\text {graph }}^{2}$. The closure of the operator $\Delta_{\nu}^{\bullet}$ (with respect to the graph norm) is an operator with the domain of definition $\operatorname{Dom}\left(\Delta_{\nu}^{\bullet}\right)$. If $\omega_{j} \rightarrow \omega$ in the graph norm topology, $\omega_{j} \in D\left(\Delta_{\nu}^{\bullet}\right)$, then $\Delta_{\nu}^{\bullet}(\omega)$ is defined as $\lim _{j} \Delta_{\nu} \omega_{j}$ in the $L_{2}$-topology in $\left(D R^{\bullet}(M)\right)_{2}$.

Theorem 3.1. 1. The operator $\Delta_{\nu}^{\bullet}$ with the domain $\operatorname{Dom}\left(\Delta_{\nu}^{\bullet}\right)$ is self-adjoint in $\left(D R^{\bullet}(M)\right)_{2}$. Its spectrum $\operatorname{Spec}\left(\Delta_{\nu}^{\bullet}\right) \subset \mathbb{R}_{+} \cup 0$ is discrete. ${ }^{48}$

2. Its zeta-function is defined for $\operatorname{Re} s>(\operatorname{dim} M) / 2$ by the absolutely convergent series (including the multiplicities) $\zeta_{\nu, \bullet}(s):=\sum_{\lambda_{j} \in \operatorname{Spec}\left(\Delta_{\nu}\right) \backslash 0} \lambda_{j}^{-s}$. This series converges uniformly for $\operatorname{Re} s \geq(\operatorname{dim} M) / 2+\varepsilon$ (for an arbitrary $\varepsilon>0)$. The zeta-function $\zeta_{\nu, \bullet}$ can be contunued to a meromorphic function on the whole complex plane with at most simple poles at the points $s_{j}:=(j-\operatorname{dim} M) / 2, j=0,1,2, \ldots$ It is regular at $s=0,1,2, \ldots$

3. The residues $\operatorname{res}_{s=s_{j}} \zeta_{\nu, \bullet}(s)$ and the values $\zeta_{\nu, \bullet}(m)+\delta_{m, 0} \operatorname{dim} \operatorname{Ker}\left(\Delta_{\nu}^{\bullet}\right)$ are equal to the sums of the integrals over $M, \partial M$, and $N$ of the densities locally defined on these manifolds.

Proposition 3.1. 1. Let $\lambda \notin \operatorname{Spec}\left(\Delta_{\nu}^{\bullet}\right)$. Then the resolvent $G_{\lambda}^{\bullet}(\nu):=\left(\Delta_{\nu}^{\bullet}-\lambda\right)^{-1}$, $G_{\lambda}^{\bullet}(\nu):\left(D R^{\bullet}(M)\right)_{2} \underset{\sim}{\sim} \operatorname{Dom}\left(\Delta_{\nu}^{\bullet}\right) \hookrightarrow\left(D R^{\bullet}(M)\right)_{2}$, is the isomorphism (in algebraic and topological senses) onto the closure $\operatorname{Dom}\left(\Delta_{\nu}^{\bullet}\right)$ of $D\left(\Delta_{\nu}^{\bullet}\right)$ with respect to the graph norm. ${ }^{49}$ The operators $G_{\lambda}^{\bullet}(\nu)$ for pairs $(\lambda, \nu)$ such that $\lambda \notin \operatorname{Spec}\left(\Delta_{\nu}^{\bullet}\right)$ form a smooth

\footnotetext{
${ }^{47}$ The $L_{2}$-completion $\left(D R^{\bullet}(M)\right)_{2}$ of $D R^{\bullet}(M)$ coincides with the $L_{2}$-completion of $D R^{\bullet}\left(\bar{M}_{1}\right) \oplus$ $D R^{\bullet}\left(\bar{M}_{2}\right)$.

${ }^{48} \mathrm{~A}$ spectrum is discrete if it consists entirely of isolated eigenvalues with finite multiplicities.

${ }^{49}$ The topology on $\left(D R^{\bullet}(M)\right)_{2}$ is given by $\|\omega\|_{2}^{2}$, and on $\operatorname{Dom}\left(\Delta_{\nu}^{\bullet}\right)$ it is given by $\|\omega\|_{\text {graph }}^{2}$.
} 
in $(\lambda, \nu)$ family of bounded operators in $\left(D R^{\bullet}(M)\right)_{2}$.

2. The families $d \circ G_{\lambda}^{\bullet}(\nu)$ and $\delta \circ G_{\lambda}^{\bullet}(\nu)$ for $\lambda \notin \operatorname{Spec}\left(\Delta_{\nu}^{\bullet}\right)$ form a smooth in $(\lambda, \nu)$ family of bounded operators $\left(D R^{\bullet}(M)\right)_{2} \rightarrow\left(D R^{\bullet \pm 1}(M)\right)_{2}$.

Theorem 3.2. 1. The operator $\exp \left(-t \Delta_{\nu}^{\bullet}\right)$ in $\left(D R^{\bullet}(M)\right)_{2}$ for an arbitrary $t>0$ is of trace class. For its trace the asymptotic expansion (2.87) (relative to $t \rightarrow+0$ ) holds. The coefficients $f_{-\operatorname{dim} M+j}$ of this expansion are the sums of the integrals over $M, \partial M$, and $N$ of the locally defined densities. If $j \neq \operatorname{dim} M+2 m, m \in \mathbb{Z}_{+} \cup 0$, the densities on $M, \partial M$, and on $N$ for $f_{-\operatorname{dim} M+j}$ are the same as for

$$
\Gamma((\operatorname{dim} M-j) / 2) \operatorname{res}_{s=s_{j}} \zeta_{\nu, \bullet}(s) .
$$

If $j=\operatorname{dim} M+2 m, m \in \mathbb{Z}_{+} \cup 0$, these densities are the same as for

$$
(m !)^{-1}(-1)^{m}\left(\zeta_{\nu, \bullet}(m)+\delta_{m, 0} \operatorname{dim} \operatorname{Ker}\left(\Delta_{\nu}^{\bullet}\right)\right)
$$

2. Let $p_{1}:\left(D R^{\bullet}(M)\right)_{2} \rightarrow\left(D R^{\bullet}\left(M_{1}\right)\right)_{2} \hookrightarrow\left(D R^{\bullet}(M)\right)_{2}$ be the composition of the restriction to $M_{1}$ and of the extension by zero of $L_{2}$-forms. Then the operator $p_{1} \exp \left(-t \Delta_{\nu}^{\bullet}\right)$ in $\left(D R^{\bullet}(M)\right)_{2}$ for $t>0$ is of trace class. For its trace the asymptotic expansion relative to $t \rightarrow+0$ holds

$$
\operatorname{Tr}\left(p_{1} \exp \left(-t \Delta_{\nu}^{\bullet}\right)\right)=q_{-n} t^{-n / 2}+\cdots+q_{0} t^{0}+q_{1} t^{1 / 2}+\cdots+q_{m} t^{m / 2}+r_{m}(t),
$$

where $r_{m}(t)$ is $O\left(t^{(m+1) / 2}\right)$ uniformly with respect to $\nu$ and it is smooth in $t$ for $t>0$ $(n:=\operatorname{dim} M)$. The coefficients $q_{j}$ are equal to the sums of the integrals over $M_{1}$ and over $\partial M_{1}=N \cup\left(\partial M \cap \bar{M}_{1}\right)$ of the locally defined densities. The coefficients $q_{j}$ in (3.1) depend only on $\left(j, M_{1},\left.g_{M}\right|_{T M_{1}}, Z \cap \partial \bar{M}_{1}, N, \nu\right)$ and do not depend on $M_{2}$ and $Z \cap \partial \bar{M}_{2},\left.g_{M}\right|_{T M_{2}}$.

3. For any $t>0$ the traces of $\exp \left(-t \Delta_{\nu}^{\bullet}\right)$ are bounded uniformly with respect to $\nu \in \mathbb{R}^{2} \backslash(0,0)$ :

$$
\left|\operatorname{Tr} \exp \left(-t \Delta_{\nu}^{\bullet}\right)\right|<C(t)
$$

The traces $\operatorname{Tr}\left(p_{j} \exp \left(-t \Delta_{\nu}^{\bullet}\right)\right)$ are also bounded uniformly with respect to $\nu$ for any $t>0$.

Proposition 3.2. 1. The kernel $E_{t, x_{1}, x_{2}}^{\bullet}(\nu)$ for $\exp \left(-t \Delta_{\nu}^{\bullet}\right)$ (where $\left.t>0\right)$ is smooth in $x_{j} \in \bar{M}_{r_{j}}$, , and in $\nu \in \mathbb{R}^{2} \backslash(0,0)$.

2. The asymptotic expansions (3.1), (2.87) are differentiable with respect to $\nu \in$ $\mathbb{R}^{2} \backslash(0,0)$. 
3.2. Zeta-functions for the Laplacians with $\nu$-transmission interior boundary conditions. Proofs of Theorem 3.1 and of Proposition 3.1. Let $A$ be an elliptic differential operator on a manifold with boundary $(M, \partial M)$. Let the differential elliptic boundary conditions be given for $A$ on $\partial M$ such that $A$ with these boundary conditions satisfies Agmon's condition (formulated below) for $\lambda$ from a sector $\theta_{1}<\arg \lambda<\theta_{2}$ in the spectral plane $\mathbb{C} \ni \lambda$. Properties of zeta-functions for $A$ with these boundary conditions can be investigated with the help of the parametrix for $(A-\lambda)^{-1}$. The analogous statement is true also for elliptic interior boundary conditions. ${ }^{50}$ The parametrix $P_{\lambda}^{m}$ for $\left(\Delta_{\nu}^{\bullet}-\lambda\right)^{-1}$ is defined locally in coordinate charts. Namely

$$
P_{\lambda}^{m}=\sum \psi_{j} P_{\lambda, U_{j}}^{m} \varphi_{j}
$$

where $\varphi_{j}$ is a partition of unity subordinate to a finite cover $\left\{U_{j}\right\}$ of $M$ by coordinate charts, $\psi_{j} \varphi_{j}=\varphi_{j}, \psi_{j} \in C_{0}^{\infty}\left(U_{j}\right)$. If $U_{j} \cap(\partial M \cup N)=\emptyset$ then the operator $P_{\lambda, U_{j}}^{m}$ is a pseudodifferential operator (PDO) with parameter $\lambda$ ([Sh], Chapter II, $\S 9$ ) and its symbol is equal to $\theta(\xi, \lambda) s_{(m)}\left(\left(\Delta^{\bullet}-\lambda\right)^{-1}\right)(x, \xi, \lambda)$. This symbol is defined as follows. Let $s\left(\Delta^{\bullet}-\lambda\right)=\left(\left(b_{2}-\lambda\right) \mathrm{id}+b_{1}\right)(x, \xi)$ be the symbol of $\Delta^{\bullet}-\lambda$ (where $\Delta^{\bullet}$ is the Laplacian on $\left.D R^{\bullet}\left(U_{j}\right)\right)$ and let

$$
s\left(\left(\Delta^{\bullet}-\lambda\right)^{-1}\right):=\sum_{j \in \mathbb{Z}_{+} \cup 0} a_{-2-j}(x, \xi, \lambda)
$$

be the symbol of $\left(\Delta^{\bullet}-\lambda\right)^{-1}$ as of a PDO with parameter $\left(a_{-k}\right.$ is positive homogeneous of degree $-k$ in $\left.\left(\xi, \lambda^{1 / 2}\right)\right)$. Set $s_{(m)}\left(\left(\Delta^{\bullet}-\lambda\right)^{-1}\right):=\sum_{j=0}^{m} a_{-2-j}(x, \xi, \lambda)$. The condition $s\left(\Delta^{\bullet}-\lambda\right) \circ s\left(\left(\Delta^{\bullet}-\lambda\right)^{-1}\right)=1$, where $\circ$ is the composition of symbols with parameter $([\mathrm{Sh}], \S 11.1)$, is equivalent to the system of equalities

$$
\begin{gathered}
a_{-2}(x, \xi, \lambda)=\left(b_{2}-\lambda\right)^{-1}, \\
a_{-3}=-\left(b_{2}-\lambda\right)^{-1}\left[b_{1} a_{-2}+\sum_{i} D_{\xi_{i}} b_{2} \partial_{x_{i}} a_{-2}\right], \\
a_{-2-j}=-\left(b_{2}-\lambda\right)^{-1} \sum_{|\gamma|+i+l=j} \frac{1}{\gamma !} D_{\xi}^{\gamma} b_{2-i} \partial_{x}^{\gamma} a_{-2-l} .
\end{gathered}
$$

The sum in the last equation of (3.4) is over $(\gamma, i, l)$ such that $\gamma=\left(\gamma_{1}, \ldots, \gamma_{n}\right) \in$ $\left(\mathbb{Z}_{+} \cup 0\right)^{n},|\gamma|:=\gamma_{1}+\cdots+\gamma_{n}, 0 \leq|\gamma| \leq j$ for $b_{j},|\gamma|+i \geq 1\left(D:=i^{-1} \partial\right)$. The function $\theta(\xi, \lambda)$ (in the symbol of $P_{\lambda}^{m}$ ) is smooth, $\theta(\xi, \lambda) \equiv 1$ for $|\xi|^{2}+|\lambda| \geq 1$, and $\theta$ is equal to zero for $|\xi|^{2}+|\lambda| \leq \varepsilon$.

\footnotetext{
${ }^{50}$ Theorem 3.1 is analogous to the results of [Se1], [Se2] with modifications connected with the $\nu$ transmission interior boundary conditions. In [Sh], Ch.II, the theory [Se1], [Se2] of the zeta-functions is written in detail in the case of a closed manifold.
} 
Let $U_{j} \cap N \neq \emptyset$. Then the term $P_{\lambda, U}^{m}$ of the parametrix is the sum of the interior term (which is a PDO with parameter and its symbol is defined with the help of (3.4)) and of the correction terms. (Here $U:=U_{j}$.) The latter terms correspond to the $\nu$-transmission interior boundary conditions on $N$ and to the Dirichlet and the Neumann boundary conditions, given on the connected components of $\partial M$. First of all we'll verify that these $\nu$-transmission boundary conditions are Agmon's conditions on any ray $\arg \lambda=\varphi$ in the spectral plane not coinciding with $\mathbb{R}_{+}$.

Let $(t, y) \in I \times U_{N}$ be the coordinates on $U:=U_{j}$ near $N=0 \times N \hookrightarrow I \times N \hookrightarrow M$, $I=[-2,2]$, and let $t>0$ on $M_{1}$. From now on it is supposed that $\varphi_{j}(t, y)=$ $\varphi_{j, I}(t) \varphi_{j, N}(y)$ and that $\varphi_{j, I}(t) \equiv 1$ for $|t| \leq 1$. It is supposed also that $\psi_{j}(t, y)=$ $\psi_{j, I}(t) \psi_{j, N}(y)$ and that $\varphi_{j, I}, \psi_{j, I}$ are even functions: $\varphi_{j, I}(-t)=\varphi_{j, I}(t), \psi_{j, I}(-t)=$ $\psi_{j, I}(t)$. The forms $d y^{c}$ and $d t d y^{f}$ (where $c=\left(c_{1}, \ldots, c_{n-1}\right), f=\left(f_{1}, \ldots, f_{n-1}\right), c_{i}, f_{i} \in$ $\{0,1\})$ provide us with a trivialization of $\left.\wedge^{\bullet} T M\right|_{I \times U_{N}}$. Namely $\omega_{j}=\sum_{|c|=\bullet} \omega_{j, c} d y^{c}+$ $\sum_{|f|+1=\bullet} \omega_{j,(1, f)} d t d y^{f}$. Let $\omega=\left(\omega_{1}, \omega_{2}\right) \in D\left(\Delta_{\nu}^{\bullet}\right) \subset D R^{\bullet}\left(M_{\nu}\right)$. Then on $U_{N}=$ $0 \times U_{N} \hookrightarrow I \times U_{N}$ the conditions $\omega \in D\left(\Delta_{\nu}^{\bullet}\right)$ can be written as follows. Set $|\nu|=$ $\left(\alpha^{2}+\beta^{2}\right)^{1 / 2}$. Let $\mathcal{L}$ be the transformation $(t \geq 0)$

$$
\begin{aligned}
v_{1, c}(t, y) & :=|\nu|^{-1}\left(\alpha \omega_{1, c}(t, y)-\beta \omega_{2, c}(-t, y)\right), \\
v_{2, c}(t, y) & :=|\nu|^{-1}\left(\beta \omega_{1, c}(t, y)+\alpha \omega_{2, c}(-t, y)\right), \\
w_{2,(1, f)}(t, y) & :=|\nu|^{-1}\left(\alpha \omega_{1,(1, f)}(t, y)+\beta \omega_{2,(1, f)}(-t, y)\right), \\
w_{1,(1, f)}(t, y) & :=|\nu|^{-1}\left(-\beta \omega_{1,(1, f)}(t, y)+\alpha \omega_{2,(1, f)}(-t, y)\right) .
\end{aligned}
$$

Then the conditions $\omega \in D\left(\Delta_{\nu}^{\bullet}\right)$ are equivalent on $U_{N}$ to

$$
\begin{array}{ll}
v_{1, c}(0, y)=0, & w_{1,(1, f)}(0, y)=0, \\
\left.\partial_{t} v_{2, c}\right|_{t=0}=0, & \left.\partial_{t} w_{2,(1, f)}\right|_{t=0}=0 .
\end{array}
$$

The inverse to (3.5) transformation $\mathcal{L}^{-1}$ is

$$
\begin{gathered}
\left(\begin{array}{c}
\omega_{1, c}(t, y) \\
\omega_{2, c}(-t, y)
\end{array}\right)=L^{-1}\left(\begin{array}{c}
v_{1, c} \\
v_{2, c}
\end{array}\right)(t, y),\left(\begin{array}{c}
\omega_{2,(1, f)}(-t, y) \\
\omega_{1,(1, f)}(t, y)
\end{array}\right)=L^{-1}\left(\begin{array}{c}
w_{1,(1, f)} \\
w_{2,(1, f)}
\end{array}\right)(t, y), \\
L:=|\nu|^{-1}\left(\begin{array}{cc}
\alpha & -\beta \\
\beta & \alpha
\end{array}\right) .
\end{gathered}
$$

Agmon's conditions on a ray $l:=\{\arg \lambda=\varphi\}$ in the case of $\nu$-transmission boundary conditions claim that for $\left(\xi^{\prime}, \lambda\right) \neq(0,0)$ and $\lambda \in l$ the equation on $\mathbb{R}_{+} \ni t$

$$
\left(-\partial_{t}^{2}+b_{2}\left(y, \xi^{\prime}\right)-\lambda\right) v(t)=0, \quad v(t) \rightarrow 0 \text { for } t \rightarrow+\infty
$$

has a unique solution for each of the initial conditions $v_{t=0}=v_{0}$ or $\left.\partial_{t} v\right|_{t=0}=v_{1}$. (Here $\xi^{\prime}$ are dual to $y$ and $b_{2}\left(\Delta_{N}\right)=b_{2}\left(y, \xi^{\prime}\right)$ id is the scalar principal symbol of $\Delta_{N}^{\bullet}$ on $U_{N}$.) Agmon's conditions for the $\nu$-transmission boundary value problem are 
satisfied on each ray $\arg \lambda=\varphi$ not coinciding with $\mathbb{R}_{+}$because the equation (3.8) with each of the initial conditions given above has a unique solution for any $\lambda \notin \mathbb{R}_{+}$, $\left(\xi^{\prime}, \lambda\right) \neq(0,0)$.

It is convenient to compute the contribution to $P_{\lambda, U}^{m}$ from $\nu$-transmission boundary conditions in the coordinates $v_{j, c}(t, y), w_{j,(1, f)}(t, y)$ defined by (3.5) with $t \geq$ 0 . (Then the $\nu$-transmission boundary conditions are transformed into the conditions (3.6).) These contributions are defined with the help of the symbol $d=$ $\sum_{j \in \mathbb{Z}_{+} \cup 0} d_{-2-j}\left(t, y, \tau, \xi^{\prime}, \lambda\right)$, which is the solution of the equation ${ }^{51}$

$$
\left(-\partial_{t}^{2}+\left(b_{2}\left(y, \xi^{\prime}\right)-\lambda\right) \mathrm{id}+b_{1}\left(y, \xi^{\prime}\right)\right) \circ d\left(t, y, \tau, \xi^{\prime}, \lambda\right)=0
$$

(with the composition $\circ$ of symbols of $\left(y, \xi^{\prime}\right)$ in it). The equation (3.9) holds for $t \neq 0$. The boundary conditions for (3.9) are: $d_{-k} \rightarrow 0$ as $|t| \rightarrow \infty$ and

$$
\begin{aligned}
\left.\left(\mathcal{L} d_{-k}\right)_{1, c}\right|_{t=0} & =\left.\left(\mathcal{L} a_{-k}\right)_{1, c}\right|_{t=0},\left.\quad\left(\mathcal{L} d_{-k}\right)_{1,(1, f)}\right|_{t=0}=\left.\left(\mathcal{L} a_{-k}\right)_{1,(1, f)}\right|_{t=0}, \\
\left.\partial_{t}\left(\mathcal{L} d_{-k}\right)_{2, c}\right|_{t=0} & =\left.i \tau\left(\mathcal{L} a_{-k}\right)_{2, c}\right|_{t=0},\left.\partial_{t}\left(\mathcal{L} d_{-k}\right)_{2,(1, f)}\right|_{t=0}=\left.i \tau\left(\mathcal{L} a_{-k}\right)_{2,(1, f)}\right|_{t=0}
\end{aligned}
$$

Here the transformation $\mathcal{L}$ acts on the columns of the matrix valued functions $d$, $a$ (depending on $t$ and on $\tau) .^{52}$

The equation (3.9) is the recurrent system

$$
-\partial_{t}^{2} d_{-k}+\left(b_{2}-\lambda\right) d_{-k}+\sum \frac{1}{\gamma !} D_{\xi^{\prime}}^{\gamma} b_{i} \partial_{y}^{\gamma} d_{-m}=0,
$$

where the sum is over $m<k$ and $\gamma$ such that $m+|\gamma|+2-i=k, 0 \leq|\gamma| \leq i$ for $b_{i}$.

For $t=0$ the symbol $d_{-k}$ over $M_{j} \cap U_{N}$ is positive homogeneous of degree $(-k)$ in $\left(\tau, \xi^{\prime}, \lambda^{1 / 2}\right)$. The boundary contribution to $P_{\lambda, U}^{m}$ is an operator $\mathcal{D}_{m}$ corresponding to $^{53} \theta_{1}(\xi, \lambda) \sum_{j=0}^{m} d_{-2-j}\left(t, y, \tau, \xi^{\prime}, \lambda\right)$. This operator acts on $f \in D R_{c}^{\bullet}\left(\mathbb{R}_{t} \times \mathbb{R}_{y}^{n-1}\right)$ such that supp $f \cap\left(0 \times \mathbb{R}_{y}^{n-1}\right)=\emptyset$ as follows:

$$
\left(\mathcal{D}_{m} f\right)(y, t)=(2 \pi)^{-n} \iint \exp \left(i\left(y, \xi^{\prime}\right)\right) \sum_{0}^{m} \theta_{1} d_{-2-j}\left(t, y, \tau, \xi^{\prime}, \lambda\right)(\mathcal{F} f)\left(\tau, \xi^{\prime}\right) d \xi^{\prime} d \tau
$$

(where $(\mathcal{F} f)\left(\tau, \xi^{\prime}\right)=\iint \exp \left(-i\left(t \tau+\left(x, \xi^{\prime}\right)\right)\right) f(t, x) d x d t$ is Fourier transform of $\left.f\right)$. The term of the parametrix, corresponding to $U$ (if $U \cap N \neq \emptyset$ ) is defined by

$$
P_{\lambda, U}^{m}=P_{\lambda, i n t}^{m}-\mathcal{D}_{m}
$$

\footnotetext{
${ }^{51}$ Here $b_{2}\left(y, \xi^{\prime}\right) \mathrm{id}+b_{1}(y, \xi)$ is the symbol $s\left(\Delta_{N}^{\bullet}\right)$ on $U_{N}$ of the Laplacian on $D R^{\bullet}(N)$ for the components $\omega_{\text {tan,N }}(t, y)$ and on $D R^{\bullet-1}(N)$ for $\omega_{\text {norm }, N}(t, y)$. The variable $\tau$ is dual to $t$.

${ }^{52}$ Note that the function $a_{-2-j}\left(t, y, \tau, \xi^{\prime}, \lambda\right)$ is continuous in $N$ and nonsingular for $\lambda \notin \mathbb{R}_{+}$and $\left(\tau, \xi^{\prime}, \lambda\right) \neq(0,0,0)$. (It is also independent of $t$ for $|t|$ small enough.) So the right sides of (3.10) can be simplified for $a_{-k}$. In (3.9)-(3.11) it is used that $g_{M}$ is a direct product metric on $I \times N \hookrightarrow M$.

${ }^{53} \theta_{1}\left(\xi^{\prime}, \lambda\right) \in C^{\infty}\left(\mathbb{R}^{n-1} \times \mathbb{C}\right), \theta_{1} \equiv 0$ for $\left|\xi^{\prime}\right|^{2}+|\lambda|<\varepsilon$ and $\theta_{1} \equiv 1$ for $\left|\xi^{\prime}\right|^{2}+|\lambda| \geq 1 ; n:=\operatorname{dim} M$
} 
where $P_{\lambda, \text { int }}$ is the PDO with the symbol $s_{(m)}\left(t, y, \tau, \xi^{\prime}, \lambda\right)$ defined by $(3.4)$ ( $x$ is replaced by $(t, y)$ and $\left.\xi=\left(\tau, \xi^{\prime}\right)\right)$.

For $U_{j} \cap \partial M \neq \emptyset$ the boundary term in $P_{\lambda, U_{j}}^{m}$ for the Dirichlet or the Neumann boundary conditions on the connected components of $\partial M$ is defined similarly.

The following assertions are true:

1. For $m \geq n$ the operator $\left(\Delta^{\bullet}-\lambda\right) P_{\lambda}^{m}-$ id (where $\left(\Delta^{\bullet}-\lambda\right)$ acts on the restrictions of forms to $\bar{M}_{1}$ and to $\bar{M}_{2}$ ) has a continuous on $\bar{M}_{j_{1}} \times \bar{M}_{j_{2}}$ kernel which is $O\left(\left(1+|\lambda|^{1 / 2}\right)^{n-m}\right)$ for $\lambda \in \Lambda_{\varepsilon}:=\{\lambda \neq 0, \varepsilon<\arg \lambda<2 \pi-\varepsilon\}$, where $\pi>\varepsilon>0$ is fixed ([Se1], Lemma 5, p. 901). This estimate is satisfied uniformly with respect to $\nu$ since the families $d_{-2-j}$ are smooth in $\nu \in \mathbb{R}^{2} \backslash(0,0)$ and since the estimates for $\mathcal{L} d_{-2-j}$ by [Se1], (29), p. 900, are uniform with respect to $\nu \neq(0,0)$.

2. Let $m \geq n$ and $\nu=(\alpha, \beta) \in \mathbb{R}^{2} \backslash(0,0)$. Let $A_{j}:=A\left(M_{j}, N\right)$ be the same as in (2.97) and (2.99) and $R_{j}$ be the geometrical restrictions to $N \subset \partial M_{j}$ of forms on $M_{j}$. Then the operators

$$
\begin{array}{cc}
|\nu|^{-1}\left(\alpha R_{1}-\beta R_{2}\right) P_{\lambda}^{m}, & |\nu|^{-1}\left(\beta A_{1}-\alpha A_{2}\right) P_{\lambda}^{m}, \\
|\nu|^{-1}\left(\alpha R_{1} \delta-\beta R_{2} \delta\right) P_{\lambda}^{m}, & |\nu|^{-1}\left(\beta A_{1} d-\alpha A_{2} d\right) P_{\lambda}^{m}
\end{array}
$$

have smooth kernels on $N \times \bar{M}_{j}$ which are $O\left(\left(1+|\lambda|^{1 / 2}\right)^{n-m}\right)$ for $\lambda \in \Lambda_{\varepsilon}$, where $\pi>\varepsilon>0$ and $\varepsilon$ is fixed ([Se1], Lemma 6). These estimates are uniform with respect to $\nu \in \mathbb{R}^{2} \backslash(0,0)$.

3. Set $B_{1, \nu}:=|\nu|^{-1}\left(\alpha R_{1}-\beta R_{2}\right): \oplus_{j} D R^{\bullet}\left(\bar{M}_{j}\right) \rightarrow D R^{\bullet}(N)$. Let $p_{1}:[0,1] \times N \rightarrow N$, $p_{2}:[-1,0] \times N \rightarrow N$ be the natural projections. The operator

$$
q_{1, \nu}: D R^{\bullet}(N) \rightarrow \oplus D R^{\bullet}\left(M_{j}\right), \quad q_{1, \nu}\left(\omega_{N}\right):=|\nu|^{-1} \varphi(t)\left(\alpha p_{1}^{*} \omega_{N},-\beta p_{2}^{*} \omega_{N}\right)
$$

(where $\varphi(t) \in C_{0}^{\infty}(I), \varphi(t) \equiv 1$ for $t \in[-1 / 2,1 / 2]$ ) is the right inverse to $B_{1, \nu}$ since $B_{1, \nu} q_{1, \nu}=\mathrm{id}$. The analogous right inverse operators $q_{k, \nu}$ are naturally defined for $B_{k, \nu}$

$$
\begin{gathered}
B_{2, \nu}:=|\nu|^{-1}\left(\beta A_{1}-\alpha A_{2}\right), \quad B_{3, \nu}:=|\nu|^{-1}\left(\alpha R_{1} \delta-\beta R_{2} \delta\right) \\
B_{4, \nu}:=|\nu|^{-1}\left(\beta A_{1} d-\alpha A_{2} d\right) \\
B_{i, \nu} q_{j, \nu}=\delta_{i j} \cdot \mathrm{id} .
\end{gathered}
$$


For instance, $q_{3, \nu} \circ \omega_{N}:=\varepsilon|\nu|^{-1} \varphi(t) t\left(\alpha d t \wedge p_{1}^{*} \omega_{N},-\beta d t \wedge p_{2}^{*} \omega_{N}\right), \varepsilon= \pm 1$. Set $B_{\nu}:=$ $\left(B_{j, \nu}\right), q_{\nu}:=\left(q_{j, \nu}\right)$, and $q_{\nu} B_{\nu}:=\left(q_{j, \nu} B_{j, \nu}\right)$. For $m \geq n$ the operator is defined ${ }^{54}$

$$
R_{\lambda}^{m}:=P_{\lambda}^{m}-q_{\nu} B_{\nu} P_{\lambda}^{m}
$$

It maps (according to [Se1], Lemma 12, p. 912) $C^{\infty}$-forms $\omega \in D R^{\bullet}(M), \operatorname{supp} \omega \cap N=$ $\emptyset$, to $C^{\infty}$-forms on $M_{j}$. Moreover $R_{\lambda}^{m}: D R_{c}^{\bullet}(M \backslash N) \rightarrow D\left(\Delta_{\nu}^{\bullet}\right)$.

4. For $m \geq n$ and $\lambda \notin \mathbb{R}_{+} \cup 0$ the operator $R_{\lambda}$ can be continued to a bounded operator in $\left(D R^{\bullet}(M)\right)_{2}, R_{\lambda}:\left(D R^{\bullet}(M)\right)_{2} \rightarrow \operatorname{Dom}\left(\Delta_{\nu}^{\bullet}\right)$.

Indeed, for any fixed differential operator $F$ of order $d=d(F) \leq 2$ the operator $F R_{\lambda}$ is bounded in $\left(D R^{\bullet}(M)\right)_{2}$ with its norm $O\left(\left(1+|\lambda|^{1 / 2}\right)^{d-2}\right)$ in a sector $\lambda \in \Lambda_{\varepsilon}$ $(\pi>\varepsilon>0$ and $\varepsilon$ is fixed) according to [Se1], Lemmas $7,13,14$. This estimate is uniform with respect to $\nu \in \mathbb{R}^{2} \backslash(0,0)$. The continuation of $R_{\lambda}$ to $\left(D R^{\bullet}(M)\right)_{2}$ is as follows. If $\omega_{j} \in D R_{c}^{\bullet}(M \backslash N)$ and $\omega_{j} \rightarrow \omega$ in $\left(D R^{\bullet}(M)\right)_{2}$ then $R_{\lambda} \omega_{j}$ converges in $\left(D R^{\bullet}(M)\right)_{2}$ and $R_{\lambda} \omega$ is defined as its limit. We see that $R_{\lambda} \omega_{j} \in D\left(\Delta_{\nu}^{\bullet}\right)$ and $\left(\Delta_{\nu}^{\bullet}-\lambda\right) R_{\lambda} \omega_{j}$ converges in $\left(D R^{\bullet}(M)\right)_{2}$. Hence $R_{\lambda} \omega \in \operatorname{Dom}\left(\Delta_{\nu}^{\bullet}\right)$.

5. The operator $G_{\lambda}^{\bullet}(\nu):=\left(\Delta_{\nu}^{\bullet}-\lambda\right)^{-1}:\left(D R^{\bullet}(M)\right)_{2} \rightarrow \operatorname{Dom}\left(\Delta_{\nu}^{\bullet}\right) \operatorname{exists}^{55}$ for $\lambda \in \Lambda_{\varepsilon}:=\{\lambda \neq 0: \varepsilon<\arg \lambda<2 \pi-\varepsilon\}$ and $|\lambda|$ sufficiently large. Its operator norm is $O\left(|\lambda|^{-1}\right)$ for such $\lambda$ uniformly with respect to $\nu \in \mathbb{R}^{2} \backslash(0,0)$ ([Se1], Lemma 15).

6. The Laplacian $\Delta_{\nu}^{\bullet}$ is a closed unbounded operator in $\left(D R^{\bullet}(M)\right)_{2}$ with its domain of definition $\operatorname{Dom}\left(\Delta_{\nu}^{\bullet}\right)$. Actually, if $\left\{u_{i}\right\} \subset \operatorname{Dom}\left(\Delta_{\nu}^{\bullet}\right)$ and if the limits $\lim _{i} u_{i}=: u$ and $\lim _{i}\left(\left(\Delta_{\nu}^{\bullet}-\lambda\right) u_{i}\right)=: v$ exist in $\left(D R^{\bullet}(M)\right)_{2}$ then for sufficiently large $\lambda \in \Lambda_{\varepsilon}$ we have $u=\lim _{i} G_{\lambda}^{\bullet}(\nu)\left(\left(\Delta_{\nu}^{\bullet}-\lambda\right) u_{i}\right)=G_{\lambda}(\nu) v \in \operatorname{Dom}\left(\Delta_{\nu}^{\bullet}\right)$. Hence $\left(\Delta_{\nu}^{\bullet}-\lambda\right) u=\left(\Delta_{\nu}^{\bullet}-\lambda\right)\left(G_{\lambda}^{\bullet}(\nu) v\right)=v$, i.e., the operators $\Delta_{\nu}^{\bullet}-\lambda$ id and $\Delta_{\nu}^{\bullet}$ are closed in $\left(D R^{\bullet}(M)\right)_{2}$. The operator $\Delta_{\nu}^{\bullet}$ is defined on $\operatorname{Dom}\left(\Delta_{\nu}^{\bullet}\right)$. It is a self-adjoint unbounded operator in $\left(D R^{\bullet}(M)\right)_{2}$. Indeed, the domain of definition Dom $\left(\left(\Delta_{\nu}^{\bullet}-\lambda\right)^{*}\right)$ of the adjoint operator $\left(\Delta_{\nu}^{\bullet}-\lambda\right)^{*}$ in $\left(D R^{\bullet}(M)\right)_{2}$ is the set of $v \in\left(D R^{\bullet}(M)\right)_{2}$ such that the linear functional $\left(\left(\Delta_{\nu}^{\bullet}-\lambda\right) \omega, v\right)$ is continuous on $\operatorname{Dom}\left(\Delta_{\nu}^{\bullet}\right) \ni \omega$ in the $L_{2^{-}}$ topology of $\left(D R^{\bullet}(M)\right)_{2}$. If $v \in \operatorname{Dom}\left(\Delta_{\nu}^{\bullet}\right)$ then for any $\omega \in \operatorname{Dom}\left(\Delta_{\nu}^{\bullet}\right)$ we have $\left(\left(\Delta_{\nu}^{\bullet}-\lambda_{0}\right) \omega, v\right)=\left(\omega,\left(\Delta_{\nu}^{\bullet}-\lambda_{0}\right) v\right)$ for $\lambda_{0} \in \mathbb{R}_{-}$. Indeed, for each $\omega$ and $v$ from $\operatorname{Dom}\left(\Delta_{\nu}^{\bullet}\right)$ there exist sequences $\left\{\omega_{j}\right\}$ and $\left\{v_{j}\right\}$ of elements $D\left(\Delta_{\nu}^{\bullet}\right)$ whose limits in the graph norm topology are $\omega$ and $v$. Hence we have

$$
\lim _{j}\left(\Delta_{\nu} \omega_{j}, v\right)_{2}=\lim _{j} \lim _{i}\left(\Delta_{\nu} \omega_{j}, v_{i}\right)_{2}=\lim _{j} \lim _{i}\left(\omega_{j}, \Delta_{\nu} v_{i}\right)_{2}=\left(\omega, \Delta_{\nu} v\right)_{2} .
$$

\footnotetext{
${ }^{54}$ For simplicity we'll suppose from now on here that $\partial M=\emptyset$. For the Dirichlet or the Neumann boundary conditions on the components of $\partial M$ the appropriate terms have to be added to $R_{\lambda}^{m}$.

${ }^{55}$ This means that $\Delta_{\nu}^{\bullet}-\lambda$ maps $\operatorname{Dom}^{\bullet}\left(\Delta_{\nu}\right)$ one-to-one to $\left(D R^{\bullet}(M)\right)_{2}$. It is equivalent to the existence of $\left(\Delta_{\nu}^{\bullet}-\lambda\right)^{-1}:\left(D R^{\bullet}(M)\right)_{2} \rightarrow \operatorname{Dom}\left(\Delta_{\nu}^{\bullet}\right),\left(\Delta_{\nu}^{\bullet}-\lambda\right) \circ\left(\Delta_{\nu}^{\bullet}-\lambda\right)^{-1}=\mathrm{id}$ on $\left(D R^{\bullet}(M)\right)_{2}$.
} 
So $\left(\left(\Delta_{\nu}^{\bullet}-\lambda_{0}\right) u, v\right)$ is a continuous linear functional on $\operatorname{Dom}\left(\Delta_{\nu}^{\bullet}\right) \ni u$ with respect to the $L_{2}$-topology of $\left(D R^{\bullet}(M)\right)_{2}$ for any $v \in \operatorname{Dom}\left(\Delta_{\nu}^{\bullet}\right)$. Hence $\operatorname{Dom}\left(\Delta_{\nu}^{\bullet}\right) \subset$ $\operatorname{Dom}\left(\left(\Delta_{\nu}^{\bullet}-\lambda_{0}\right)^{*}\right)$ and $\left(\Delta_{\nu}-\lambda_{0}\right)^{*} v=\left(\Delta_{\nu}-\lambda_{0}\right) v$ for $v \in \operatorname{Dom}\left(\Delta_{\nu}^{\bullet}\right)$.

Let $\lambda_{0} \in \mathbb{R}_{-}$and $\left|\lambda_{0}\right|$ be sufficiently large. Then for any $w \in \operatorname{Dom}\left(\left(\Delta_{\nu}^{\bullet}-\lambda_{0}\right)^{*}\right)$ there exists an element $v \in \operatorname{Dom}\left(\Delta_{\nu}^{\bullet}\right)$ such that $\left(\Delta_{\nu}^{\bullet}-\lambda_{0}\right)^{*} w=\left(\Delta_{\nu}^{\bullet}-\lambda_{0}\right) v=$ $\left(\Delta_{\nu}^{\bullet}-\lambda_{0}\right)^{*} v\left(\right.$ since $\left.\operatorname{Im}\left(\Delta_{\nu}^{\bullet}-\lambda_{0}\right)=\left(D R^{\bullet}(M)\right)_{2}\right)$. So $w-v \in \operatorname{Ker}\left(\left(\Delta_{\nu}^{\bullet}-\lambda_{0}\right)^{*}\right)$ and for any $u \in \operatorname{Dom}\left(\Delta_{\nu}^{\bullet}\right)$ we have $0=\left(u,\left(\Delta_{\nu}^{\bullet}-\lambda_{0}\right)^{*}(w-v)\right)=\left(\left(\Delta_{\nu}^{\bullet}-\lambda_{0}\right) u, w-v\right)$. Then $w-v=0$, as $\operatorname{Im}\left(\Delta_{\nu}^{\bullet}-\lambda_{0}\right)=\left(D R^{\bullet}(M)\right)_{2}$. Hence $\operatorname{Dom}\left(\left(\Delta_{\nu}^{\bullet}-\lambda_{0}\right)^{*}\right)=\operatorname{Dom}\left(\Delta_{\nu}^{\bullet}\right)=$ $\operatorname{Dom}\left(\left(\Delta_{\nu}^{\bullet}\right)^{*}\right)$, and $\Delta_{\nu}^{\bullet}$ is a self-adjoint unbounded operator in $\left(D R^{\bullet}(M)\right)_{2}$.

The operator $\Delta_{\nu}^{\bullet}$ is nonnegative, $\left(\Delta_{\nu}^{\bullet} \omega, \omega\right)_{2} \geq 0$ for any $\omega \in \operatorname{Dom}\left(\Delta_{\nu}^{\bullet}\right)$, since there exists a sequence $\left\{\omega_{j}\right\}, \omega_{j} \in D\left(\Delta_{\nu}^{\bullet}\right)$, such that its limit in the graph norm topology is $\omega$. So we have $\lim _{j}\left(\Delta_{\nu}^{\bullet} \omega_{j}, \omega_{j}\right)_{2}=\lim _{j}\left(d_{\nu} \omega_{j}, d_{\nu} \omega_{j}\right)_{2}+\lim _{j}\left(\delta_{\nu} \omega_{j}, \delta_{\nu} \omega_{j}\right)_{2} \geq 0$.

7. The spectrum $\operatorname{Spec}\left(\Delta_{\nu}^{\bullet}\right)$ of the operator $\Delta_{\nu}^{\bullet}$ is discrete because the operator

$$
\left(\Delta_{\nu}^{\bullet}-\lambda\right)\left(\Delta_{\nu}^{\bullet}-\lambda_{0}\right)^{-1}=\mathrm{id}+\left(\Delta_{\nu}^{\bullet}-\lambda_{0}\right)^{-1} \cdot\left(\lambda_{0}-\lambda\right)
$$

differs from the identity operator in $\left(D R^{\bullet}(M)\right)_{2}$ by a compact operator. Here, $\lambda_{0} \in \Lambda_{\varepsilon}$ and $\left|\lambda_{0}\right|$ is large enough. The assertion 5 above claims that $\left(\Delta_{\nu}^{\bullet}-\lambda_{0}\right)^{-1}$ exists for such $\lambda_{0}$. The operator $G_{\lambda_{0}}(\nu):=\left(\Delta_{\nu}^{\bullet}-\lambda_{0}\right)^{-1}$ is compact since it is bounded in $\left(D R^{\bullet}(M)\right)_{2}$ and since the operators $I-\left(\Delta_{\nu}^{\bullet}-\lambda_{0}\right) R_{\lambda_{0}}^{m}$ (for $m \geq n$ ) and $R_{\lambda_{0}}^{m}$ are compact in $\left(D R^{\bullet}(M)\right)_{2}$ ([Se1], Lemmas 4, 5, $\left.9(i v)\right)$. So the operator

$$
\left(\Delta_{\nu}^{\bullet}-\lambda_{0}\right)^{-1}=R_{\lambda_{0}}^{m}+\left(\Delta_{\nu}^{\bullet}-\lambda_{0}\right)^{-1}\left(I-\left(\Delta_{\nu}^{\bullet}-\lambda_{0}\right) R_{\lambda_{0}}^{m}\right)
$$

is compact in $\left(D R^{\bullet}(M)\right)_{2}$. Since $G_{\lambda_{0}}(\nu)$ is a compact operator for $\lambda_{0} \in \Lambda_{\varepsilon},\left|\lambda_{0}\right|>>1$, and since $\Delta_{\nu}^{\bullet}$ is a closed operator in $\left(D R^{\bullet}(M)\right)_{2}$, it follows that $\Delta_{\nu}^{\bullet}$ is an operator in $\left(D R^{\bullet}(M)\right)_{2}$ with compact resolvent. So (according to [Ka], Ch. 3, Theorem 6.29) its spectrum $\operatorname{Spec}\left(\Delta_{\nu}^{\bullet}\right)$ consists of isolated eigenvalues with finite multiplicities (i.e., $\operatorname{Spec}\left(\Delta_{\nu}^{\bullet}\right)$ is discrete) and the operator $G_{\lambda}^{\bullet}(\nu)$ is compact in $\left(D R^{\bullet}(M)\right)_{2}$ for $\lambda \in$ $\mathbb{C} \backslash \operatorname{Spec}\left(\Delta_{\nu}^{\bullet}\right)$. The operator $\Delta_{\nu}^{\bullet}$ is nonnegative. Hence $\operatorname{Spec}\left(\Delta_{\nu}^{\bullet}\right) \subset \mathbb{R}_{+} \cup 0$.

If $\lambda_{0} \notin \operatorname{Spec}\left(\Delta_{\nu}^{\bullet}\right)$ then $\operatorname{Ker}\left(\Delta_{\nu}^{\bullet}-\lambda_{0}\right)=0$ and $\operatorname{Im}\left(\Delta_{\nu}^{\bullet}-\lambda_{0}\right)=(D R \cdot(M))_{2}$. Hence ind $\left(\Delta_{\nu}^{\bullet}-\lambda_{0}\right)$ is equal to 0 (as the index of the operator from $\left(\operatorname{Dom}\left(\Delta_{\nu}^{\bullet}\right),\|\cdot\|_{\text {graph }}^{2}\right)$ into $\left.\left(\left(D R^{\bullet}(M)\right)_{2},\|\cdot\|_{2}^{2}\right)\right)$. The operator $\left(\lambda_{0}-\lambda\right)$ id from $\operatorname{Dom}\left(\Delta_{\nu}^{\bullet}\right)$ into $\left(D R^{\bullet}(M)\right)_{2}$ is compact (since $G_{\lambda_{0}}^{\bullet}(\nu)$ is a compact operator in $\left(D R^{\bullet}(M)\right)_{2}$ and since it is a topological isomorphism $\left.G_{\lambda_{0}}(\nu):\left(D R^{\bullet}(M)\right)_{2} \underset{\sim}{\sim} \operatorname{Dom}\left(\Delta_{\nu}^{\bullet}\right)\right)$. So ind $\left(\Delta_{\nu}^{\bullet}-\lambda\right)=0$ for an arbitrary $\lambda \in \mathbb{C}$ (according to $[\mathrm{Ka}]$, Ch. 4, Theorem 5.26, Remarks 1.12, 1.4).

8. The operator $\left(\Delta_{\nu}^{\bullet}\right)^{-s}$ for Re $s>0$ is defined by the integral

$$
\frac{i}{2 \pi} \int_{\Gamma} \lambda^{-s}\left(\Delta_{\nu}^{\bullet}-\lambda\right)^{-1} d \lambda=: T_{-s}(\nu)
$$


where the contour $\Gamma$ is

$$
\left\{\lambda=r e^{i \pi}, \infty>r>\varepsilon\right\} \cup\left\{\lambda=\varepsilon e^{i \varphi}, \pi>\varphi>-\pi\right\} \cup\left\{\lambda=r e^{-i \pi}, \varepsilon<r<\infty\right\} .
$$

Here the number $\varepsilon>0$ is such that $\operatorname{Spec}\left(\Delta_{\nu}^{\bullet}\right) \cap(0, \varepsilon]=\emptyset$. The integral (3.18) is absolutely convergent (with respect to the operator norm $\|\cdot\|_{2}$ in $\left.\left(D R^{\bullet}(M)\right)_{2}\right)$ because the estimate $\left\|\left(\Delta_{\nu}^{\bullet}-\lambda\right)^{-1}\right\|_{2} \leq C|\lambda|^{-1}$ is satisfied as $\lambda \rightarrow-\infty$ for $\lambda \in \mathbb{R}_{-}$. So $T_{-s}(\nu)$ is a bounded operator in $(D R \bullet(M))_{2}$ for Re $s<0$.

For $-k \geq \operatorname{Re} s>-(k+1), k \in \mathbb{Z}_{+}$, the operator $T_{-s}$ is defined as $\Delta_{\nu}^{k+1} T_{-(s+k+1)}$. Its domain is $\operatorname{Dom}\left(T_{-s}\right)=\left\{\omega \in\left(D R^{\bullet}(M)\right)_{2}, T_{-(s+k+1)} \omega \in \operatorname{Dom}\left(\left(\Delta_{\nu}^{\bullet}\right)^{k+1}\right)\right\}$, where

$$
\operatorname{Dom}\left(\left(\Delta_{\nu}^{\bullet}\right)^{k+1}\right):=\left\{\omega \in \operatorname{Dom}\left(\Delta_{\nu}^{\bullet}\right), \Delta_{\nu}^{\bullet} \omega \in \operatorname{Dom}\left(\Delta_{\nu}^{\bullet}\right), \ldots,\left(\Delta_{\nu}^{\bullet}\right)^{k} \omega \in \operatorname{Dom}\left(\Delta_{\nu}^{\bullet}\right)\right\}
$$

The restriction $T_{-s}^{0}$ of $T_{s}$ to the orthogonal complement $L_{0}$ of $\operatorname{Ker}\left(\Delta_{\nu}^{\bullet}\right)$ in $\left(D R^{\bullet}(M)\right)_{2}$ is defined on $\operatorname{Dom}\left(T_{-s}^{0}\right):=D\left(T_{-s}\right) \cap L_{0}, T_{-s}^{0}:=\left.T_{-s}\right|_{L_{0}}$. Then $T_{-s}$ is the direct $\operatorname{sum}^{56}$ of $T_{s}^{0}$ and of the zero operator on $\operatorname{Ker}\left(\Delta_{\nu}^{\bullet}\right)$. Theorem 1 in [Se2] claims that the family $T_{-s}^{0}$ of operators in the Hilbert space $L_{0}$ for Re $s_{2}>0$ satisfies the equation $T_{-s_{1}}^{0} T_{-s_{2}}^{0}=T_{-\left(s_{1}+s_{2}\right)}^{0}$ and that the same is true for $-s_{1} \in \mathbb{Z}_{+}$and for each $s_{2}$. This theorem claims also that

$$
T_{0}^{0}=\mathrm{id} \text { on } L_{0}, \quad T_{-l}^{0}=\left(\left.\left(\Delta_{\nu}^{\bullet}\right)^{-1}\right|_{L_{0}}\right)^{l}, \text { for } l \in \mathbb{Z}_{+}, \text {and } T_{1}^{0}=\left.\Delta_{\nu}^{\bullet}\right|_{L_{0}}
$$

(the domain of $T_{1}^{0}$ is $\left.\operatorname{Dom}\left(\Delta_{\nu}^{\bullet}\right) \cap L_{0}\right)$ and that $T_{-s}^{0}$ for Re $s>0$ is a holomorphic function $^{57}$ with its values in a Banach space $B\left(L_{0}\right)$ of bounded operators in $L_{0}$ where the Banach norm is the operator norm as the norm.

9 . For Re $s>n / 2$ the kernel of $T_{-s}(\nu)$ is continuous on $\bar{M}_{j_{1}} \times \bar{M}_{j_{2}}$ and analytic in $s\left([\mathrm{Se} 2]\right.$, Theorem 2(i)). For $\operatorname{Re} s>n / 2$ the zeta-function $\zeta_{\nu, \bullet}(s)$ is equal to the sum of integrals over the diagonals $\bar{M}_{j} \hookrightarrow \bar{M}_{j} \times \bar{M}_{j}(j=1,2)$ of the densities defined by the restrictions to these diagonals of the kernel $T_{-s}(\nu)$, according to Proposition 3.9 below. So $\zeta_{\nu, \bullet}(s)$ is holomorphic for $\operatorname{Re} s>n / 2$.

The operator $G_{\lambda}^{\bullet}(\nu)-P_{\lambda}^{m}$ for $m \geq n$ (where $P_{\lambda}^{m}$ is the parametrix (3.3)) is a bounded in $\left(D R^{\bullet}(M)\right)_{2}$ operator with a continuous on $\bar{M}_{j_{1}} \times \bar{M}_{j_{2}}$ kernel $\left(r_{\lambda}^{m}\right)_{x_{1}, x_{2}}$ which is

$$
\left(r_{\lambda}^{m}\right)_{x_{1}, x_{2}}=O\left(\left(1+|\lambda|^{1 / 2}\right)^{-(2+m)+n}\right)
$$

\footnotetext{
${ }^{56}$ If $v \in L_{0}$ and $\operatorname{Re} s>0$ then we have $T_{-s} v \in L_{0}$ since for $h \in \operatorname{Ker}\left(\Delta_{\nu}^{\bullet}\right)$ and $\lambda \in \Gamma$ it holds

$$
0=(v, h)=\left(\left(\Delta_{\nu}^{\bullet}-\lambda\right) G_{\lambda}^{\bullet}(\nu) v, h\right)=-\lambda\left(G_{\lambda}^{\bullet}(\nu) v, h\right)
$$

and since the integral (3.18) is absolutely convergent. For $h \in \operatorname{Ker}\left(\Delta_{\nu}^{\bullet}\right)$ and for $\operatorname{Re} s>0$ we have $T_{-s} h=0$ because for such $s$ the integral $\int_{\Gamma} \lambda^{-s-1} d \lambda$ is absolutely convergent and is equal to zero. Since $T_{-s} h=\left(\Delta_{\nu}^{\bullet}\right)^{k+1} T_{-(s+k+1)} h=0$ for $-k \geq \operatorname{Re} s>-(k+1)$, we get $T_{-s} h \equiv 0$ for all $s$.

${ }^{57} \mathrm{~A}$ function with the values in a Banach space is holomorphic in a strong sense if it is weakly holomorphic ([Ka], Ch. $3, \S 1$, Theorem 1.37, p. 139)
} 
as $|\lambda| \rightarrow+\infty, \lambda \in \Lambda_{\varepsilon}$ ([Se1], Theorem 1, or also the assertions 5, 1, 2, 7 above). So the operator

$$
\frac{i}{2 \pi} \int_{\Gamma} \lambda^{-s}\left(G_{\lambda}^{\bullet}(\nu)-P_{\lambda}^{m}\right) d \lambda
$$

for Re $s>(n-m) / 2$ is of trace class and its kernel is continuous on $\bar{M}_{j_{1}} \times \bar{M}_{j_{2}}$ and analytic in $s$.

The trace of the operator (3.20) is holomorphic in $s$ for $\operatorname{Re} s>(n-m) / 2$. Let us denote by $K_{x, y}^{\text {int }}(s)$ the kernel of the operator $(i / 2 \pi) \int_{\Gamma} \lambda^{-s} P_{\lambda, \text { int }}^{m} d \lambda$ (where $P_{\lambda, \text { int }}^{m}:=$ $\sum \psi_{j} P_{\lambda, U_{j}, \text { int }}^{m} \varphi_{j}$ is a term of (3.3) and $P_{\lambda, U_{j}, \text { int }}^{m}$ is a PDO with symbol $\theta s_{(m)}$, defined by (3.4)). This kernel is continuous on $\bar{M}_{j_{1}} \times \bar{M}_{j_{2}}$ for Re $s>n / 2$. Off the diagonals $\bar{M}_{j} \hookrightarrow \bar{M}_{j} \times \bar{M}_{j}$ it extends to a kernel which is an entire function of $s \in \mathbb{C}$ equal to zero for $(-s) \in \mathbb{Z}_{+} \cup 0$. The density on $\cup_{j} \bar{M}_{j}$ defined by the restriction of this kernel to the diagonals also can be continued to a meromorphic in $s \in \mathbb{C}$ density. This density has at most simple poles at $s_{j}=(n-j) / 2$ for $\left(-s_{j}\right) \notin \mathbb{Z}_{+} \cup 0,0 \leq j \leq m$, and it is regular at $s_{j}$ for $\left(-s_{j}\right) \in \mathbb{Z}_{+} \cup 0$.

The residue at $s=s_{j}$ is completely defined by the component $a_{-2-j}(x, \xi, \lambda)$ of the symbol $s\left(\left(\Delta^{\bullet}-\lambda\right)^{-1}\right)([\mathrm{Se} 2]$, Lemma 1 or [Sh], Theorem 12.1). These components are given by (3.4). The value of this density at $s=s_{j}$ for $\left(-s_{j}\right) \in \mathbb{Z}_{+} \cup 0$ is completely defined by $a_{-2-j}$ (by the formulas (11), (12) in [Se2] with changing of the sign in (11) to the opposite one). Here, $j=n+2 m, m \in \mathbb{Z}_{+} \cup 0$.

The kernel $K_{x, y}^{\partial}(s)$ of the operator ${ }^{58}(i / 2 \pi) \int_{\Gamma} \lambda^{-s} \mathcal{D}_{m, \lambda} d \lambda$ for Re $s>n / 2$ is continuous on $\bar{M}_{j_{1}} \times \bar{M}_{j_{2}}$ and analytic in $s$ ([Se2], Lemma 4). Let $(x, y)$ be off the diagonals or let either $x$ or $y$ be not from $\cup_{j} \partial \bar{M}_{j} \supset N$. Then $K_{x, y}^{\partial}(s)$ is an entire function of $s \in \mathbb{C}$ and it is equal to zero at $s$ for $(-s) \in \mathbb{Z}_{+} \cup 0$ ([Se2], Lemma 4). For Re $s>n / 2$ the densities defined by the restriction $K_{x, x}^{\partial}(s)$ of $K_{x, y}^{\partial}(s)$ to the diagonals $\bar{M}_{j}$ are integrable over the fibers of the natural projections $p_{1}:[0,1] \times N \rightarrow N$ and $p_{2}:[-1,0] \times N \rightarrow N$. These integrals are densities on $N$. They can be continued ${ }^{59}$ to meromorphic on $s \in \mathbb{C}$ densities (on $N$ ) with at most simple poles at $s_{j}=(n-j) / 2$, $1 \leq j \leq m$, such that $\left(-s_{j}\right) \notin \mathbb{Z}_{+} \cup 0$. Its residues at $s_{j}$ for $1 \leq j \leq m+1$ are completely defined by a term $d_{-2-j+1}$ in $d$ ([Se2], Theorem $2(i v)$, formula (II)). The values of these densities at $s_{j}$ for $\left(-s_{j}\right) \in \mathbb{Z}_{+} \cup 0$ (where $n \leq j \leq m+1$ ) are also completely defined by $d_{-2-j+1}$ ([Se2], Lemmas 2,3 , 4, Theorem $2(i v)$, formula (II')

\footnotetext{
${ }^{58}$ Here $\mathcal{D}_{m, \lambda}=\sum \psi_{j} \mathcal{D}_{m, U}(\lambda, \nu) \psi_{j}$. The operator $\mathcal{D}_{m, U}$ from (3.13) is defined for $U \cap N \neq \emptyset$ by (3.12), (3.9), and (3.10).

${ }^{59}$ Let $\mathcal{L} \mathcal{D}_{m, U}(\lambda, \nu)$ be an operator acting on $\mathcal{L} \omega$ as $\mathcal{L}\left(\mathcal{D}_{m, U}(\lambda, \nu) \omega\right.$ ) (for any $\omega \in D R_{c}^{\bullet}\left(\mathbb{R}^{n}\right)$ such that $\operatorname{supp} \omega \cap \mathbb{R}^{n-1}=\emptyset$, where $\mathbb{R}^{n-1}$ are local coordinates on $\left.N\right)$. All the assertions about the kernels, analogous to $K_{x, y}^{\partial}(s)$ in the case of $\mathcal{L D}_{m, U}(\lambda, \nu)$, and about the corresponding densities in this case, are proved in [Se2]. Thus the transformation (3.7) provides us with all the assertions about the kernel $K_{x, y}^{\partial}(s)$ (and about the corresponding densities) connected with $\mathcal{D}_{U, \lambda}(\nu)$.
} 
with changing of the sign to the opposite one).

10. The kernel of the operator (3.20) is the difference of the kernels

$$
\left(T_{-s}\right)_{x, y}-\left(K_{x, y}^{i n t}(s)-K_{x, y}^{\partial}(s)\right) .
$$

For Re $s>(n-m) / 2$ it is holomorphic in $s$ and continuous on $\bar{M}_{j_{1}} \times \bar{M}_{j_{2}}$. The term $\left(K^{i n t}-K^{\partial}\right)_{x, y}(s)$ is equal to zero for $x \neq y$ and $(-s) \in \mathbb{Z}_{+} \cup 0$. The term $\left(T_{-s}\right)_{x, y}$ is equal to zero for $x \neq y$ and $(-s) \in \mathbb{Z}_{+}$(according to the assertions of 8 above, since $\Delta_{\nu}^{\bullet}$ is a differential operator). We have $\left(T_{0}\right)_{x, y}=-\mathcal{H}_{x, y}^{\bullet}$, where $\mathcal{H}^{\bullet}$ is the kernel of the orthogonal projection operator in $\left(D R^{\bullet}(M)\right)_{2}$ onto $\operatorname{Ker}\left(\Delta_{\nu}^{\bullet}\right)$ (the assertion 8 ). The properties of $\zeta_{\nu, \bullet}(s)^{60}$ formulated in Theorem 3.1, follow from the assertions of 9 and 10. The theorem is proved.

Remark 3.1. The kernel (3.21) of the operator (3.20) is holomorphic in $s$ and continuous in $(x, y) \in \bar{M}_{j_{1}} \times \bar{M}_{j_{2}}$ for $\operatorname{Re} s>(n-m) / 2$. It is equal to zero for $x \neq y$ at $s=-k, k \in \mathbb{Z}_{+}$, and to $-\mathcal{H}_{x, y}^{\bullet}(\nu)$ at $s=0$. So the analytic continuations of the densities on $\bar{M}_{j}$ and on $N$ defined by the kernels $\left(T_{-s}\right)_{x, y}$ and $\left(K^{\text {int }}(s)-K^{\partial}(s)\right)_{x, y}$ have the same residues at $s=s_{j}, 0 \leq j \leq m,\left(-s_{j}\right) \notin \mathbb{Z}_{+} \cup 0$, and the same values at $s=s_{j},\left(-s_{j}\right) \in \mathbb{Z}_{+}, n+2 \leq j \leq m$. They differ at $s=0$ (i.e., for $j=n$ ) by the densities on $\bar{M}_{j}$, defined by $-\mathcal{H}_{x, x}^{\bullet}(\nu)$. Hence the densities on $\bar{M}_{j}$ and on $N$, corresponding to the residues and to the values at $s=s_{j}, 0 \leq j \leq m-1$, of $\left(K^{i n t}(s)-K^{\partial}(s)\right)_{x, x}$ are the same for all the parametrixes $P_{\lambda}^{m}$ defined by (3.3) (with different covers $\left\{U_{j}\right\}$, partitions of unity $\left\{\varphi_{j}\right\}$ subordinate to $\left\{U_{j}\right\}$, and $\left\{\psi_{j}\right\}$ ).

And back, the values and the residues of the analytic continuation for the integral $\int_{\bar{M}_{j}} \operatorname{tr}\left(i_{j}^{*}\left(T_{-s}\right)\right)+\delta_{s, 0} \int_{\bar{M}_{j}} \operatorname{tr}\left(i_{j}^{*} \mathcal{H}^{\bullet}(\nu)\right)$ at $s_{j}, 0 \leq j \leq m$, are defined by an arbitrary parametrix $P_{\lambda}^{m}$.

Proof of Proposition 3.1. Let $m \geq n:=\operatorname{dim} M, m \in \mathbb{Z}_{+}$, and $\lambda \in \Lambda_{\varepsilon}$. Then the parametrix $R_{\lambda}^{m}$ for $G_{\lambda}^{\bullet}(\nu)^{61}$ (defined by (3.17)) is a bounded operator ${ }^{62}$

${ }^{60}$ The values of $\zeta_{\nu, \bullet}(s)$ at $(-s) \in \mathbb{Z}_{+}$and the residues of $\zeta_{\nu, \bullet}$ at $s=s_{j}$ can be also expressed in terms of noncommutative residues ([Wo] or $[\mathrm{Kas}]$ ). The density on $M$ whose integral over $M$ is equal to a volume term in $\operatorname{Res}_{s=s_{j}} \zeta_{\nu, \bullet}(s)$ can be written as $2^{-1}$ res $\left(x, \Delta_{\theta=\pi}^{-s_{j}}\right)$. Here res is a noncommutative residue for the symbol of PDO $\Delta_{\theta=\pi}^{-s_{j}}$. This symbol is defined with the help of the symbol $\sum a_{-2-j}(x, \xi, \lambda)$ of $\left(\Delta^{\bullet}-\lambda\right)^{-1}$ ([Sh], 11.2). The boundary term in $\operatorname{Res}_{s=s_{j}} \zeta_{\nu, \bullet}(s)$ for $\left(-s_{j}\right) \notin \mathbb{Z}_{+} \cup 0$ is expressed similarly.

${ }^{61}$ The statement that $G_{\lambda}^{\bullet}(\nu):\left(D R^{\bullet}(M)\right)_{2} \rightarrow \operatorname{Dom}\left(\Delta_{\nu}^{\bullet}\right)$ is an isomorphism for $\lambda \notin \operatorname{Spec}\left(\Delta_{\nu}^{\bullet}\right)$ is proved in Theorem 3.1 .

${ }^{62}$ The terms $P_{\lambda, i n t}^{(m)}, \psi_{j} \mathcal{D}_{m} \varphi_{j}$, and $q_{\nu} B_{\nu} P_{\lambda}^{m}$ in $R_{\lambda}^{m}$ are bounded operators with the same estimate of their norms in $\left(D R^{\bullet}(M)\right)_{2}$ for $\lambda \in \Lambda_{\varepsilon}$ (the proof of Theorem 3.1). For the sake of brevity the proof of Proposition 3.1 is given in the case of $\partial M=\emptyset$. 
in $\left(D R^{\bullet}(M)\right)_{2}$ with its norm estimated by $O\left(\left(1+|\lambda|^{1 / 2}\right)^{-2}\right)$ for $\lambda \in \Lambda_{\varepsilon}$. It holds that $R_{\lambda}:\left(D R^{\bullet}(M)\right)_{2} \rightarrow \operatorname{Dom}\left(\Delta_{\nu}^{\bullet}\right)$. For a linear differential operator $F$ of order $d=d(F) \leq 2$ the operator $F R_{\lambda}$ is defined on smooth forms $\omega \in D R_{c}^{\bullet}(M \backslash N)$ and its closure in $\left(D R^{\bullet}(M)\right)_{2}$ is a bounded operator in $\left(D R^{\bullet}(M)\right)_{2}$ with its norm estimated by $O\left(\left(1+|\lambda|^{1 / 2}\right)^{d-2}\right)$ for $\lambda \in \Lambda_{\varepsilon}$. (All these estimates are uniform with respect to $\nu \in \mathbb{R}^{2} \backslash(0,0)$.) The only terms of $R_{\lambda}^{m}$ depending on $\nu$ are the terms $\mathcal{D}-q_{\nu} B_{\nu} P_{\lambda}^{m}(\nu)$, where ${ }^{63} \mathcal{D}=\mathcal{D}(\nu):=-\sum \psi_{j} \mathcal{D}_{m, U_{j}} \varphi_{j}$ has the kernel with support in the neighborhood $I \times N$ of the interior boundary $N \hookrightarrow M{ }^{64}$ (The parametrix $P_{\lambda}^{m}(\nu):=P_{\lambda, \text { int }}^{m}-\mathcal{D}(\nu)$ is defined by (3.13)). We need the following assertion now.

Proposition 3.3. The operators $\mathcal{D}(\nu)$ and $q_{\nu} B_{\nu} P_{\lambda}^{m}(\nu)$ depend smoothly on $\nu \in$ $\mathbb{R}^{2} \backslash(0,0)$ as bounded operators in $\left(D R^{\bullet}(M)\right)_{2}$. For a $C^{\infty}{ }_{-m a p} \nu=\varphi(\gamma):[-a, a] \rightarrow$ $\mathbb{R}^{2} \backslash(0,0)$ the operator $\partial_{\gamma} \mathcal{D}(\nu)$ is a bounded operator in $\left(D R^{\bullet}(M)\right)_{2}$ whose norm is uniformly with respect to $\gamma$ estimated by $O\left(\left(1+|\lambda|^{1 / 2}\right)^{-2}\right)$ for $\lambda \in \Lambda_{\varepsilon}$. Let $F$ be a linear differential operator of order $d=d(F) \leq 2$ from $D R^{\bullet}\left(\bar{M}_{j}\right)$ into $D R^{\bullet+k}\left(\bar{M}_{j}\right), k \in \mathbb{Z}$. Then $F \mathcal{D}(\nu), F \partial_{\gamma} \mathcal{D}(\nu)$ are bounded operators from $\left(D R^{\bullet}(M)\right)_{2}$ into $\left(D R^{\bullet+k}(M)\right)_{2}$ whose norms are estimated by $O\left(\left(1+|\lambda|^{1 / 2}\right)^{d(F)-2}\right)$ for $\lambda \in \Lambda_{\varepsilon}$. The operator $\partial_{\gamma}\left(q_{\nu} B_{\nu} P_{\lambda}^{m}(\nu)\right)$ is uniformly with respect to $\gamma$ estimated by $O\left(\left(1+|\lambda|^{1 / 2}\right)^{-1}\right)$ for $\lambda \in \Lambda_{\varepsilon}$.

Proof. The kernel of the operator $\mathcal{L} \psi_{j} \mathcal{D}_{m, U_{j}} \varphi_{j} \mathcal{L}^{-1}$ (where $\mathcal{L}=\mathcal{L}(\nu)$ and $\mathcal{L}^{-1}$ are defined by $(3.5)$ and $(3.7))$ has a support in $\left(\left(U_{j} \cap N\right) \times[0,1]\right)^{2}$. The operator $\mathcal{D}_{m, U_{j}}$ is defined in $\left(U_{j} \cap N\right) \times \mathbb{R}_{+}$by (3.11) and (3.10). The right sides of the boundary conditions (3.10) depend on $\nu$ only by their dependence on $L(\nu)$ (where $L$ is the matrix defined by (3.7)). Since $g_{M}$ is a direct product metric near $N$, a mirror symmetry (relative to $N$ ) acts as the identity operator on the symbol $\sum a_{-2-j}(x, \xi)$ of the Laplacian $\Delta^{\bullet}$ on $M$ for $x=\left(t, x^{\prime}\right)$ from the neighborhood $I \times N$ of $N$. The symbol $\sum a_{-2-j}\left(t, x^{\prime}, \tau, \xi^{\prime}, \lambda\right)$ is independent of $t$ for $t \in I$.

So the symbol $\mathcal{L} \sum a_{-2-j}$ (for $t \in I$ ) is expressed as $L a L^{-1}$, where $L$ and $L^{-1}$ act on the components of a matrix valued functions $a_{-2-j}$ in the coordinates $\omega_{j, c}$ and $\omega_{j,(1, f)}$ as follows (according to (3.5)):

$$
\begin{aligned}
& (L a)_{1, c ; *}=|\nu|^{-1}(\alpha-\beta)(a)_{c ; *}, \quad(L a)_{1,(1, f) ; *}=|\nu|^{-1}(-\beta+\alpha)(a)_{(1, f) ; *}, \\
& (L a)_{2, c ; *}=|\nu|^{-1}(\beta+\alpha)(a)_{c ; *}, \quad(L a)_{2,(1, f) ; *}=|\nu|^{-1}(\alpha+\beta)(a)_{(1, f) ; *} .
\end{aligned}
$$

\footnotetext{
${ }^{63} \mathcal{D}_{m}=\mathcal{D}_{m, U_{j}}$ is defined by (3.11), (3.10), and (3.12).

${ }^{64}$ In the case $\partial M \neq \emptyset$ the terms connected with the Dirichlet and the Neumann boundary conditions are added to $\mathcal{D}(\nu)$. Then the corresponding kernel has its support in a neighborhood of $\partial M$ in $M$.
} 
The boundary conditions (3.10) (according to (3.22)) depend on $\nu$ only by the matrix transformation whose coefficients are independent of $\left(t, x^{\prime}\right)$ and smooth in $\nu$. This transformation acts separately on each homogeneous component $a_{-2-j}$. The right sides in (3.22) are nonsingular in $\left(x^{\prime}, \tau, \xi^{\prime}, \lambda\right)$ for $b_{2}\left(x^{\prime}, \tau, \xi^{\prime}\right)-\lambda \neq 0$ (where $b_{2}$ is the principal symbol of the Laplacian on $M$ for $\left.\left(t, x^{\prime}\right) \in I \times N\right)$. Hence Lemma 2 in [Se1] holds also for the symbol $\partial_{\gamma}\left(\mathcal{L}(\nu) \sum d_{-2-j}\right)$. Thus the desired estimates for the norm of $\partial_{\gamma} \mathcal{D}(\nu)$ in $\left(D R^{\bullet}(M)\right)_{2}$ and for the norms of $F \mathcal{D}(\nu)$ and of $F \partial_{\gamma} \mathcal{D}(\nu)$ are consequences of [Se1], Lemma 7 .

The operators $q_{j, \nu}(1 \leq j \leq 4)$ from (3.14) and (3.16) (for $\varphi(t)$ even on $t$ ) can be defined such that ${ }^{65}$

$$
\mathcal{L} q_{\nu} B_{\nu} P_{\lambda}^{m}(\nu) f(t) \mathcal{L}^{-1}=\sum q_{j} B_{j} \mathcal{L} P_{\lambda}^{m}(\nu) f(t) \mathcal{L}^{-1},
$$

where $f \in C_{0}^{\infty}(I), f(t) \equiv 1$ for $t \in[0,1 / 2]$ and $f(t) \equiv 0$ for $t>3 / 4$. The operators $B_{j}$ and $q_{j}$ are independent of $\nu \in \mathbb{R}^{2} \backslash(0,0)$ and correspond to $B_{j, \nu}$ and $q_{j, \nu}$ from (3.15), (3.16). (Here $B_{1}, B_{2}, B_{3}, B_{4}$ are the operators (3.6) acting respectively on $\left.v_{1, c}, w_{2,(1, f)}, w_{1,(1, f)}, w_{2, c}.\right)$ These operators are such that $B_{i} q_{j}=\delta_{i j}$ id.

The operator $\sum q_{j} B_{j} \mathcal{L}\left(\partial_{\gamma} P_{\lambda}^{m}(\nu)\right) f \mathcal{L}^{-1}$ is equal to $\sum q_{j} B_{j} \mathcal{L}\left(-\partial_{\gamma} \mathcal{D}(\nu) f\right) \mathcal{L}$ (since $P_{\lambda, \text { int }}^{m}$ is independent of $\left.\nu\right)$. The operator $B_{j} \mathcal{L}\left(\partial_{\gamma} \mathcal{D}(\nu)\right) \mathcal{L}^{-1}$ is defined on smooth forms $\omega \in D R_{c}^{\bullet}((0,1) \times N)$ and its $L_{2}$-norm is estimated by $O\left(\left(1+|\lambda|^{1 / 2}\right)^{-1}\right)$ for $\lambda \in \Lambda_{\varepsilon}$ ([Se1], Lemma 7). The operators $\partial_{\gamma}\left(q_{\nu} B_{\nu}\right) P_{\lambda, \text { int }}^{m}$ are defined on smooth forms $\omega \in D R_{c}^{\bullet}(M \backslash N)$ and their operators $L_{2}$-norms are estimated by $O\left(\left(1+|\lambda|^{1 / 2}\right)^{-1}\right)$ for $\lambda \in \Lambda_{\varepsilon}$ uniformly with respect to $\nu$ (according to [Se1], Lemma 7). The proposition is proved.

Let $\lambda \in \Lambda_{\varepsilon}$ and $|\lambda|$ be large enough. Then the Green function $G_{\lambda}^{\bullet}(\nu)$ can be represented by the series

$$
G_{\lambda}^{\bullet}(\nu)=R_{\lambda}^{m} \sum_{i=0}^{\infty}\left(L_{\lambda}^{m}\right)^{i},
$$

where $\left(L_{\lambda}^{m}\right):=\mathrm{id}-\left(\Delta_{\nu}^{\bullet}-\lambda\right) R_{\lambda}^{m}$ is a bounded operator in $\left(D R^{\bullet}(M)\right)_{2}$ for $\lambda \in$ $\Lambda_{\varepsilon}$. The norm of $L_{\lambda}^{m}$ in $\left(D R^{\bullet}(M)\right)_{2}$ is $O\left(\left(1+|\lambda|^{1 / 2}\right)^{n-m+2}\right)$ (where $\left.n:=\operatorname{dim} M\right)$ because the norm of $\left(\mathrm{id}-\left(\Delta^{\bullet}-\lambda\right) P_{\lambda}^{m}\right)$ is $O\left(\left(1+|\lambda|^{1 / 2}\right)^{n-m}\right)$ and the norm of $\left(\Delta^{\bullet}-\lambda\right) q_{\nu} B_{\nu} P_{\lambda}^{m}$ is $O\left(\left(1+|\lambda|^{1 / 2}\right)^{n-m+2}\right)$ (according to the proof of Theorem 3.1). Hence if $m>n+2$ and if $\lambda \in \Lambda_{\varepsilon}$ with $|\lambda|$ large enough then the series (3.23) is

\footnotetext{
${ }^{65}$ The operator $B_{\nu} P_{\lambda}^{m}(\nu)$ has a continuous on $\bar{M}_{j_{1}} \times \bar{M}_{j_{2}}$ kernel which is estimated uniformly with respect to $\left(x_{1}, x_{2}\right) \in\left(N \cap \bar{M}_{j_{1}}\right) \times \bar{M}_{j_{2}}$ and to $\nu \in \mathbb{R}^{2} \backslash(0,0)$ by $O\left(\left(1+|\lambda|^{1 / 2}\right)^{n-m}\right)$ for $\lambda \in \Lambda_{\varepsilon}$ ([Se1], Lemma 6). Such an estimate holds also for the kernel of $q_{\nu} B_{\nu} P_{\lambda}^{m}(\nu)$.
} 
convergent with respect to the operator norm in $\left(D R^{\bullet}(M)\right)_{2}$. The operator $L_{\lambda}^{m}$ depends smoothly on $\nu \in \mathbb{R}^{2} \backslash(0,0)$. The norm of $\partial_{\gamma} L_{\lambda}^{m}$ is estimated by $O\left(1+|\lambda|^{1 / 2}\right)$ (according to Proposition 3.3). Let $\nu:[-a, a] \rightarrow \mathbb{R}^{2} \backslash(0,0)$ be a smooth map. Then the series for $\partial_{\gamma} G_{\lambda}^{\bullet}(\nu)$ is convergent (in the operator norm) if $\lambda \in \Lambda_{\varepsilon}$ and if $|\lambda|$ is large enough. Hence for such $\lambda$ the resolvent $G_{\lambda}^{\bullet}(\nu):=\left(\Delta_{\nu}^{\bullet}-\lambda\right)^{-1}$ depends smoothly on $\gamma$. So the family $G_{\lambda}^{\bullet}(\nu)$ of bounded operators in $\left(D R^{\bullet}(M)\right)_{2}$ is smooth in $(\nu, \lambda)$ for such $\lambda$. Their operator norms are estimated by $O\left(|\lambda|^{-1}\right)$ uniformly with respect to $\nu \in \mathbb{R}^{2} \backslash(0,0)$. Let $F$ be a linear differential operator of degree $d(F) \leq 2$. Then the operators $F G_{\lambda}^{\bullet}(\nu)$ for such $\lambda$ are bounded in $\left(D R^{\bullet}(M)\right)_{2}$ with their operator norms estimated by $O\left(|\lambda|^{(d-2) / 2}\right)$ uniformly with respect to $\nu$. These operators depend smoothly on $\nu$ for such $\lambda$ and we have

$$
\partial_{\gamma} F G_{\lambda}^{\bullet}(\nu)=F \partial_{\gamma} G_{\lambda}^{\bullet}(\nu)
$$

Hence for a given $\nu_{0} \in \mathbb{R}^{2} \backslash(0,0)$ there exists $\lambda_{1} \in \Lambda_{\varepsilon}$ such that $G_{\lambda_{1}}^{\bullet}(\nu)$ depends smoothly on $\nu$ for $\nu$ sufficiently close to $\nu_{0}$. For $\lambda \in \mathbb{C} \backslash \operatorname{Spec}\left(\Delta_{\nu_{0}}^{\bullet}\right)$ the resolvent $G_{\lambda}^{\bullet}\left(\nu_{0}\right)$ can be represented as follows:

$$
G_{\lambda}^{\bullet}\left(\nu_{0}\right)=-\left(\lambda-\lambda_{1}\right)^{-1}-\left(\lambda-\lambda_{1}\right)^{-2} R\left(\left(\lambda-\lambda_{1}\right)^{-1}, G_{\lambda_{1}}^{\bullet}\left(\nu_{0}\right)\right)
$$

where $R\left(\eta, G_{\lambda_{1}}^{\bullet}\left(\nu_{0}\right)\right):=\left(G_{\lambda_{1}}^{\bullet}\left(\nu_{0}\right)-\eta\right)^{-1}$ is the resolvent of a bounded operator $G_{\lambda_{1}}^{\bullet}\left(\nu_{0}\right)$ in $\left(D R^{\bullet}(M)\right)_{2}([\mathrm{Ka}]$, Ch. IV, (3.6), Ch. III, (6.18)). The bounded operator $R(\eta, B)$ is an analytic function of a bounded operator $B$ and of $\eta$ for $\eta \notin$ Spec $B$ (i.e., near $\left(\eta_{0}, B_{0}\right), \eta_{0} \notin \operatorname{Spec} B_{0}$, it is locally defined by a convergent double power series in $\left(\eta-\eta_{0}\right)$ and $\left.\left(B-B_{0}\right)\right)$. The operator $G_{\lambda_{1}}^{\bullet}(\nu)$ depends smoothly on $\nu$ for $\nu$ sufficiently close to $\nu_{0}$. Then it follows from (3.25) that $G_{\lambda}^{\bullet}(\nu)$ depends smoothly on $\nu$ for $\lambda \in \mathbb{C} \backslash \operatorname{Spec}\left(\Delta_{\nu}^{\bullet}\right)$ and for $\nu$ sufficiently close to $\nu_{0}$.

Let $F: \oplus_{j} D R^{\bullet}\left(\bar{M}_{j}\right) \rightarrow \oplus_{j} D R^{\bullet+k}\left(\bar{M}_{j}\right), k \in \mathbb{Z}$, be a linear differential operator of degree $d(F) \leq 2$. Then for $\lambda \in \Lambda_{\varepsilon}$ and $|\lambda|$ large enough the operators $F G_{\lambda}^{\bullet}(\nu):\left(D R^{\bullet}(M)\right)_{2} \rightarrow\left(D R^{\bullet+k}(M)\right)_{2}$ are defined, bounded, and smooth in $\nu \in$ $\mathbb{R}^{2} \backslash(0,0)$. (It is proved above.) For example, $d G_{\lambda}^{\bullet}(\nu)$ and $\delta G_{\lambda}^{\bullet}(\nu)$ are smooth in $\nu$. According to $(3.24)$ we have $\partial_{\gamma}\left(d G_{\lambda}^{\bullet}(\nu)\right)=d \partial_{\gamma} G_{\lambda}^{\bullet}(\nu), \partial_{\gamma}\left(\delta G_{\lambda}^{\bullet}(\nu)\right)=\delta \partial_{\gamma} G_{\lambda}^{\bullet}(\nu)$.

The operators $d G_{\lambda}^{\bullet}(\nu)$ are defined for $\lambda \notin \operatorname{Spec}\left(\Delta_{\nu}^{\bullet}\right)$. From (3.24) and (3.25) we get

$$
\begin{aligned}
\left.\partial_{\gamma}\left(d G_{\lambda}^{\bullet}(\nu)\right)\right|_{\gamma=0} & =d\left(\left.\partial_{\gamma} G_{\lambda}^{\bullet}(\nu)\right|_{\gamma=0}\right)= \\
& =-\left(\lambda-\lambda_{1}\right)^{-2} d\left(\left.\left.\frac{\partial}{\partial B} R\left(\left(\lambda-\lambda_{1}\right)^{-1}, B\right)\right|_{B=G_{\lambda_{1}}\left(\nu_{0}\right)} \partial_{\gamma} G_{\lambda_{1}}^{\bullet}(\nu)\right|_{\gamma=0}\right)
\end{aligned}
$$


for a $C^{\infty}$-local map $\left(\mathbb{R}_{\gamma}^{1}, 0\right) \rightarrow\left(\mathbb{R}^{2} \backslash(0,0), \nu_{0}\right)$, where $\lambda_{1} \in \Lambda_{\varepsilon}$ with $\left|\lambda_{1}\right|$ large enough and $\lambda \notin \operatorname{Spec}\left(\Delta_{\nu_{0}}^{\bullet}\right)$. Proposition 3.1 is proved.

3.3. Theta-functions for the Laplacians with $\nu$-transmission boundary conditions. Proofs of Theorem 3.2 and of Proposition 3.2. Let $\varepsilon$ be fixed, $0<\varepsilon<$ $\pi / 2$. The operator $\exp \left(-t \Delta_{\nu}^{\bullet}\right)$ is defined for Re $t>0, \pi / 2-\varepsilon>\arg t>-(\pi / 2-\varepsilon)$, by the integral

$$
\exp \left(-t \Delta_{\nu}^{\bullet}\right)=\frac{i}{2 \pi} \int_{\Gamma_{L, \varepsilon}} \exp (-\lambda t) G_{\lambda}^{\bullet}(\nu) d \lambda
$$

where $\Gamma_{L, \varepsilon}=\Gamma_{L, \varepsilon}^{1} \cup \Gamma_{L, \varepsilon}^{2}, \Gamma_{L, \varepsilon}^{1}=\{\lambda=-L+x \exp (i \varepsilon),+\infty>x \geq 0\}, \Gamma_{L, \varepsilon}^{2}=\{\lambda=$ $-L+x \exp (-i \varepsilon), 0 \leq x<+\infty\}, L>0$. The integral (3.26) is absolutely convergent because the operator norm in $\left(D R^{\bullet}(M)\right)_{2}$ of the operator $G_{\lambda}^{\bullet}(\nu)$ (which is bounded in $\left.\left(D R^{\bullet}(M)\right)_{2}\right)$ is estimated by $O\left(|\lambda|^{-1}\right)$ for $\lambda \in \Gamma_{L, \varepsilon}$, according to Theorem 3.1.66 This integral is independent of $L>0$ and of $\varepsilon, \pi / 2>\varepsilon>0$, for $t$ such that $|\arg t|<\pi / 2-\varepsilon$, since the spectrum of $\Delta_{\nu}^{\bullet}$ is discrete and since $\operatorname{Spec}\left(\Delta_{\nu}^{\bullet}\right) \subset \mathbb{R}_{+} \cup 0$. With the help of the inverse Mellin transform $f \rightarrow M^{-1} f$

$$
\left(M^{-1} f\right)(t):=(2 \pi i)^{-1} \int_{\operatorname{Re} s=c} \Gamma(s) t^{-s} f(s) d s
$$

it is possible to obtain the results about the asymptotic expansion for $\operatorname{Tr} \exp \left(-t \Delta_{\nu}^{\bullet}\right)$ as $\operatorname{Re} t \rightarrow+0$ (when $\pi / 2-\varepsilon>|\arg t|$ ) from the results about $\zeta_{\nu, \bullet}(-m), m \in$ $\mathbb{Z}_{+} \cup 0$, and about $\operatorname{res}_{s=s_{j}} \zeta_{\nu, \bullet}(s)$ obtained in Theorem 3.1. The integral (3.26) can be transformed as follows

$$
\exp \left(-t \Delta_{\nu}^{\bullet}\right)=\mathcal{H}^{\bullet}(\nu)+\frac{i}{2 \pi} \int_{\Gamma_{-\delta, \varepsilon}} \exp (-t \lambda) G_{\lambda}^{\bullet}(\nu) d \lambda
$$

where $\mathcal{H}^{\bullet}(\nu)$ is the kernel of the orthogonal projection operator of $\left(D R^{\bullet}(M)\right)_{2}$ onto $\operatorname{Ker} \Delta_{\nu}^{\bullet}$ and where $\delta>0$ and $\rho, \rho \geq \delta$, is such that $\operatorname{Spec}\left(\Delta_{\nu}^{\bullet}\right) \cap(0, \rho]=\emptyset$. The operator $\exp \left(-t \Delta_{\nu}^{\bullet}\right)$ for $|\arg t|<\pi / 2-\varepsilon$ can be represented as follows (where $\Gamma$ is

\footnotetext{
${ }^{66}$ The constant factor in this estimate depends on $\varepsilon$.
} 
the same as in (3.18) and $c>0)$ :

$$
\begin{aligned}
& \exp \left(-t \Delta_{\nu}^{\bullet}\right)=\mathcal{H}^{\bullet}(\nu)+\frac{i}{2 \pi} \int_{\Gamma_{-\delta, \varepsilon}} \exp (-t \lambda) G_{\lambda}^{\bullet}(\nu) d \lambda= \\
&= \mathcal{H}^{\bullet}(\nu)+\frac{i}{2 \pi} \int_{\Gamma_{-\delta, \varepsilon}}(2 \pi i)^{-1} G_{\lambda}^{\bullet}(\nu)\left(\int_{\operatorname{Re} s=c}(\lambda t)^{-s} \Gamma(s) d s\right) d \lambda= \\
&= \mathcal{H}^{\bullet}(\nu)+(2 \pi i)^{-1} \int_{\operatorname{Re} s=c} t^{-s} \Gamma(s)\left(\frac{i}{2 \pi} \int_{\Gamma_{-\delta, \varepsilon}} \lambda^{-s} G_{\lambda}^{\bullet}(\nu) d \lambda\right) d s= \\
&= \mathcal{H}^{\bullet}(\nu)+(2 \pi i)^{-1} \int_{\operatorname{Re} s=c} t^{-s} \Gamma(s)\left(\frac{i}{2 \pi} \int_{\Gamma} \lambda^{-s} G_{\lambda}^{\bullet}(\nu) d \lambda\right) d s= \\
&=\mathcal{H}^{\bullet}(\nu)+(2 \pi i)^{-1} \int_{\operatorname{Re} s=c} \Gamma(s) t^{-s} T_{-s}(\nu) d s .
\end{aligned}
$$

Here, the integration is over Re $s=c$ from $c-i \infty$ to $c+i \infty$ (where $c>0$ ). The operator $T_{-s}(\nu)$ for Re $s>0$ is defined by the integral (3.18). The transformations we apply in (3.27) are correct by the Fubini theorem since the estimate

$$
\left\|G_{\lambda}^{\bullet}(\nu)\right\|_{2}<C \cdot|\lambda|^{-1}, \quad \lambda \in \Gamma_{-\delta, \varepsilon},
$$

is satisfied by the operator norm of $G_{\lambda}^{\bullet}(\nu)$ in $\left(D R^{\bullet}(M)\right)_{2}$ and since for Re $s>0$ the gamma-function can be estimated as follows. We have

$$
\Gamma(s)=\int_{0}^{\infty} t^{s-1} \exp (-t) d t=\int_{0}^{\infty} t^{s-1} \exp (i \varphi s) \exp (-t \exp (i \varphi)) d t
$$

for $\operatorname{Re} s>0$ and for an arbitrary $\varphi \in \mathbb{R}$ such that $\pi / 2>|\varphi|$. So the estimate holds for any $\varepsilon_{1}, 0<\varepsilon_{1} \leq \pi / 2$ and for $\operatorname{Re} s>0$ :

$$
|\Gamma(s)| \leq\left(\sin \varepsilon_{1}\right)^{-\operatorname{Re} s} \Gamma(\operatorname{Re} s) \exp \left(-\left(\frac{\pi}{2}-\varepsilon_{1}\right)|\operatorname{Im} s|\right) .
$$

The kernel of $\left(T_{-s}(\nu)\right)_{x_{1}, x_{2}}$ is continuous in $\left(x_{1}, x_{2}\right) \in \bar{M}_{j_{1}} \times \bar{M}_{j_{2}}$ for $\operatorname{Re} s>n / 2$ (according to Theorem 3.1). The equality (3.27) holds also for $c=\operatorname{Re} s>n / 2$. For such $s$ the integral $\int_{\operatorname{Re} s=c} \Gamma(s) t^{-s}\left(T_{-s}(\nu)\right)_{x_{1}, x_{2}} d s$ is absolutely convergent (by Proposition 3.5 below and by (3.28)). Hence it defines a continuous on $\bar{M}_{j_{1}} \times \bar{M}_{j_{2}}$ kernel. So the kernel $E_{t, x_{1}, x_{2}}^{\bullet}(\nu)$ of $\exp \left(-t \Delta_{\nu}^{\bullet}\right)$ is continuous on $\bar{M}_{j_{1}} \times \bar{M}_{j_{2}}$ because we have

$$
E_{t, x_{1}, x_{2}}^{\bullet}(\nu)=\mathcal{H}^{\bullet}(\nu)_{x_{1}, x_{2}}+(2 \pi i)^{-1} \int_{\operatorname{Re} s=c} \Gamma(s) t^{-s}\left(T_{-s}(\nu)\right)_{x_{1}, x_{2}} d t,
$$

where $c>n / 2$. (The integral in (3.29) converges uniformly with respect to $x_{1}, x_{2}$ for any fixed $c>n / 2$ by Proposition 3.5.)

From the functional equation $\Gamma(s)=s^{-1}(s+1)^{-1} \ldots(s+l-1)^{-1} \Gamma(s+l)$ it follows that $|\Gamma(s)|$ for Re $s>-l$ is also estimated by $\exp \left(-\left(\pi / 2-\varepsilon_{1}\right)|\operatorname{Im} s|\right)$ as $|\operatorname{Im} s| \rightarrow$ $\infty$ (with any fixed $\left.\varepsilon_{1}, 0<\varepsilon_{1} \leq \pi / 2\right)$. The operator $\exp \left(-t \Delta_{\nu}^{\bullet}\right)$ for Re $t>0$ is a 
trace class operator. Namely its kernel is continuous on $\bar{M}_{j_{1}} \times \bar{M}_{j_{2}}$ (as it follows from (3.29)). Hence it is a trace class operator and its trace is equal to the sum of the integrals over the diagonals $\bar{M}_{j}$ of the corresponding densities (according to Proposition 3.8 below).

The theta-function $\theta_{\nu, \bullet}(t)$ for $\Delta_{\nu}^{\bullet}$ is defined as the trace of $\exp \left(-t \Delta_{\nu}^{\bullet}\right)$ for $\operatorname{Re} t>0$. The analogous theta-function $\theta_{\nu, \bullet}\left(t ; p_{j}\right)$ is defined as the trace $\operatorname{Tr}\left(p_{j} \exp \left(-t \Delta_{\nu}^{\bullet}\right)\right)$ for Re $t>0$ (where $p_{j}:\left(D R^{\bullet}(M)\right)_{2} \rightarrow\left(D R^{\bullet}\left(M_{j}\right)\right)_{2} \hookrightarrow\left(D R^{\bullet}(M)\right)_{2}$ is the composition of the natural restriction and of the prolongation by zero). Proposition 3.8 claims that $\theta_{\nu, \bullet}\left(t ; p_{j}\right)$ is equal to the integral over $\bar{M}_{j}$ of the density $\operatorname{tr}\left(*_{x_{2}} i_{M_{j}}^{*} E_{t, x_{1}, x_{2}}^{\bullet}(\nu)\right)$.

The zeta-function $\zeta_{\nu, \bullet}(s)$ is defined by $(2.8)$ for $\operatorname{Re} s>n / 2(n:=\operatorname{dim} M)$. It is equal to $\operatorname{Tr} T_{-s}(\nu)$ for $\operatorname{Re} s>n / 2$ (according to Theorem 3.1 and to Proposition 3.9). The zeta-function $\zeta\left(s ; p_{j}\right):=\operatorname{Tr}\left(p_{j} T_{-s}(\nu)\right)$ is equal for such $s$ to the integral over the diagonal $i\left(\bar{M}_{j}\right) \hookrightarrow \bar{M}_{j} \times \bar{M}_{j}$ of the density, corresponding to the restriction of the kernel $T_{-s}(\nu)$ to $i\left(\bar{M}_{j}\right)$. The integral of this density over $\bar{M}_{j}$ can be represented as the sum of the integrals of densities on $\bar{M}_{j}$ and on $\partial M_{j}$ (they are defined by the parametrix (3.3) and can be continued to meromorphic functions on the whole complex plane $\mathbb{C} \ni s$ ) and of a density on $\bar{M}_{j}$, which is holomorphic for $\operatorname{Re} s>$ $(n-m) / 2$. (This assertion follows from the proof of Theorem 3.1.) The contour of the integration in (3.27) can be moved to Re $s=a$ for an arbitrary $a$ such that $(-2 a) \notin \mathbb{Z}_{+} \cup 0$ (according to the estimates of $|\Gamma(s)|$ as $|\operatorname{Im} s| \rightarrow+\infty$ and to Proposition 3.4 below). Then it follows from (3.27) that

$$
\begin{aligned}
\theta_{\nu, \bullet}\left(t ; p_{j}\right)=\sum t^{-(n-k) / 2} \operatorname{res}_{s=s_{k}}\left(\Gamma(s) \zeta_{\nu, \bullet}\left(s ; p_{j}\right)\right)+ \\
+(2 \pi i)^{-1} \int_{\operatorname{Re} s=a} t^{-s} \Gamma(s) \zeta_{\nu, \bullet}\left(s ; p_{j}\right) d s+\operatorname{Tr}\left(p_{j} \mathcal{H} \bullet(\nu)\right)
\end{aligned}
$$

where the sum is over $k$ such that $s_{k}:=(n-k) / 2>a$. The estimate of the integral over Re $s=a$ in (3.30) is obtained with the help of (3.28) and (3.40) as follows. For $\operatorname{Re} t>0,|\arg t|<\pi / 2-\varepsilon(\varepsilon, 0<\varepsilon<\pi / 2$, is fixed) and for Re $s=a$ the estimate is satisfied:

$$
\begin{gathered}
\left|t^{-s} \Gamma(s) \zeta_{\nu, \bullet}\left(s ; p_{j}\right)\right|<\left(\sin \frac{\varepsilon}{4}\right)^{-(a-[a])}|\Gamma(a)||t|^{-a} \exp \left(-\frac{\varepsilon}{2}|\operatorname{Im} s|\right) C\left(a, \frac{\varepsilon}{4}\right) \times \\
\times\left\{c_{\varepsilon / 4}^{2(a-1)} \Gamma\left(2(1-a), c_{\varepsilon / 4} \rho^{1 / 2}\right)+\max \left(\rho^{-a}, 1\right)\left(1+\sum\left|s_{k}-a\right|^{-1}\right)\right\}
\end{gathered}
$$

where the sum is over $k<n-2 a$. The constants $C(a, \varepsilon / 4), c_{\varepsilon / 4}$ in (3.31) and the function $\Gamma(u ; x)$ are as in Proposition 3.4, (3.40). The latter estimate is a consequence of $(3.28)$ and $(3.40))$, where $\varepsilon_{1}$ and $\varepsilon$ are replaced by $\varepsilon / 4$. We see that

$$
\left|\int_{\operatorname{Re} s=a} t^{-s} \Gamma(s) \zeta_{\nu, \bullet}\left(s ; p_{j}\right) d s\right|<C_{1}(\varepsilon, a)|t|^{-a}
$$


where $\operatorname{Re} t>0,|\arg t|<\pi / 2-\varepsilon, \pi / 2>\varepsilon>0, a<0$, and $(-2 a) \notin \mathbb{Z}_{+}$. The assertions of Theorem 3.2 about the asymptotic expansion $(3.1)$ for $\theta_{\nu, \bullet}\left(t ; p_{j}\right)$ (relative to $t \rightarrow+0$ when $|\arg t|<\pi / 2-\varepsilon$ ) follow from the equality (3.30) and from the estimate (3.32). The estimates analogous to (3.40) below and to (3.32) are satisfied also by the analytic continuation to $\mathbb{C} \ni s$ of the densities (on $\bar{M}_{j}$ and on $\partial M_{j}$ ) defined by the parametrix $P_{\lambda}^{m}(\nu)$ (as in Proposition 3.5 below). Thus we see that the equalities between the densities in the integral representation for the coefficients of the expansion (3.1) and the corresponding densities for the residues and the values of $\zeta_{\nu, \bullet}\left(s ; p_{j}\right)$ are satisfied.

The uniform with respect to $\nu \in \mathbb{R}^{2} \backslash(0,0)$ estimate (3.2) for the traces of $\exp \left(-t \Delta_{\nu}^{\bullet}\right)$ (for a fixed $t$, Re $t>0$ ) follows from (3.30) and (3.31) because for $a=-m-1 / 4, m \in \mathbb{Z}_{+}, m>>1$, the integral over Re $s=a$ on the right in (3.30) is absolutely convergent. The estimate ${ }^{67}$ (3.31) and the equality (3.30) provide us with the uniform in $\nu$ upper estimate for $\operatorname{Tr}\left(p_{j} \exp \left(-t \Delta_{\nu}^{\bullet}\right)\right), \nu \in \mathbb{R}^{2} \backslash(0,0)$. Indeed, the estimate ${ }^{68} \operatorname{dim} \operatorname{Ker} \Delta_{\nu}^{\bullet}<C$ is satisfied uniformly with respect to $\nu$. The formulas $q_{-n+k}=\operatorname{res}_{s=s_{k}}\left(\Gamma(s) \zeta_{\nu, \bullet}\left(s ; p_{j}\right)\right)+\delta_{n, k} \operatorname{Tr}\left(p_{j} \mathcal{H}^{\bullet}(\nu)\right)$ for the coefficients $q_{-n+k}$ of the asymptotic expansion (3.1) are consequences of (3.30) for $a=-m-1 / 4$, where $m \in \mathbb{Z}_{+}$. For $a=-m-1 / 4$ the absolute value of the integral over Re $s=a$ in (3.30) is estimated (with the help of (3.31)) by $C|t|^{m+1 / 4}$ uniformly with respect to $\nu \in \mathbb{R}^{2} \backslash(0,0)$ (where $\operatorname{Re} t>0,|\arg t|<\pi / 2-\varepsilon, \pi / 2>\varepsilon>0$ and $\varepsilon$ is an arbitrary but fixed). So it holds

$$
\theta_{\nu, \bullet}\left(t ; p_{j}\right)=\sum_{k=0}^{n+2 m-1} q_{-n+k} t^{-(n-k) / 2}+\left\{q_{-n+2 m} t^{m}+O\left(|t|^{m+1 / 4}\right)\right\} .
$$

The latter two terms in (3.33) are $O\left(|t|^{m}\right)$ relative to $t \rightarrow+\infty$ uniformly with respect to $\nu \neq(0,0)$ (for $|\arg t|<\pi / 2-\varepsilon$ ). The statements about the structure of the values and the residues of $\zeta_{\nu, \bullet}\left(s ; p_{j}\right)$ (Theorem 3.1) provide us with the desired information about coefficients $q_{-n+k}$ in (3.1). These values and residues (up to $\left.\delta_{n, k} \operatorname{Tr}\left(p_{j} \mathcal{H}^{\bullet}(\nu)\right)\right)$ are the sums of the integrals over $M_{j}, \partial M$, and $N$ of the densities which are defined by the absolutely convergent integrals of the components $a_{-2-k}$ and $d_{-2-k+1}$ ([Se2], Theorem 2, and the proof of Theorem 3.1 above). The latter symbols are defined by (3.4), (3.11), and (3.10). These integrals are smooth in $\nu \in \mathbb{R}^{2} \backslash(0,0)$. Hence the coefficients $q_{-n+k}$ in (3.1) are smooth in $\nu \neq(0,0)$ (and are invariant under $\nu \rightarrow c \nu, c \neq 0)$. Theorem 3.2 is proved.

\footnotetext{
${ }^{67}$ For $a<0$ the function $\Gamma\left(2(1-a), c_{\varepsilon / 4} \rho^{1 / 2}\right)$ tends to $\Gamma(2(1-a))$ as $\rho \rightarrow+0$ and so it is bounded for $0<\rho<1$.

${ }^{68}$ It follows from the exact sequence (1.14) (where $Z_{j}:=Z \cap \partial M_{j}$ ) and from Lemma 1.1 that

$$
\operatorname{dim} \operatorname{Ker} \Delta_{\nu}^{\bullet}=\operatorname{dim} H^{\bullet}\left(M_{\nu}, Z\right) \leq \sum_{j} \operatorname{dim} H^{\bullet}\left(M_{j}, N \cup Z_{j}\right)+\operatorname{dim} H^{\bullet}(N) .
$$
}


Remark 3.2. The coefficients $q_{-n+k}$ of (3.1) for $0 \leq k \leq m$ are completely defined (according to (3.33) and to Remark 3.1) by an arbitrary parametrix $P_{\lambda}^{m}(\nu)(3.3)$ for $\left(\Delta_{\nu}^{\bullet}-\lambda\right)^{-1}$.

Proof of Proposition 3.2. The parametrix ${ }^{69} P_{t, x, y}^{\bullet(m)}(\nu)$ for $E_{t, x, y}^{\bullet}(\nu)$ (defined by (2.126)) is such that it is smooth in $(x, y) \in \bar{M}_{j_{1}} \times \bar{M}_{j_{2}}$ and in $\nu \in \mathbb{R}^{2} \backslash(0,0)$. The $\nu$-transmission boundary conditions $(1.27)$ are satisfied for $\left(\Delta_{\nu, x}^{\bullet}\right)^{k} P_{t, x, y}^{\bullet(m)}(\nu)$ (i.e., the image of $\left(D R^{\bullet}(M)\right)_{2}$ under the action of the operator with the kernel $P_{t, x, y}^{\bullet(m)}(\nu)$ belongs to $D\left(\left(\Delta_{\nu}^{\bullet}\right)^{k}\right)$ for an arbitrary $\left.k \in \mathbb{Z}_{+}\right)$. The uniform with respect to $\nu \in \mathbb{R}^{2} \backslash(0,0)$ estimates (2.127), (2.128) are satisfied and for $x$ from an appropriate neighborhood $U$ of $N \subset M$ we have $\left(\partial_{t}+\Delta_{\nu, x}^{\bullet}\right) P_{t, x, y}^{\bullet(m)}(\nu) \equiv 0$ (where $U$ is independent of $\nu$ ).

Set $r_{t, x, y}^{(m)}(\nu):=\left(\partial_{t}+\Delta_{\nu, x}^{\bullet}\right) P_{t, x, y}^{\bullet(m)}(\nu)$. Then the estimates are satisfied for any $k \in$ $\mathbb{Z}_{+} \cup 0$

$$
\left|\Delta_{\nu, x}^{k} r_{t, x, y}^{(m)}(\nu)\right|<C_{m, k} t^{-n / 2+m-k}
$$

where $C_{m, k}$ is independent of $\nu \in \mathbb{R}^{2} \backslash(0,0)$ and of $t \in(0, T](n:=\operatorname{dim} M)$.

The kernel $E_{t, x, y}^{\bullet}(\nu)$ can be represented as the Volterra series ${ }^{70}$

$$
\begin{aligned}
& E_{t, x_{1}, x_{2}}^{\bullet}(\nu)= \\
& \quad=\sum_{k \geq 0}(-t)^{k} \int_{\Delta_{k}} \int_{\left(y_{1}, \ldots, y_{k}\right) \in\left(\bar{M}_{1} \cup \bar{M}_{2}\right)^{k}} P_{\sigma_{0} t, x_{1}, y_{1}}^{\bullet(m)}(\nu) r_{\sigma_{1} t, y_{1}, y_{2}}^{(m)}(\nu) \ldots r_{\sigma_{k} t, y_{k}, x_{2}}^{(m)}(\nu),
\end{aligned}
$$

where $\Delta_{k}=\left\{\left(\sigma_{0}, \ldots, \sigma_{k}\right): 0 \leq \sigma_{i} \leq 1, \sum \sigma_{i}=1\right\}$ (and the scalar product $\operatorname{tr}\left(\omega_{1} \wedge * \omega_{2}\right)$ with the values in densities on $M$ is implied in (3.35)). The proof of (3.35) (or of $(2.137))$ is given in the proof of Proposition 2.21.

Let $\varphi: I \rightarrow \mathbb{R}^{2} \backslash(0,0), \nu=\varphi(\gamma)$, be a $C^{\infty}$-map (where $\left.\gamma \in[-a, a]=: I\right)$. Then the only term in $P_{t,\left(x_{1}, x^{\prime}\right),\left(y_{1}, y^{\prime}\right)}^{\bullet(\nu)}(\nu)$ depending on $\gamma$ is

$$
E_{N, t} \otimes \psi\left(x_{1}\right) E_{I, t}(\nu) \varphi\left(y_{1}\right)=: E_{N, I}^{\bullet}(\nu)
$$

but it does not depend on $m$. (Here $E_{I, t}$ corresponds to $\Delta_{I}$ with the Dirichlet boundary conditions on $\partial I, \varphi, \psi \in C_{0}^{\infty}(I \backslash \partial I), \psi \equiv 1$ in a neighborhood of $\operatorname{supp} \varphi \subset$ $I \backslash \partial I, \varphi\left(x_{1}\right) \equiv 1$ for $x_{1} \in[-1 / 2,1 / 2]$ and $\varphi, \psi$ are even: $\varphi\left(-x_{1}\right)=\varphi\left(x_{1}\right), \psi\left(-x_{1}\right)=$ $\psi\left(x_{1}\right)$.) So, as it follows from the explicit formulas $(2.40)$ for $\left(G_{I}(\nu)\right)_{x_{1}, y_{1}}$ (and from

\footnotetext{
${ }^{69}$ The properties of such a parametrix are summarized in Proposition 2.21. For the sake of brevity the proof of Proposition 3.2 is given in the case of $\partial M=\emptyset$. The terms of $P_{t}^{(m)}(\nu)$, connected with the Dirichlet and the Neumann boundary conditions on the components of $\partial M$, are independent of $\nu$ and the proof in the case of $\partial M \neq \emptyset$ does not contain any additional difficulties.

${ }^{70}$ This series was used in the case of a closed manifold $M$ in $[\mathrm{BGV}], 2.4,2.7$. See also the formula (2.137) above.
} 
the analogous formulas $(2.54)$ and $(2.55)$ for $\left.\left(E_{I, t}(\nu)\right)_{x_{1}, y_{1}}\right)$, the uniform with respect to $\gamma$ estimates are satisfied for any $k, q \in \mathbb{Z}_{+}$

$$
\left|\partial_{\gamma}^{k} r_{t, x, y}^{(m)}(\nu)\right|<c_{(q, k)} t^{-n / 2+q} .
$$

because they are true for $\left(\partial_{t}+\Delta_{x^{\prime}}+\Delta_{x_{1}}\right)\left\{\left(E_{N, t}\right)_{x^{\prime}, y^{\prime}} \otimes \psi\left(x_{1}\right)\left(E_{I, t}(\nu)\right)_{x_{1}, y_{1}} \varphi\left(y_{1}\right)\right\}$ and for $\left(\partial_{t}+\Delta_{x^{\prime}}+\Delta_{x_{1}}\right)\left\{\left(E_{N, t}\right)_{x^{\prime}, y^{\prime}} \otimes \psi\left(x_{1}\right)\left(\sigma_{1}^{*} E_{I, t}(\nu)\right)_{x_{1}, y_{1}} \varphi\left(y_{1}\right)\right\}$ (where $\sigma_{1}$ is the reflection of $I=[-1,1]$ with respect to $0 \in I$ which acts on the variable $\left.x_{1}\right)$. The kernels $\partial_{\gamma}^{k} E_{N, I}^{\bullet}(\nu)$ are the linear combinations of these two kernels with the coefficients independent of $x$ and $y$. (These coefficients are smooth in $\gamma$ ). The estimate (3.37) is satisfied for $t \in(0, T)$ and for an arbitrary $q \in \mathbb{Z}_{+}$uniformly with respect to $\gamma$, because if $\partial_{x_{1}} \psi\left(x_{1}\right) \neq 0$ then $\rho\left(x_{1}, \operatorname{supp} \varphi\right)>\delta>0$.

Let $D R_{M}^{\bullet}(l)$ be the space of forms on $M$ of a class $C^{l}$ (i.e., of forms with $l$ continuous derivatives on $M$ ) equiped with a $C^{l}$-norm. ${ }^{71}$ Let $D R_{M, N}^{\bullet}(l):=D R_{(l)}^{\bullet}\left(\bar{M}_{1}\right) \oplus$ $D R_{(l)}^{\bullet}\left(\bar{M}_{2}\right)$ be the space of pairs $\left(\omega_{1}, \omega_{2}\right)$ of forms $\omega_{j}$ of a class $C^{l}$ on $\bar{M}_{j}$ with a $C^{l_{-}}$ norm. The operators with the kernels $P_{t, x, y}^{\bullet(m)}(\nu)$ for $\nu \in \mathbb{R}^{2} \backslash(0,0)$ and the operators corresponding to $\partial_{\gamma}^{k} P_{t, x, y}^{\bullet(m)}\left(\nu(\gamma)\right.$ ) (for a fixed $k \in \mathbb{Z}_{+} \cup 0$ ) are families of uniformly (with respect to $\nu$ and to $t \in(0, T])$ bounded operators acting from $D R_{M}^{\bullet}(l)$ into $D R_{M, N}^{\bullet}(l)$. For the operators, corresponding to the interior terms in $P_{t, x, y}^{\bullet(m)}(\nu)$ this assertion is proved in $[\mathrm{BGV}]$, Theorem 2.29, Lemma 2.49. This proof uses that this statement is local in $x \in M$ (for a closed $M$ ) and it uses also the explicit definition of $P_{i n t}^{(m)}$ over a geodesic ball $\exp _{x} B \subset M$ (where $B$ is a ball $\|v\| \leq c$ in $T_{x} M$ and $\exp _{x}$ is the exponential map for $\left(M, g_{M}\right)$ from $\left.T_{x} M\right)$.

The kernel (3.36) (i.e., the term of $P_{t, x, y}^{\bullet(m)}(\nu)$ corresponding to the interior boundary $N$ ) is equal (up to the factor $\left.\psi\left(x_{1}\right) \varphi\left(y_{1}\right)\right)$ to a linear combination given by (2.54) and (2.55) of the kernels $E_{t}^{\bullet}$ for $N \times I$ and $\sigma_{1}^{*} E_{t}^{\bullet}\left(\sigma_{1}\right.$ is the mirror symmetry with respect to $N \times 0) .{ }^{72}$ Its coefficients depend on $\left(j_{1}, j_{2}, \nu\right)$, where $(x, y) \in \bar{M}_{j_{1}} \times \bar{M}_{j_{2}}$. These coefficients and their derivatives of a fixed order on $\gamma$ are uniformly bounded.

For a closed $N$ the operators defined by $\left(P_{N}^{(m)}\right)_{t}:=\left(P_{i n t, N}^{(m)}\right)_{t}$ are uniformly bounded for $0<t \leq T$ with respect to a $C^{l}$-norm in the space $D R_{N}^{\bullet}(l)$ of $C^{l}$-smooth forms on $N$. The equality (3.35) is satisfied by $E_{N, t}^{\bullet}, P_{N, t}^{\bullet(m)}$ and $r_{N, t}^{(m)}$. Since the estimate (3.38) below (as well as the analogous estimate (3.34)) is satisfied by $r_{N, t}^{(m)}$ (where $n$ is replaced by $n-1)$ we see that the series of operators on the right in (3.35) is

${ }^{71}$ This norm corresponds to a smooth partition of unity $\left\{\varphi_{i}\right\}$ subordinate to a finite cover $\left\{U_{i}\right\}$ of $\bar{M}_{1}$ and of $\bar{M}_{2}$ by coordinate charts (i.e., $\left.\varphi_{i} \in C_{0}^{\infty}\left(U_{i}\right)\right)$. For $v \in D R_{M, N}^{\bullet}(l)$ its $C^{l}$-norm $\|v\|_{l}$ is equal to $\sum \sup _{x \in U_{i}} \sup _{|\alpha| \leq l}\left|D_{x}^{\alpha}\left(\varphi_{i} v\right)\right|$ i.e., to the sum of the suprema of partial derivatives of orders $\leq l$. The $C^{l}$-norm for an arbitrary smooth finite cover $\left\{U_{i}^{\prime}\right\}$ and for a partition of unity $\left\{\varphi_{i}^{\prime}\right\}$ subordinate to $\left\{U_{i}^{\prime}\right\}$ is equivalent to the one defined by $\left\{U_{i}\right\}$ and by $\left\{\varphi_{i}\right\}$.

${ }^{72}$ The operators $\partial_{\gamma}^{k} P^{(m)}(\nu)=\partial_{\gamma}^{k} E_{N, I}(\nu)$ for $k \in \mathbb{Z}_{+}$are expressed similarly. 
convergent for $m \geq(n+l-1) / 2$ relative to a $C^{l}$-norm in $D R_{N}^{\bullet}(l)$. Hence the sum of this series defines a family of the operators $E_{N, t}^{\bullet}$ in $D R_{N}^{\bullet}(l)$ bounded uniformly with respect to $t \in(0, T]$. The analogous assertion is also true for a family of operators defined by the kernels $\psi\left(x_{1}\right)\left(E_{I, t}^{\bullet}\right)_{x_{1}, y_{1}} \varphi\left(y_{1}\right)$ acting on smooth forms with compact supports on $(I, \partial I)$ (with respect to a $C^{l}$-norm). So the kernels $E_{N, I}^{\bullet}(\nu)_{t,\left(x_{1}, x^{\prime}\right),\left(y_{1}, y^{\prime}\right)}$ and $\partial_{\gamma}^{q} E_{N, I}^{\bullet}(\nu(\gamma))_{t,\left(x_{1}, x^{\prime}\right),\left(y_{1}, y^{\prime}\right)}$ (for a fixed $q \in \mathbb{Z}_{+}$) define collections of uniformly (in $t \in(0, T]$ and $\nu$ or in $t$ and $\gamma)$ bounded with respect to a $C^{l}$-norm operators from $D R_{M}^{\bullet}(l)$ into $D R_{M, N}^{\bullet}(l)$.

The kernel $r_{t, x, y}^{(m)}(\nu)$ is smooth on $M \times \bar{M}_{j}$ according to the definition (2.126) of $P_{t, x, y}^{\bullet(m)}(\nu)$. The $C^{l}$-norm of $r_{t, x, y}^{(m)}(\nu)$ on each $M \times \bar{M}_{j}$ satisfies the estimate (analogous to $(3.34))$

$$
\left\|r_{t, x, y}^{(m)}(\nu)\right\|_{l} \leq C_{l} t^{-n / 2+m-l / 2} .
$$

uniformly with respect to $\nu \in \mathbb{R}^{2} \backslash(0,0)$ and to $t \in(0, T]$. The estimates analogous to (3.37) and to (2.129) are satisfied uniformly with respect to $\nu$ and to $t \in(0, T]$ also by the $C^{l}$-norms of $\partial_{\gamma}^{k} r_{t, x, y}^{(m)}(\nu)$ on each $M \times \bar{M}_{j}$ for any $k, q \in \mathbb{Z}_{+}$:

$$
\left\|\partial_{\gamma}^{k} r_{t, x, y}^{(m)}(\nu)\right\|_{l} \leq c_{(q, k, l)} t^{-n / 2+q}
$$

Leibnitz's rule claims that $P_{t, x, y}^{(m)}(\nu(\gamma))$ is a bounded operator from the space of $C^{p}$-maps $\omega:[-a, a] \rightarrow D R_{M}^{\bullet}(l)$ (equiped with the norm $\sum_{i=0}^{p} \sup _{\gamma \in[-a, a]}\left\|\partial_{\gamma}^{i} \omega\right\|_{l}$ ) into $C^{p}\left([-a, a], D R_{M, N}^{\bullet}(l)\right)$. Indeed, the kernel $\partial_{\gamma}^{k} P_{t, x, y}^{(m)}(\nu(\gamma))$ depends smoothly on $\gamma \in$ $[-a, a]$ on $\bar{M}_{j_{1}} \times \bar{M}_{j_{2}}$ for $k \in \mathbb{Z}_{+} \cup 0$, since $P_{t, x, y}^{(m)}(\nu):=\left(P_{i n t}^{(m)}\right)_{t, x, y}+E_{N, I}^{\bullet}(\nu)$ and since $E_{N, I}^{\bullet}(\nu)$ is smooth in $\nu \in \mathbb{R}^{2} \backslash(0,0)$. For $0<t \leq T$ the operators $\left(P_{t}^{(m)}(\nu(\gamma))\right)$ from $C^{p}\left([-a, a], D R_{M}^{\bullet}(l)\right)$ into $C^{p}\left([-a, a], D R_{M, N}^{\bullet}(l)\right)$ are uniformly bounded, because $\partial_{\gamma}^{k} P_{t}^{(m)}$ (for a fixed $k \in \mathbb{Z}_{+} \cup 0$ ) are the operators from $D R_{M}^{\bullet}(l)$ into $D R_{M, N}^{\bullet}(l)$ bounded uniformly in $\gamma$ and $t, 0<t \leq T$, with respect to a $C^{l}$-norm. Hence, according to $(3.38),(3.39)$, and to the fact that the volume of $\Delta_{k}$ is equal to $(k !)^{-1}$, the series (3.35) for the derivative $\partial_{\gamma}^{p} E_{t, x_{1}, x_{2}}(\nu(\gamma))$ is convergent in the $C^{l}$-norm on $\cup\left(\bar{M}_{j_{1}} \times \bar{M}_{j_{2}}\right)$ for $m>(n+l) / 2$. (The number $m$ in the definition $P^{(m)}$ is greater than $(n+l) / 2$.)

This proves that $\partial_{\gamma}^{k} E_{t, x_{1}, x_{2}}^{\bullet}(\nu(\gamma))$ is $C^{\infty}$-smooth on $\bar{M}_{j_{1}} \times \bar{M}_{j_{2}}$. (For instance, for $k=0$ this proves that $E_{t, x_{1}, x_{2}}^{\bullet}(\nu)$ is $C^{\infty}$-smooth on $\bar{M}_{j_{1}} \times \bar{M}_{j_{2}}$.)

So the restrictions $i_{j}^{*} E_{t, x, x}^{\bullet}(\nu(\gamma))$ to the diagonals $i_{j}: \bar{M}_{j} \hookrightarrow \bar{M}_{j} \times \bar{M}_{j}$ are $C^{\infty}$ smooth double forms on $\bar{M}_{j}$ which are $C^{\infty}$-smooth in $\gamma$. Since $r_{m}(t, \nu)$ in $(3.1)$ are $O\left(t^{(m+1) / 2}\right)$ uniformly with respect to $\nu \in \mathbb{R}^{2} \backslash(0,0)$ and since $q_{i}$ are $C^{\infty}$ - 
smooth in $\nu$ we see that the asymptotic series (3.1) can be differentiated on $\gamma$. Actually, the equality (3.35) holds for $E_{t}^{\bullet}(\nu), P_{t}^{\bullet(m)}(\nu)$, and $r_{t}^{(m)}(\nu)$. The kernel $r_{t}^{(m)}(\nu)$ satisfies the estimates $(3.34),(3.37)$, and (3.39) and the kernel $P_{t}^{\bullet(m)}(\nu(\gamma))$ defines a family of uniformly with respect to $t \in(0, T]$ and to $\gamma$ bounded operators from $C^{p}\left([-a, a], D R_{M}^{\bullet}(l)\right)$ into $C^{p}\left([-a, a], D R_{M, N}^{\bullet}(l)\right)$. Hence the power terms $t^{(-n+j) / 2}, 0 \leq j \leq 2 m$, in the asymptotic expansion of $\int_{M_{j}} \operatorname{tr} i_{j}^{*} \partial_{\gamma} E_{t}^{\bullet}(\nu)$ as $t \rightarrow+0$ are equal to the appropriate terms in the asymptotic expansion of $\int_{M_{j}} i_{j}^{*} \partial_{\gamma} P_{t}^{\bullet(m)}(\nu)$. (The kernel $\partial_{\gamma}\left(E_{t}^{\bullet}(\nu)-P_{t}^{\bullet(m)}(\nu)\right)_{x_{1}, x_{2}}$ is $O\left(t^{-n / 2+m+1}\right)$, according to (3.35).) But the coefficients $q_{i}, 0 \leq i \leq 2 m$, in (3.1) are completely defined by $i_{j}^{*} P_{t}^{\bullet(m)}(\nu)$, because the kernel $\left(E_{t}^{\bullet}(\nu)-P_{t}^{\bullet(m)}(\nu)\right)_{x_{1}, x_{2}}$ is $O\left(t^{-n / 2+m+1}\right)$ uniformly with respect to $\left(x_{1}, x_{2}\right) \in \bar{M}_{j_{1}} \times \bar{M}_{j_{2}}$ and to $t \in(0, T]$, according to (3.35). Thus Proposition 3.2 is proved.

\subsection{Estimates for zeta-functions and for the corresponding kernels in ver- tical strips in the complex plane.}

Proposition 3.4. The meromorphic continuation of the zeta-function $\zeta_{\nu, \bullet}\left(s ; p_{j}\right):=$ $\operatorname{Tr}\left(p_{j} T_{-s}(\nu)\right)$ for Re $s>n / 2$ is estimated by $C(\varepsilon) \exp (\varepsilon|\operatorname{Im} s|)$ as $|\operatorname{Im} s| \rightarrow+\infty$ for any fixed $\varepsilon>0$. Namely for any $\varepsilon>0$ and for an arbitrary $a \in \mathbb{R}$ the following estimate is satisfied if $\operatorname{Re} s \geq a$ :

$$
\begin{aligned}
& \left|\zeta_{\nu, \bullet}\left(s ; p_{j}\right)\right| \leq C(a, \varepsilon) \exp (\varepsilon|\operatorname{Im} s|) \times \\
& \times\left(c_{\varepsilon}^{2(\operatorname{Re} s-1)} \Gamma\left(2(1-\operatorname{Re} s), c_{\varepsilon} \rho^{1 / 2}\right)+\max \left(\rho^{-\operatorname{Re} s}, 1\right)\left(1+\sum\left|s-s_{j}\right|^{-1}\right)\right),
\end{aligned}
$$

where $\rho>0$ is such that $\operatorname{Spec}\left(\Delta_{\nu}^{\bullet}\right) \cap(0, \rho]=\emptyset$ and the sum is over $s_{j}:=(n-j) / 2$, $-s_{j} \notin \mathbb{Z}_{+} \cup 0, s_{j} \geq a$. The constants $C(a, \varepsilon)$ and $c_{\varepsilon}$ are positive and independent of $\nu \in \mathbb{R}^{2} \backslash(0,0)$, and $\Gamma(u, x):=\int_{x}^{\infty} t^{u-1} \exp (-t) d t$ for $x>0$.

Proposition 3.5. For $\operatorname{Re} s>n / 2(n:=\operatorname{dim} M)$ and for any $\varepsilon>0$ the following estimate is satisfied (where $\rho>0$ is such that $\left.\operatorname{Spec}\left(\Delta_{\nu}^{\bullet}\right) \cap(0, \rho]=\emptyset\right)$ :

$$
\left|\left(T_{-s}(\nu)\right)_{x_{1}, x_{2}}\right| \leq C_{\varepsilon} \rho^{-\operatorname{Re} s} \exp (\varepsilon|\operatorname{Im} s|)\left(\left(\operatorname{Re} s-\frac{n}{2}\right)^{-1}+1\right) .
$$

Proof of Proposition 3.4. It is proved in Theorem 3.1 that the operator norm $\left\|G_{\lambda}^{\bullet}(\nu)\right\|_{2}$ in $\left(D R^{\bullet}(M)\right)_{2}$ of the Green function $G_{\lambda}^{\bullet}(\nu)$ for the Laplacian $\Delta_{\nu}^{\bullet}$ is estimated by $C_{\varepsilon}|\lambda|^{-1}$ for $\lambda \in \Lambda_{\varepsilon}:=\{\lambda \in \mathbb{C}, \varepsilon \leq \arg \lambda \leq 2 \pi-\varepsilon\}$, where $\varepsilon, 0<\varepsilon \leq \pi$ is fixed. The spectrum $\operatorname{Spec}\left(\Delta_{\nu}^{\bullet}\right)$ is a discrete subset of $\mathbb{R}_{+} \cup 0$ by Theorem 3.1. So the 
operator $T_{-s}(\nu)$ defined by the integral (3.18) is equal to the same integral with the contour $\Gamma$ replaced by $\Gamma_{(\varepsilon)}:=\Gamma_{1, \varepsilon} \cup \Gamma_{\rho}^{\varepsilon} \cup \Gamma_{2, \varepsilon}$,

$$
\begin{gathered}
\Gamma_{1, \varepsilon}=\{\lambda=x \exp (i \varepsilon), \infty>x \geq \rho\}, \quad \Gamma_{\rho}^{\varepsilon}=\{\lambda=\rho \exp (i \varphi), \varepsilon>\varphi>-\varepsilon\}, \\
\Gamma_{2, \varepsilon}=\{\lambda=x \exp (-i \varepsilon), \rho \leq x<\infty\} .
\end{gathered}
$$

There is a constant $c>0$ such that the principal symbol $\left(b_{2}(x, \xi)-\lambda\right)$ id of $\Delta^{\bullet}-\lambda$ id on $M$ is invertible for

$$
|\xi|^{2}>c|\lambda|
$$

in the coordinate charts $U_{l}$ (of the same finite cover $\left\{U_{l}\right\}$ of $M$ as in (3.3)). The integral (3.18) over the contour $\Gamma_{(\varepsilon)}$ (the latter one is defined by (3.42)) does not depend on $\rho$ for all $\rho>0$ such that $(0, \rho] \cap \operatorname{Spec}\left(\Delta_{\nu}^{\bullet}\right)=\emptyset$. We suppose from now on that

$$
0<\rho<(2 c+1)^{-1}
$$

and that $(0, \rho] \cap \operatorname{Spec}\left(\Delta_{\nu}^{\bullet}\right)=\emptyset$.

The kernel $\left(r_{\lambda}^{m}\right)_{x_{1}, x_{2}}$ of the operator $r_{\lambda}^{m}:=G_{\lambda}^{\bullet}(\nu)-P_{\lambda}^{m}$ for $m \geq n$ is continuous on $\left(\bar{M}_{j_{1}} \times \bar{M}_{j_{2}}\right)$ for $\lambda \in \Lambda_{\varepsilon} \backslash 0$. (The parametrix $P_{\lambda}^{m}$ is defined by (3.3).) It is estimated for $|\lambda| \geq \rho, \lambda \in \Lambda_{\varepsilon}$ (according to (3.19)) uniformly with respect to $\nu \in \mathbb{R}^{2} \backslash(0,0)$ by

$$
\left|\left(r_{\lambda}^{m}\right)_{x_{1}, x_{2}}\right|<C_{\varepsilon, \varepsilon_{1}}\left(1+|\lambda|^{1 / 2}\right)^{-(2+m)+n+\varepsilon_{1}}
$$

for any $\varepsilon_{1}>0$. Since (3.45) is satisfied for all $\lambda \in \Gamma_{(\varepsilon)}$, we have for $\operatorname{Re} s>$ $2^{-1}\left(-m+n+\varepsilon_{1}\right)$ that

$$
\begin{aligned}
&\left|\frac{i}{2 \pi} \int_{\Gamma_{(\varepsilon)}} \lambda^{-s}\left(r_{\lambda}^{m}\right)_{x_{1}, x_{2}} d \lambda\right|=\left|\frac{i}{2 \pi}\left(\sum_{j} \int_{\Gamma_{j, \varepsilon}}+\int_{\Gamma_{\rho}^{\varepsilon}}\right) \lambda^{-s}\left(r_{\lambda}^{m}\right)_{x, x} d \lambda\right| \leq \\
& \leq 2 C_{\varepsilon, \varepsilon_{1}} \exp (\varepsilon|\operatorname{Im} s|) \times \\
& \times\left\{\int_{\rho}^{\infty}|\lambda|^{-\operatorname{Re} s}\left(1+|\lambda|^{1 / 2}\right)^{-(2+m)+n+\varepsilon_{1}} d|\lambda|+\pi \rho \max \left(\rho^{-\operatorname{Re} s}, 1\right)\right\} .
\end{aligned}
$$

The estimate (3.46) claims that for the proof of (3.40) in the domain $\operatorname{Re} s \geq a$ it is enough to prove the analogous estimate for the analytical continuation of the densities on $\bar{M}_{j}, N$, and on $\partial M$, corresponding (for Re $s>n / 2$ ) to the kernel of

$$
\frac{i}{2 \pi} \int_{\Gamma_{(\varepsilon)}} \lambda^{-s} P_{\lambda}^{m} d \lambda,
$$

where $m=m(a) \in \mathbb{Z}_{+}$is sufficiently large. (These densities were introduced in the proof of Theorem 3.1, and the sum of their integrals is equal to the trace of (3.47).)

Let $\operatorname{Re} s>n / 2$ and $p_{\text {int }}^{s, \varepsilon}(x)$ be the density on $M_{j}$, corresponding to the restriction to the diagonal $i_{j}: M_{j} \hookrightarrow M_{j} \times M_{j}$ of the kernel $P_{i n t}^{s, \varepsilon}\left(x_{1}, x_{2}\right)$ of the operator 
$\int_{\Gamma_{(\varepsilon)}} \lambda^{-s}\left(p_{j} \sum_{i} \psi_{i} P_{\lambda, \text { int } U_{i}}^{m} \varphi_{i}\right) d \lambda$ (where $P_{\lambda, \text { int }}^{m}$ is defined by (3.13) and by (3.4)). Then $p_{\text {int }}^{s, \varepsilon}(x)$ can be continued to a whole complex plane $\mathbb{C} \ni s$ as a meromorphic density ([Se2], Lemma 1, or [Sh], Theorem 12.1).

Proposition 3.6. The density $p_{\text {int }}^{\text {s, }}(x)$ satisfies the following estimate for any $\varepsilon>0$ :

$$
\left|p_{\text {int }}^{s, \varepsilon}(x)\right|<C_{\varepsilon} \max \left(\rho^{-\operatorname{Re} s}, 1\right) \exp (\varepsilon|\operatorname{Im} s|) \sum\left|s-s_{k}\right|^{-1},
$$

where the sum is over $0 \leq k \leq m$ such that $\left(-s_{k}\right) \notin \mathbb{Z}_{+} \cup 0$.

Proof. The density $p_{i n t}^{s, \varepsilon}(x)$ for Re $s>n / 2$ corresponds to the sum of the integrals

$$
(2 \pi)^{-n} \sum_{j} \varphi_{j}(x) \int d \xi \frac{i}{2 \pi} \int_{\Gamma_{(\varepsilon)}} \lambda^{-s} \theta(\xi, \lambda) \sum_{j=0}^{m} a_{-2-j}(x, \xi, \lambda) d \lambda,
$$

where $a_{-k}$ is a positive homogeneous of degree $(-k)$ in $\left(\xi, \lambda^{1 / 2}\right)$ component of the symbol $s\left(\left(\Delta^{\bullet}-\lambda\right)^{-1}\right)$ in the coordinate chart $U_{i}$ defined by (3.4). The integral (3.49) is the sum of the integrals $J_{0, \varepsilon}^{s}(x)+J_{1, \varepsilon}^{s}(x)+J_{2, \varepsilon}^{s}(x)$ over the three corresponding domains:

$$
\begin{aligned}
K_{0} & :=\left\{(\xi, \lambda):|\xi|^{2} \leq 1-\rho, \lambda \in \Gamma_{(\varepsilon)},|\lambda| \leq\left(1-|\xi|^{2}\right)\right\}, \\
I_{1} & :=\left\{(\xi, \lambda):|\xi|^{2} \leq 1-\rho, \lambda \in \Gamma_{(\varepsilon)},|\lambda|>\left(1-|\xi|^{2}\right)\right\}, \\
I_{2} & :=\left\{(\xi, \lambda):|\xi|^{2}>1-\rho, \lambda \in \Gamma_{(\varepsilon)}\right\},
\end{aligned}
$$

Since $K_{0}$ is compact and since $\lambda^{-s} \theta \sum_{0}^{m} a_{-2-j}(x, \xi, \lambda)$ is continuous on $M_{j} \times K_{0}$, the density $J_{0, \varepsilon}^{s}(x)$ is holomorphic in $s \in \mathbb{C}$ and it is estimated by

$$
\begin{aligned}
& \left|J_{0, \varepsilon}^{s}(x)\right| \leq \\
& \quad \leq C_{\varepsilon} \exp (\varepsilon|\operatorname{Im} s|) \max \left(\rho^{-\operatorname{Re} s}, 1\right) \max _{M_{j} \times K_{0}} \sum_{i}\left|\varphi_{i}(x) \theta \sum_{0}^{m} a_{-2-j}(x, \xi, \lambda)\right| .
\end{aligned}
$$

The latter factor on the right in (3.51) does not depend on $s$ and $\rho$. Hence it is estimated by a constant.

Set $J_{k}^{s}(x):=J_{k, \varepsilon}^{s}(x)$ from now on. For Re $s>n / 2$ the density $J_{2}^{s}(x)$ does not change if the interior integral in (3.49) is replaced by the integral over

$$
\Gamma_{(\varepsilon),|\xi|}:=|\xi|^{2}(1-\rho)^{-1} \Gamma_{(\varepsilon)},
$$

because $\theta \sum_{0}^{m} a_{-2-j}$ is holomorphic in $\lambda$ in the domain between the contours $\Gamma_{(\varepsilon)}$ and $\Gamma_{(\varepsilon),|\xi|}$. Indeed, this symbol is holomorphic in $\lambda$ for $(\xi, \lambda)$ such that $|\xi|^{2}+|\lambda|>1$ and 
$|\xi|^{2}>c|\lambda|$ (where $c>0$ is the same as in (3.43) and (3.44)). Since $0<\rho<(2 c+1)^{-1}$, we have for $\lambda$ between $\Gamma_{(\varepsilon)}$ and $\Gamma_{(\varepsilon),|\xi|}$

$$
\begin{gathered}
\rho \leq|\lambda| \leq|\xi|^{2} \rho(1-\rho)^{-1}, \quad c|\lambda| \leq c|\xi|^{2} \rho(1-\rho)^{-1}<2^{-1}|\xi|^{2}<|\xi|^{2} \\
\text { and }|\xi|^{2}+|\lambda|>1 \quad \text { for } \quad(\xi, \lambda) \in I_{2} .
\end{gathered}
$$

The density $J_{2}^{s}(x)$ is represented as the sum $J_{2, \rho}^{s}(x)+\sum_{j=1,2} J_{2, j}^{s}(x)$, where $J_{2, j}^{s}$ and $J_{2, \rho}^{s}$ correspond to (3.49), with the interior integral replaced by the integral over $\Gamma_{j,|\xi|}^{\varepsilon}:=|\xi|^{2}(1-\rho)^{-1} \Gamma_{j, \varepsilon}$ and over $\Gamma_{\rho,|\xi|}^{\varepsilon}:=|\xi|^{2}(1-\rho)^{-1} \Gamma_{\rho}^{\varepsilon}$. The density $J_{1}^{s}(x)$ is equal to the sum $\sum_{j=1,2} J_{1, j}^{s}(x)$, where the interior integral in (3.49) for the term $J_{1, j}^{s}$ is over the contour $\Gamma_{j, \varepsilon} \backslash D_{1-|\xi|^{2}}\left(D_{r}:=\{\lambda,|\lambda|<r\}\right)$.

Set $\lambda:=\exp \left(i \varepsilon(-1)^{j+1}\right) t^{2}$ on $\Gamma_{j,|\xi|}^{\varepsilon}\left(\right.$ for $\left.|\xi|^{2}>1-\rho\right)$ and on $\Gamma_{j, \varepsilon} \backslash D_{1-|\xi|^{2}}$ (for $\left.|\xi|^{2} \leq 1-\rho\right)$, where $t>0$ is a new variable. Then we have

$$
\begin{aligned}
& \left(J_{1, j}^{s}+J_{2, j}^{s}\right)(x)= \\
& \quad=2(2 \pi)^{-(n+1)} i \exp \left((-1)^{j} i \varepsilon(s-1)\right) \sum_{l} \varphi_{l}(x) \int_{F} t^{-2 s+1} \operatorname{tr}\left(\sum_{0}^{m} a_{-2-k}\left(x, \xi, \lambda_{\varepsilon}(t)\right)\right) d t d \xi \\
& \quad(3.53)
\end{aligned}
$$

where $\lambda_{\varepsilon}(t):=\exp \left((-1)^{j+1} i \varepsilon\right) t^{2}$, Re $s>n / 2$, and $F$ is the domain

$$
\left\{(\xi, t):|\xi|^{2}+t^{2} \geq 1,|\xi|^{2} \leq 1-\rho, t \geq \rho^{\frac{1}{2}}\right\} \cup\left\{(\xi, t):|\xi|^{2} \geq 1-\rho, t \geq|\xi| \rho^{\frac{1}{2}} /(1-\rho)^{\frac{1}{2}}\right\} .
$$

Since $a_{-k}(x, \xi, \lambda)$ are nonsingular for $\lambda \in \Gamma_{j, \varepsilon}$ and since $r \cdot(\xi, t) \in F$ for $(\xi, t) \in F$ and $r \geq 1$, we see that (3.53) can be written as follows:

$$
\begin{aligned}
\left(J_{1, j}^{s}+\right. & \left.J_{2, j}^{s}\right)(x)=2(2 \pi)^{-(n+1)} i \exp \left((-1)^{j} i \varepsilon(s-1)\right) \times \\
& \times \sum_{l} \sum_{k=0}^{m}(2 s+k-n)^{-1} \varphi_{l}(x) \int_{F_{1}} t^{-2 s+1} \operatorname{tr} a_{-2-k}\left(x, \xi, \lambda_{\varepsilon}(t)\right) d t \omega_{n+1}
\end{aligned}
$$

where $F_{1}=F \cap\left\{(\xi, t): \xi^{2}+t^{2}=1\right\}$ and $\omega_{n+1}$ is the volume form on the unit sphere in $\mathbb{R}_{\xi, t}^{n+1}$. The integral over the compact $F_{1}$ in (3.54) is an entire function of $s \in \mathbb{C}$. So the right side of (3.54) realizes the analytic continuation of the density $\left(J_{1, j}^{s}+J_{2, j}^{s}\right)(x)$ to a meromorphic in $s \in \mathbb{C}$ density with no more than simple poles at the points $s_{k}=(n-k) / 2,0 \leq k \leq m$.

Since $\lambda=\rho(1-\rho)^{-1}|\xi|^{2} \exp (i \varphi)$ on $\Gamma_{\rho,|\xi|}^{\varepsilon}$ (where $\varepsilon \geq \varphi \geq-\varepsilon$ ), we have

$$
J_{\rho}^{s}(x)=-i(2 \pi)^{-(n+1)} \sum_{l} \sum_{k=0}^{m} \varphi_{l}(x) \int I_{l, k}^{\varepsilon, \rho}(s, \xi) d \xi
$$


where the integral is over $\left\{\xi:|\xi|^{2} \geq 1-\rho\right\}$ and

$$
\begin{gathered}
I_{l, k}^{\varepsilon, \rho}(s, \xi):=\int_{-\varepsilon}^{\varepsilon} \exp (-i \varphi(s-1)) a_{-2-k}(x, \xi, \lambda(\varphi,|\xi|)) d \varphi|\xi|^{-2(s-1)} \rho_{+}^{-(s-1)} \\
\lambda(\varphi,|\xi|):=|\xi|^{2} \rho_{+} \exp (i \varphi), \quad \rho_{+}:=\rho(1-\rho)^{-1}
\end{gathered}
$$

The symbol (3.56) is positive homogeneous of degree $(-2 s-k)$ in $\xi$. It is analytic in $s \in \mathbb{C}$ and nonsingular. So (3.55) realizes a meromorphic continuation of $J_{\rho}^{s}(x)$ to the whole complex plane $\mathbb{C} \ni s$. Namely

$$
J_{\rho}^{s}(x)=2^{-1}(2 \pi)^{-(n+1)} \sum_{l} \sum_{k=0}^{m} \varphi_{l}(x)\left(\int_{|\xi|=1} I_{l, k}^{\varepsilon, \rho}(s, \xi)_{x} \omega_{n}\right)\left(s-s_{k}\right)^{-1}(1-\rho)^{-\left(s-s_{k}\right)}
$$

where $\omega_{n}$ is the volume form on the unit sphere in $\mathbb{R}^{n}$. The formulas (3.54) and (3.57) provide us with a meromorphic continuation of the density $P_{i n t}^{s, \varepsilon}(x)$. Together with the estimate (3.51) they provide us with the estimate (3.48). (However, with the sum in it over all $s_{k}, 0 \leq k \leq m$.) The analytic continuation of the density defined by the sum of the integrals (3.49) with the interior integral over the contour $\Gamma_{(\pi)}$ (i.e., with $\varepsilon=\pi$ ) is regular in $s=s_{k}$ for $\left(-s_{k}\right) \in \mathbb{Z}_{+} \cup 0$. (This assertion is obtained in the proof of Theorem 3.1.) For $|\xi|^{2}>1-\rho$ the interior integral over $\Gamma_{(\varepsilon)}$ in $(3.49)$ is equal to the integral over $\Gamma_{(\pi)}$. So the estimate (3.48) is satisfied, where the sum over $s_{k}, 0 \leq k \leq m$, such that $\left(-s_{k}\right) \notin \mathbb{Z}_{+} \cup 0$.

Let Re $s>n / 2$ and let $p_{j, \partial}^{s, \varepsilon}(x)$ be the density on $M_{j}$ corresponding to the term in (3.47) determined by the $\nu$-transmission interior boundary conditions. ${ }^{73}$ It is defined by the restrictions to the diagonal $\bar{M}_{j} \hookrightarrow \bar{M}_{j} \times \bar{M}_{j}$ of the kernel for the operator

$$
\frac{i}{2 \pi} \int_{\Gamma_{(\varepsilon)}} \lambda^{-s} d \lambda p_{j} \sum_{l} \psi_{l} \mathcal{D}_{m, U_{l}} \varphi_{l}
$$

where $\mathcal{D}_{m}=\mathcal{D}_{m}(\lambda, \nu)$ is defined by (3.9), (3.10), and (3.12).

The operator $\mathcal{D}_{m}$ is defined by the symbols $\sum d_{-2-k}, 0 \leq k \leq m$, in a coordinate chart $\left(x^{\prime}, t\right), U_{i} \subset \mathbb{R}^{n-1} \times \mathbb{R}^{1}$ (where $N \cap U_{i}=\left(\mathbb{R}^{n-1} \times 0\right) \cap U_{i}$ and the structure $\mathbb{R}^{n-1} \times \mathbb{R}^{1}$ corresponds to the direct product structure of the metric $g_{M}$ near $\left.N\right)$. Its action on $f, f \in D R_{c}^{\bullet}\left(\mathbb{R}^{n-1} \times\left(\mathbb{R}_{+} \backslash 0\right)\right)$, can be represented for $t>0, t_{1}>0$, as

\footnotetext{
${ }^{73}$ From now on we'll suppose that $\partial M=\emptyset$. Estimates of the contributions into $\zeta_{\nu, \bullet}\left(s ; p_{j}\right)$ from the Dirichlet and the Neumann boundary conditions on the components of $\partial M_{j} \backslash N$ are analogous to the estimates for the contributions from the $\nu$-transmission interior boundary conditions.
} 
follows ([Se1], (26)-(28)):

$$
\begin{aligned}
\sum_{k=0}^{m} \operatorname{Op}\left(\theta_{1} d_{-2-k}\right) f\left(x^{\prime}, t\right) & :=\sum_{0}^{m}(2 \pi)^{-n} \iint \exp \left(i\left(\xi^{\prime}, x^{\prime}\right)\right) \theta_{1} d_{-2-k}^{-} f^{-}\left(\xi^{\prime}, t_{1}\right) d t_{1} d \xi^{\prime}, \\
d_{-2-k}^{-}\left(x^{\prime}, t, \xi^{\prime}, t_{1}, \lambda\right) & :=-\int_{\Gamma_{-}} \exp \left(-i \tau t_{1}\right) d_{-2-k}\left(x^{\prime}, t, \xi^{\prime}, \tau, \lambda\right), \\
f^{-}\left(\xi^{\prime}, t_{1}\right) & =\int \exp \left(-i\left(\xi^{\prime}, y\right)\right) f\left(y, t_{1}\right) d y,
\end{aligned}
$$

where $\Gamma_{-}=\Gamma_{-}\left(\xi^{\prime}, \lambda\right)$ is a simple contour in the half-plane $\operatorname{Im} \tau<0$ which once goes round (in the direction opposite to the clockwise) the only zero of the principal symbol $\left(b_{2}\left(x^{\prime}, \xi^{\prime}, \tau\right)-\lambda\right)$ id of the Laplacian $\left(\Delta^{\bullet}-\lambda\right.$ id $) .{ }^{74}$ Lemmas 2 and 3 of [Se2] and Lemma 2 of [Se1] claim that the integral over $\mathbb{R}_{+}$(where $\left.U_{i} \cap\left(\mathbb{R}^{n-1} \times \mathbb{R}_{+}\right)=U_{i} \cap M_{j}\right)$ $\int d_{-2-k}^{-}\left(x^{\prime}, t, \xi^{\prime}, t, \lambda\right) d t$ is a symbol of $\left(x^{\prime}, \xi^{\prime}, \lambda\right)$ positive homogeneous of degree $(-2-k)$ in $\left(\xi^{\prime}, \lambda^{1 / 2}\right)$. It claims also that the kernel on $\mathbb{R}^{n-1}$ defined by the integral over $[T, \infty) \subset \mathbb{R}_{+}($for an arbitrary $T>0$ )

$$
\int \exp \left(i\left(\xi^{\prime}, x^{\prime}-y^{\prime}\right)\right) d \xi^{\prime} \int_{\Gamma} d \lambda \int_{T}^{\infty} d t \theta_{1}\left(\xi^{\prime}, \lambda\right) \lambda^{-s} d_{-2-k}\left(x^{\prime}, t, \xi^{\prime}, t, \lambda\right)
$$

is an entire function of $s \in \mathbb{C}$, smooth in $x^{\prime}, y^{\prime}, s$ and vanishing at $s$ for $(-s) \in \mathbb{Z}_{+} \cup 0$. The latter assertion is an immediate consequence of the estimate ([Se1], (29)) for $d_{-2-k}^{-}$:

$$
\begin{aligned}
& \left|D_{x^{\prime}}^{\gamma} D_{\xi^{\prime}}^{q} t^{r} t_{1}^{l} D_{t}^{m} D_{t_{1}}^{n} D_{\lambda}^{p} d_{-2-k}^{-}\left(x^{\prime}, t, \xi^{\prime}, t_{1}, \lambda\right)\right| \leq \\
& C_{1} \exp \left(-c_{\varepsilon}\left(|t|+\left|t_{1}\right|\right)\left(|\xi|+|\lambda|^{1 / 2}\right)\right)\left(1+|\xi|+|\lambda|^{1 / 2}\right)^{-1-k-(|q|+2 p)-(r+l)+m+n}
\end{aligned}
$$

with positive constants $C_{1}(\varepsilon)$ and $c_{\varepsilon}$ independent of $\nu \in \mathbb{R}^{2} \backslash(0,0)$.

Proposition 3.7. The analytic continuation to the whole complex plane $\mathbb{C} \ni s$ of the integral over $M_{j}$ of $p_{j, \partial}^{s, \varepsilon}(x)$ (which is defined for $\operatorname{Re} s>n / 2$ ) is estimated by

$$
\begin{aligned}
& \left|\int_{M_{j}} p_{j, \partial}^{s, \varepsilon}(x)\right|<c_{1, \varepsilon} \max \left(\rho^{-\operatorname{Re} s}, 1\right) \exp (\varepsilon|\operatorname{Im} s|) \sum\left|s-s_{k}\right|^{-1}+ \\
+ & c_{2, \varepsilon} \exp (\varepsilon|\operatorname{Im} s|)\left(2 c_{\varepsilon}^{2(\operatorname{Re} s-1)} \Gamma\left(2(1-\operatorname{Re} s), c_{\varepsilon} \rho^{1 / 2}\right)+\pi \rho \max \left(\rho^{-\operatorname{Re} s}, 1\right)\right),
\end{aligned}
$$

where the sum is over $1 \leq k \leq m+1$ such that $\left(-s_{k}\right) \notin \mathbb{Z}_{+} \cup 0$.

Proof. The trace of the operator (3.58) for Re $s>n / 2$ is given by the integral

$$
(2 \pi)^{-n} \sum_{l} \int d x^{\prime} d t \varphi_{l}\left(x^{\prime}, t\right) \int d \xi^{\prime}\left(\frac{i}{2 \pi} \int_{\Gamma_{(\varepsilon)}} \lambda^{-s} d \lambda \theta_{1}\left(\xi^{\prime}, \lambda\right) \sum_{k=0}^{m} d_{-2-k}^{-}\left(x^{\prime}, t, \xi^{\prime}, t, \lambda\right)\right)
$$

${ }^{74}$ The whole symbol of $\Delta^{\bullet}$ does not depend on $t$ in the neighborhood of $N$. 
(We suppose that $\varphi_{l}\left(x^{\prime}, t\right)$ are independent of $t$ for $0 \leq|t| \leq 1$.) It follows from the estimate (3.59) that the density on $N$ corresponding to the integral

$$
\int_{\mathbb{R}_{+}} d t\left(\varphi_{l}\left(x^{\prime}, t\right)-\varphi_{l}\left(x^{\prime}, 0\right)\right) \int d \xi^{\prime} \operatorname{tr}\left(\frac{i}{2 \pi} \int_{\Gamma_{(\varepsilon)}} \lambda^{-s} d \lambda \theta_{1} \sum d_{-2-k}^{-}\right)
$$

has the analytic continuation which is an entire function of $s \in \mathbb{C}$ and which satisfies the estimate

$$
\begin{gathered}
C_{\varepsilon, m, n_{1}} \exp (\varepsilon|\operatorname{Im} s|) h_{\rho}\left(c_{\varepsilon}, \operatorname{Re} s\right) \int(1+|\xi|)^{-n_{1}} \exp \left(-c_{\varepsilon}\left|\xi^{\prime}\right|\right) d \xi^{\prime}, \\
h_{\rho}\left(c_{\varepsilon}, \operatorname{Re} s\right):=\int_{\rho}^{\infty} \exp \left(-c_{\varepsilon} t^{1 / 2}\right) t^{-\operatorname{Re} s} d t+\pi \rho \max \left(\rho^{-\operatorname{Re} s}, 1\right)= \\
=2 c_{\varepsilon}^{2(\operatorname{Re} s-1)} \Gamma\left(2(1-\operatorname{Re} s), c_{\varepsilon} \rho^{1 / 2}\right)+\pi \rho \max \left(\rho^{-\operatorname{Re} s}, 1\right),
\end{gathered}
$$

where $n_{1} \in \mathbb{Z}_{+}$is sufficiently large.

The density on $N$ is defined by the integral

$$
p_{l, j, \partial}^{s, \varepsilon}\left(x^{\prime}\right):=(2 \pi)^{-n} \varphi_{l}\left(x^{\prime}, 0\right) \int_{\mathbb{R}_{+}} d t \int d \xi^{\prime} \operatorname{tr}\left(\frac{i}{2 \pi} \int_{\Gamma_{(\varepsilon)}} \lambda^{-s} d \lambda \theta_{1} \sum_{k=0}^{m} d_{-2-k}^{-}\right)
$$

which is absolutely convergent for $\operatorname{Re} s>(n-1) / 2$. Hence it is analytic in $s$ for such $s$. The integral over $t \in \mathbb{R}_{+}$of $d_{-2-k}^{-}$is a positive homogeneous of degree $(-2-k)$ in $\left(\xi^{\prime}, \lambda^{1 / 2}\right)$ symbol, which is smooth in $\left(x^{\prime}, \xi^{\prime}, \lambda\right)$ and analytic in $\lambda$ for $c|\lambda|<\left|\xi^{\prime}\right|^{2}$ (where $c$ is the same as in (3.43), and (3.44)) and in $\lambda \in \Lambda_{\varepsilon / 2}:=\{\lambda: \varepsilon / 2<\arg \lambda<2 \pi-\varepsilon / 2\}$ for $\left(\xi^{\prime}, \lambda\right) \neq(0,0)$ ([Se1], Lemma 2, [Se2], Lemma 2). So the proof of Proposition 3.6 is valid also for the density $(3.62)$ (where $(x, \xi, n)$ are replaced by $\left.\left(x^{\prime}, \xi^{\prime}, n-1\right)\right)$. We conclude that this density has a meromorphic continuation $p_{l, j, \partial}^{s, \varepsilon}\left(x^{\prime}\right)$ with no more than simple poles at the points $s_{1}, \ldots, s_{m+1}$. This proof provides us with the estimate

$$
\left|p_{l, j, \partial}^{s, \varepsilon}\left(x^{\prime}\right)\right|<c_{1, \varepsilon} \max \left(\rho^{-\operatorname{Re} s}, 1\right) \exp (\varepsilon|\operatorname{Im} s|) \sum\left|s-s_{k}\right|^{-1},
$$

where the sum is over $1 \leq k \leq m+1$. The analytic continuation of the density on $N$, defined by the sum (over $l$ ) of the integrals (3.62) for the interior integrals over the contour $\Gamma_{(\pi)}$ (i.e., with $\varepsilon=\pi$ ) is regular at $s=s_{k}$ for $\left(-s_{k}\right) \in \mathbb{Z}_{+} \cup 0$. (It is proved in Theorem 3.1.) For $\left|\xi^{\prime}\right|^{2}>1-\rho$ the integral over $\Gamma_{(\varepsilon)}$ in (3.62) is equal to the integral over $\Gamma_{(\pi)}$. Hence the estimate (3.63) is satisfied if the sum is over $k, 1 \leq k \leq m+1$, such that $\left(-s_{k}\right) \notin \mathbb{Z}_{+} \cup 0$. The estimate (3.60) follows from (3.63), (3.61).

The estimate (3.40) follows from Propositions 3.6, 3.7, and from (3.46). Thus Proposition 3.4 is proved.

Proof of Proposition 3.5. The estimate (3.46) in the proof of Proposition 3.4 is satisfied by the integral of $\left(r_{\lambda}^{m}\right)_{x_{1}, x_{2}}$. So it is enough to obtain the estimate (3.41) for 
the kernel of

$$
\frac{i}{2 \pi} \int_{\Gamma_{(\varepsilon)}} \lambda^{-s} P_{\lambda}^{m} d \lambda
$$

where $\operatorname{Re} s>n / 2$. The term $p_{\text {int }}^{s, \varepsilon}\left(x_{1}, x_{2}\right)$ in this kernel has the same form as in (3.49) but with the addition factor $\exp \left(i \xi\left(x_{1}-x_{2}\right)\right)$ under the integral sign (where $x$ and $\varphi_{j}(x)$ are replaced by $x_{1}$ and by $\left.\psi_{j}\left(x_{1}\right) \varphi_{j}\left(x_{2}\right)\right)$. The integration over the domains (3.50) in the integral corresponding to (3.49) represents this kernel as the $\operatorname{sum}\left(J_{0, \varepsilon}^{s}+J_{1, \varepsilon}^{s}+J_{2, \varepsilon}^{s}\right)\left(x_{1}, x_{2}\right)$, where $J_{l, \varepsilon}^{s}$ corresponds to the integration over the appropriate domain in (3.50). The term $J_{0, \varepsilon}^{s}\left(x_{1}, x_{2}\right)$ satisfies the estimate (3.51) if $\operatorname{Re} s>n / 2$. (In this estimate $\max \left(\rho^{-\operatorname{Re} s}, 1\right)$ can be replaced by $\rho^{-\operatorname{Re} s}$ since Re $s>0$ and since $0<\rho<1$.)

The contour $\Gamma_{(\varepsilon)}$ of the interior integral in (3.49) for $J_{2, \varepsilon}^{s}$ can be replaced by the contour $\Gamma_{(\varepsilon),|\xi|}$ defined by (3.52). The sum of the integrals over the straight line pieces of $\Gamma_{(\varepsilon)}$ and of $\Gamma_{(\varepsilon),|\xi|}$ in the kernel $\left(J_{1, \varepsilon}^{s}+J_{2, \varepsilon}^{s}\right)\left(x_{1}, x_{2}\right)$ has the same form as (3.53) (but with the factor $\exp \left(i\left(x_{1}-x_{2}\right) \xi\right)$ under the integral sign). The integral over the circle part of $\Gamma_{(\varepsilon),|\xi|}$ for the kernel $J_{2, \varepsilon}^{s}\left(x_{1}, x_{2}\right)$ is also completely analogous to (3.55). This provides us with the estimate for the kernel $p_{i n t}^{s, \varepsilon}\left(x_{1}, x_{2}\right)($ where $\operatorname{Re} s>n / 2)$ :

$$
\left|p_{\text {int }}^{s, \varepsilon}\left(x_{1}, x_{2}\right)\right|<C_{\varepsilon} \exp (\varepsilon|\operatorname{Im} s|) \rho^{-\operatorname{Re} s}(\operatorname{Re} s-n / 2)^{-1} .
$$

The proof of (3.41) for an arbitrary closed manifold $M$ follows from the estimate (3.64) together with the estimate (3.46) for $\left(r_{\lambda}^{m}\right)_{x_{1}, x_{2}}$. (They also give us the proof of (3.41) for a part $p_{i n t}^{s, \varepsilon}\left(x_{1}, x_{2}\right)$ of the kernel $\left(T_{-s}\right)_{x_{1}, x_{2}}$ defined by a local parametrix $\left.\sum_{j} \psi_{j} P_{\lambda, i n t}^{m} \varphi_{j}.\right)$ If $\left(M, g_{M}\right)$ is mirror symmetric with respect to $\left(N, g_{N}\right)$ (and the $\nu$ transmission interior boundary conditions are given on $N)$ then the kernel $\left(T_{-s}\right)_{x_{1}, x_{2}}$ for $\operatorname{Re} s>-n / 2$ can be represented by the formulas (analogous to (2.54), (2.55), and to $(2.118))$, where $\nu=(\alpha, \beta) \neq(0,0)$ and $T_{-s}^{M}$ corresponds to a closed manifold $\left(M, g_{M}\right)$ (or to $\nu_{0}=(1,1)$ that is the equivalent according to Proposition 1.1):

$$
\begin{array}{ll}
\left(T_{-s}\right)_{x_{1}, x_{2}}=\left(T_{-s}^{M}\right)_{x_{1}, x_{2}}+\frac{\beta^{2}-\alpha^{2}}{\alpha^{2}+\beta^{2}}\left(\sigma_{1}^{*} T_{-s}^{M}\right)_{x_{1}, x_{2}} \quad \text { for } x_{1}, x_{2} \in M_{1}, \\
\left(T_{-s}\right)_{x_{1}, x_{2}}=\left(T_{-s}^{M}\right)_{x_{1}, x_{2}}+\frac{\alpha^{2}-\beta^{2}}{\alpha^{2}+\beta^{2}}\left(\sigma_{1}^{*} T_{-s}^{M}\right)_{x_{1}, x_{2}} \quad \text { for } x_{1}, x_{2} \in M_{2}, \\
\left(T_{-s}\right)_{x_{1}, x_{2}}=\frac{2 \alpha \beta}{\alpha^{2}+\beta^{2}}\left(T_{-s}^{M}\right)_{x_{1}, x_{2}} \quad \text { for } x_{1}, x_{2} \text { from different } M_{k},
\end{array}
$$

where $\sigma_{1}$ is the mirror symmetry on $M$ with respect to $N$, acting on the variable $x_{1}$. The kernel $\left(T_{-s}^{M}\right)_{x_{1}, x_{2}}$ can be analytically (meromorphically) continued to the whole complex plane $\mathbb{C} \ni s$ (separately on the diagonal $x_{1}=x_{2}$ and off the diagonal). It follows from [Se2], Theorem 1 or from the proof of Theorem 3.1. Hence (3.65) is true 
for all $s \in \mathbb{C}$. So the estimate (3.41) is satisfied also in the case of the $\nu$-transmission interior boundary conditions on $N$ if $\left(M, g_{M}\right)$ is mirror-symmetric with respect to $N$.

The boundary term ${ }^{75} \sum \psi_{j} \mathcal{D}_{m, U} \varphi_{j}$ of the parametrix $P^{m}$ can be identified with the same term in the mirror-symmetric case (as it is defined in a neighborhood $N \times I$ of $N=N \times 0, I=[-2,2])$. The estimate (3.46) for the integral over $\Gamma_{(\varepsilon)}$ of $\left(r_{\lambda}^{m}\right)_{x_{1}, x_{2}}$ is satisfied for the mirror-symmetric case also. So the estimate (3.41) is satisfied by the kernel $p_{x_{1}, x_{2}}^{\partial, \varepsilon}(s)$ of the operator $\sum \psi_{j} \mathcal{D}_{m, U} \varphi_{j}$. This estimate for $p_{x_{1}, x_{2}}^{\partial, \varepsilon}(s)$ together with the estimates $(3.64),(3.46)$ for $p_{i n t}^{s, \varepsilon}\left(x_{1}, x_{2}\right)$, and with the estimate (3.46) of the integral of $\left(r_{\lambda}^{m}\right)_{x_{1}, x_{2}}$ over $\Gamma_{(\varepsilon)}$ provides us with the estimate (3.41).

3.5. Appendix. Trace class operators and their traces. A bounded linear operator $A$ acting in a separable Hilbert space $H$ is a trace class operator if the series of its singular numbers (i.e., of the arithmetic square roots of the eigenvalues for the self-adjoint operator $A^{*} A$ ) is absolutely convergent. If $A$ is a trace class operator then its matrix trace exists for any orthonormal basis $\left(e_{i}\right)$ in $H$ :

$$
\sum\left(A e_{i}, e_{i}\right)=: \operatorname{Sp} A
$$

and this sum is independent of the orthonormal basis $([\mathrm{Kr}])$. It is called the matrix trace of $A$. The Lidskii theorem ([Li]) claims that if $A$ is a trace class operator then the series of its eigenvalues is absolutely convergent: $\sum\left|\lambda_{j}(A)\right|<\infty$ and its trace $\operatorname{Tr} A:=\sum \lambda_{j}(A)$ is equal to its matrix trace: $\operatorname{Tr} A=\operatorname{Sp} A$. (Here the sums are over all the eigenvalues $\lambda_{j}(A)$ of $A$ including their algebraic multiplicities, [Ka], Ch. 1 , $\S 5.4$.

Proposition 3.8. For $t>0$ the operators $\exp \left(-t \Delta_{\nu_{0}, j}\right)$ and $p_{1} \exp \left(-t \Delta_{\nu_{0}, j}\right)$ are trace class operators in the $L_{2}$-completion $\left(D R^{j}(M)\right)_{2}$ of $D R^{j}(M)^{76}$ and their traces are equal to the integrals of the densities defined by the restrictions to the diagonals of their kernels:

$$
\begin{aligned}
\operatorname{Tr} \exp \left(-t \Delta_{\nu_{0}, j}\right) & =\sum_{r=1,2} \int_{\bar{M}_{r}} \operatorname{tr}\left(*_{x_{2}} i_{M_{r}}^{*} E_{t, x_{1}, x_{2}}^{j}\left(\nu_{0}\right)\right), \\
\operatorname{Tr}\left(p_{1} \exp \left(-t \Delta_{\nu_{0}, j}\right)\right) & =\int_{\bar{M}_{1}} \operatorname{tr}\left(*_{x_{2}} i_{M_{1}}^{*} E_{t, x_{1}, x_{2}}^{j}\left(\nu_{0}\right)\right) .
\end{aligned}
$$

Here $p_{k}:\left(D R^{j}(M)\right)_{2} \rightarrow\left(D R^{j}\left(M_{k}\right)\right)_{2} \hookrightarrow\left(D R^{j}(M)\right)_{2}$ is the composition of the restriction to $M_{k}$ of differential forms and of their prolongation to $M$ by zero on another piece of $M, i_{M_{r}}: \bar{M}_{r} \hookrightarrow \bar{M}_{r} \times \bar{M}_{r}$ is an immersion of the diagonal, and the exterior product of the double forms (restricted to the diagonal) is implied.

\footnotetext{
${ }^{75}$ The operator $\mathcal{D}_{m}=\mathcal{D}_{m, \lambda}(\nu)$ is defined in the coordinate chart $\mathbb{R}^{n-1} \times \mathbb{R}^{1} \ni\left(x^{\prime}, t\right)$ by (3.12), (3.11), (3.10).

${ }^{76} D R^{j}\left(\bar{M}_{1}\right) \oplus D R^{j}\left(\bar{M}_{2}\right) \subset\left(D R^{j}(M)\right)_{2}$
} 
Proof. The operator $A_{t}:=\exp \left(-t \Delta_{\nu_{0}, j}\right)$ is positive definite on $\left(D R^{j}(M)\right)_{2}$ and for an arbitrary $f \in\left(D R^{j}(M)\right)_{2}, f \neq 0$, it holds $\left(A_{t} f, f\right)>0$ (where the scalar product on $\left(D R^{j}(M)\right)_{2}$ corresponds to $\left.(1.23)\right)$.

The operator $B_{t}:=p_{1} \exp \left(-t \Delta_{\nu_{0}, j}\right)$ is positive definite on the subspace $\left(D R^{j}\left(M_{1}\right)\right)_{2}$ of $\left(D R^{j}(M)\right)_{2}$. Namely $\left(B_{t} m, m\right)>0$ for $m \in\left(D R^{j}\left(M_{1}\right)\right)_{2}, m \neq 0$, and it is a nonnegative operator on $\left(D R^{\bullet}(M)\right)_{2}:\left(B_{t} f, f\right) \geq 0$ for $f \in\left(D R^{j}(M)\right)_{2}$.

The operator $\exp \left(-t \Delta_{\nu_{0}, j}\right)$ is self-adjoint on $\left(D R^{j}(M)\right)_{2}$ by Theorem 3.2. Its kernel $A\left(x_{1}, x_{2}\right)$ is smooth on $\bar{M}_{r_{1}} \times \bar{M}_{r_{2}}$ (as it is proved in Proposition 3.2) and its trace is equal to

$$
\operatorname{Tr} \exp \left(-t \Delta_{\nu_{0}, j}\right)=\sum_{k=1,2} \operatorname{Tr}\left(p_{k} \exp \left(-t \Delta_{\nu_{0}, j}\right)\right)=\sum_{k=1,2} \operatorname{Tr}\left(p_{k} \exp \left(-t \Delta_{\nu_{0}, j}\right) p_{k}\right) .
$$

(The matrix trace for $\exp \left(-t \Delta_{\nu_{0}, j}\right)$ in $\left(D R^{j}(M)\right)_{2}=: H$ can be computed with the help of an orthonormal basis $\left(e_{i}(1)\right),\left(e_{i}(2)\right)$ in $H$, where $\left(e_{i}(k)\right)$ is an orthonormal basis in $\left.H_{k}:=\left(D R^{j}\left(M_{k}\right)\right)_{2}.\right)$

The operator $A_{k}:=p_{k} \exp \left(-t \Delta_{\nu_{0}, j}\right) p_{k}$ acting in $H=H_{1} \oplus H_{2}$ has a continuous kernel $A_{k}\left(x_{1}, x_{2}\right)=E_{t, x_{1}, x_{2}}^{j}\left(\nu_{0}\right)$ on $\bar{M}_{k} \times \bar{M}_{k}$ (and it has the zero kernel on $\bar{M}_{k_{1}} \times \bar{M}_{k_{2}}$ for $\left.k_{1} \neq k_{2}\right)$. The operator $A_{k}$ is a self-adjoint operator acting in the Hilbert space $H_{k}$ and it is positive definite, $\left(A_{k} f, f\right)>0$ for $f \in H_{k}, f \neq 0$. So according to the Mercer theorem [GG], IV.3, [RiN], $\S 98$, the Fourier series for the kernel of $A_{k}$ by the eigenforms of $A_{k}$

$$
A_{k}\left(x_{1}, x_{2}\right)=\sum \mu_{j} \omega_{j}\left(x_{1}\right) \otimes \omega_{j}\left(x_{2}\right)
$$

(where $\mu_{j}>0$ are the eigenvalues of $A_{k}$ ) converges absolutely and uniformly with respect to $\bar{M}_{k} \times \bar{M}_{k}$. Hence integrating this series over the diagonals in $\bar{M}_{k} \times \bar{M}_{k}$ (for $k=1,2$ ) we obtain the equality (3.66):

$$
\operatorname{Tr} \exp \left(-t \Delta_{\nu_{0}, j}\right)=\sum_{k=1,2} \operatorname{Tr} A_{k}=\sum_{k=1,2} \int_{\bar{M}_{k}} \operatorname{tr}\left(i_{M_{k}}^{*} A_{k}\left(x_{1}, x_{2}\right)\right) .
$$

The equality (3.67) is obtained similarly

$$
\operatorname{Tr}\left(p_{1} \exp \left(-t \Delta_{\nu_{0}, t}\right)\right)=\operatorname{Tr}\left(p_{1} \exp \left(-t \Delta_{\nu_{0}, t}\right) p_{1}\right)=\int_{\bar{M}_{1}} \operatorname{tr}\left(i_{M_{1}}^{*} A_{1}\left(x_{1}, x_{2}\right)\right) .
$$

The proposition is proved.

Proposition 3.9. For Re $s>n / 2$ the operator $T_{-s}$ defined by the integral $l^{77}$ (3.18) and the operators $p_{j}\left(\Delta_{\nu}^{\bullet}\right)^{-s}$ are trace class operators $(n:=\operatorname{dim} M)$. The traces of

${ }^{77}$ The operator $T_{-s}$ for such $s$ is defined on $\left(D R^{\bullet}(M)\right)_{2}$ and it is equal to the direct sum of the operator $\left(\Delta_{\nu}^{\bullet}\right)^{-s}$ on the orthogonal complement to $\operatorname{Ker}\left(\Delta_{\nu}^{\bullet}\right)$ and of the zero operator on $\operatorname{Ker}\left(\Delta_{\nu}^{\bullet}\right)$, by Theorem 3.1 . 
these operators for $\operatorname{Re} s>n / 2$ are equal to the integrals over the diagonals of the denisities, defined by the restrictions of their kernels to these diagonals.

$$
\begin{aligned}
\operatorname{Tr}\left(\left(\Delta_{\nu}^{\bullet}\right)^{-s}\right) & =\sum_{r=1,2} \int_{\bar{M}_{r}} \operatorname{tr}\left(*_{x_{2}} i_{M_{r}}^{*} T_{-s}\left(x_{1}, x_{2}\right)\right), \\
\operatorname{Tr}\left(p_{j}\left(\Delta_{\nu}^{\bullet}\right)^{-s}\right) & =\int_{\bar{M}_{j}} \operatorname{tr}\left(*_{x_{2}} i_{M_{j}}^{*} T_{-s}\left(x_{1}, x_{2}\right)\right) .
\end{aligned}
$$

Proof. The kernel $T_{-s}\left(x_{1}, x_{2}\right)$ for Re $s>n / 2$ is continuous on $\bar{M}_{j_{1}} \times \bar{M}_{j_{2}}$ (Theorem 3.1). The operator $T_{-s}$ for such $s$ is nonnegative, $\left(T_{-s} f, f\right) \geq 0$, and self-adjoint. It is a trace class operator (Theorem 3.1). For Re $s>n / 2$ the equality holds (analogous to (3.68):

$$
\operatorname{Tr} T_{-s}=\sum_{j=1,2} \operatorname{Tr}\left(p_{j} T_{-s} p_{j}\right)
$$

The operator $p_{j} T_{-s} p_{j}$ is self-adjoint in the Hilbert subspace $\left(D R^{\bullet}\left(M_{j}\right)\right)_{2}$ of $\left(D R^{\bullet}(M)\right)_{2}$ and its kernel coincides with the kernel $K_{-s, j}$ of $T_{-s}$ on $\bar{M}_{j} \times \bar{M}_{j}$. So for Re $s>n / 2$ its kernel is continuous and, according to the Mercer theorem, the series on $\bar{M}_{j} \times \bar{M}_{j}$ for $K_{-s, j}$ by the eigenforms of $p_{j} T_{-s} p_{j}$ (analogous to (3.69)) is absolutely and uniformly convergent on $\bar{M}_{j} \times \bar{M}_{j}$. Hence for such $s$ the integral over the diagonal of the density, defined by the restriction of the kernel $K_{-s, j}$, is equal to $\operatorname{Tr}\left(p_{j} T_{-s} p_{j}\right)$. Thus the equalities (3.71) and (3.70) are proved.

Acknowledgements. I express my gratitude to R. Bott, W. Müller, C. Taubes, M. Wodzicki, and S.-T. Yau for stimulating discussions. I am indebted to J. Bernstein, A. Beilinson, D. Kazhdan, and I.M. Singer for a series of conversations on the theme of this paper. I am very grateful to the Department of Mathematics of Harvard University and to the Max-Planck-Institut für Mathematik for their hospitality and for financial support, which made it possible for me to work in the excellent intellectual atmosphere of these mathematical centers. The results and the methods of this paper were reported in the seminars of R. Bott, C. Taubes, and S.-T. Yau in Harvard during the Fall of 1992, in Oberseminar Max-Planck-Institut für Mathematik in May of 1993, and to H. McKean and L. Nirenberg in the Courant Institute in December of 1992. This paper was written in the Max-Planck-Institut für Mathematik.

\section{REFERENCES}

[BW] Bar-Natan, D., Witten, E.: Perturbative expansion of Chern-Simons theory. Commun. Math. Phys. 141, 423-440 (1991)

[BGV] Berline, N., Getzler, E., Vergne, M.: Heat kernels and Dirac operators. Grundl. math. Wiss. 298. Berlin, Heidelberg, New York: Springer-Verlag 1992 
[BZ1] Bismut, J.-M., Zhang, W.: Métriques de Reidemeister et métriques de Ray-Singer sur le déterminant de la cohomologie d'un fibré plat: une extension d'un résultat de Cheeger et Müller. C.R. Acad. Sci. Paris Sér. I Math. 313, 775-782 (1991)

[BZ2] Bismut, J.-M., Zhang, W.: Reidemeister, Milnor and Ray-Singer metrics: an extension of a theorem of Cheeger and Müller. Asterisque 205 Soc. Math. France 1992

[BT] Bott, R., Tu, L.W.: Differential forms in algebraic topology. Grad. Texts Math. 82. New York, Heidelberg, Berlin : Springer-Verlag 1982

[Bo] Bourbaki, N.: Éléments de mathématique. Fonctions d'une variable réelle. Paris: Diffusion C.C.L.S. 1976

[Ch] Cheeger, J.: Analytic torsion and the heat equation. Ann. of Math. 109, 259-322 (1979)

[DP] Dodziuk, J., Patodi, V.K.: Riemannian structures and triangulations of manifolds. J. Indian Math. Soc. 40, 1-52 (1976)

[Fr] Franz, W.: Über die Torsion einer Überdeckrung. J. reine angew. Math. 173, 245-254 (1935)

[GG] Gohberg, I., Goldberg, S.: Basic operator theory. Boston, Basel, Stuttgart: Birkhäuser 1981

[Gr] Greiner, P.: An asymptotic expansion for the heat equation. Arch. Rat. Mech. Anal. 41, 163-218 (1971)

[Hö] Hörmander, L.: The analysis of linear partial differential operators III. Grundl. math. Wiss. 274. Berlin, Heidelberg, New York, Tokyo: Springer-Verlag 1985

[KS] Kashiwara, M., Schapira, P.: Sheaves on manifolds. Grundl. math. Wiss. 292 Berlin, Heidelberg, New York: Springer-Verlag 1990

[Kas] Kassel, C.: Le residue non commutatif (d'apres M. Wodzicki). Semin. Bourbaki, 41 eme ann. 1988-89, Exp. 708 (1989)

[Ka] Kato, T.: Perturbation theory for linear operators. Grundl. math. Wiss. 132 Berlin, Heidelberg, New York: Springer-Verlag 1980

[Kr] Krein, M.G.: On certain new studies in the perturbation theory for selfadjoint operators. M.G. Krein: Topics in differential and integral equations and operator theory, pp. 107-172 Operator Theory : Adv. and Appl. v.7. Basel, Boston, Stuttgart : Birkhäuser 1983

[KwS] Kwun, K.W., Szczarba, R.H.: Product and sum theorems for Whitehead torsion. Ann. of Math. 82, 183-190 (1965)

[Li] Lidskii, V.B.: Nonselfadjoint operators with a trace. Dokl. Akad. Nauk SSSR 125, 485-487 (1959)

[Mi] Milnor, J.: Whitehead torsion. Bull. Amer. Math. Soc. 72, 358-426 (1966)

[Mü1] Müller, W.: Analytic torsion and $R$-torsion of Riemannian manifolds. Adv. in Math. 28, 233-305 (1978)

[Mü2] Müller, W.: Analytic torsion and $R$-torsion for unimodular representations. Preprint MPIM MPI/91-50, 1-46 (1991)

[Ra] Ray, D.B.: Reidemeister torsion and the Laplacian on lense spaces. Adv. in Math. 4, 109-126 (1970)

[RS] Ray, D.B., Singer, I.M.: $R$-torsion and the Laplacian on Riemannian manifolds. Adv. in Math. 7, 145-210 (1971)

[Re1] Reidemeister, K.: Die Klassifikation der Linsenraüme. Abhendl. Math. Sem. Hamburg 11, 102-109 (1935)

[Re2] Reidemeister, K.: Überdeckungen von Komplexen. J. rein angew. Math. 173, 164-173 (1935)

[dR1] de Rham, G.: Sur l'analysis situs des variétés a $n$ dimensions. J. Math. Pures Appl. (9) 10, 115-200 (1931) 
[dR2] de Rham, G.: Sur les nouveaux invariants topologique de M. Reidemeister. Mat. Sbornik (New Ser.) 1, 737-743 (1936)

[dR3] de Rham, G.: Complexes a automorphismes et homeomorphie differentiable. Ann. Inst. Fourier 2, 51-67 (1950)

[dR4] de Rham, G.: Variétés différentiables. Paris: Hermann 1955

[dRMK] de Rham, G., Maumary, S., Kervaire, M.A.: Torsion et type simple d'homotopie. Lect. Notes Math. 48 Springer 1967

[RiN] Riesz, F., Sz.-Nagy, B.: Leçons d'analyse fonctionelle. Academiai Kiadó. Budapest 1952

[Sc] Schwarz, A.S.: The partition function of degenerate quadratic functional and Ray-Singer torsion. Lett. Math. Phys. 2, 247-252 (1978)

[Se1] Seeley, R.T.: The resolvent of an elliptic boundary problem. Amer. J. Math. 91, 889-920 (1969)

[Se2] Seeley, R.T.: Analytic extension of the trace associated with elliptic boundary problems. Amer. J. Math. 91, 963-983 (1969)

[Sh] Shubin, M.A.: Pseudodifferential operators and spectral theory. Berlin, Heidelberg, New York: Springer-Verlag 1987

[T] Turaev, V. G.: Reidemeister torsion in knot theory. Uspekhi Mat. Nauk 41:1, 97-147 (1986) (Russian Math. Surveys 41:1, 119-182 (1986))

[V1] Vishik, S.M.: Analytic torsion of the boundary value problems. Dokl. Akad. Nauk SSSR 295, 1293-1298 (1987) (Soviet Math. Dokl. 36, 174-179 (1988))

[V2] Vishik, S.M.: Analytic torsion of finite-dimensional local systems. Dokl. Akad. Nauk SSSR 300, 1295-1299 (1988) (Soviet Math. Dokl. 37, 803-807 (1988))

[Wh] Whitehead, J.H.C.: Simple homotopy types. Amer. J. Math. 72, 1-57 (1950)

[W] Whitney, H.: Geometric integration theory. Princeton, NJ: Princeton Univ. Press 1957

[WW] Whittaker, E.T., Watson, G.N.: A course of modern analysis. Fourth edition. Cambridge, Gr. Brit.: Cambridge Univ. Press 1969

[Wi1] Witten, E.: Quantum field theory and the Jones polynomial. Commun. Math. Phys. 121, 351-399 (1988)

[Wi2] Witten, E.: On quantum gauge theories in two dimensions. Commun. Math. Phys. 141, 153-209 (1991)

[Wo] Wodzicki, M.: Local invariants of spectral asymmetry. Invent. Math. 75, 143-178 (1984)

Max-Planck Institut für Mathematik, Gottfried-Claren-Strasse 26, 53225 Bonn, GERMAnY

E-mail address: senia@mpim-bonn.mpg.de 